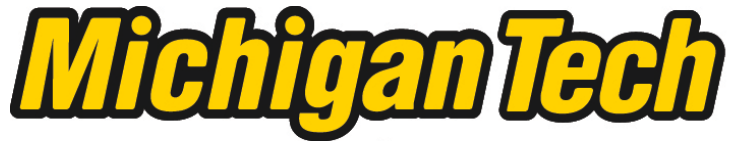 \\ Michigan Technological University Create the Future Digital Commons @ Michigan Tech
}

Dissertations, Master's Theses and Master's Reports - Open

Dissertations, Master's Theses and Master's

Reports

2005

\section{Price risk management in the copper market using commodity derivatives and options strategies}

Bold Sandagdorj

Michigan Technological University

Follow this and additional works at: https://digitalcommons.mtu.edu/etds

Part of the Agricultural and Resource Economics Commons

Copyright 2005 Bold Sandagdorj

\section{Recommended Citation}

Sandagdorj, Bold, "Price risk management in the copper market using commodity derivatives and options strategies", Master's Thesis, Michigan Technological University, 2005.

https://doi.org/10.37099/mtu.dc.etds/448

Follow this and additional works at: https://digitalcommons.mtu.edu/etds

Part of the Agricultural and Resource Economics Commons 


\title{
Price Risk Management in the Copper Market Using \\ Commodity Derivatives and Options Strategies
}

\author{
By \\ Bold Sandagdorj

\begin{abstract}
A THESIS
submitted in partial fulfillment of the requirements

for the degree of

MASTER OF SCIENCE IN MINERAL ECONOMICS
\end{abstract}

\section{MICHIGAN TECHNOLOGICAL UNIVERSITY}

2005

Copyright @ Bold Sandagdorj 2005 
This thesis, "Price Risk Management in the Copper Market Using Commodity Derivatives and Options Strategies", is hereby approved in partial fulfillment of the requirements for the Degree of MASTER OF SCIENCE IN MINERAL ECONOMICS.

School of Business and Economics

Signatures:

Thesis Advisor

Professor Mark C. Roberts, Ph. D

Department Chair

Professor Christa L. Walck, Ph. D

Date 


\section{TABLE OF CONTENTS}

LIST OF TABLES
LIST OF FIGURES
ACKNOWLEDGEMENTS

ABSTRACT

Page

i - iv

$\mathrm{v}-\mathrm{vii}$

viii - ix

$\mathrm{x}-\mathrm{xii}$

CHAPTER 1: INTRODUCTION

$1-5$

CHAPTER 2: DESCRIPTION OF RISK MANAGEMENT STRATEGIES

6 - 32

2.1. Background of Metal Exchanges

$6-7$

2.2. Spot versus Futures Markets

$8-11$

2.3. Description of Basic Derivatives

$11-21$

2.4. The Costs of Hedging

$21-24$

2.5. Risks and Rewards of Derivative Market Positions

$25-32$

CHAPTER 3: VALUING COMMODITY DERIVATIVES

33 - 56

3.1. Fair Values of Futures (Forward) Contracts

$33-36$

3.2. Valuing Asian Options

$37-42$

3.3. Data Description and Collection

$42-46$

3.4. Data Analysis

$46-56$

\section{CHAPTER 4: BACKGROUND OF ECONOMETRIC COMMODITY} MODELS AND LITERATURE REVIEW

$57-62$

CHAPTER 5: MODEL DEVELOPMENT FOR PRICE PREDICTION

$63-104$

5.1. Specification of the Model

$64-79$

5.2. Hypothesis Testing

80

5.3. Data Description and Collection

$81-82$

5.4. Two-Stage Least Squares Regression (2SLS) Analysis

$83-104$ 
CHAPTER 6: MONTE-CARLO SIMULATION ANALYSIS FOR THE LME COPPER PRICES

$105-111$

6.1. Short Term Forecasting of Copper Prices $105-107$

6.2. Monte-Carlo Simulation Analysis $108-111$

CHAPTER 7: EVALUATION OF RISK MANAGEMENT STRATEGIES

112 - 173

7.1. Options Strategies $112-169$

7.1.1. Bull Spreads $114-129$

7.1.2. Bear Spreads $130-138$

7.1.3. Butterfly Spreads $139-169$

7.2. Single Positions in Options $169-171$

7.2.1. A Producer's Strategies $169-170$

7.2.2. A Consumer's Strategies

7.3. Forward Contracts and Metal Swaps $172-173$

CHAPTER 8: CONCLUSIONS AND RECOMMENDATIONS

FOR FUTURE STUDIES 174 - 176

REFERENCES 177 - 180 APPENDICES 181 - 196 


\section{LIST OF TABLES}

\# Title $\quad$ Page

2.1. The LME Forward Prices (Calyon) ………..................................... 14

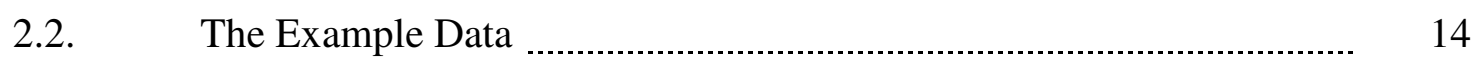

2.3. The Alumbrera's Price Fix Hedging Operation with the LME .......... 15

2.4. Basis Risk versus Outright Risk _................................................ 17

2.5. The Results of Metal Swaps Contract for 6 Months $\ldots$

2.6. A Summary Result of Price Fix Hedge on 17 March $2003 \ldots \ldots \ldots \ldots \ldots . . . . .22$

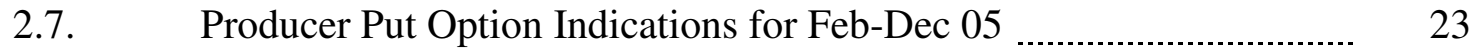

2.8. Monthly Average Prices (LME) _..................................................... 23

2.9. Payoff Matrix of an Offset Hedging _................................... 25

2.10. Variations in Gains or Losses Resulting from Differences in Cash and Future Price Movements …………………………........... 30

2.11. Summary of Risks and Rewards ................................................... 32

3.1. Cost of Carry Formula with Convenience Yields _.......................... 36

3.2. The LME Option Premiums (US\$/t) for Copper .............................. 43

3.3. The LME Option Premiums (US\$/t) for Copper .............................. 44

3.4. Valuations of Asian Options on 08.08 .02 (Implied Vol. 01.08.02) _.... 52

3.5. Valuations of Asian Options on 15.08 .02 (Implied Vol. 08.08.02) ..... 52

3.6. Valuations of Asian Options on 22.08 .02 (Implied Vol. 15.08.02) ..... 52

3.7. Valuations of Asian Options on 30.08.02 (Implied Vol. 22.08.02) _.... 53

3.8. Valuations of Asian Options on 05.09 .02 (Implied Vol. 30.08.02) ...... 53

3.9. Valuations of Asian Options on 22.02 .05 (Implied Vol. 21.02.05) _.... 53-54

3.10. The Summary of Options' Valuations …….................................... 56

5.1. Correlation Coefficients between Demand and its Exogenous Variables ………………………………......... 71

5.2. Correlation Coefficients between Supply and its Exogenous Variables _.............................................................. 77

5.3. The Expected Hypothesis ………………….................................. 80

5.4. The Units of Measurement for the Data ................................................ 82 
5.5. Correlations between Exogenous Variables

in the Structural Equation of Demand ……................................. 88

5.6. Correlations between Exogenous Variables

in the Structural Equation of Supply ............................................. 88

5.7. T-tests $\left(t_{r}\right)$ on the Structural Equation of Demand $\quad 89$

5.8. T-tests $\left(\mathrm{t}_{\mathrm{r}}\right)$ on the Structural Equation of Supply ............................ 89

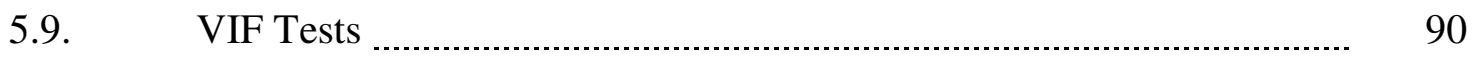

5.10. Critical d-Values _......................................................................... 94

6.1. The Results of the Simulation

6.2. Probability Distributions of Copper Price Forecasts _............................ 111

7.1. The Risk Free Interest Rates and Time to Maturities for Selected Options

7.2. Profit from a Bull Spread (Jan-06 Call options, X: 3100/3200) _......... 116

7.3. Profit from a Bull Spread (Jan-07 Call options, X: 3100/3200) _.......... 117

7.4. Profit from a Bull Spread (Jan-07 Call options, X: 3200/3300) $\ldots$

7.5. Profit from a Bull Spread (Jan-07 Call options, X: 3300/3400) .......... 118

7.6. The Payoffs of Bull Spreads Using Call Options for 2006 and 2007

$120-122$

7.7. Profit from a Bull Spread (Jan-06 Put options, X: 2200/2300) ............ 124

7.8. Profit from a Bull Spread (Dec-06 Put options, X: 2600/2700) …....... 125

7.9. Profit from a Bull Spread (Jan-07 Put options, X: 2300/2400) ............ 125

7.10. Profit from a Bull Spread (Dec-07 Put options, X: 2600/2700) …....... 125

7.11. Bull Spreads Using Put Options for 2006 and $2007 \ldots$ 127-129

7.12. Profit from a Bear Spread (Dec-06 Call options, X: 3400/3300) $\ldots . . . . . . \quad 131$

7.13. Profit from a Bear Spread (Dec-06 Call options, X: 3500/3400) _........ 132

7.14. Profit from a Bear Spread (Dec-06 Call options, X: 3600/3500) …..... 132

7.15. Profit from a Bear Spread (Jan-06 Call options, X: 3600/3500) …...... 133

7.16. Bear Spreads Using Call Options for 2006 and 2007 ........................ 135-137

7.17. Bear Spreads Using Put Options _.................................................. 138

7.18. Profit from a Butterfly Spread

(Jan-06 Call options, X: 3100/3200/3300) 
7.19. Profit from a Butterfly Spread

(Jan-06 Call options, X: 3200/3300/3400)

142

7.20. Profit from a Butterfly Spread

(Jan-06 Call options, X: 3300/3400/3500)

142

7.21. Profit from a Butterfly Spread

(Jan-06 Call options, X: 3400/3500/3600)

143

7.22. Profit from a Butterfly Spread

(Jan-07 Call options, X: 3100/3200/3300)

144

7.23. Profit from a Butterfly Spread

(Jan-07 Call options, X: 3200/3300/3400)

144

7.24. Profit from a Butterfly Spread

(Jan-07 Call options, X: 3300/3400/3500)

145

7.25. Profit from a Butterfly Spread

(Jan-07 Call options, X: 3400/3500/3600)

145

7.26. Butterfly Spreads Using Call Options for 2006 and 2007 (Case A) .... 147-149

7.27. Profit from a Butterfly Spread

(Dec-06 Call options, X: 3100/3200/3300)

7.28. Profit from a Butterfly Spread

(Dec-06 Call options, X: 3200/3300/3400)

7.29. Profit from a Butterfly Spread

(Dec-06 Call options, X: 3300/3400/3500)

152

7.30. Profit from a Butterfly Spread

(Dec-06 Call options, X: 3400/3500/3600)

152

7.31. Profit from a Butterfly Spread

(Dec-07 Call options, X: 3100/3200/3300)

154

7.32. Profit from a Butterfly Spread

(Dec-07 Call options, X: 3200/3300/3400)

154

7.33. Profit from a Butterfly Spread

(Dec-07 Call options, X: 3300/3400/3500)

155

7.34. Profit from a Butterfly Spread

(Dec-07 Call options, X: 3400/3500/3600)

155 
7.35. Butterfly Spreads Using Call Options for 2006 and 2007 (Case B) ..... 157-159

7.36. Profit from a Butterfly Spread

(Dec-06 Put options, X: 2300/2400/2500)

7.37. Profit from a Butterfly Spread

(Dec-06 Put options, X: 2400/2500/2600)

7.38. Profit from a Butterfly Spread

(Dec-06 Put options, X: 2500/2600/2700)

7.39. Profit from a Butterfly Spread

(Dec-07 Put options, X: 2300/2400/2500)

7.40. Profit from a Butterfly Spread

(Dec-07 Put options, X: 2400/2500/2600)

162

7.41. Profit from a Butterfly Spread

(Dec-07 Put options, X: 2500/2600/2700)

163

7.42. Butterfly Spreads Using Put Options for 2006 and 2007 $165-167$

7.43. Profit from a Strangle (Jan-07 Call/Put options, X: 3600/2700)

168

7.44. Probabilities for Written Call Options to be Profitable

170

7.45. Probabilities for Written Put Options to be Profitable

171

7.46. Evaluation of Risk Management Strategies in 2006-2007

173

\section{LIST OF DIAGRAMS}

2.1 Copper Production Processes ……............................................. 8

2.2. Solvent Extraction-Electrowinning (SX-EW) Process _....................... 9

2.3. The Matrix of Outcomes for an Offset Hedging .............................. 26

2.4. The Matrix of Outcomes for a Price Fix Hedging ….......................... 27 


\section{LIST OF FIGURES}

\# Title $\quad$ Page

1.1. Monthly Trading Volumes of the

LME Futures Contracts on Copper ……………………................ 4

1.2. Monthly Trading Volumes of the

LME Options Contracts on Copper ……………………………........ 4

2.1. The Relationship between Contango/Backwardation

Markets and Inventories _................................................................. 17

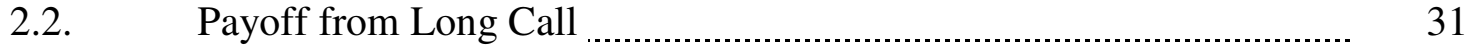

2.3. Payoff from Long Put _..................................................... 31

2.4. Payoff from Short Call _.......................................................... 31

2.5. Payoff from Short Put ........................................................... 31

3.1. Implied Vol. (Call, 01/08/02) _............................................ 47

3.2. Implied Vol. (Call, 08/08/02) ............................................................ 47

3.3. Implied Vol. (Call, 15/08/02) …………...................................... 47

3.4. Implied Vol. (Call, 22/08/02) ……............................................. 47

3.5. Implied Vol. (Call, 30/08/02) …………............................. 47

3.6. Implied Volatilities (Call, 21/02/05) _............................................. 48

3.7. Implied Volatilities (Put, 21/02/05) _............................................. 48

3.8. Volatility Smile _....................................................... 49

5.1. The Cobweb Model _.................................................................... 67

5.2. Supply of Funds ................................................................................ 75

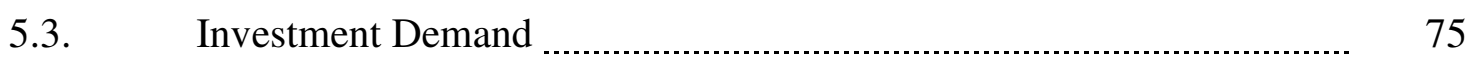

5.4. World $\mathrm{Cu}$ Demand \& LME Cash Prices _....................................... 78

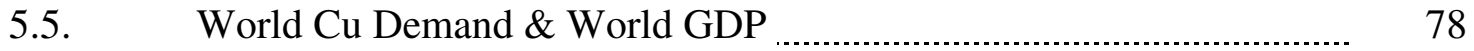

5.6. World $\mathrm{Cu}$ Demand \& Global IP Growth ……................................... 78

5.7. World $\mathrm{Cu}$ Supply \& LME Cash Prices $\ldots \ldots \ldots$

5.8. World Cu Supply \& Time (Technology) _......................................... 78

5.9. World $\mathrm{Cu}$ Supply \& Price of Oil _............................................... 78

5.10. World Cu Supply \& USD Index …………......................................... 
5.11. World $\mathrm{Cu}$ Supply \& LIBOR (1Y) Rate _...................................... 79

5.12. Distribution of the Residuals (Demand) …….................................... 93

5.13. Distribution of the Residuals (Supply) ……..................................... 93

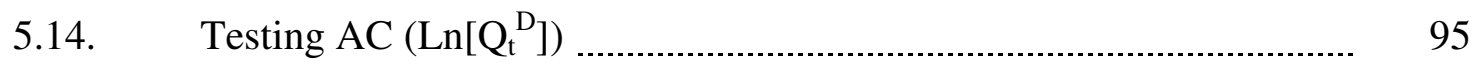

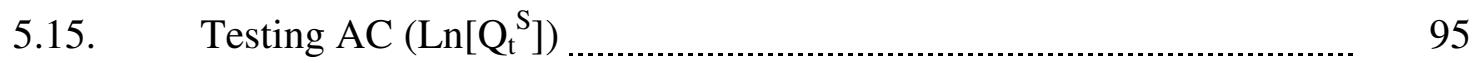

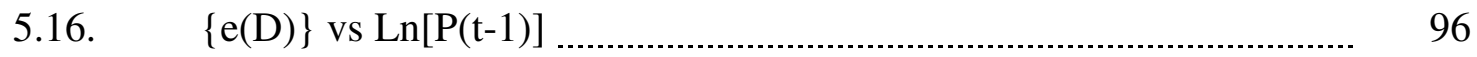

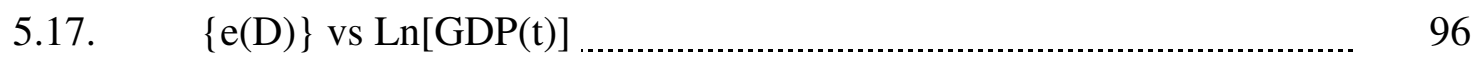

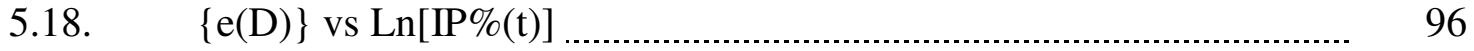

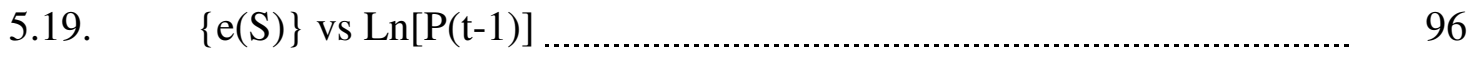

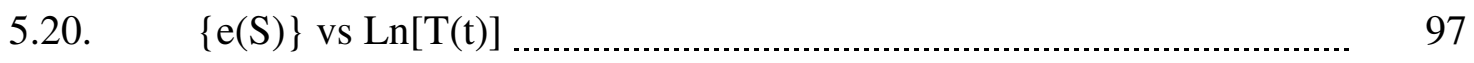

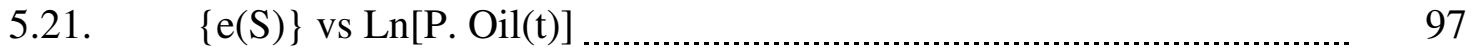

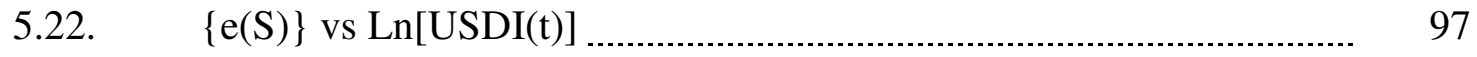

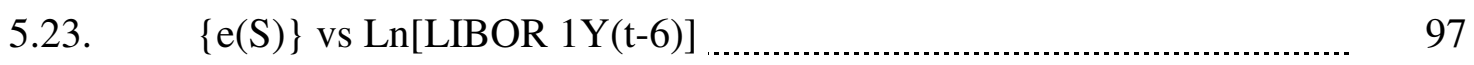

6.1. Ex Post and Ex Ante Forecasts _..................................................... 106

6.2. Actual and Modeled Prices (Jan-95 to Dec-04) ................................ 107

7.1. Probabilities for Copper Prices to be

Greater than Specific Strike Prices _.......................................... 115

7.2. Bull Spread Using Call Options, Jan-06, X: 3100/3200 …................ 118

7.3. Bull Spread Using Call Options, Jan-07, X: 3100/3200 _.................... 118

7.4. Bull Spread Using Call Options, Jan-07, X: 3200/3300 …............... 119

7.5. Bull Spread Using Call Options, Jan-07, X: 3300/3400 …….............. 119

7.6. Bull Spread Using Put Options, Jan-06, X: 2200/2300 …................. 126

7.7. Bull Spread Using Put Options, Dec-06, X: 2600/2700 _................. 126

7.8. Bull Spread Using Put Options, Jan-07, X: 2300/2400 ......................... 126

7.9. Bull Spread Using Put Options, Dec-07, X: 2600/2700 _................ 126

7.10. Bear Spread Using Call Options, Dec-06, X: 3400/3300 _................. 133

7.11. Bear Spread Using Call Options, Dec-06, X: 3500/3400 _................ 133

7.12. Bear Spread Using Call Options, Dec-06, X: 3600/3500 ……......... 134

7.13. Bear Spread Using Call Options, Jan-06, X: 3600/3500 _................. 134

7.14. Butterfly Spread, Call Options, Jan-06, X: 3100/3200/3300 …........... 143

7.15. Butterfly Spread, Call Options, Jan-06, X: 3200/3300/3400 ….......... 143 
7.16. Butterfly Spread, Call Options, Jan-06, X: 3300/3400/3500 _.............. 143

7.17. Butterfly Spread, Call Options, Jan-06, X: 3400/3500/3600 _.............. 143

7.18. Butterfly Spread, Call Options, Jan-07, X: 3100/3200/3300 …........... 146

7.19. Butterfly Spread, Call Options, Jan-07, X: 3200/3300/3400 …............ 146

7.20. Butterfly Spread, Call Options, Jan-07, X: 3300/3400/3500 …............ 146

7.21. Butterfly Spread, Call Options, Jan-07, X: 3400/3500/3600 …............ 146

7.22. Butterfly Spread, Call Options, Dec-06, X: 3100/3200/3300 ….......... 153

7.23. Butterfly Spread, Call Options, Dec-06, X: 3200/3300/3400 …........... 153

7.24. Butterfly Spread, Call Options, Dec-06, X: 3300/3400/3500 …........... 153

7.25. Butterfly Spread, Call Options, Dec-06, X: 3400/3500/3600 …........... 153

7.26. Butterfly Spread, Call Options, Dec-07, X: 3100/3200/3300 …........... 156

7.27. Butterfly Spread, Call Options, Dec-07, X: 3200/3300/3400 ….......... 156

7.28. Butterfly Spread, Call Options, Dec-07, X: 3300/3400/3500 …......... 156

7.29. Butterfly Spread, Call Options, Dec-07, X: 3400/3500/3600 ….......... 156

7.30. Butterfly Spread, Put Options, Dec-06, X: 2300/2400/2500 ……....... 163

7.31. Butterfly Spread, Put Options, Dec-06, X: 2400/2500/2600 …............ 163

7.32. Butterfly Spread, Put Options, Dec-06, X: 2500/2600/2700 …............ 164

7.33. Butterfly Spread, Put Options, Dec-07, X: 2300/2400/2500 …........... 164

7.34. Butterfly Spread, Put Options, Dec-07, X: 2400/2500/2600 ……........ 164

7.35. Butterfly Spread, Put Options, Dec-07, X: 2500/2600/2700 …............ 164

7.36. Payoff Diagram of Strangle for Jan-07, X: 2700/3600 ….................... 169 


\section{ACKNOWLEDGEMENTS}

The help and guidance of many people contributed to the preparation of this thesis.

First of all, I would like to express my deep gratitude to my thesis advisor Professor Mark C. Roberts for his valuable advice, comments, criticisms, careful supervision, patience, and for all his support throughout this research. I am also very grateful to my academic advisor Professor Gary A. Campbell, who helped me to join the graduate program at Michigan Technological University, encouraged and enhanced my efforts, and helped me with valuable advice during the initial stages of this work.

I must also thank Professor Dean L. Johnson and Professor Brent J. Lekvin from whom I learned derivative securities, financial engineering and financial management through my attendance in their classes.

I am very thankful to all faculty and staff members of the School of Business and Economics. I would also like to thank to the members of my thesis committee, Professors Mark C. Roberts, Gary A. Campbell, Dean L. Johnson and Theodore J. Bornhorst for their valuable time, help and recommendations.

In addition, special thanks are due to following individuals for their valuable comments, assistance, and the provision of needed information and data:

- Mr. Maqsood Ahmed (Calyon Financial SNC of Credit Agricole Group)

- Ms. Ana Maria Rebelo (ICSG)

○ Mr. Rohan Ziegelaar (Sempra Metals Group) 
○ Mr. Robert J. Loewen

(International Metals Marketing, Commercial Advisory and Research Services)

- Mr. Peter Hollands (Bloomsbury Minerals Economics Ltd.)

- Mr. David Waite (CRM Associates)

- Mr. Batbayar Baldan

(Representative office in London, Erdenet Mining Company)

- Assistant professor Igor L. Kliakhandler

(Department of Mathematics, Michigan Technological University)

○ Mr. Daniel Edelstein (The U.S. Geological Survey).

Last but not least, thank you for my father Sandagdorj Enebish and mother Nadmid Banzar in Mongolia, for your love, enthusiasm, support and assistance. 


\begin{abstract}
Metals price risk management is a key issue related to financial risk in metal markets because of uncertainty of commodity price fluctuation, exchange rate, interest rate changes and huge price risk either to metals' producers or consumers. Thus, it has been taken into account by all participants in metal markets including metals' producers, consumers, merchants, banks, investment funds, speculators, traders and so on. Managing price risk provides stable income for both metals' producers and consumers, so it increases the chance that a firm will invest in attractive projects.

The purpose of this research is to evaluate risk management strategies in the copper market. The main tools and strategies of price risk management are hedging and other derivatives such as futures contracts, swaps and options contracts. Hedging is a transaction designed to reduce or eliminate price risk. Derivatives are financial instruments, whose returns are derived from other financial instruments and they are commonly used for managing financial risks. Although derivatives have been around in some form for centuries, their growth has accelerated rapidly during the last 20 years. Nowadays, they are widely used by financial institutions, corporations, professional investors, and individuals. This project is focused on the over-the-counter (OTC) market and its products such as exotic options, particularly Asian options.

The first part of the project is a description of basic derivatives and risk management strategies. In addition, this part discusses basic concepts of spot and futures (forward) markets, benefits and costs of risk management and risks and rewards of positions in the derivative markets.
\end{abstract}


The second part considers valuations of commodity derivatives. In this part, the options pricing model DerivaGem is applied to Asian call and put options on London Metal Exchange (LME) copper because it is important to understand how Asian options are valued and to compare theoretical values of the options with their market observed values.

Predicting future trends of copper prices is important and would be essential to manage market price risk successfully. Therefore, the third part is a discussion about econometric commodity models. Based on this literature review, the fourth part of the project reports the construction and testing of an econometric model designed to forecast the monthly average price of copper on the LME. More specifically, this part aims at showing how LME copper prices can be explained by means of a simultaneous equation structural model (two-stage least squares regression) connecting supply and demand variables. A simultaneous econometric model for the copper industry is built:

$$
\begin{aligned}
& \left\{\begin{array}{l}
\mathrm{Q}_{\mathrm{t}}^{\mathrm{D}}=\mathrm{e}^{(-5.0485)} \cdot \mathrm{P}_{(\mathrm{t}-1)}^{(-0.1868)} \cdot \mathrm{GDP}_{\mathrm{t}}^{(1.7151)} \cdot \mathrm{e}^{(0.0158)} \mathrm{IP}_{\mathrm{t}} \\
\mathrm{Q}_{\mathrm{t}}^{\mathrm{S}}=\mathrm{e}^{(-3.0785)} \cdot \mathrm{P}_{(\mathrm{t}-1)}^{(0.5960)} \cdot \mathrm{T}_{\mathrm{t}}^{(0.1408)} \cdot \mathrm{P}_{\mathrm{OIL}(\mathrm{t})}^{(-0.155)} \cdot \mathrm{USDI}_{\mathrm{t}}^{(1.2432)} \cdot \operatorname{LIBOR}_{(\mathrm{t}-6)}^{(-0.0561)} \\
\mathrm{Q}_{\mathrm{t}}^{\mathrm{D}}=\mathrm{Q}_{\mathrm{t}}^{\mathrm{S}}
\end{array}\right. \\
& P_{(t-1)}^{C U}=e^{(-2.5165)} \cdot G D P_{t}^{(2.1910)} \cdot e^{(0.0202) \cdot I P_{t}} \cdot T_{t}^{(-0.1799)} \cdot P_{\text {OIL }(t)}^{(0.1991)} \cdot U_{S D I}^{(-1.5881)} \cdot \operatorname{LIBOR}_{(t-6)}^{(0.0717)}
\end{aligned}
$$

Where, $Q_{t}^{D}$ and $Q_{t}^{S}$ are world demand for and supply of copper at time t respectively. $P_{(t-1)}$ is the lagged price of copper, which is the focus of the analysis in this part. 
$G D P_{t}$ is world gross domestic product at time $t$, which represents aggregate economic activity. In addition, industrial production should be considered here, so the global industrial production growth that is noted as $I P_{t}$ is included in the model. $T_{t}$ is the time variable, which is a useful proxy for technological change. A proxy variable for the cost of energy in producing copper is the price of oil at time $t$, which is noted as $P_{\text {OLL }(t)}$. USDI $_{t}$ is the U.S. dollar index variable at time $t$, which is an important variable for explaining the copper supply and copper prices. At last, $L_{I B O R_{(t-6)}}$ is the 6-month lagged 1-year London Inter bank offering rate of interest. Although, the model can be applicable for different base metals' industries, the omitted exogenous variables such as the price of substitute or a combined variable related to the price of substitutes have not been considered in this study.

Based on this econometric model and using a Monte-Carlo simulation analysis, the probabilities that the monthly average copper prices in 2006 and 2007 will be greater than specific strike price of an option are defined. The final part evaluates risk management strategies including options strategies, metal swaps and simple options in relation to the simulation results. The basic options strategies such as bull spreads, bear spreads and butterfly spreads, which are created by using both call and put options in 2006 and 2007 are evaluated. Consequently, each risk management strategy in 2006 and 2007 is analyzed based on the day of data and the price prediction model.

As a result, applications stemming from this project include valuing Asian options, developing a copper price prediction model, forecasting and planning, and decision making for price risk management in the copper market. 


\section{CHAPTER I:}

\section{INTRODUCTION}

Why should we hedge?

The principles of the market economy answer what and how to produce and for whom. How is this reflected in metal markets? Raw and semi fabricated materials, refined metals and metal end use products such as ingots, bars, wires, foils, sheets, strips, rods and tubes are produced using advanced technologies and traded for industrial and other needs. Metal markets have been highly competitive since ancient history because there are many producers and consumers. In metal markets, prices fluctuate in response to the global economic outlook, industrial production growth, international politics, market balances, exchange and interest rates, et cetera. Thus, no one could know exactly what will happen to metal markets and prices in the future even though analysts make outlook and price forecasts based on current economic, political and market conditions. If the market price goes down, it brings unexpected loss to metals' producers; in contrast, if the market price rises, consumers face increased costs. Of course, this "non-resistant" or unprotected condition against price changes will seriously impact future revenues of both metal buyers and sellers.

Furthermore, this uncertainty has a negative impact in state budget income and the economy of a country whose economy is strongly dependent on its metal industry. For instance, copper concentrate is the main export product from Mongolia and one third of the Gross Industrial Product (GIP) of Mongolia consists of copper concentrate export income. Hence, copper price is quite influential to the Mongolian economy. 
In 1994 and 1995, yearly average A-grade copper prices in the London Metal Exchange (LME) were $\$ 2,307$ and $\$ 2,935$ per tonne respectively. The budget deficit percentages in GDP of Mongolia were $5.4 \%$ and $0.9 \%$ in years above mentioned. However, these indices grew sharply in connection with a dramatic decline in copper prices in the following years and reached their highest level of $11.7 \%$ and $11.2 \%$ in 1998 and 1999 respectively when year average copper prices plunged to $\$ 1,654$ and $\$ 1,573$ per tonne. Approximately 38-40\% of Mongolian export income was from copper concentrate exports, but it fell to $27 \%$ in 1998 even though the amount of exported copper had not decreased. Generally, the reason for these bad consequences was the fall in copper prices in the global market. Chilean, South African, and Australian metal industries have a dominant role in their economies, so market price risk is a top concern when trading metals for these countries too. Moreover, not only these countries, but also every participant in the metal markets may need to carry out price risk management in order to reduce market price risk.

How is price risk management implemented and what is the final gain from it?

Market participants buy or sell their physical metals in the physical market through their regular channels. Alongside, they insure against their physical trading risks by using futures or options and other derivatives in the futures or over the counter (OTC) markets. These futures/forward and options contracts are issued by exchange brokers as Ring Dealing Members in the LME or Floor Brokers in NYMEX and COMEX.

Futures contracts are used for managing price risk and are liquidated by buying and selling them back before the (prompt) maturity date. Consequently, futures contract 
results can eliminate or reduce the price risk of physical trade, which is made by the cash settlement (spot) price.

Price risk management has the following benefits:

- It can maximize profits and minimize losses (reduce price risk) of hedgers because of protection against strong market price volatility;

- It gives an opportunity to plan future profits by stabilizing the net price of metals;

- It can help to predict and plan taxable income from metal industry to the state budgets.

However, risk management (hedging) is not always profitable because there can be a possible risk and costs occur if the price of the underlying commodity changes against a risk manager's expectation or if a risk manager chooses an inappropriate risk management tool (derivative). For example, a copper consumer who has a long forward position may lose if the price of copper significantly decreases in the future. A copper producer who has a short forward position may lose if the price of copper significantly increases in the future. Therefore, implementing price risk management can be an important part of the trading of metals and using a correct derivative at an appropriate time is a very important to risk managers.

One of the most actively traded base metals either in the exchanges or on the overthe-counter (OTC) markets is copper. Managing price risk in the copper market is very important to producers, consumers, speculators, traders, investment funds, banks etc. 
As seen from the figures below, trading volumes of futures and options (including traded average price options TAPOs) contracts are pretty volatile, but have been very high since 1997.

Figure (1.1): Monthly Trading Volumes of the LME Futures Contracts on Copper

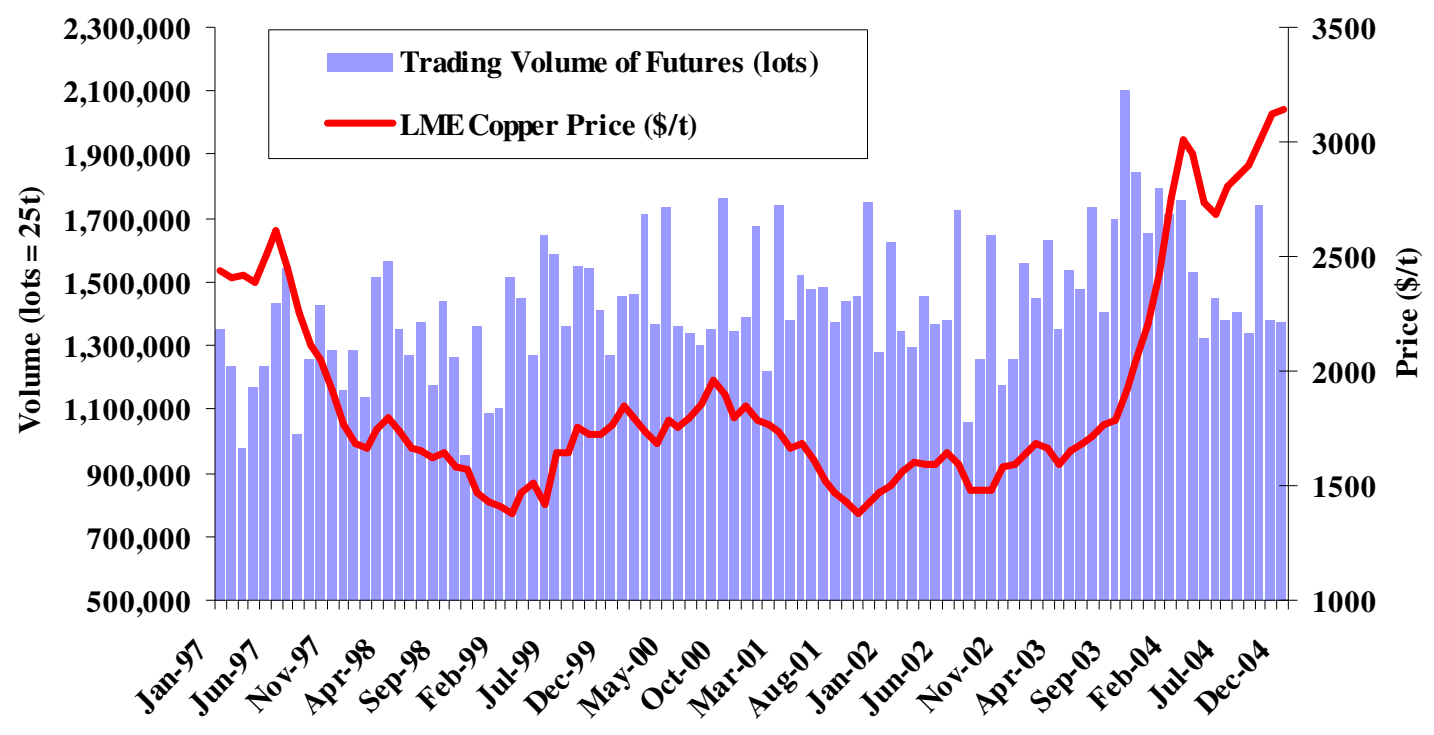

Figure (1.2): Monthly Trading Volumes of the LME Options Contracts on Copper

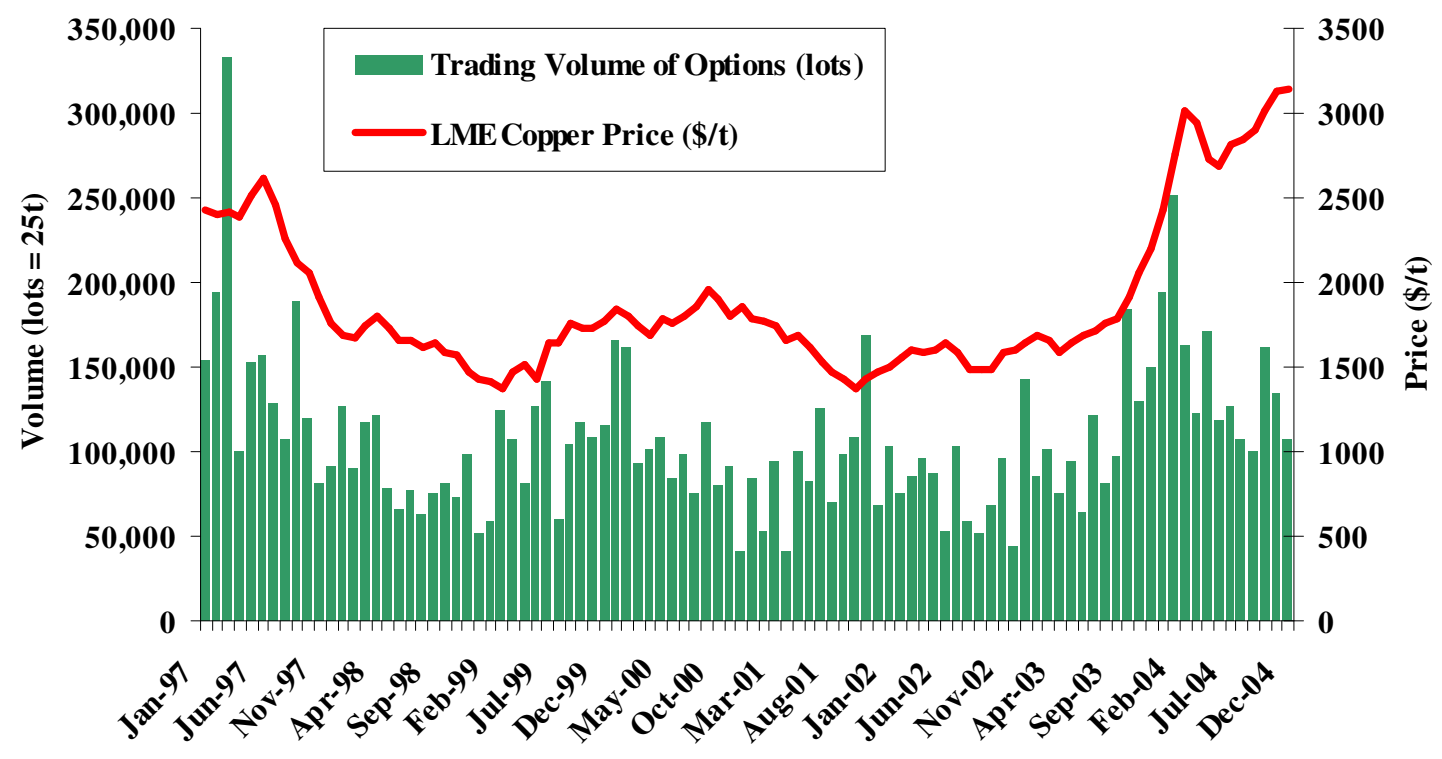


Everyday brokerage firms offer OTC products to their clients. The clients such as producers and consumers need to manage market price risk using these derivative products, but their risk management decisions will not be effective if they cannot do reasonable research regarding evaluation of risk management strategies. Due to this lack of experience and research, some clients have doubts about using derivative products for managing risk. Therefore, the main purpose of this research is to evaluate risk management strategies for copper in the OTC market including long hedge, short hedge, metal SWAPS, options contracts, options strategies such as bull spreads, bear spreads and butterfly spreads. The results are in 2006 and 2007 and based on the specific day of data because market variables change everyday; thus, brokerage firms regularly offer different indications.

In order to evaluate the OTC derivative strategies effectively, the LME copper price prediction model is developed by a simultaneous econometric model using two-stage least squares regression. The data observations consist of 120 months of historical data between January 1995 and December 2004. The model is developed based on the Cobweb model and a previously developed econometric model in the copper industry.

Using a Monte-Carlo simulation for the price prediction model, the probabilities for monthly average copper prices to be greater or less than specific strike prices are determined. These obtained probabilities are key factors for evaluating each risk management strategy.

Although, the results of the evaluations are only for the specific day of the data, copper producers, consumers, option traders and risk managers may use the model and methodologies in this thesis for any particular events and OTC indications in the future. 


\section{CHAPTER II:}

\section{DESCRIPTION OF RISK}

\section{MANAGEMENT STRATEGIES}

\subsection{BACKGROUND OF METAL EXCHANGES}

The traders of metals in the $19^{\text {th }}$ and the $20^{\text {th }}$ century were merchants, buying and selling physical metals. Actually, they earned their profits by transporting the metals from the places where metals were mined to the places where they were needed (The LME, 2001, p.2). In the $20^{\text {th }}$ century, activities of major metal exchanges such as the LME, the NYMEX and Shanghai Futures Exchange (SHFE) were developed as the world industrialized. The early development of the LME began because of Britain's role in developing the global industrial revolution. The LME began in 1877 and initially became the center of British metals trading. Today the LME is the largest derivative market for common non-ferrous metals and the most liquid metal exchange in the world. The main function of the LME is hedging, representing 75-85\% of turnover (Martinot, Lesourd, Morard, 2000, p.8). The LME services include reference pricing (daily official and settlement prices, real time and indicative market prices), hedging and delivery. The LME trades futures contracts for eight base metals: high grade primary aluminum, copper grade A, standard lead, special high grade zinc, primary nickel, tin, aluminum alloy, and North American Special Aluminum Alloy Contract (NASAAC). The approximate sailing time from Chile to London for transporting copper by ships was three months. Hence, this sailing time was the origin of the three months futures contract, which is the base of 
futures trading (Sigmon, 1997, p.11). In 1987 the old 3-month futures contract was extended. Contracts can now be traded up to 27 months for copper, primary aluminum, zinc and nickel and up to 15 months for lead, tin, and aluminum alloy. This is supported by the network of warehouses listed for the receipt of approved brands in locations in the UK, Continental Europe, Middle East, Singapore, Japan and the United States (The LME, 2001).

Traded Average Price Options (TAPO) was introduced to copper and primary aluminum in February of 1993, to nickel, zinc, aluminum alloy, lead and tin from October 2000, and to NASAAC from March 2002 on the LME.

The NYMEX is the largest physical commodity futures exchange in the world. The exchange include futures and options contracts for crude oil, gasoline, heating oil, natural gas, gold, silver, copper, aluminum, and platinum. COMEX is one division of the NYMEX. The COMEX division began trading gold futures in 1974 and the NYMEX Division platinum contract was launched in 1956. Aluminum futures opened its trade in May 1999 in the COMEX (NYMEX study material, 2003, p.1). Now, the COMEX division lists futures and options on gold, silver, copper and aluminum.

SHFE started its formal operation in December 1999. There are five contracts on the trading floor now and two of these contracts are metals: copper and aluminum. 


\subsection{SPOT VERSUS FUTURES MARKETS}

The physical market is made up of transactions, which result in the delivery of goods. The goods can be at any stage of production: ore concentrates or scrap metal through semi-fabricated and fabricated materials up to the end-use products, such as cable, electronic products, or even pots and pans.

Diagram (2.1): Copper Production Processes (Source: Claudio Agostini, 2002. p. 14)

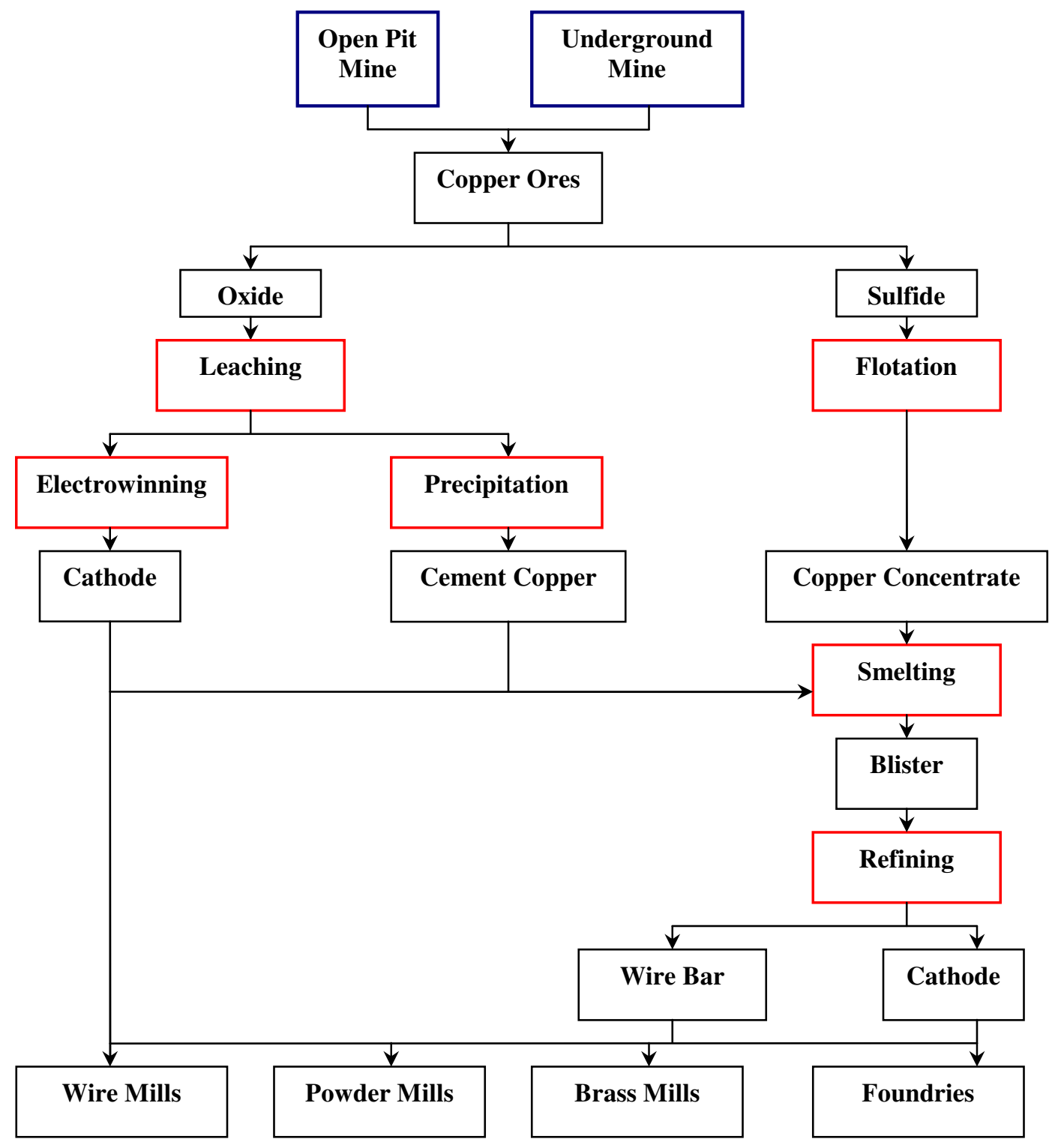


The metal passes through a number of processing stages before becoming a finished and end-use product. The processing stages of copper production are shown in the diagram above.

The production of refined copper consists basically of four steps including mining, concentrating, smelting and refining, according to a pyrometallurgical technology. However, the production steps are different in the low-cost hydrometallurgical technology (SX-EW) from traditional pyrometallurgical technology.

Diagram (2.2): Solvent Extraction-Electrowinning (SX-EW) Process

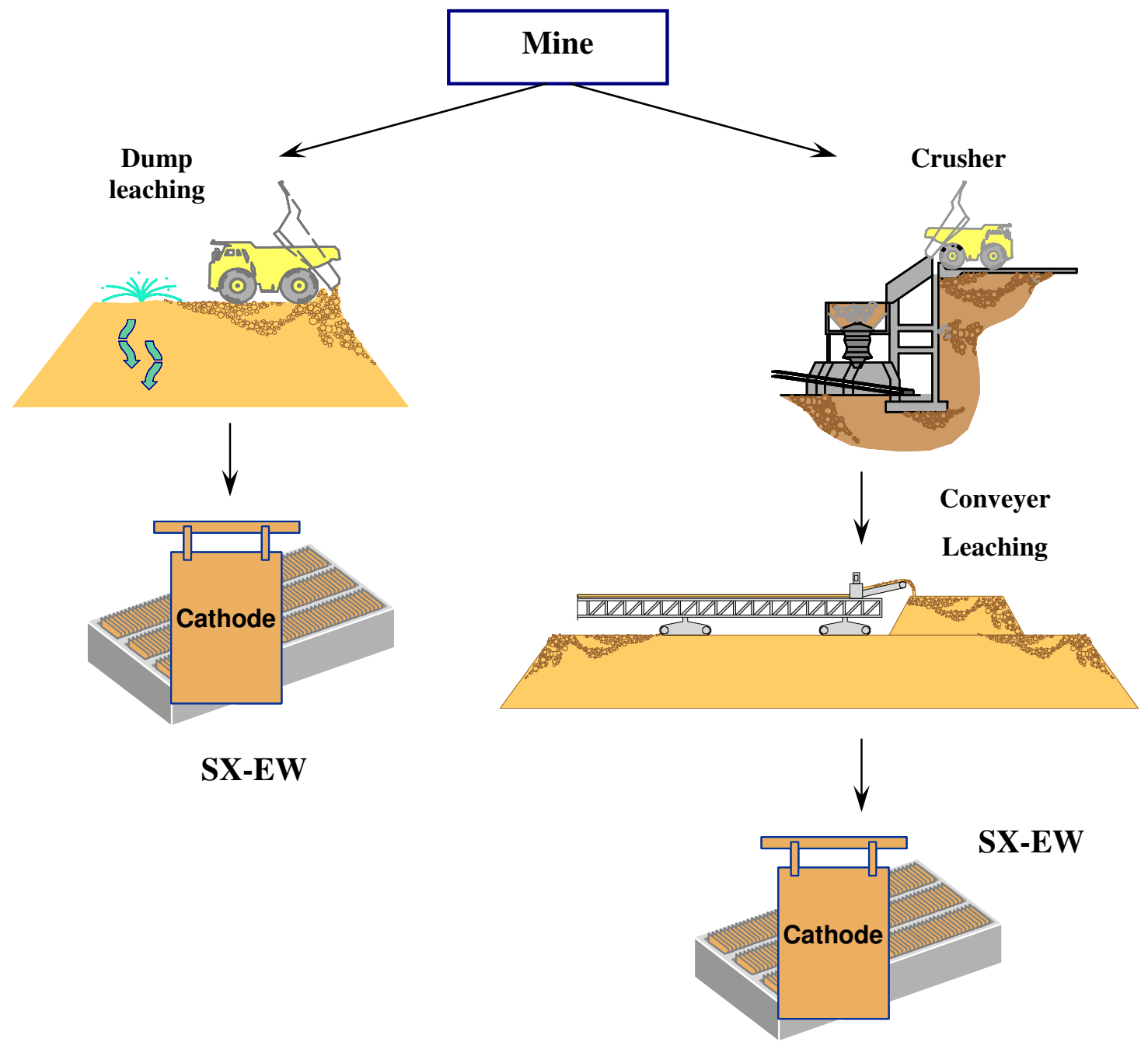

(Source: Phelps Dodge Corp., Presentation, 2002) 
As was mentioned before, many economic factors including production and consumption, international politics, governmental export controls, global wars, stockpiling policy, changing economic patterns, devaluations of currency, excess speculation relative to hedging, and structural changes within the metals industry have created considerable uncertainty to the future direction of the market and thus, to price irregularity. On the other hand, there can be a considerable time between each processing stage in copper production. Hence, there is a possible exposure to price risk for copper producers and consumers. In order to protect against such price risks, market participants can insure against their risks in physical metal exchanges through hedging. It may be prudent to hedge each physical transaction in the futures market.

Every ring dealer or broker member of the exchanges has clients from many sectors of the metal industry such as miners, smelters/refineries, rolling mills, extrusion plants, merchants, fabricators, distributors, consumers, secondary merchants, secondary smelters, banks, investment funds, speculators and so forth. These clients can hedge their physical transactions by entering into futures (forward) contracts on each exchange.

Only a small percentage (approximately $2 \%$ ) of LME contracts result in an actual delivery because traders generally offset their futures positions before the maturity dates. A buyer will liquidate by selling the contract and a seller will liquidate by buying back the contract.

Copper producers and consumers consider that a stable price leads to higher returns than a volatile price that fluctuates in response to short-term variations in demand and supply. The price of copper substantially affects the profitability of a copper producer. The producer will make good profits when demand for copper and prices are high. 
Nevertheless, if copper prices fall, there is the risk that the market price for copper will be lower than the cost of production. If the market price cannot cover the cost of production for a long time, a bankruptcy can result for the producer. Therefore, hedging is one way to protect against a price risk. In the case of copper producers, they hedge their position against the risk of a fall in the price of copper.

\subsection{DESCRIPTION OF BASIC DERIVATIVES}

\section{A) Futures (forward) contracts and Hedging:}

The futures market is the trade of contracts for the future delivery of goods. In theory, futures contracts are determined as an agreement to buy or sell a standard quantity of a specified asset on a fixed date at a price agreed today. The futures contracts are standardized and traded on exchanges. Therefore, futures contracts are guaranteed by exchange clearinghouse. In addition, there are daily settlements; thus, futures contract traders need to have a margin account to prevent credit risks.

By contrast, forward contracts are not standardized, so they are traded between any two parties like financial institutions and their corporate clients. Non-standardized derivatives such as forward contracts are traded in the over-the-counter (OTC) market, which is an important alternative to exchanges. The OTC market operates through a telephone and computer based network of dealers. A single payment is paid at the maturity in forward contracts, so there is usually some credit risks.

Futures contracts have been used to manage cash market price risk for more than a century. In a futures market, there are two parties to every contract. One party agrees to 
buy and take delivery in the future and the other party agrees to sell and make delivery in the future. The party agreeing to buy is said to have a long position and the party agreeing to sell has a short position.

Hedging is a form of price insurance, which allows a market participant (producers and consumers) to lock in prices and reduce the potential for unanticipated loss. This means, a hedger makes his/her position certain and transfers a spot market price risk to another party by forward dealing. Therefore, the economic purpose of the futures market is to provide an arena for transferring risk among market participants. The hedger takes an opposite position in the futures market from that in the spot market. The profits in one market are offset by losses in another (Labys, Granger, 1970, p.21). A short hedge is the case where a hedger is long in the spot market and short in the futures market in order to protect an existing holding. In contrast, a long hedge refers to when a hedger is short in the spot market and long in the futures market to protect an anticipated holding.

There are two types of hedges: a price fix hedge or an offset hedge. A price fix hedge is a trade of futures contracts to fix the future purchase or sale price of a metal. An investor; therefore, wishes to profit from a directional view in the future price movements by fixing today the price for the future. According to the price fix hedge, there are two cases for copper producers whether to buy forward contracts or not.

- Case 1) If metal producers feel that prices will fall between now and the time physical contract matures, then they sell at a forward price today and buy back at a cash settlement price at the maturity, so the selling price is fixed.

- Case 2) If metal producers feel that prices will rise between now and the time physical contract matures, then there is no need to do a hedge. 
For copper consumers, the cases would be:

- Case 1) If metal consumers feel that prices will rise between now and the time physical contract matures, then they buy at a forward price today and sell back at a cash settlement price at the maturity, so the purchasing price is fixed.

- Case 2) If metal consumers feel that prices will fall between now and the time physical contract matures, then there is no need to do a hedge.

An offset hedge establishes an equal and opposite position in the futures market to an existing position in the spot market. Hence, a short physical position is offset by a long futures position, and a long physical position is offset by a short futures position. The offset hedge positions are typically liquidated before their maturity dates.

Typically, everyday ring dealing members and floor brokers value futures prices based on three months futures price (3-M) and carrying charges including interest rates, insurance, and storage costs in official warehouses. Actually, the forward price should equal the three-month price plus these carrying charges. Table (2.1) represents the forward prices indications, which were valued on $17^{\text {th }}$ of March, 2003 by Calyon Financial SNC, one of the ring dealing members (category I) of the LME. The following example shows a copper producer's price fix hedging operation.

Example 1: Suppose that the Alumbrera copper mine decided to hedge 520 lots (1 lot equals 25 tonnes) of A-grade copper in concentrate on $17^{\text {th }}$ of March, 2003. Outokumpu agreed to buy this amount of copper in concentrate for its copper wire production at a spot price. Assume May-2003 was the delivery month, so it was also used as the 
quotation month (in this case, the quotation period is the same as the delivery month) which is the pricing period. Assume the Alumbrera settled in full on 2 June 2003, two days after the quotation period ended on 31 May 2003 as shown by table (2.1).

Table 2.1:

The LME Forward Prices (Calyon)

\begin{tabular}{cc}
\hline $17 / 03 / 2003$ & Cash $=\$ 1655.00 / t$ \\
\hline Month & Forward price U.S. \$/t \\
\hline Mar. 2003 & $1,656.00$ \\
\hline Apr. 2003 & $1,662.00$ \\
\cline { 1 - 1 } May. 2003 & $\mathbf{1 , 6 6 9 . 0 0}$ \\
\cline { 1 - 1 } Jun. 2003 & $1,673.75$ \\
\cline { 1 - 1 } Jul. 2003 & $1,676.50$ \\
\hline Aug. 2003 & $1,680.25$ \\
\hline Sep. 2003 & $1,684.00$ \\
\hline Oct. 2003 & $1,686.00$ \\
\hline Nov. 2003 & $1,688.00$ \\
\hline Dec. 2003 & $1,690.00$ \\
\hline
\end{tabular}

Table 2.2:

The Example Data

\begin{tabular}{ll}
\hline Metal type & Copper \\
Tonnage & 520 lots \\
Quotation period & May-03 \\
Payment made & 2-Jun-03 \\
Price available for 2-Jun-2003 & $\$ 1,669.00 / \mathrm{t}$ \\
\hline
\end{tabular}

Note: 520 lots equal to 13,000 tonnes of copper in concentrate. If Alumbrera's copper content in concentrate was $28 \%$, the total hedged amount of copper concentrate would be 46,429 tonnes through this contract.

On 17 March, the Alumbrera made the first LME contract with Calyon in the OTC market. The short forward position (sell forward) with prompt date of 2 June 2003 was opened on that day at the price of $\$ 1,669.00 / t$ for 520 lots of copper (see table (2.1)). Suppose the Alumbrera's production cost was $\$ 1,025.00 / t$ in 2003 . On the settlement date of 2 June, the Alumbrera made the second LME contract with Calyon. In other words, the mine closed out the short forward position in the OTC market by buying back 520 lots of copper at an average cash settlement price (ACSP) of the quotation period May 2003. The average cash settlement price (ACSP) of May-03 was \$1,648.28/t on the LME. The price difference between these two LME contracts is the nominal profit or loss.

In the physical (spot) market, the Alumbrera sold Outokumpu 520 lots of copper at the ACSP of May 2003, which was the quotation period as mentioned in table (2.2). The difference between the cost of production and the ACSP of the quotation period is the 
profit or loss in the physical (spot) market. Therefore, the Alumbrera's physical and forward contracts would be as follows:

Table 2.3: The Alumbrera's Price Fix Hedging Operation with the LME

\begin{tabular}{|c|c|c|c|}
\hline \multicolumn{2}{|c|}{ The LME contract with Calyon Financial } & \multicolumn{2}{|c|}{ PHYSICAL contract with Outokumpu } \\
\hline \multicolumn{2}{|c|}{$\begin{array}{l}\text { On } 17 \text { March 2003: } \\
\text { - (The LME Contract 1) } \\
\text { Short forward 520 lots (CU) @ \$1,669.00/t } \\
\text { Prompt 2-June-2003. }\end{array}$} & \multicolumn{2}{|c|}{$\begin{array}{l}\text { On } 17 \text { March 2003: } \\
\text { - Production cost of copper in } \\
\text { concentrate was } \$ 1,025.00 / \mathrm{t}^{* *} \text {. }\end{array}$} \\
\hline \multicolumn{2}{|c|}{$\begin{array}{l}\text { - } \text { (The LME Contract } 2) \\
\text { Buy back } 520 \text { lots @ ACSP* of May } 2003 . \\
\text { (ACSP of May-03= } \$ 1,648.28 / \text { t) }\end{array}$} & \multicolumn{2}{|c|}{$\begin{array}{l}\text { - }(\text { Physical Contract) } \\
\text { Short Sell } 520 \text { lots @ ACSP of May } 2003 . \\
(\text { ACSP of May- } 03=\$ 1,648.28 / t)\end{array}$} \\
\hline Nominal LME profit & $\$ 20.72 / \mathrm{t}$ & Physical profit & $\$ 623.28 / t$ \\
\hline \multicolumn{2}{|c|}{ Total profit (net effects of the hedge) } & \multicolumn{2}{|c|}{$\$ 644.00 / t$} \\
\hline \multicolumn{2}{|c|}{$*$ ACSP $=$ Average Cash Settlement Price } & \multicolumn{2}{|c|}{ ** Assumed cost of production } \\
\hline
\end{tabular}

Settlement on 2 June 2003: the difference between the LME contract [1] and the LME contract [2].

* If the average price in the quotation period was less than $\$ 1,669.00 / t$ (forward price), Calyon Financial would pay Alumbrera the difference;

If the average price in the quotation period was more than $\$ 1669.00 /$ t (forward price), Alumbrera would pay Calyon Financial the difference.

Net result: Irrespective of whether the copper price goes up or down in the quotation period, Alumbrera receives $\$ 1,669.00 /$ t (fixed price) when hedge is taken out. 
In this example, the brokerage firm (Calyon Financial) would pay Alumbrera $\$ 20.72 / t$, the difference between the forward price and the ACSP of May-03. Because of this hedging operation, the Alumbrera mine profited by $\$ 20.72$ per tonne during this period and this totaled $\$ 269,360$ excluding commission charges. The total gain would be $\$ 8,372,000$ with this hedging and $\$ 8,102,640$ if hedging had not been used. In this example, the costs of hedging including margin requirements, commission and transaction costs are ignored.

In fact, commodity futures prices are the interplay between a number of factors including supply and demand in the spot markets, interest rates, transportation and storage costs etc. The principle of price risk management is based on the term "basis", which is the numerical difference between a futures price and a spot price (cash market price). The basis is not constant because the relationship between supply and demand affects the movements in the basis. If the futures prices are higher than spot prices (positive basis), the market is said to be in contango (at a premium), otherwise (negative basis), in backwardation (at a discount). When there is a lack of supply or very little amount of inventory, a contango market can be altered into a backwardation. This relationship is shown by figure (2.1).

The market moves into contango as inventories (enough material to be sold) rise. The copper market has been in backwardation since the second half of 2003 due to its lack of supply and low level of inventories on the exchanges. 
Figure (2.1): The Relationship between Contango/Backwardation markets and Inventories

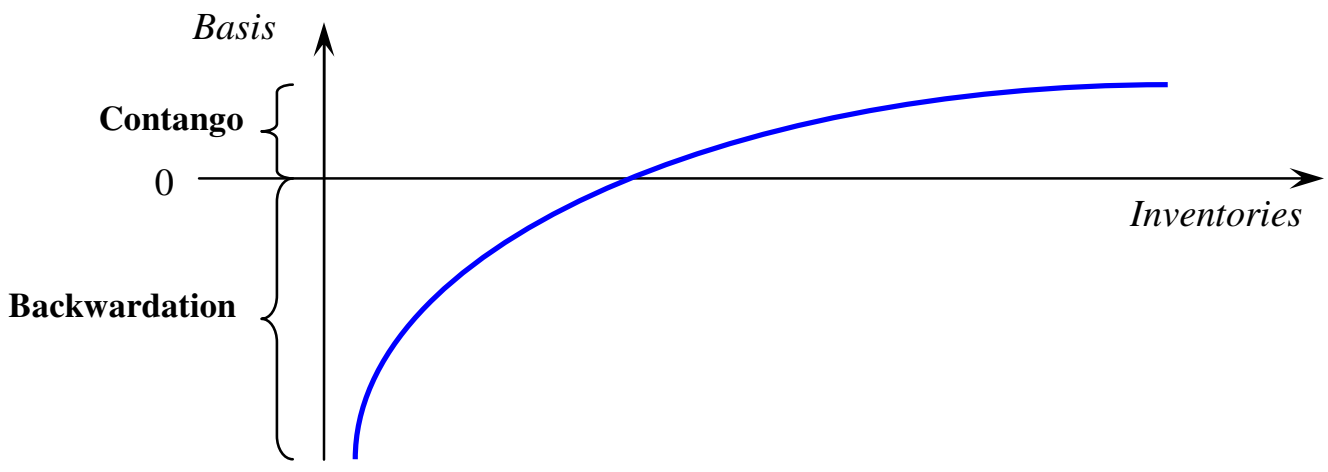

Example 2: To illustrate an offset hedge, consider a hedger who expects the price of copper to decrease and protects against this risk of a price fall. In this case, the hedger takes a short position (selling futures contract) in the futures market and a long (buying copper) in the spot market initially (on 24 January). Before the maturity of the futures contract, the hedger offset his/her position on 20 March by shorting back (selling copper) in the spot and by taking a long position (buying futures contract) in the futures markets. As can be seen from the table below, $\$ 70 / t$ fall or loss in the price of cash copper has been entirely offset by a $\$ 90 / \mathrm{t}$ gain in the futures market, so the net gain to the hedger is $\$ 20 / t$ resulting from the hedging operation.

Table 2.4: Basis Risk versus Outright Risk

\begin{tabular}{ccc}
\hline Date & Spot Market & Futures Market \\
\hline 24 January & Long @ \$2,350/t & Short @ \$2,420/t \\
20 March & Short @ \$2,280/t & Long @ \$2,330/t \\
\hline & Loss: $(\$ 70 / \mathrm{t})$ & Profit: $\$ 90 / \mathrm{t}$ \\
\hline Net gain/loss & & $\$ 20 / \mathrm{t}$ \\
\hline
\end{tabular}




\section{B) Metal Swaps (Fixed purchase and sale):}

Metal swaps are non-standardized derivatives in the OTC market. Like a price fix hedge, they are settled against the average of the LME cash settlement prices. However, metal swaps consist of monthly forward purchases or sales that are settled against the average cash settlement prices each month until the final maturity of the swaps. Thus, a producer makes fixed price sales each month, which are settled against the monthly average of the LME cash settlement prices (floating prices). A consumer makes fixed price purchases each month.

Hedgers can use metal swaps in order to make their future profits constantly guaranteed for a specific time at an adequate price for them if commodity prices are very volatile and the commodity market trend is likely to be uncertain. The following example shows the basic procedures of metal swaps for a copper consumer.

Example 3: Suppose that copper alloy products and copper tube producer Swissmetal needed to purchase 80 lots $(2,000$ tonnes $)$ of refined copper for its tube production each month starting from July 2004 until December 2004 for six months. The monthly average cash price of copper on the LME was settled at \$3,008.72/t in April 2004, which was the 103-month highest since September 1995 (\$3,036.84/t). Because of this bullish trend in the global copper market in the second half of 2004, Swissmetal decided to hedge against the risk of price increases using monthly swap contracts. Assume that the fixed purchase prices (swap) were offered in May 2004 by a LME floor broker as $\$ 2,900 / t$ in July 2004 through December 2004 and the Swissmetal purchased monthly swaps for this time period at this offered price $\$ 2,900 / t$ in May 2004. Therefore, the tube producer agreed to 
buy 80 lots of refined copper each month at $\$ 2,900 / t$ in July-04 through December-04. In other words, the tube producer locked its purchasing prices at $\$ 2,900$ in the second half of 2004. These swaps were settled each month against the monthly average cash settlement prices (MACSP) of the LME. Hence, at the end of each month, Swissmetal sold back 80 lots of refined copper at the MACSP between July and December of 2004. The table below shows the outcomes of the metal swaps contracts for Swissmetal.

Table 2.5: The Results of Metal Swaps Contract for 6 Months

\begin{tabular}{|l|c|c|c|c|c|c|}
\hline & \multicolumn{6}{|c|}{$\mathbf{2 0 0 4}$} \\
\cline { 2 - 7 } & Jul. & Aug. & Sep. & Oct. & Nov. & Dec. \\
\hline Long Swap (\$/t) & 2,900 & 2,900 & 2,900 & 2,900 & 2,900 & 2,900 \\
Short Sell @ MACSP & $2,686.70$ & $2,810.74$ & $2,846.10$ & $2,894.86$ & $3,012.24$ & $3,122.80$ \\
\hline Swap profit/loss & $\mathbf{- 2 1 3 . 3 0}$ & $\mathbf{- 8 9 . 2 6}$ & $\mathbf{- 5 3 . 9 0}$ & $\mathbf{- 5 . 1 4}$ & $\mathbf{1 1 2 . 2 4}$ & $\mathbf{2 2 2 . 8 0}$ \\
\hline
\end{tabular}

In the physical market, Swissmetal purchased 80 lots of copper each month at the MACSP. As shown in the table above, the fixed purchases at $\$ 2,900 / t$ were not profitable for Swissmetal between July and October because the monthly average cash settlement prices were settled below the swap prices of $\$ 2,900 /$ t. Moreover, Swissmetal could not eliminate price risks during the first 4 months, but it reduced price risks in the last 2 months because the tube producer profited in November and December 2004.

In this example, Swissmetal would have negative cash flows in the first 4 months, but positive cash flows in the last 2 months. If the effective interest rate is assumed to be $1.25 \%$ per month during this period, the net present value of these cash flows would be negative $(\$ 41.76 / \mathrm{t})$ in May 2004, which is the contract date. As a result, the entire swaps would not be profitable for Swissmetal in this example. The costs of hedging issues will be discussed later. 


\section{C) Options contracts:}

Options contracts are rights, but not the obligation, to buy or sell an asset at a specified price within a certain date. Basically there are three styles of options, including European, American and exotic options. An American option can be exercised on any day up through the expiration date, but European style and some kinds of exotic options are exercised only at the maturity date.

There are two types of options: a call option and a put option. An option that gives the option holder the right to buy an asset is referred to as a call, an option that gives a holder the right to sell an asset is called a put option. The option buyer pays the seller (writer) a sum of money called the option price or premium. The fixed price at which the option holder can either buy or sell the asset is called the exercise or strike price. A call option in which the asset price at the maturity $\left(S_{T}\right)$ exceeds the exercise price $(X)$ (i.e. $\left.S_{T}>X\right)$ is said to be in-the-money and if the asset price at the maturity is less than the exercise price (i.e. $\mathrm{S}_{\mathrm{T}}<\mathrm{X}$ ), the call option is said to be out-of-the-money. By contrast, a put option is said to be in-the-money, if the asset price at the option's maturity is less than the exercise price (i.e. $\mathrm{S}_{\mathrm{T}}<\mathrm{X}$ ). If the asset price at the option's maturity is more than the exercise price (i.e. $\mathrm{S}_{\mathrm{T}}>\mathrm{X}$ ), the put option is said to be out-of-the-money. If the asset price at the maturity is equal the exercise price (i.e. $S_{\mathrm{T}}=\mathrm{X}$ ), the option is said to be at-the-money. In-the-money options are exercised, but out-of-the-money options are never exercised.

Exotic options are traded in the over-the-counter (OTC) market, which is now a very large and widely used alternative market to the exchange. These options offer different types of payoffs. One of the widely used exotic options between financial institutions and participants in the metals industry is an average price option known as an Asian option. 
An Asian option is an option where the payoff is based on the average price attained by the underlying asset during the life of the option or over a specified period of time, which does not have to correspond to the life of the option. Asian options can take the form of either a call or a put (Chance, 2001, p. 663).

\subsection{THE COSTS OF HEDGING}

Hedging is not always profitable because changes in the basis result in either a potential profit (opportunity) or loss (threat) to investors and risk managers (hedgers). If the prices of the underlying commodity move in the other direction from a hedger's expectation, there can be possible risks and costs (loss) of hedging. Of course there is not a perfect relationship between cash and futures markets, so there is no such thing as a perfect hedge. The possible risks and costs of hedging occur when the market prices change against the trend expected by a hedger. The following examples illustrate this case.

Example 4: Again using table (2.1) on page 14. The table indicates forward price valuations on 17 March 2003. Based on this information, copper producers and consumers could hedge in the OTC market. The first column of table (2.6) shows different maturity dates of the forward contracts. The forward price valuations and the monthly average cash settlement prices (MACSP) on the LME are shown in the second and the third columns of the table respectively. The fourth column indicates all possible profits and losses of price fix hedging operation for a copper producer on 17 March 2003. 
A producer initially takes a short forward position in the OTC market in order to hedge against a risk of a fall in prices. When the forward contract matures, the producer closes his/her short forward position by buying back at the LME average cash settlement price of the delivery month. In contrast, a copper consumer initially takes a long position in the OTC market and closes his/her long forward position by short selling at the LME average cash settlement price of the delivery month. The potential profits and losses of price fix hedge for a copper consumer on the data date are shown in column six.

Table 2.6: A Summary Result of Price Fix Hedge on 17 March 2003

\begin{tabular}{|c|c|c|c|c|c|c|}
\hline \multicolumn{3}{|c|}{ Valuations: 17 March 2003} & \multicolumn{4}{|c|}{ Cash $=\$ 1,655.00 / t$} \\
\hline \multirow[t]{2}{*}{ Month } & \multirow{2}{*}{$\begin{array}{c}\text { Forward price } \\
(\text { U.S. } \$ / t)\end{array}$} & \multirow{2}{*}{$\begin{array}{l}\text { MACSP* } \\
\text { (U.S. \$/t) }\end{array}$} & \multicolumn{4}{|c|}{ Hedge Profit/Loss } \\
\hline & & & Short Forward & Gain/Loss & Long Forward & Gain/Loss \\
\hline Mar. 2003 & $1,656.00$ & $1,658.98$ & -2.98 & Loss & 2.98 & Gain \\
\hline Apr. 2003 & $1,662.00$ & $1,587.48$ & 74.53 & Gain & -74.53 & Loss \\
\hline May. 2003 & $1,669.00$ & $1,648.28$ & 20.72 & Gain & -20.72 & Loss \\
\hline Jun. 2003 & $1,673.75$ & $1,686.50$ & -12.75 & Loss & 12.75 & Gain \\
\hline Jul. 2003 & $1,676.50$ & $1,710.00$ & -33.50 & Loss & 33.50 & Gain \\
\hline Aug. 2003 & $1,680.25$ & $1,760.28$ & -80.03 & Loss & 80.03 & Gain \\
\hline Sep. 2003 & $1,684.00$ & $1,789.52$ & -105.52 & Loss & 105.52 & Gain \\
\hline Oct. 2003 & $1,686.00$ & $1,920.54$ & -234.54 & Loss & 234.54 & Gain \\
\hline Nov. 2003 & $1,688.00$ & $2,055.43$ & -367.43 & Loss & 367.43 & Gain \\
\hline Dec. 2003 & $1,690.00$ & $2,201.29$ & -511.29 & Loss & 511.29 & Gain \\
\hline
\end{tabular}

As can be seen from table (2.6), a producer's price fix hedge would only be profitable for April-03 and May-03 contracts. Except for these two contracts, all possible hedges would not be profitable because the producer would pay the difference between the forward prices and the MACSP (negative values in column 4) to the counterparty (brokerage firm) if the producer hedged on 17 March 2003. However, the situation would be in reverse for a copper consumer. If a copper buyer used price fix hedge on 17 March 2003, he/she would gain from all possible forward contracts through 2003, except April03 and May-03 contracts. 
Therefore, example 4 shows that hedging (in this example, a price fix hedge) can result in either profit or loss. Example 3 showed the costs of hedging in case of metal swaps contracts.

Options can also result some loss. For example, if an option is out-of-the-money, the option will not be exercised and the option holder's loss will be the option price (premium) because the holder pays the premium to the option writer.

Example 5: Suppose that the Los Pelambres copper mine decided to buy an Asian put option in the OTC market on 06 January 2005. The purpose of buying a put option was to avoid exposure to a price fall. The LME floor broker Sempra Metals Group valued the put options for February through December 2005 as shown by table (2.7). Table (2.8) shows the monthly average cash settlement prices of the LME between February and August of 2005.

Table 2.7:

Producer Put Option Indications for Feb-Dec 05

\begin{tabular}{cc}
\hline Valued on 06 January 2005 & Cash $=\$ 3,095.00 / t$ \\
\hline Strike U.S. \$/t (X) & Premium U.S. \$/t* \\
\hline $2,350.00$ & 42.00 \\
\hline $2,450.00$ & 61.00 \\
\hline $2,550.00$ & 86.00 \\
\hline $2,650.00$ & 117.00 \\
\hline $2,750.00$ & 156.00 \\
\hline Source: Rohan Ziegelaar, "Copper producer OTC indications", \\
Sempra Metals Group, January 6, 2005 \\
\hline *The premium increases with volatility, time to expiry and proximity of \\
strike to market price.
\end{tabular}

Table 2.8: Monthly Average Prices (LME) Month MACSP U.S $\$ / \mathbf{t}\left(\mathbf{S}_{\mathrm{AVG}}\right)$

\begin{tabular}{cc} 
& $\mathbf{U} \mathbf{S} \$ \mathbf{t}\left(\mathbf{S}_{\mathbf{A V G}}\right)$ \\
\hline Feb-05 & $3,253.70$ \\
Mar-05 & $3,379.60$ \\
Apr-05 & $3,394.48$ \\
May-05 & $3,249.10$ \\
Jun-05 & $3,524.07$ \\
Jul-05 & $3,614.21$ \\
Aug-05 & $3,797.75$ \\
\hline
\end{tabular}

The indications above are monthly settled Asian options. In other words, the options are settled against the monthly average cash settlement prices. The Los Pelambres needs to pay the premium to the Sempra Metals for insuring against a fall in prices. 
Therefore, the option holder's (Los Pelambres) net cash flow at time zero (on 06 January 2005) is always negative. Assume that the mine (Los Pelambres) bought put option at a strike $\$ 2,550$ with April-05 maturity. In this case, the mine would immediately pay $\$ 86$ as the premium to the brokerage firm (Sempra Metals) at time zero (on 06 January 2005). The expected payoff from this Asian put option is maximum of $\left\{0, \mathrm{X}-\mathrm{S}_{\mathrm{AVG}}\right\}$, where $\mathrm{X}$ is the exercise price and $\mathrm{S}_{\mathrm{AVG}}$ is the average value of the underlying asset calculated over a predetermined averaging period. This predetermined averaging period is April 2005 in this example (i.e. $S_{\mathrm{AVG}}=$ MACSP of April-05). The mine's expected payoff at the option's maturity; therefore, is:

1) If $S_{A V G}<X$, then the payoff $=\left\{X-S_{A V G}\right\}>0$ (the option is in-the-money)

2) If $S_{A V G}>X$, then the payoff $=0$ (the option is out-of-the-money)

The average cash settlement price of April-05 was $\$ 3,394.48$, which greater than the strike price $(\$ 2,550)$; hence, the option was not exercised and the expected payoff would be zero. Therefore, the Los Pelambres mine would lose because it had negative cash flow at time zero and zero cash flow in the future. As can be seen from table (2.8), the MACSP of the LME in the predetermined averaging months were all settled at values that are greater than the available strike prices on the valuation date of 06 January 2005 , so it indicates that all of these Asian put options were not exercised. Hence, producer put option holders at these strike prices in this example would lose the premium.

In conclusion, there can be potential costs (loss) if the prices of the underlying commodity move and are settled against our risk management strategy and expectations of price fluctuations. Therefore, choosing and using a correct derivative at an appropriate time is very important. 


\subsection{RISKS AND REWARDS OF DERIVATIVE MARKET POSITIONS}

In this part, risks and rewards of different positions in the derivative market will be summarized. Let us start with futures (forward) contracts.

\begin{tabular}{|c|c|c|}
\hline Notations: & $\mathrm{S}_{0}=$ the spot price at time zero & $\mathrm{S}_{\mathrm{t}}=$ the spot price at time $\mathrm{t}$ \\
\hline
\end{tabular}

Table 2.9: Payoff Matrix of an Offset Hedging

\begin{tabular}{|c|c|c|c|c|}
\hline & \multicolumn{2}{|c|}{ A Long Hedge } & \multicolumn{2}{|c|}{ A Short Hedge } \\
\hline & $\begin{array}{c}\text { Spot } \\
\text { market }\end{array}$ & $\begin{array}{c}\text { Futures } \\
\text { market }\end{array}$ & $\begin{array}{c}\text { Spot } \\
\text { market }\end{array}$ & $\begin{array}{c}\text { Futures } \\
\text { market }\end{array}$ \\
\hline Time 0 & Short $\left(+S_{0}\right)$ & Long $\left(-\mathrm{F}_{0}\right)$ & Long $\left(-\mathrm{S}_{0}\right)$ & Short $\left(+\mathrm{F}_{0}\right)$ \\
\hline Time $\mathrm{t}$ & $\operatorname{Long}\left(-S_{t}\right)$ & Short $\left(+F_{t}\right)$ & Short $\left(+S_{t}\right)$ & $\operatorname{Long}\left(-F_{t}\right)$ \\
\hline Net Effect of Hedge & \multicolumn{2}{|c|}{$\begin{array}{l}S_{0}-S_{t}-F_{0}+F_{t}= \\
=\left(S_{0}-F_{0}\right)-\left(S_{t}-F_{t}\right) \\
=\left(\mathbf{F}_{t}-S_{t}\right)-\left(\mathbf{F}_{\mathbf{0}}-\mathbf{S}_{\mathbf{0}}\right)>\mathbf{0}\end{array}$} & \multicolumn{2}{|c|}{$\begin{array}{l}S_{t}-S_{0}+F_{0}-F_{t}= \\
=\left(S_{t}-F_{t}\right)-\left(S_{0}-F_{0}\right) \\
=\left(\mathbf{F}_{\mathbf{0}}-S_{0}\right)-\left(\mathbf{F}_{t}-S_{t}\right)>\mathbf{0}\end{array}$} \\
\hline
\end{tabular}

A long hedger makes a profit if backwardation $\left(S_{t}>F_{t}\right)$ decreases or if contango $\left(F_{t}>S_{t}\right)$ increases because the net effect of the hedge (gain/loss) is equal to $\left\{\left(\mathbf{F}_{\mathbf{t}}-\mathbf{S}_{\mathbf{t}}\right)-\right.$ $\left.-\left(\mathbf{F}_{\mathbf{0}}-\mathbf{S}_{\mathbf{0}}\right)\right\}$, which should be greater than zero. By contrast, a short hedger makes a profit if backwardation $\left(S_{t}>F_{t}\right)$ increases, or contango $\left(F_{t}>S_{t}\right)$ declines. In this case, the net effect of the hedge will be $\left\{\left(\mathbf{F}_{\mathbf{0}}-\mathbf{S}_{\mathbf{0}}\right)-\left(\mathbf{F}_{\mathbf{t}}-\mathbf{S}_{\mathbf{t}}\right)\right\}$. The outcomes of different futures positions for offset hedging with different market situations are shown in the diagram below. The long and short positions are the positions in the futures market.

As can be seen from the diagram below, a long hedger will gain as $\left(F_{t}-S_{t}\right)$ approaches $+\infty$ in a contango market, but $\left(\mathrm{S}_{\mathrm{t}}-\mathrm{F}_{\mathrm{t}}\right)$ approaches zero in a backwardation market. In contrast, a short hedger gains as $\left(\mathrm{F}_{\mathrm{t}}-\mathrm{S}_{\mathrm{t}}\right)$ approaches zero if the market is contango, but $\left(\mathrm{S}_{\mathrm{t}}-\mathrm{F}_{\mathrm{t}}\right)$ approaches $+\infty$ if the market is in backwardation. 


\section{Diagram (2.3): The Matrix of Outcomes for an Offset Hedging}

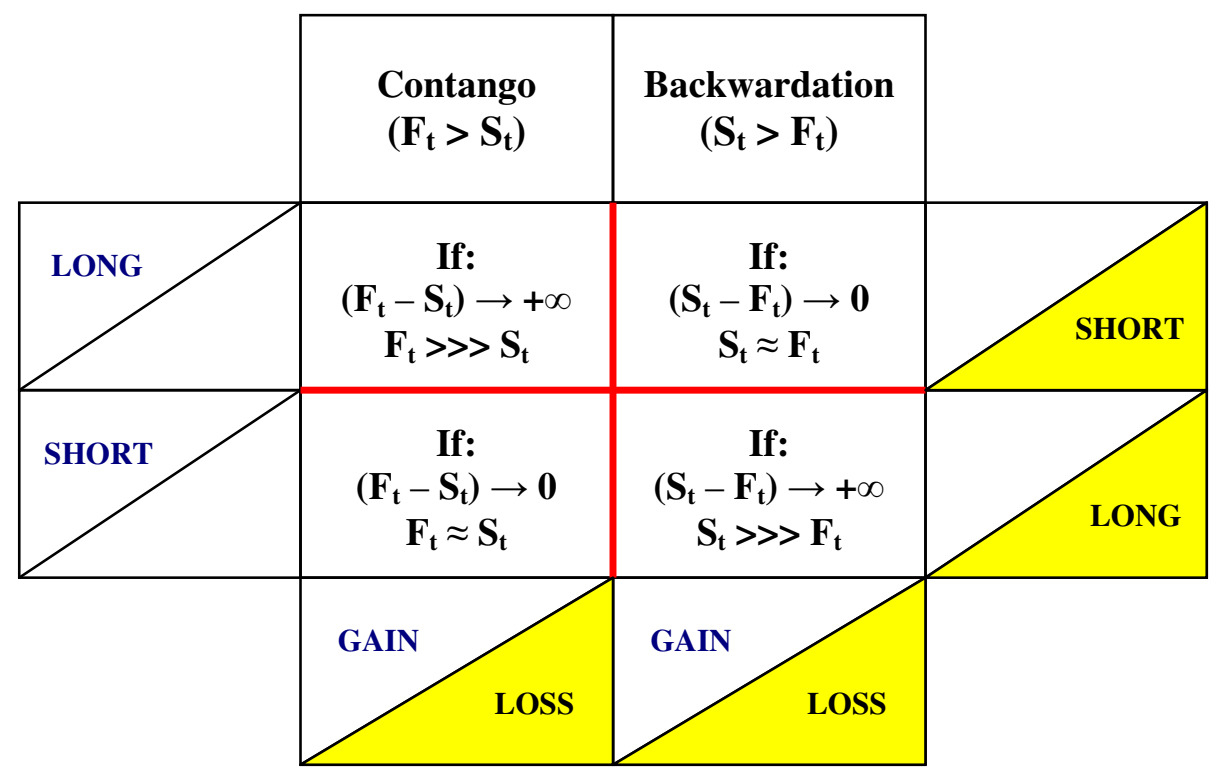

In a contango market, a long hedger will be hurt as $\left(\mathrm{F}_{\mathrm{t}}-\mathrm{S}_{\mathrm{t}}\right)$ approaches zero, and a short hedger will lose as $\left(\mathrm{F}_{\mathrm{t}}-\mathrm{S}_{\mathrm{t}}\right)$ approaches $+\infty$. If the market is in backwardation, a long hedger's loss increases as $\left(\mathrm{S}_{\mathrm{t}}-\mathrm{F}_{\mathrm{t}}\right)$ approaches $+\infty$, and a short hedger's loss increases as $\left(S_{t}-F_{t}\right)$ approaches zero.

In a price fix hedge, a long hedger will gain if $S_{T}>F_{0}$ and a short hedger will make a profit if $S_{T}<F_{0}$, where $F_{0}$ is a forward price at time zero that used to create an initial forward position and $\mathrm{S}_{\mathrm{T}}$ is the average cash settlement price of the delivery month. The long hedger (consumer) takes a long forward position at time zero at the forward price $\mathrm{F}_{0}$. When the contract matures at time $\mathrm{T}$, the long hedger closes his/her long forward position by selling back at the average cash settlement price $S_{\mathrm{T}}$ of the delivery month. Therefore, a long hedger's expected payoff from a price fix hedge is $\left(\mathrm{S}_{\mathrm{T}}-\mathrm{F}_{0}\right)$. In contrast, a short hedger's expected payoff from a price fix hedge is $\left(\mathrm{F}_{0}-\mathrm{S}_{\mathrm{T}}\right)$. 


\section{Diagram (2.4): The Matrix of Outcomes for a Price Fix Hedging}

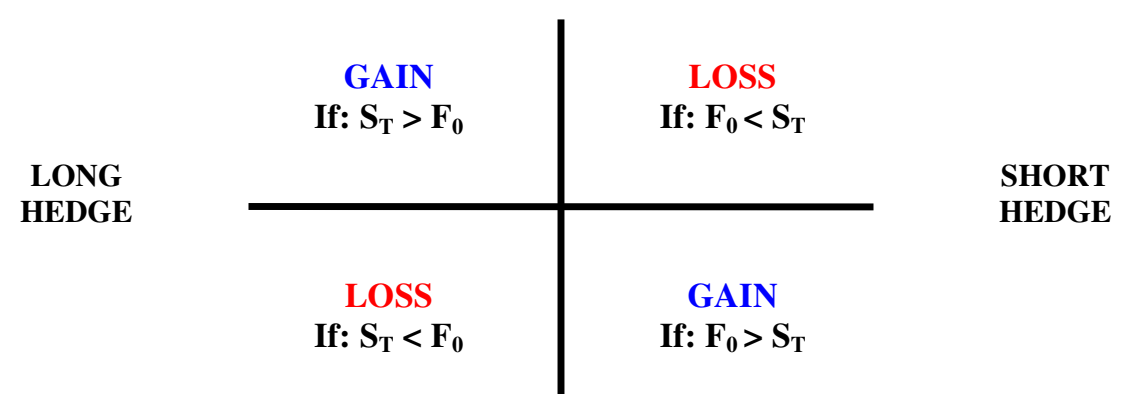

A short hedger's and a long hedger's actual and futures transactions are opposite because a short hedger's gain is said to be the long hedger's loss, and vise versa.

The cash and futures price movements have significant effects on variations in profits or losses of different positions in the spot and the futures market, so eight different cases of the spot and futures price movement and their outcomes are discussed here.

Notation: $\quad \mathrm{S}_{0}=$ spot price at time $0 \quad \mathrm{~F}_{0}=$ futures (forward) price at time 0

$$
\mathrm{S}_{\mathrm{t}}=\text { spot price at time } \mathrm{t} \quad \mathrm{F}_{\mathrm{t}}=\text { futures (forward) price at time } \mathrm{t}
$$

As we saw from table (2.9), the net effect of hedge is:

$$
\begin{gathered}
\circ\left\{\left(\mathrm{S}_{\mathrm{t}}-\mathrm{S}_{0}\right)+\left(\mathrm{F}_{0}-\mathrm{F}_{\mathrm{t}}\right)\right\} \text { for a short hedge } \\
\circ\left\{\left(\mathrm{S}_{0}-\mathrm{S}_{\mathrm{t}}\right)+\left(\mathrm{F}_{\mathrm{t}}-\mathrm{F}_{0}\right)\right\} \text { for a long hedge }
\end{gathered}
$$

\section{- Case 1: $\left\{S_{t}\right\}$ falls and $\left\{F_{t}\right\}$ falls by same amount as $\left\{S_{t}\right\}$}

a) A short hedge: If a trader is not hedged, the trader's long position in the spot market will result in loss of $\left(S_{t}-S_{0}\right)$ because $\left(S_{t}-S_{0}\right)<0$. If the trader is hedged, the net effect of the hedge will be zero because the trader's loss in the spot market equals the trader's gain in the futures market: $\left|S_{t}-S_{0}\right|=F_{0}-F_{t}>0$ 
b) A long hedge: If a trader is not hedged, the trader will profit by $\left(S_{0}-S_{t}\right)$ from his/her short spot position because $S_{0}>S_{t}$. If the trader is hedged, the net effect of the hedge will be zero because the trader's profit in the spot market will be entirely used to cover the losses in the futures market: $\left|F_{t}-F_{0}\right|=S_{0}-S_{t}>0$

\section{- Case 2: $\left\{S_{t}\right\}$ falls and $\left\{F_{t}\right\}$ falls by greater amount than $\left\{S_{t}\right\}$}

a) A short hedge: If not hedged, the trader will lose in the spot market. If hedged, the futures market gain will be greater than the spot market loss, so the trader's net effect of the hedge will be profitable: $\left|S_{t}-S_{0}\right|<F_{0}-F_{t}$

b) A long hedge: If not hedged, the trader will gain in the spot market. However, the gain in the spot market will be less than the futures market loss, if hedged. Therefore, the net effect of the hedge will not be profitable: $\left|F_{t}-F_{0}\right|>S_{0}-S_{t}$

\section{- Case 3: $\quad\left\{S_{t}\right\}$ falls and $\left\{F_{t}\right\}$ falls by smaller amount than $\left\{S_{t}\right\}$}

a) A short hedge: A long spot position will result in loss, if not hedged. If hedged, the net effect of the hedge will result in loss, but the loss will be smaller than the unhedged loss: $\left|S_{t}-S_{0}\right|>F_{0}-F_{t}$

b) A long hedge: A short spot position will result in profit, if not hedged. If hedged, the net effect of the hedge will be profitable, but the profit will be smaller than the unhedged profit: $\left|F_{t}-F_{0}\right|<S_{0}-S_{t}$

\section{- Case 4: $\left\{S_{t}\right\}$ falls and $\left\{F_{t}\right\}$ rises}

a) A short hedge: Without hedging, a long spot position will result in loss. If a trader is hedged, the net effect of the hedge will also not be profitable. Moreover, the loss will be greater than unhedged loss: $\left(S_{t}-S_{0}\right)<0,\left(F_{0}-F_{t}\right)<0$ 
b) A long hedge: Without hedging, a short spot position will result in profit. If hedged, the net effect of the hedge will also be profitable. Furthermore, the profit will be greater than unhedged profit: $\left(\mathrm{S}_{0}-\mathrm{S}_{\mathrm{t}}\right)>0,\left(\mathrm{~F}_{\mathrm{t}}-\mathrm{F}_{0}\right)>0$

\section{- Case 5: $\left\{S_{t}\right\}$ rises and $\left\{F_{t}\right\}$ rises by same amount as $\left\{S_{t}\right\}$}

a) A short hedge: Without hedging, a long spot position will be profitable because $\mathrm{S}_{0}<\mathrm{S}_{\mathrm{t}}$. If hedged, the net effect of the hedge will result in neither profit nor loss because the futures market loss equals the spot market gain: $\left|F_{0}-F_{t}\right|=S_{t}-S_{0}$

b) A long hedge: If not hedged, a short spot position will result in loss. If hedged, the net effect of the hedge will be zero because the loss in the spot market equals the gain in the futures market: $\left|S_{0}-S_{t}\right|=F_{t}-F_{0}$

\section{- Case 6: $\left\{S_{t}\right\}$ rises and $\left\{F_{t}\right\}$ rises by greater amount than $\left\{S_{t}\right\}$}

a) A short hedge: An unhedged long spot position will be profitable. If hedged though, the net effect of the hedge will result in loss because the gain in the spot market is less than the loss in the futures market: $\left|F_{0}-F_{t}\right|>S_{t}-S_{0}$

b) A long hedge: An unhedged short position in the spot market will result in loss. If hedged, the net effect of the hedge will be profitable because the futures market gain is greater than the spot market loss: $\left|S_{0}-S_{t}\right|<F_{t}-F_{0}$

\section{- Case 7: $\left\{S_{t}\right\}$ rises and $\left\{F_{t}\right\}$ rises by smaller amount than $\left\{S_{t}\right\}$}

a) A short hedge: The outcome of an unhedged long spot position is profitable. If hedged, the net effect of the hedge will also be profitable, but the profit will be smaller than unhedged profit: $\left|F_{0}-F_{t}\right|<S_{t}-S_{0}$ 
b) A long hedge: The outcome of an unhedged short spot position will result in loss. If hedged, the net effect of the hedge will not be profitable, but the loss will be smaller than an unhedged loss: $\left|S_{0}-S_{t}\right|>F_{t}-F_{0}$

\section{- Case 8: $\left\{S_{t}\right\}$ rises and $\left\{F_{t}\right\}$ falls}

a) A short hedge: An unhedged long spot position will result in gain. If hedged, the net effect of the hedge will be profitable. The profit will be greater than the unhedged profit because $\left(\mathrm{S}_{\mathrm{t}}-\mathrm{S}_{0}\right)>0,\left(\mathrm{~F}_{0}-\mathrm{F}_{\mathrm{t}}\right)>0$.

b) A long hedge: An unhedged short spot position will result in loss. If hedged, the net effect of the hedge will result in loss also. The loss will be greater than the unhedged loss because $\left(\mathrm{S}_{0}-\mathrm{S}_{\mathrm{t}}\right)<0,\left(\mathrm{~F}_{\mathrm{t}}-\mathrm{F}_{0}\right)<0$.

Table 2.10: Variations in Gains or Losses Resulting from Differences in Cash and Future Price Movements

\begin{tabular}{|c|c|c|c|c|c|}
\hline \multicolumn{2}{|c|}{ Price Movements } & \multicolumn{4}{|c|}{ Results } \\
\hline \multirow{2}{*}{$\begin{array}{l}\text { Spot } \\
\text { Price }\end{array}$} & \multirow{2}{*}{$\begin{array}{l}\text { Future } \\
\text { Price }\end{array}$} & \multicolumn{2}{|c|}{$\begin{array}{c}\text { Long in the spot market } \\
\text { (i.e. Short hedge) }\end{array}$} & \multicolumn{2}{|c|}{$\begin{array}{c}\text { Short in the spot market } \\
\text { (i.e. Long Hedge) }\end{array}$} \\
\hline & & Unhedged & Hedged & Unhedged & Hedged \\
\hline Falls & $\begin{array}{l}\text { Falls by same } \\
\text { amount as spot }\end{array}$ & Loss & $\begin{array}{c}\text { Neither profit } \\
\text { nor loss }\end{array}$ & Profit & $\begin{array}{l}\text { Neither profit } \\
\text { nor loss }\end{array}$ \\
\hline Falls & $\begin{array}{l}\text { Falls by greater } \\
\text { amount than spot }\end{array}$ & Loss & Profit & Profit & Loss \\
\hline Falls & $\begin{array}{l}\text { Falls by smaller } \\
\text { amount than spot }\end{array}$ & Loss & $\begin{array}{l}\text { Loss, but smaller } \\
\text { than unhedged loss }\end{array}$ & Profit & $\begin{array}{l}\text { Profit, but smaller } \\
\text { than unhedged profit }\end{array}$ \\
\hline Falls & Rises & Loss & $\begin{array}{l}\text { Loss, and greater } \\
\text { than unhedged loss }\end{array}$ & Profit & $\begin{array}{l}\text { Profit, and greater } \\
\text { than unhedged profit }\end{array}$ \\
\hline Rises & $\begin{array}{l}\text { Rises by same } \\
\text { amount as spot }\end{array}$ & Profit & $\begin{array}{c}\text { Neither profit } \\
\text { nor loss }\end{array}$ & Loss & $\begin{array}{c}\text { Neither profit } \\
\text { nor loss }\end{array}$ \\
\hline Rises & $\begin{array}{l}\text { Rises by greater } \\
\text { amount than spot }\end{array}$ & Profit & Loss & Loss & Profit \\
\hline Rises & $\begin{array}{l}\text { Rises by smaller } \\
\text { amount than spot }\end{array}$ & Profit & $\begin{array}{c}\text { Profit, but smaller } \\
\text { than unhedged } \\
\text { profit }\end{array}$ & Loss & $\begin{array}{l}\text { Loss, but smaller } \\
\text { than unhedged loss }\end{array}$ \\
\hline Rises & Falls & Profit & $\begin{array}{c}\text { Profit, and greater } \\
\text { than unhedged } \\
\text { profit }\end{array}$ & Loss & $\begin{array}{l}\text { Loss, and greater } \\
\text { than unhedged loss }\end{array}$ \\
\hline
\end{tabular}

Source: B. S. Yamey, “An Investigation of Hedging on an Organized Produce Exchange" Manchester School 19 (1951): 308 (Labys and Granger, 1970, p.24) 
The table above shows the variations of the gains or losses due to cash and futures price movements.

There are four types of option positions including long call, long put, short call and short put. The party agreeing to buy an option is said to have a long option position and the party agreeing to sell an option has a short option position. The following figures illustrate the payoffs of these positions graphically.

Notations:

$$
\begin{aligned}
& \mathrm{C}=\text { the value of a call option } \\
& \mathrm{P}=\text { the value of a put option } \\
& \mathrm{r}=\text { the risk free interest rate } \\
& \mathrm{e}=\text { exponential constant }
\end{aligned}
$$

Figure (2.2): Payoff from Long Call

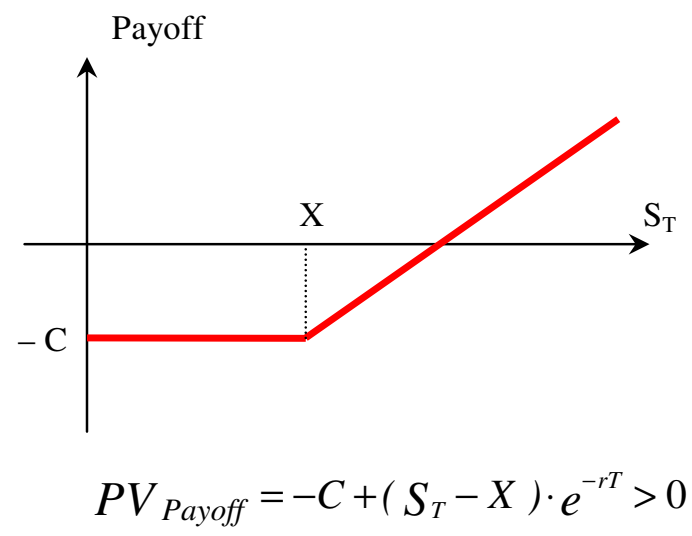

Figure (2.4): Payoff from Short Call

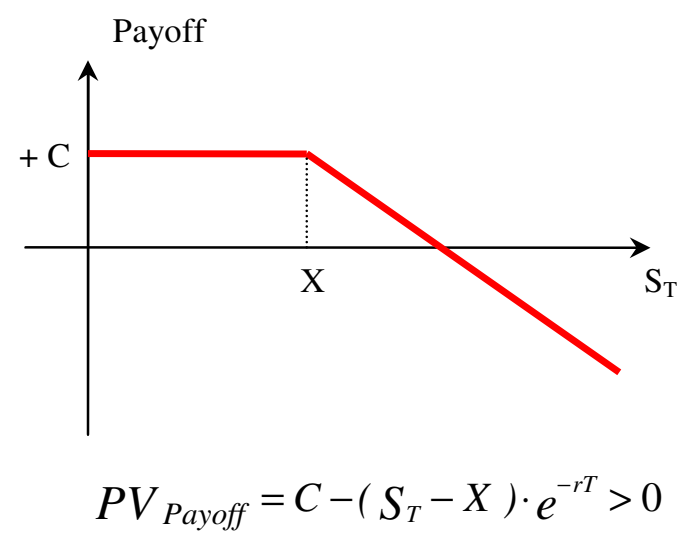

$\mathrm{S}_{\mathrm{T}}=$ the spot price at the maturity $\mathrm{T}$

$\mathrm{X}=$ the exercise price

$\mathrm{T}=$ the time to maturity

$\mathrm{PV}_{\text {Payoff }}=$ the present value of payoffs

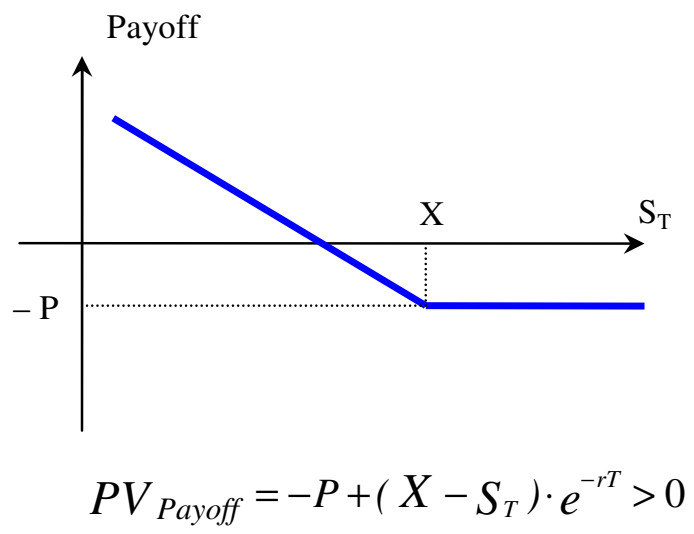

Figure (2.5): Payoff from Short Put

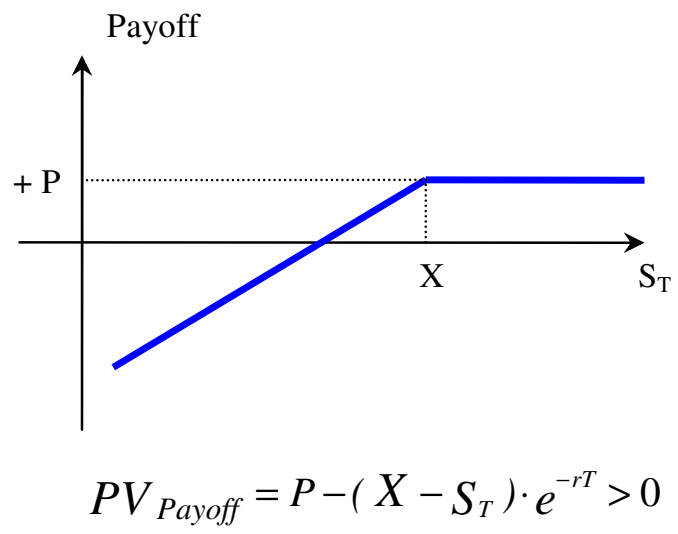


A long position in a call option has limited risk and unlimited reward because its payoffs at time zero and at the option's maturity are $\{-\mathrm{C}\}$ and the maximum of $\left\{\mathrm{S}_{\mathrm{T}}-\mathrm{X}\right.$, $0\}$ respectively. The risk of a long put option is also limited by the premium, but unlike a long call, its reward is limited by the exercise price because the option holder will receive the maximum of $\left\{0, X-S_{T}\right\}$ as payoff if the option is exercised. If a long call and a long put option are not exercised, the option holders will lose their premiums, but the writers of the options will be gained by those premiums. A call option writer (short position in a call option) receives the premium from an option holder at time zero, so the option writer's reward is limited by the premium. However, the option writer's risk is unlimited because the writer has to pay the option holder the maximum of $\left\{0, S_{T}-X\right\}$ as payoff if the option is exercised. Like a call option writer, a put option writer receives the premium from an option holder at time zero. Unlike a call writer, a put option writer's reward is limited by the exercise price because the writer has to pay the payoff of the maximum of $\left\{0, X-S_{T}\right\}$ if the option is exercised. Finally, the table below summarizes the risks and rewards from the basic futures and options positions.

Table 2.11: Summary of Risks and Rewards

\begin{tabular}{|l|c|l|c|c|}
\hline \multirow{2}{*}{ Position } & \multicolumn{2}{|c|}{ Risk } & \multicolumn{2}{c|}{ Reward } \\
\cline { 2 - 5 } & Payoff at $\mathrm{t}=0$ & & Payoff at $\mathrm{t}=\mathrm{T}$ & \\
\hline - Long Call & $\{-\mathrm{C}\}$ & Limited to prem. & $\operatorname{Max}\left\{0, \mathrm{~S}_{\mathrm{T}}-\mathrm{X}\right\}$ & Unlimited \\
\hline - Long Put & $\{-\mathrm{P}\}$ & Limited to prem. & $\operatorname{Max}\left\{0, \mathrm{X}-\mathrm{S}_{\mathrm{T}}\right\}$ & Limited by $\mathrm{X}$ \\
\hline - Short Call & $\{+\mathrm{C}\}$ & Unlimited & $-\operatorname{Max}\left\{0, \mathrm{~S}_{\mathrm{T}}-\mathrm{X}\right\}$ & Limited to prem. \\
\hline - Short Put & $\{+\mathrm{P}\}$ & Unlimited & $-\operatorname{Max}\left\{0, \mathrm{X}-\mathrm{S}_{\mathrm{T}}\right\}$ & Limited by $\{-\mathrm{X}\}$ \\
\hline - Long Future & $\mathrm{IF:}\left(\mathrm{F}_{\mathrm{t}}-\mathrm{S}_{\mathrm{t}}\right)-\left(\mathrm{F}_{0}-\mathrm{S}_{0}\right)<0$ & IF: $\left(\mathrm{F}_{\mathrm{t}}-\mathrm{S}_{\mathrm{t}}\right)-\left(\mathrm{F}_{0}-\mathrm{S}_{0}\right)>0$ \\
& \multicolumn{2}{|c|}{ Almost unlimited } & Unlimited \\
\hline - Short Future & IF: $\left(\mathrm{F}_{0}-\mathrm{S}_{0}\right)-\left(\mathrm{F}_{\mathrm{t}}-\mathrm{S}_{\mathrm{t}}\right)<0$ & IF: $\left(\mathrm{F}_{0}-\mathrm{S}_{0}\right)-\left(\mathrm{F}_{\mathrm{t}}-\mathrm{S}_{\mathrm{t}}\right)>0$ \\
\hline
\end{tabular}




\section{CHAPTER III:}

\section{VALUING COMMODITY DERIVATIVES}

In this chapter, theoretical valuation issues of futures and options will be discussed because it is important to OTC derivatives' traders to understand how theoretical (fair) prices of the options and forward contracts are valued. The focuses of the analysis in this chapter are: first, to calculate implied volatilities of the underlying commodity prices; second, to value Asian options on different dates and third, to compare theoretically determined Asian option values to their observed market values.

\subsection{FAIR VALUES OF FUTURES (FORWARD) CONTRACTS}

Assume that the metal is only to be used in the future.

Any consumer has two options about purchasing a metal. First, purchasing the physical metal immediately, and alternatively, purchasing the metal in the future. In the first case, the consumer will finance his/her purchase by borrowing or withdrawing the money from a bank account because it is an immediate purchase. If the consumer borrows the money, there will be an interest rate penalty, which means the loan must be paid with its interest. If the consumer withdraws the money, there will be a loss of interest on his/her credit balances. Additionally, the consumer must pay for insurance and storage costs. In the second case, the consumer will only pay for the metal in the future; hence, he/she is able to save on insurance and storage costs. 
Therefore, the futures price incorporates the costs of finance, insurance and storage, which is known as the carrying charges. The minimum acceptable price (fair value) of a forward contract today is, therefore, the cash price plus the carrying charges.

The cost of carry model is used for pricing futures/forward contracts.

$$
\begin{aligned}
& \text { Fair value } \\
& \text { of } \\
& \text { Futures contract }
\end{aligned}=\text { Cash Price }+ \text { Carrying Charges }
$$

If storage charges are given in percentage terms, a fair value of commodity futures will be defined as follows:

$$
F_{0}=S_{0} \cdot\left[1+\left(C_{I}+C_{S}\right) \cdot \frac{T}{360}\right]
$$

Where, $\quad \mathrm{F}_{0}=$ Futures price $\quad \mathrm{S}_{0}=$ Spot price $\quad \mathrm{T}=$ maturity (by days)

$$
\mathrm{C}_{\mathrm{I}}=\text { Interest }(\% \text { per year }) \quad \mathrm{C}_{\mathrm{S}}=\text { Storage charges }(\% \text { per year })
$$

The equation above can be written differently, depending on how interest rates and storage costs are expressed. For instance, in the situation of a continuously compounded interest rate, the equation below is useful.

$$
\begin{aligned}
& \qquad F_{0}=\left(S_{0}+U\right) e^{r T} \\
& \mathrm{r}=\text { the risk free interest rate (\% per year) } \quad \mathrm{T}=\text { time to maturity (by years) } \\
& \mathrm{e}=\text { exponential constant } \\
& \mathrm{U}=\text { the present value of all the storage costs }
\end{aligned}
$$


If the storage costs incurred at any time are proportional to the price of the underlying commodity, they can be regarded as providing a negative yield (Hull, 2002, p.60). In this case, the equation (3.3) will be converted to the equation below.

$$
F_{0}=S_{0} e^{(r+u) T}
$$

Where, $\mathrm{u}=$ the storage costs per annum as a proportion of the spot price

A consumption commodity holder gets a benefit, which is called convenience yield (the market's expectations concerning the future availability of the commodity) if the holder actually owns a physical commodity. The purposes of holding an inventory of a consumption commodity include consumption, production input, transaction and precautionary motives, and taking advantage of temporary shortages of the material (Johnson, 2004, p.9). Copper is a consumption commodity, so the convenience yield should be considered for pricing forward contracts on copper. If the convenience yield that is provided by the commodity is noted $\mathrm{y}$ as a per annum continuous yield, then equations (3.3) and (3.4) become:

$$
\begin{gathered}
F_{0} e^{y T}=\left(S_{0}+U\right) e^{r T} \\
F_{0} e^{y T}=S_{0} e^{(r+u) T} \quad \text { or } \quad F_{0}=S_{0} e^{(r+u-y) T}
\end{gathered}
$$

Replication and arbitrage table (3.1) shows derivation of equation (3.6).

In the spot market, a commodity holder gets a convenience benefit as a positive yield because the holder has a long spot position. However, the storage costs can be regarded as providing a negative yield. Therefore, the commodity holder's payoff at time zero will be $\left\{-e^{(u-y) T} \cdot S_{0}\right\}$. The payoff at time $\mathrm{T}$ will be $\mathrm{S}_{\mathrm{T}}$ in the spot market. 
Table 3.1: Cost of Carry Formula with Convenience Yields

\begin{tabular}{|c|c|c|}
\hline Spot & Time 0 & Time T \\
\hline - Buy the asset & $-e^{(u-y) T} \cdot S_{0}$ & $S_{T}$ \\
\hline Forward & Time 0 & Time T \\
\hline - Long forward & & $S_{T}-F_{0}$ \\
\hline - Lend $F_{0} \cdot e^{-r T}$ & $-F_{0} \cdot e^{-r T}$ & $F_{0}$ \\
\hline Total & $-F_{0} \cdot e^{-r T}$ & $S_{T}$ \\
\hline
\end{tabular}

(Source: Johnson, 2004, p. 9)

In the futures market, the payoff from a long forward position at time $T$ is $S_{T}-F_{0}$. The payoff at time $\mathrm{T}$ from the invested money $-F_{0} \cdot e^{-r T}$ will be $\mathrm{F}_{0}$. Hence, the payoff at time $T$ in the futures market will be: $\left(S_{T}-F_{0}\right)+F_{0}=S_{T}$, which equals the payoff at time $\mathrm{T}$ in the spot market. Therefore, the payoffs at time zero in the futures and spot markets should be the same, so the following equation is true:

$$
\begin{aligned}
-F_{0} \cdot e^{-r T} & =-e^{(u-y) T} \cdot S_{0} \\
F_{0} & =S_{0} e^{(r+u-y) T}
\end{aligned}
$$

Where: the storage costs $(\mathrm{u})$ and convenience yield (y) per annum are as proportions of the spot price.

If the convenience yield is known, then equation (3.6) is useful, otherwise equation (3.3) is useful. The convenience benefit is known when the commodity is bought. 


\subsection{VALUING ASIAN OPTIONS}

As we mentioned before, the Over-the-Counter (OTC) market is an informal market without a centralized exchange or trading floor. On the OTC market, derivative products such as forwards, swaps and exotic options are traded between financial institutions and their corporate clients. These derivatives are not standardized, so trading the OTC derivatives give more flexibility to corporate clients, rather than the standard contracts such as futures contracts, European options and American options.

American option is an option that can be exercised at any time up to and including the expiration date of the option; whereas, European option is an option that can be exercised only when it expires. American and European options are commonly traded on various types of financial instruments such as stocks, bonds, currencies, interest rates, stock indices, commodities on the exchanges because they are standardized.

Both European and Asian style options and futures contracts on copper are available on the LME, but American style options are not traded on the LME. Hence, only Asian and European options can be traded on base metals of the LME. For the LME options, the maturity of European style options is the morning of the first Wednesday of the month. If exercised, they result in a third Wednesday of the month position. European options on the LME are manually exercised.

An Asian style option is one of the exotic options that is commonly traded in the OTC market. These types of options are less expensive than regular (standard) options, so Asian options are more appropriate than standard options for corporate customers. The main focus of this research is in the OTC market; therefore, only Asian options are valued in this chapter. 
Asian options, which are traded on the LME are also known as TAPO. The acronym TAPO stands for Traded Average Price Option. These options are available for the base metals. The payoffs of an Asian option depend on the history of the random walk of the asset price via some sort of average. Hence, the final payoff of an Asian option is determined by the average price of the asset during the option's life.

The expected payoffs from an average price call and an average price put option are the maximum of $\left\{0, S_{A V G(t)}-X\right\}$ and the maximum of $\left\{0, X-S_{A V G(t)}\right\}$ respectively, where $S_{A V G(t)}$ is the average value of the underlying asset calculated over a predetermined averaging period (usually a month) and $\mathrm{X}$ is the exercise price (Hull, 2000, p.467).

As we discussed in the previous chapter, there are four different positions in options. The payoffs of Asian call and put options positions are:

* Long Position in an Asian Call:

$P V_{\text {Payoff }}=-C+\left(S_{A V G(t)}-X\right) \cdot e^{-r T}>0$

$\star$ Short Position in an Asian Call:

$P V_{\text {Payoff }}=C-\left(S_{A V G(t)}-X\right) \cdot e^{-r T}>0$
* Long Position in an Asian Put:

$$
P V_{\text {Payoff }}=-P+\left(X-S_{A V G(t)}\right) \cdot e^{-r T}>0
$$

$\star$ Short Position in an Asian Put:

$$
P V_{\text {Payoff }}=P-\left(X-S_{A V G(t)}\right) \cdot e^{-r T}>0
$$

Where, $\quad \mathrm{C}=$ the value of an Asian call option $\quad \mathrm{P}=$ the value of an Asian put option $\mathrm{S}_{\mathrm{AVG}(\mathrm{t})}=$ the average cash settlement price in the calculation period $\mathrm{t}$

$$
\begin{array}{ll}
\mathrm{X}=\text { the exercise price } & \mathrm{r}=\text { the risk free interest rate } \\
\mathrm{T}=\text { the time to maturity } & \mathrm{e}=\text { exponential constant } \\
\mathrm{t}=\text { the predetermined averaging period } & \mathrm{PV}_{\text {Payoff }}=\text { the present value of payoffs }
\end{array}
$$


Moreover, Asian style options on the LME are automatically exercised and settled against the average of the reference price over the calculation period, which is most commonly the calendar month on the LME. Therefore, the option expires over the course of the month, ending on the last trading day with financial settlement typically on the second business day of the following month. On the LME, the reference price is almost always the month average of the LME cash settlement price.

The value of an option consists of its intrinsic (terminal) value and the time value of the option. Options, which have intrinsic value, are described as being in-the-money, but options which have only time value are described as being out-of-the-money. Options are more expensive the longer the remaining life until the expiration date because writers of the options want larger rewards for taking larger risks until the maturity. The time value of an option will be low when the option is deep in-the-money because it is inevitable that the option will be exercised and deep out-of-the-money because it is inevitable that the option will not be exercised. By contrast, the time value will be greatest for options, which are at-the-money because there is a 0.5 and 0.5 chances of the option being exercised, so all risks and uncertainties are concentrated in the at-the-money option. Therefore, time value helps options' writers, but hurts options' holders.

There are two basic models used for pricing options: Binomial and Black-Scholes models. The best known of options pricing models is the Nobel Prize winner ('97) BlackScholes model. The formulas that define the Black-Scholes options' pricing model are:

$$
\left\{\begin{array}{l}
C=S \cdot N\left(d_{1}\right)-X e^{-r T} \cdot N\left(d_{2}\right) \\
P=X e^{-r T} \cdot N\left(-d_{2}\right)-S \cdot N\left(-d_{1}\right)
\end{array}\right.
$$




$$
d_{1}=\frac{\operatorname{Ln}\left(\frac{S}{X}\right)+\left(r+\frac{\sigma^{2}}{2}\right) T}{\sigma \sqrt{T}} \text { and } d_{2}=\frac{\operatorname{Ln}\left(\frac{S}{X}\right)+\left(r-\frac{\sigma^{2}}{2}\right) T}{\sigma \sqrt{T}}=d_{1}-\sigma \sqrt{T}
$$

where,

$\mathrm{C}=$ the value of a call option $\quad \mathrm{P}=$ the value of a put option

$\mathrm{S}=$ the current asset price $\quad \mathrm{X}=$ the exercise price

$\mathrm{r}=$ the risk-free interest rate $\quad \mathrm{T}=$ the time to expiration (in years)

e = exponential constant $\quad \mathrm{N}\left(\mathrm{d}_{1}\right)$ and $\mathrm{N}\left(\mathrm{d}_{2}\right)=$ the normal probability distributions

$\sigma=$ the volatility of the asset $(\%$ per year)

The determinants of an option value include the underlying asset price, the strike price, the time to maturity, the asset's price volatility, and the interest rate. The most influential factor on an option's value is the volatility, which is a measure of how much an underlying price varies. There is a positive relationship between the volatility and the value of an option for both calls and puts because the risk for the options' writer becomes large if an underlying asset varies a great deal. Accordingly, the options' writers demand larger premiums, so the value of the option rises.

Based upon the Black-Scholes model, Asian options can be valued using the Derivative Gem (DerivaGem) software package. The software was developed by A-J, Financial Systems Inc. In order to calculate theoretical option prices using DerivaGem, the input variables of the options prices must be calculated. These are discussed next. 


\section{A) The Risk-Free Interest Rate:}

The appropriate risk free interest rate in the options' valuation is determined based on the U.S. T-bill discount rates with constant maturities. The T-bill rate is less risky than the LIBOR rate, so it is a useful and appropriate rate for defining the risk-free rate in the options pricing model. The formula below defines the appropriate risk free interest rate, which is one of the input variables of the pricing model.

$$
\mathrm{r}=-\frac{\operatorname{Ln}\left[1-0.01 \cdot \mathrm{d} \cdot \frac{\mathrm{T}}{360}\right]}{\mathrm{t}}=-\frac{\operatorname{Ln}\left[1-0.01 \cdot \mathrm{d} \cdot \frac{\mathrm{T}}{360}\right]}{\frac{\mathrm{T}}{365}}
$$

$$
\begin{aligned}
& \text { where, } \quad \mathrm{r}=\text { the risk-free interest rate } \quad \mathrm{d}=\text { discount yield (T-Bill rate) } \\
& \mathrm{T}=\text { maturity of the bill (in days) Source: (Johnson, 2004, p. 65) }
\end{aligned}
$$

\section{B) Historical Volatility:}

What traders, hedgers and risk managers want to know is how volatile an asset will be in the future. Historical volatility is determined based upon the historical price series, so it can be useful for pricing options. There is a rule of thumb: to set $n$ equal to the number of days (months) to which the volatility is to be applied. If the volatility is used to value 36-month options, 36 months of historical data are used.

$$
\begin{array}{ll}
\mathrm{U}=\text { Monthly return: } & U_{i}=\ln \left(\frac{S_{i}}{S_{i-1}}\right) \\
\mathrm{SD}=\text { the standard deviation of the } \mathrm{U}_{\mathrm{i}} \text { 's: } & S D=\sqrt{\frac{1}{n-1} \sum_{i=1}^{n} U_{i}^{2}-\frac{1}{n(n-1)}\left(\sum_{i=1}^{n} U_{i}\right)^{2}}
\end{array}
$$




$$
\sigma_{\text {Historical }}=\frac{S D}{\sqrt{\tau}}
$$

Where,

$\mathrm{S}_{\mathrm{i}}=$ the asset price at the end of ith interval; $\quad \tau=$ length of time interval in years;

$\mathrm{SE}=$ the standard error of historical volatility estimate; $\mathrm{i}=$ months

$\sigma_{\text {Historical }}=$ historical volatility

\section{C) Implied Volatility:}

Implied volatilities of the underlying asset price are calculated by using a pricing model. The inputs are the spot price of an underlying asset (S), the exercise price $(\mathrm{X})$, the time to maturity $(\mathrm{T})$, the interest rates $(\mathrm{r})$, the averaging period $(\mathrm{t})$ and the actual market value of the option $(\mathrm{C}$ or $\mathrm{P})$. Using the implied volatility may be better than relying on historical volatilities, which may not fully incorporate the impact of information recently released to the market. Therefore, instead of using historical volatilities, calculating implied volatilities and using them for pricing options are essential. The implied volatility estimates are calculated in data analysis section (3.4).

\subsection{DATA DESCRIPTION AND COLLECTION}

The data used for valuing options were collected from OTC option's quotes on different dates, in which some options had already expired and the others are expected to mature. The expired options were collected from the OTC weekly quotes starting from 01 August 2002 to 05 September 2002. The options, which are expected to mature, were collected from the $21^{\text {st }}$ and $22^{\text {nd }}$ of February 2005. 
Table 3.2: The LME Option Premiums (US\$/t) for Copper

\begin{tabular}{|c|c|c|c|c|c|}
\hline \multirow{2}{*}{$\begin{array}{l}\text { Value } \\
\text { Date }\end{array}$} & \multirow{2}{*}{$\begin{array}{c}\text { Expiry } \\
\text { date }\end{array}$} & \multicolumn{2}{|c|}{ Put option } & \multicolumn{2}{|c|}{ Call option } \\
\hline & & Strike & Premiums & Strike & Premiums \\
\hline \multirow{6}{*}{ 01-Aug-02 } & \multirow{3}{*}{$4 q-02$} & 1525 & 67 & 1525 & 43 \\
\hline & & 1475 & 42 & 1575 & 26 \\
\hline & & 1450 & 32 & 1600 & 20 \\
\hline & \multirow{3}{*}{$2 q-03$} & 1525 & 86 & 1525 & 94 \\
\hline & & 1475 & 63 & 1575 & 72 \\
\hline & & 1450 & 53 & 1600 & 63 \\
\hline \multirow{6}{*}{ 08-Aug-02 } & \multirow{3}{*}{$4 q-02$} & 1525 & 73 & 1525 & 36 \\
\hline & & 1475 & 45 & 1575 & 21 \\
\hline & & 1450 & 34 & 1600 & 16 \\
\hline & \multirow{3}{*}{$2 q-03$} & 1525 & 91 & 1525 & 86 \\
\hline & & 1475 & 66 & 1575 & 65 \\
\hline & & 1450 & 56 & 1600 & 56 \\
\hline \multirow{6}{*}{ 15-Aug-02 } & \multirow{3}{*}{$4 q-02$} & 1525 & 66 & 1525 & 36 \\
\hline & & 1475 & 39 & 1575 & 20 \\
\hline & & 1450 & 29 & 1600 & 15 \\
\hline & \multirow{3}{*}{$2 q-03$} & 1525 & 85 & 1525 & 87 \\
\hline & & 1475 & 62 & 1575 & 66 \\
\hline & & 1450 & 51 & 1600 & 57 \\
\hline \multirow{6}{*}{ 22-Aug-02 } & \multirow{3}{*}{$4 q-02$} & 1525 & 59 & 1525 & 37 \\
\hline & & 1475 & 34 & 1575 & 21 \\
\hline & & 1450 & 24 & 1600 & 15 \\
\hline & \multirow{3}{*}{$2 q-03$} & 1525 & 80 & 1525 & 90 \\
\hline & & 1475 & 58 & 1575 & 68 \\
\hline & & 1450 & 48 & 1600 & 59 \\
\hline \multirow{6}{*}{ 30-Aug-02 } & \multirow{3}{*}{$4 q-02$} & 1525 & 50 & 1525 & 40 \\
\hline & & 1475 & 27 & 1575 & 22 \\
\hline & & 1450 & 19 & 1600 & 16 \\
\hline & \multirow{3}{*}{$2 q-03$} & 1525 & 75 & 1525 & 97 \\
\hline & & 1475 & 53 & 1575 & 74 \\
\hline & & 1450 & 44 & 1600 & 64 \\
\hline \multirow{6}{*}{ 05-Sep-02 } & \multirow{3}{*}{$4 q-02$} & 1525 & 71 & 1525 & 22 \\
\hline & & 1475 & 41 & 1575 & 11 \\
\hline & & 1450 & 29 & 1600 & 7 \\
\hline & \multirow{3}{*}{$2 q-03$} & 1525 & 92 & 1525 & 75 \\
\hline & & 1475 & 67 & 1575 & 55 \\
\hline & & 1450 & 56 & 1600 & 47 \\
\hline \multirow{6}{*}{ 12-Sep-02 } & \multirow{3}{*}{$4 q-02$} & 1550 & 54 & 1550 & 32 \\
\hline & & 1500 & 29 & 1575 & 23 \\
\hline & & 1450 & 14 & 1625 & 12 \\
\hline & & 1550 & 80 & 1550 & 89 \\
\hline & $2 q-03$ & 1500 & 57 & 1575 & 77 \\
\hline & & 1450 & 39 & 1625 & 58 \\
\hline
\end{tabular}

(Source: Ahmed [2002], Credit Lyonnais Rouse Limited) 
Category-1 members (ring dealing members) of the LME including Sempra Metals Group and Credit Lyonnais Rouse Limited (Calyon Financial) provided average price options' (Asian) quotes on the LME. Both call and put options with different maturities and different exercise prices are available for these options' quotes. The OTC weekly option quotes with different types, exercises prices and maturities are shown in tables (3.2) and (3.3). These indications are monthly settled Asian options that exercised against the average cash settlement price of the maturity month. In other words, July 2005 through December 2005 is for six monthly settled options, not one option over 6 months.

Table 3.3: The LME Option Premiums (US\$/t) for Copper

\begin{tabular}{|c|c|c|c|c|c|c|c|}
\hline 21-Feb-05 & \multicolumn{7}{|c|}{ PUT OPTION PREMIUMS } \\
\hline \multirow[b]{2}{*}{ Period (Maturity) } & \multirow[b]{2}{*}{ SWAP } & \multicolumn{6}{|c|}{ STRIKE } \\
\hline & & 2200 & 2300 & 2400 & 2500 & 2600 & 2700 \\
\hline July 2005 - Dec 2005 & 3027 & 12.46 & 20.52 & 30.19 & 45.75 & 63.55 & 89.82 \\
\hline Jan 2006 - Dec 2006 & 2790 & 58.87 & 81.54 & 104.74 & 137.61 & 170.62 & 214.64 \\
\hline Jan 2007 - Dec 2007 & 2609 & 105.27 & 137.48 & 168.40 & 210.81 & 258.49 & 311.20 \\
\hline 22-Feb-05 & \multicolumn{7}{|c|}{ PUT OPTION PREMIUMS } \\
\hline \multirow[b]{2}{*}{ Period (Maturity) } & \multirow[b]{2}{*}{ SWAP } & \multicolumn{6}{|c|}{ STRIKE } \\
\hline & & 2200 & 2300 & 2400 & 2500 & 2600 & 2700 \\
\hline July 2005 - Dec 2005 & 3058 & 11.29 & 18.70 & 27.61 & 42.07 & 58.63 & 83.31 \\
\hline Jan 2006 - Dec 2006 & 2815 & 55.50 & 77.17 & 99.35 & 130.99 & 162.76 & 205.42 \\
\hline Jan 2007 - Dec 2007 & 2630 & 101.05 & 132.34 & 162.34 & 203.75 & 250.43 & 302.15 \\
\hline
\end{tabular}

\begin{tabular}{|c|c|c|c|c|c|c|c|}
\hline \multirow[b]{3}{*}{ Period (Maturity) } & \multicolumn{7}{|c|}{ CALL OPTION PREMIUMS } \\
\hline & \multirow[b]{2}{*}{ SWAP } & \multicolumn{6}{|c|}{ STRIKE } \\
\hline & & 3100 & 3200 & 3300 & 3400 & 3500 & 3600 \\
\hline July 2005 - Dec 2005 & 3027 & 192.56 & 154.87 & 123.27 & 97.17 & 75.89 & 58.77 \\
\hline Jan 2006 - Dec 2006 & 2790 & 151.14 & 125.34 & 103.42 & 84.95 & 69.47 & 56.59 \\
\hline Jan 2007 - Dec 2007 & 2609 & 117.13 & 98.12 & 81.95 & 68.24 & 56.68 & 46.96 \\
\hline
\end{tabular}

\begin{tabular}{|c|c|c|c|c|c|c|c|}
\hline \multirow[b]{3}{*}{ Period (Maturity) } & \multicolumn{7}{|c|}{ CALL OPTION PREMIUMS } \\
\hline & \multirow[b]{2}{*}{ SWAP } & \multicolumn{6}{|c|}{ STRIKE } \\
\hline & & 3100 & 3200 & 3300 & 3400 & 3500 & 3600 \\
\hline July 2005 - Dec 2005 & 3058 & 208.17 & 168.40 & 134.84 & 106.92 & 83.99 & 65.42 \\
\hline Jan 2006 - Dec 2006 & 2815 & 159.99 & 133.05 & 110.09 & 90.67 & 74.35 & 60.72 \\
\hline Jan 2007 - Dec 2007 & 2630 & 122.86 & 103.13 & 86.30 & 72.00 & 59.92 & 49.74 \\
\hline
\end{tabular}

(Source: Ziegelaar [2005], Sempra Metals Group) 
Similarly, January 2006 through December 2006 is for twelve monthly settled options, not one option over 12 months. For example, a copper producer could buy an Asian put option with the exercise price of $\$ 2,700 / t$ and the maturity date of January through December 2006 at the price of $\$ 214.64 / t$ on 21 February 2005. If the maturity date was December-06, then the averaging period ( $\mathrm{t}$ ) would be the month of December06. In other words, the option would be exercised against the average cash settlement price of December-06.

Put options are more expensive at higher strikes, but call options are more expensive at lower strikes. In addition, these quotes contain SWAP values over a specific year. The procedure of metal swaps contracts are basically similar to the procedure of price fix hedges. The metal swaps consist of monthly settled forward contracts. On 21 February 2005 , a copper producer could sell copper at the swap price $\$ 3,027 /$ in July through December 2005, at $\$ 2,790 / \mathrm{t}$ in January through December 2006, and at $\$ 2,609 / \mathrm{t}$ in January through December 2007.

In table (3.2), the option prices of the first week will be used for calculating implied volatilities. For instance, the data from the previous date (01-August-02) were used to determine implied volatilities for several different options with different exercise prices, expiration dates and types (calls and puts) in order to value the options on the next date, one week after, for 08 August 02 . This procedure continues through all the quotes.

In table (3.3), the previous day's quotes ( $21^{\text {st }}$ of February) of these two consecutive days' data were used for calculating implied volatilities in order to value the options on the next day of $22^{\text {nd }}$ of February. All implied volatility calculations are in the data analysis section. 
The official web page of the Federal Reserves reports Treasury bill discount rates with constant maturities on its statistical release. The official web page of the LME reports daily cash settlement prices of A-grade copper.

The predetermined averaging period $(\mathrm{t})$ is expressed as a time since inception in DerivaGem software and it always equals 30 days (0.0822 years) because the Asian options on the LME base metals are settled against the average cash settlement prices of the maturity month.

The maturity date is the second business day of the following month of an option's expiration month. For instance, July-05 option matures on 2 August-05, which is the second business day of August-05. The analysis and options valuations are in the following section.

\subsection{DATA ANALYSIS}

The following figures show the implied volatility (I.V.) estimations on different dates as that specified. The details of implied volatility estimation are illustrated in appendix 1 .

Notations: $\quad$ I.V. $=$ the implied volatility $(\%$ per year $)$

$$
\mathrm{T}=\text { the maturity date } \quad \mathrm{X}=\text { the exercise price }
$$

OTM = out-of-the-money $\quad$ ITM = in-the-money

ATM = at-the-money 
Figure (3.1): Implied Vol. (Call, 01/08/02)

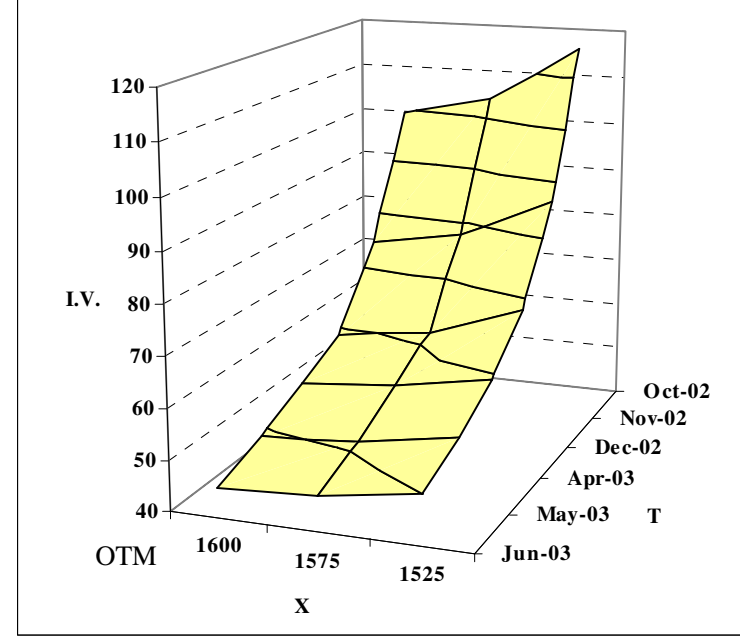

Figure (3.2): Implied Vol. (Call, 08/08/02)

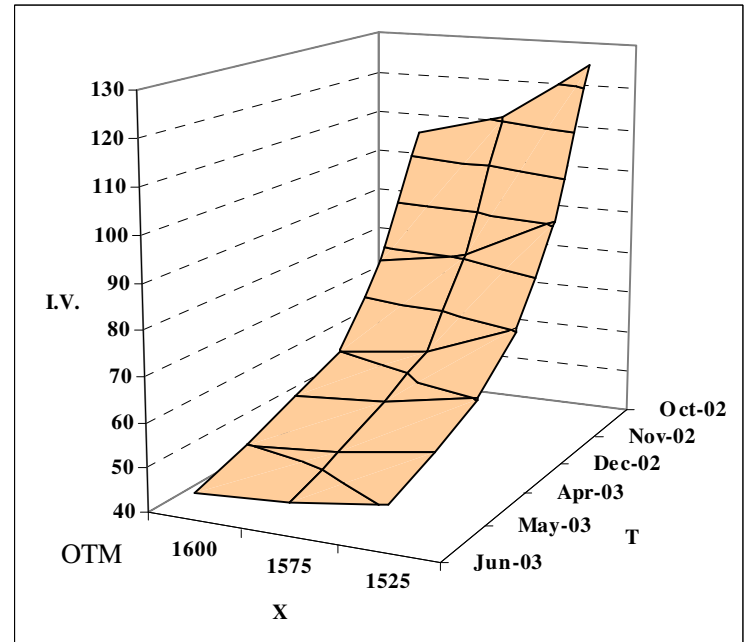

Figure (3.3): Implied Vol. (Call, 15/08/02)

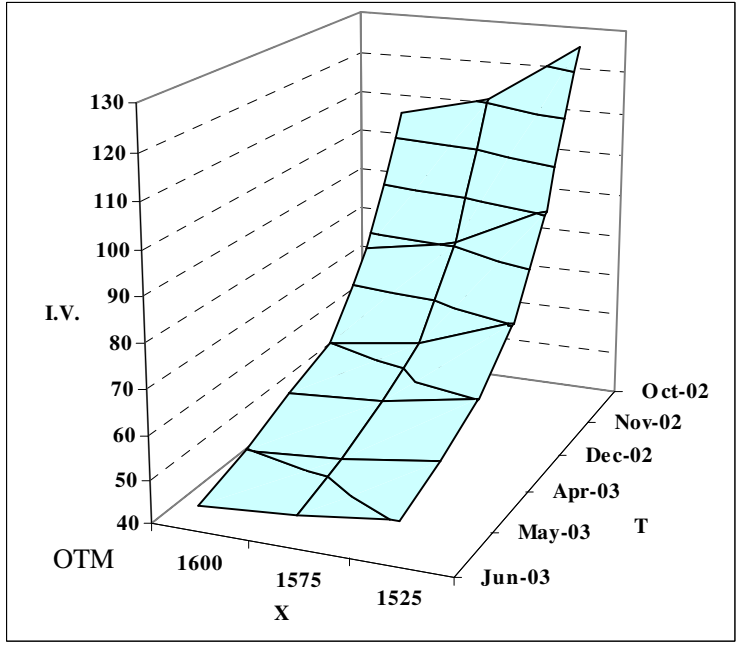

Figure (3.4): Implied Vol. (Call, 22/08/02)

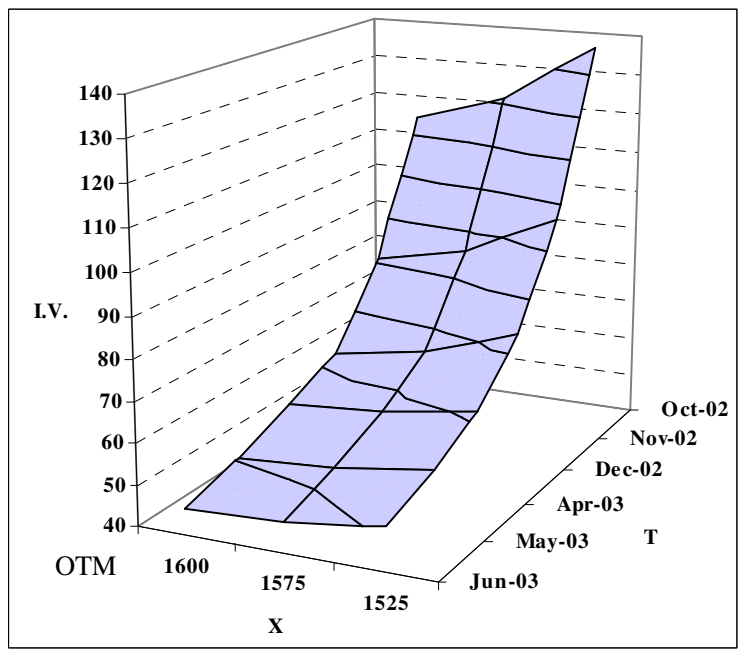

Figure (3.5): Implied Vol. (Call, 30/08/02)

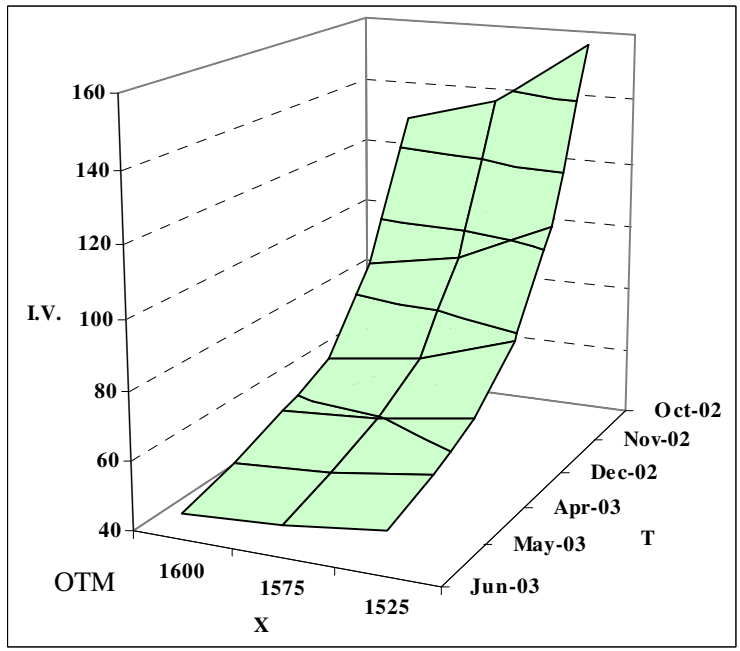


Figure (3.6): Implied Volatilities (Call, 21/02/05)

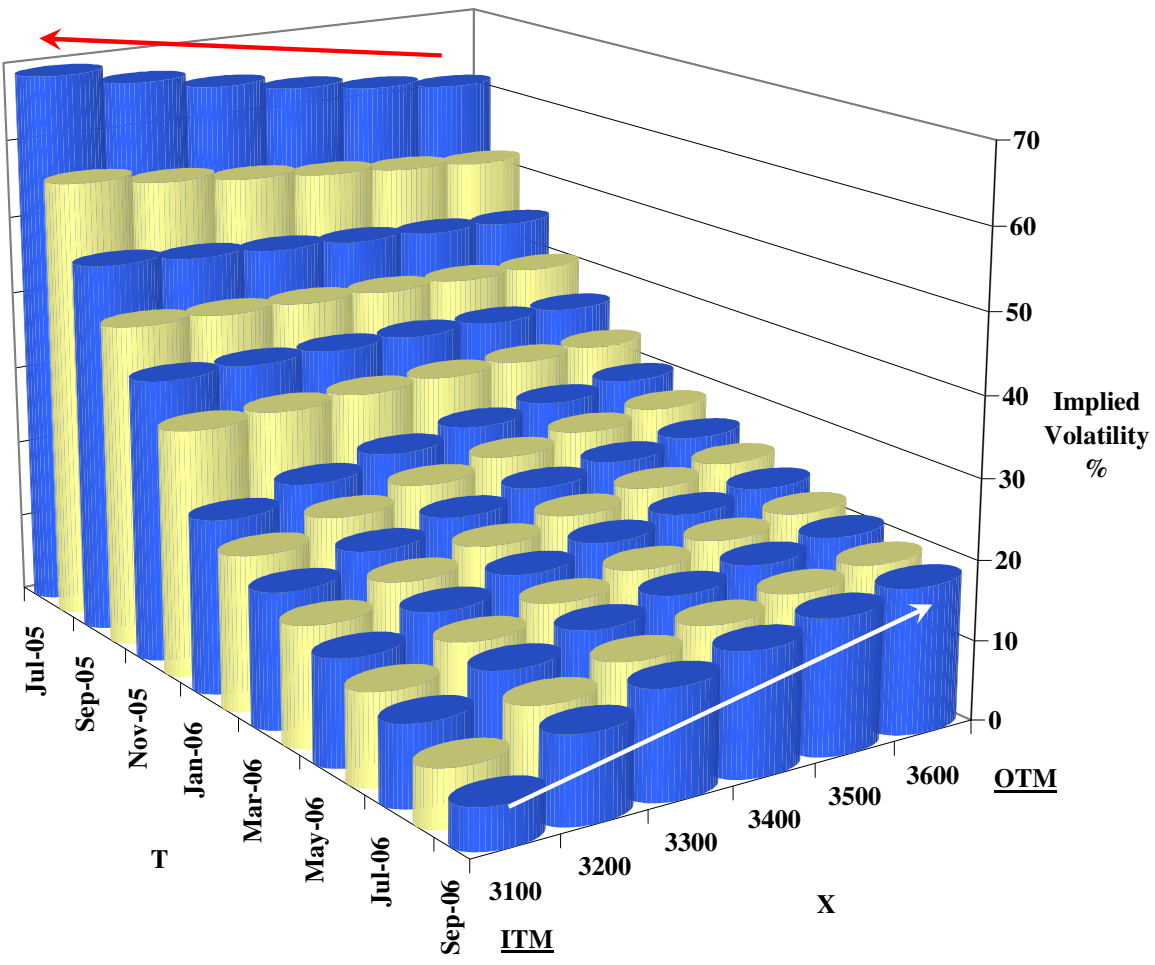

Figure (3.7): Implied Volatilities (Put, 21/02/05)

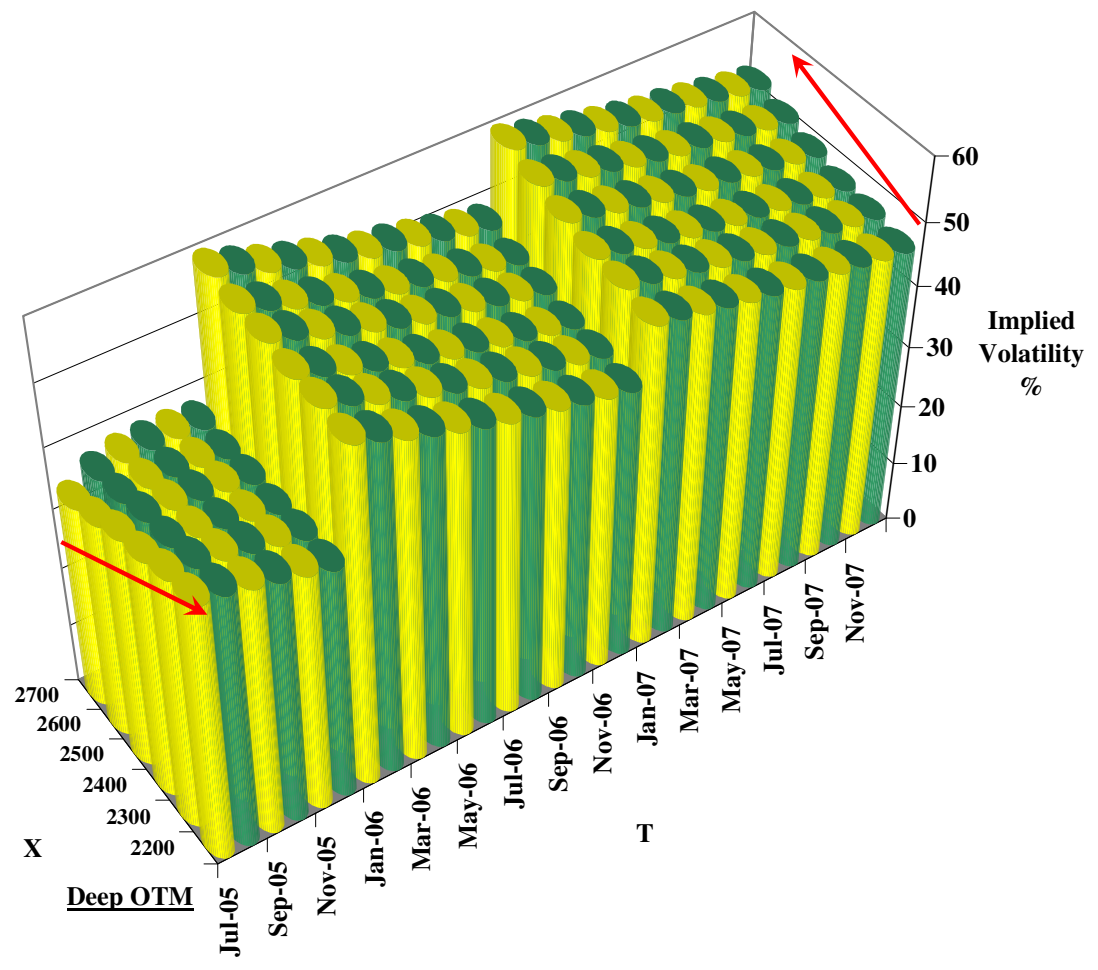


The volatilities could not be implied for put options based on the weekly data of following dates from 1-Aug-02 to 30-Aug-02 using the valuation technique of Asian options of the DerivaGem software package because all of the options' values were less than their intrinsic values. Therefore, the volatilities of all possible call options were implied, and only those of call options were valued on the above dates. On $21^{\text {st }}$ of February, the volatilities of all available call and put options were implied. Figures (3.6) and (3.7) show the implied volatility (I.V.) estimations for both call and put options on 21 February 2005.

In theory, there is a U-shape general form in the implied volatility curve which is shown in figure (3.8). The volatility becomes progressively high as an option position moves in either in-the-money (ITM) or out-of-the-money (OTM). However, the volatility is relatively low for at-the-money (ATM) options (Hull, 2002, p. 286).

\section{Figure (3.8): Volatility Smile}

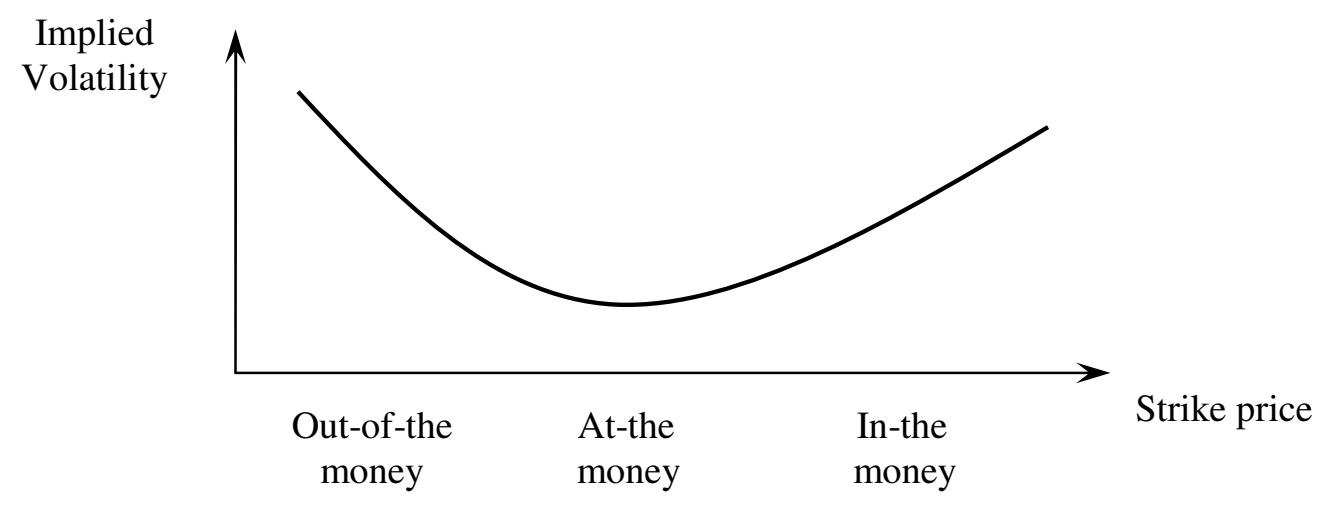

As can be seen from figures (3.1) through (3.5), implied volatility decreases as a call option approaches the deep out-of-the-money (OTM) position and increases as it becomes deep in-the-money (ITM). However, as the time to maturity increases, the 
implied volatility for a call option decreases as the option position becomes deep-in-themoney (ITM) and increases as the option approaches an out-of-the-money (OTM) position. This can be shown in figure (3.6).

For a put option, implied volatility increases as the option approaches the deep out-ofthe-money (OTM) position. However, volatility can also be high in the case where the option is deep in-the-money (ITM) because implied volatility can be high when an option is either deep in-the-money (ITM) or out-of-the-money (OTM), but low when the option is at-the-money (ATM).

As can be concluded from figures (3.6), (3.7) and table A1-6 in the appendix, for monthly settled call options July-05 through October- 05 , implied volatility is the highest when these options are deep in-the-money (ITM). However, for call options November05 through December-07, implied volatility is also the highest when these options approach a deep out-of-the-money (OTM) position. Implied volatility is lowest when the call options for February-07 until December-07 are at-the-money (ATM). In case of put options, implied volatility is the highest when all put options for July-05 through December-05 are deep out-of-the-money (OTM). For put options from January-06 to December-07, implied volatility is the highest when these options are deep in-the-money (ITM).

In addition, at the specific strike price, implied volatility decreases for either call or put options as the time to maturity increases. This is because an option will be more expensive if the remaining life of the option is longer. Finally, the general theory about implied volatility smile is proved by these implied volatility calculations. 
The appropriate implied volatility, which was used for valuing an option with a specific maturity date, was defined by the average of all implied volatilities with that specific maturity date. For instance, let us look at table A1-1 in appendix 1. As can be seen from this table, the calculation date was on 1 August 2002. There are three different implied volatilities obtained for each maturity date (month) because there are three different strike prices available, including $\$ 1,525, \$ 1,575$ and $\$ 1,600$. The appropriate implied volatility is the average of these calculated implied volatilities. As was mentioned before, the implied volatilities in a specific day (week) were used for valuing the options on the following day (week) of that specific day (week).

The historical volatility is calculated to be $21.51 \%$ and $14.94 \%$ per annum based on the monthly time series data on the LME copper prices since January 1973 until January 2005, and January 2003 until January 2005 respectively. The estimated historical volatility seems to be very low compared to the calculated implied volatilities. Additionally, the historical volatility may not fully incorporate the impact of information released to the market; therefore, using implied volatilities for options pricing is more appropriate than using historical volatilities in this analysis.

The following tables represent calculated theoretical option values using the Asian option valuation model of the Derivagem software on different dates with different strikes and maturities. 
Table 3.4: Valuations of Asian Options on 8-Aug-02 (Implied Vol. 01-Aug-02)

\begin{tabular}{|l|r|r|r|}
\hline \begin{tabular}{l|r|} 
Date of Data \\
CU Price (\$/t)
\end{tabular} & $\begin{array}{r}\text { 08-Aug-02 } \\
1467.00\end{array}$ & \\
\cline { 1 - 2 } T-Bill Discount Rates (\%) & \multicolumn{2}{|c|}{ Risk-Free Interest Rate } \\
\hline 3-month & 1.62 & 0.0164919 & $1.65 \%$ \\
6-month & 1.59 & 0.0162504 & $1.63 \%$ \\
1-year & 1.68 & 0.0173261 & $1.73 \%$ \\
\hline
\end{tabular}

\begin{tabular}{|c|c|c|c|c|c|c|c|c|}
\hline \multirow{3}{*}{$\begin{array}{l}\text { Strike } \\
\text { Price }\end{array}$} & \multicolumn{4}{|c|}{ 4Q-2002 CALL Options } & \multicolumn{4}{|c|}{ 2Q-2003 CALL Options } \\
\hline & \multirow{2}{*}{$\begin{array}{l}\text { Market } \\
\text { Values }\end{array}$} & \multicolumn{3}{|c|}{ Theoretical Values } & \multirow{2}{*}{$\begin{array}{l}\text { Market } \\
\text { Values }\end{array}$} & \multicolumn{3}{|c|}{ Theoretical Values } \\
\hline & & Oct-02 & Nov-02 & Dec-02 & & Apr-03 & May-03 & Jun-03 \\
\hline 1525 & 36 & 24.91 & 27.35 & 29.04 & 86 & 79.64 & 80.28 & 80.77 \\
\hline 1575 & 21 & 20.27 & 21.75 & 22.63 & 65 & 67.52 & 67.73 & 67.90 \\
\hline 1600 & 16 & 18.27 & 19.37 & 19.93 & 56 & 62.10 & 62.14 & 62.16 \\
\hline
\end{tabular}

Table 3.5: Valuations of Asian Options on 15-Aug-02 (Implied Vol. 08-Aug-02)

\begin{tabular}{|c|c|c|c|}
\hline $\begin{array}{l}\text { Date of Data } \\
\text { CU Price }(\$ / t)\end{array}$ & $\begin{array}{r}15 \text {-Aug-02 } \\
1474.00\end{array}$ & & \\
\hline \multicolumn{2}{|c|}{ T-Bill Discount Rates (\%) } & Risk-F & est Rate \\
\hline 3-month & 1.62 & 0.0164919 & $1.65^{c}$ \\
\hline & 1.63 & 0.0166626 & $1.67 c$ \\
\hline 1 -year & 1.78 & 0.0183763 & $1.84 \%$ \\
\hline
\end{tabular}

\begin{tabular}{|c|c|c|c|c|c|c|c|c|}
\hline \multirow{3}{*}{$\begin{array}{c}\text { Strike } \\
\text { Price }\end{array}$} & \multicolumn{4}{|c|}{ 4Q-2002 CALL Options } & \multicolumn{4}{|c|}{ 2Q-2003 CALL Options } \\
\hline & \multirow{2}{*}{$\begin{array}{l}\text { Market } \\
\text { Values }\end{array}$} & \multicolumn{3}{|c|}{ Theoretical Values } & \multirow{2}{*}{$\begin{array}{l}\text { Market } \\
\text { Values }\end{array}$} & \multicolumn{3}{|c|}{ Theoretical Values } \\
\hline & & Oct-02 & Nov-02 & Dec-02 & & Apr-03 & May-03 & Jun-03 \\
\hline 1525 & 36 & 21.37 & 24.20 & 26.21 & 87 & 77.74 & 78.72 & 79.40 \\
\hline 1575 & 20 & 17.26 & 19.07 & 20.20 & 66 & 65.64 & 66.15 & 66.47 \\
\hline 1600 & 15 & 15.50 & 16.90 & 17.69 & 57 & 60.24 & 60.56 & 60.72 \\
\hline
\end{tabular}

Table 3.6: Valuations of Asian Options on 22-Aug-02 (Implied Vol. 15-Aug-02)

\begin{tabular}{|c|c|c|c|}
\hline $\begin{array}{l}\text { Date of Data } \\
\text { CU Price }(\$ / t)\end{array}$ & $\begin{array}{r}22-\text { Aug-02 } \\
1486.50\end{array}$ & & \\
\hline \multicolumn{2}{|c|}{ T-Bill Discount Rates (\%) } & \multicolumn{2}{|c|}{ Risk-Free Interest Rate } \\
\hline $3-m$ & 1.65 & 0.0167986 & $1.68^{\circ}$ \\
\hline & 1.68 & 0.017178 & 1.72 \\
\hline 1-year & 1.85 & 0.0191128 & $1.91 \%$ \\
\hline
\end{tabular}

\begin{tabular}{|c|c|c|c|c|c|c|c|c|}
\hline \multirow{3}{*}{$\begin{array}{l}\text { Strike } \\
\text { Price }\end{array}$} & \multicolumn{4}{|c|}{ 4Q-2002 CALL Options } & \multicolumn{4}{|c|}{ 2Q-2003 CALL Options } \\
\hline & \multirow{2}{*}{$\begin{array}{l}\text { Market } \\
\text { Values }\end{array}$} & \multicolumn{3}{|c|}{ Theoretical Values } & \multirow{2}{*}{$\begin{array}{l}\text { Market } \\
\text { Values }\end{array}$} & \multicolumn{3}{|c|}{ Theoretical Values } \\
\hline & & Oct & Nov & Dec & & Apr & May & June \\
\hline 1525 & 37 & 20.35 & 23.64 & 25.96 & 90 & 80.52 & 81.58 & 82.33 \\
\hline 1575 & 21 & 16.48 & 18.64 & 19.99 & 68 & 68.10 & 68.65 & 69.00 \\
\hline 1600 & 15 & 14.82 & 16.53 & 17.50 & 59 & 62.55 & 62.88 & 63.08 \\
\hline
\end{tabular}


Table 3.7: Valuations of Asian Options on 30-Aug-02 (Implied Vol. 22-Aug-02)

\begin{tabular}{|l|r|r|r|}
\hline $\begin{array}{l}\text { Date of Data } \\
\text { CU Price (\$/t) }\end{array}$ & $\begin{array}{r}\text { 30-Aug-02 } \\
1500.00\end{array}$ & \\
\cline { 1 - 2 } T-Bill Discount Rates (\%) & \multicolumn{2}{|c|}{ Risk-Free Interest Rate } \\
\hline 3-month & 1.69 & 0.0172075 & $1.72 \%$ \\
6-month & 1.67 & 0.0170749 & $1.71 \%$ \\
1-year & 1.74 & 0.0179559 & $1.80 \%$ \\
\hline
\end{tabular}

\begin{tabular}{|c|c|c|c|c|c|c|c|c|}
\hline \multirow{3}{*}{$\begin{array}{l}\text { Strike } \\
\text { Price }\end{array}$} & \multicolumn{4}{|c|}{ 4Q-2002 CALL Options } & \multicolumn{4}{|c|}{ 2Q-2003 CALL Options } \\
\hline & \multirow{2}{*}{$\begin{array}{l}\text { Market } \\
\text { Values }\end{array}$} & \multicolumn{3}{|c|}{ Theoretical Values } & \multirow{2}{*}{$\begin{array}{l}\text { Market } \\
\text { Values }\end{array}$} & \multicolumn{3}{|c|}{ Theoretical Values } \\
\hline & & Oct & Nov & Dec & & Apr & May & June \\
\hline 1525 & 40 & 18.74 & 22.91 & 25.76 & 97 & 82.74 & 83.63 & 84.60 \\
\hline 1575 & 22 & 15.22 & 18.10 & 19.85 & 74 & 70.03 & 70.39 & 70.92 \\
\hline 1600 & 16 & 13.71 & 16.07 & 17.39 & 64 & 64.34 & 64.48 & 64.83 \\
\hline
\end{tabular}

Table 3.8: Valuations of Asian Options on 05-Sept-02 (Implied Vol. 30-Aug-02)

\begin{tabular}{|l|r|r|r|}
\hline $\begin{array}{l}\text { Date of Data } \\
\text { CU Price (\$/t) }\end{array}$ & $\begin{array}{r}\text { 05-Sep-02 } \\
1463.00\end{array}$ & \multicolumn{2}{|c|}{} \\
\hline \multicolumn{2}{|c|}{ T-Bill Discount Rates (\%) } & \multicolumn{2}{|c|}{ Risk-Free Interest Rate } \\
\hline 3-month & 1.61 & 0.0163897 & $1.64 \%$ \\
6-month & 1.58 & 0.0161473 & $1.61 \%$ \\
1-year & 1.66 & 0.0171163 & $1.71 \%$ \\
\hline
\end{tabular}

\begin{tabular}{|c|c|c|c|c|c|c|c|c|}
\hline \multirow{3}{*}{$\begin{array}{c}\text { Strike } \\
\text { Price }\end{array}$} & \multicolumn{4}{|c|}{ 4Q-2002 CALL Options } & \multicolumn{4}{c|}{ 2Q-2003 CALL Options } \\
\cline { 2 - 5 } \cline { 7 - 9 } & Market & \multicolumn{2}{|c|}{ Theoretical Values } & Market & \multicolumn{3}{c|}{ Theoretical Values } \\
\cline { 3 - 6 } & Values & Oct & Nov & Dec & Values & Apr & May & June \\
\hline $\mathbf{1 5 2 5}$ & $\mathbf{2 2}$ & 16.80 & 19.53 & 21.06 & $\mathbf{7 5}$ & 73.50 & 73.98 & 74.31 \\
$\mathbf{1 5 7 5}$ & $\mathbf{1 1}$ & 13.80 & 15.50 & 16.21 & $\mathbf{5 5}$ & 62.14 & 62.17 & 62.13 \\
$\mathbf{1 6 0 0}$ & $\mathbf{7}$ & 12.51 & 13.79 & 14.19 & $\mathbf{4 7}$ & 57.07 & 56.92 & 56.73 \\
\hline
\end{tabular}

Table 3.9: Valuations of Asian Options on 22-Feb-05 (Implied Vol. 21-Feb-05)

\begin{tabular}{|l|r|r|r|}
\hline $\begin{array}{l}\text { Date of Data } \\
\text { CU Price (\$/t) }\end{array}$ & $\begin{array}{r}\text { 22-Feb-05 } \\
\mathbf{3 3 6 7 . 0 0}\end{array}$ & \\
\hline \multicolumn{2}{|c|}{ T-Bill Discount Rates (\%) } & \multicolumn{2}{c|}{ Risk-Free Interest Rate } \\
\hline 6-month & 2.94 & 0.0300296 & $3.00 \%$ \\
1-year & 3.12 & 0.0321373 & $3.21 \%$ \\
2-year & 3.46 & 0.0363534 & $3.64 \%$ \\
3-year & 3.62 & 0.0388529 & $3.89 \%$ \\
\hline
\end{tabular}




\begin{tabular}{|c|c|c|c|c|c|c|c|}
\hline & \multicolumn{7}{|c|}{ 2005 CALL Options } \\
\cline { 2 - 8 } Strike & Market & \multicolumn{7}{|c|}{ Theoretical Values } \\
\cline { 3 - 8 } Price & Values & Jul. & Aug. & Sep. & Oct. & Nov. & Dec. \\
\hline 3100 & 208.17 & 188.06 & 195.54 & 202.35 & 207.88 & 213.26 & 218.55 \\
$\mathbf{3 2 0 0}$ & 168.40 & 158.12 & 162.48 & 166.41 & 169.56 & 172.57 & 175.53 \\
$\mathbf{3 3 0 0}$ & 134.84 & 132.35 & 134.19 & 135.81 & 137.06 & 138.18 & 139.26 \\
$\mathbf{3 4 0 0}$ & 106.92 & 110.30 & 110.20 & 110.04 & 109.84 & 109.52 & 109.20 \\
$\mathbf{3 5 0 0}$ & 83.99 & 91.56 & 90.01 & 88.55 & 87.31 & 85.97 & 84.66 \\
$\mathbf{3 6 0 0}$ & 65.42 & 75.72 & 73.15 & 70.80 & 68.86 & 66.86 & 64.93 \\
\hline
\end{tabular}

\begin{tabular}{|c|c|c|c|c|c|c|c|}
\hline \multirow{3}{*}{$\begin{array}{l}\text { Strike } \\
\text { Price }\end{array}$} & \multicolumn{7}{|c|}{2005 PUT Options } \\
\hline & \multirow{2}{*}{$\begin{array}{l}\text { Market } \\
\text { Values }\end{array}$} & \multicolumn{6}{|c|}{ Theoretical Values } \\
\hline & & Jul. & Aug. & Sep. & Oct. & Nov. & Dec. \\
\hline 2200 & 11.29 & 6.07 & 7.33 & 8.21 & 8.81 & 9.33 & 9.76 \\
\hline 2300 & 18.70 & 12.48 & 13.96 & 14.93 & 15.56 & 16.11 & 16.56 \\
\hline 2400 & 27.61 & 23.36 & 24.57 & 25.32 & 25.80 & 26.21 & 26.54 \\
\hline 2500 & 42.07 & 40.28 & 40.42 & 40.44 & 40.46 & 40.48 & 40.49 \\
\hline 2600 & 58.63 & 64.70 & 62.64 & 61.26 & 60.41 & 59.70 & 59.13 \\
\hline 2700 & 83.31 & 97.75 & 92.18 & 88.60 & 86.39 & 84.55 & 83.11 \\
\hline
\end{tabular}

\begin{tabular}{|c|c|c|c|c|c|c|c|c|c|c|c|c|c|}
\hline \multirow{3}{*}{$\begin{array}{c}\text { Strike } \\
\text { Price }\end{array}$} & \multicolumn{13}{|c|}{2006 CALL Options } \\
\hline & \multirow{2}{*}{$\begin{array}{l}\text { Market } \\
\text { Values }\end{array}$} & \multicolumn{12}{|c|}{ Theoretical Values } \\
\hline & & Jan. & Feb. & Mar. & Apr. & May & Jun. & Jul. & Aug. & Sep. & Oct. & Nov. & Dec. \\
\hline 3100 & 159.99 & 203.42 & 207.57 & 212.26 & 216.16 & 219.20 & 223.94 & 227.35 & 233.06 & 235.76 & 252.13 & 256.64 & 260.68 \\
\hline 3200 & 133.05 & 158.20 & 160.38 & 162.86 & 164.87 & 166.80 & 168.75 & 170.36 & 172.76 & 173.33 & 190.00 & 192.75 & 195.12 \\
\hline 3300 & 110.09 & 120.88 & 121.53 & 122.25 & 122.77 & 123.20 & 123.55 & 123.73 & 123.43 & 122.31 & 138.37 & 139.56 & 140.44 \\
\hline 3400 & 90.67 & 90.79 & 90.34 & 89.82 & 89.28 & 88.64 & 87.88 & 87.06 & 84.93 & 82.72 & 97.29 & 97.30 & 97.04 \\
\hline 3500 & 74.35 & 67.07 & 65.92 & 64.61 & 63.43 & 62.15 & 60.74 & 59.35 & 56.26 & 53.59 & 66.03 & 65.28 & 64.33 \\
\hline 3600 & 60.72 & 48.75 & 47.24 & 45.54 & 44.05 & 42.49 & 40.82 & 39.23 & 35.89 & 33.27 & 43.27 & 42.16 & 40.90 \\
\hline
\end{tabular}

\begin{tabular}{|c|c|c|c|c|c|c|c|c|c|c|c|c|c|}
\hline \multirow{3}{*}{$\begin{array}{l}\text { Strike } \\
\text { Price }\end{array}$} & \multicolumn{13}{|c|}{2006 PUT Options } \\
\hline & \multirow{2}{*}{$\begin{array}{l}\text { Market } \\
\text { Values }\end{array}$} & \multicolumn{12}{|c|}{ Theoretical Values } \\
\hline & & Jan. & Feb. & Mar. & Apr. & May & Jun. & Jul. & Aug. & Sep. & Oct. & Nov. & Dec. \\
\hline 2200 & 55.50 & 58.49 & 59.07 & 59.65 & 60.09 & 60.47 & 60.90 & 61.23 & 61.95 & 62.26 & 62.54 & 62.84 & 63.11 \\
\hline 2300 & 77.17 & 77.55 & 78.02 & 78.49 & 78.84 & 79.14 & 79.48 & 79.75 & 80.34 & 80.58 & 80.80 & 81.03 & 81.26 \\
\hline 2400 & 99.35 & 100.36 & 100.64 & 100.92 & 101.12 & 101.27 & 101.48 & 101.64 & 101.99 & 102.13 & 102.25 & 102.39 & 102.53 \\
\hline 2500 & 130.99 & 127.12 & 127.12 & 127.12 & 127.10 & 127.04 & 127.06 & 127.05 & 127.07 & 127.07 & 127.07 & 127.04 & 127.05 \\
\hline 2600 & 162.76 & 157.96 & 157.59 & 157.20 & 156.90 & 156.56 & 156.33 & 156.11 & 155.68 & 155.48 & 155.28 & 155.09 & 154.93 \\
\hline 2700 & 205.42 & 192.94 & 192.11 & 191.26 & 190.58 & 189.91 & 189.35 & 188.87 & 187.87 & 187.44 & 187.00 & 186.58 & 186.22 \\
\hline
\end{tabular}

\begin{tabular}{|c|c|c|c|c|c|c|c|c|c|c|c|c|c|}
\hline \multirow{3}{*}{$\begin{array}{l}\text { Strike } \\
\text { Price }\end{array}$} & \multicolumn{13}{|c|}{2007 CALL Options } \\
\hline & \multirow{2}{*}{$\begin{array}{l}\text { Market } \\
\text { Values }\end{array}$} & \multicolumn{12}{|c|}{ Theoretical Values } \\
\hline & & Jan. & Feb. & Mar. & Apr. & May & Jun. & Jul. & Aug. & Sep. & Oct. & Nov. & Dec. \\
\hline 3100 & 122.86 & 251.07 & 266.37 & 271.58 & 276.27 & 281.65 & 286.36 & 291.30 & 302.28 & 307.09 & 318.47 & 323.97 & 329.21 \\
\hline 3200 & 103.13 & 179.53 & 196.91 & 200.49 & 203.72 & 207.46 & 210.73 & 214.24 & 221.87 & 224.97 & 239.86 & 244.20 & 248.45 \\
\hline 3300 & 86.30 & 120.68 & 138.83 & 140.79 & 142.54 & 144.57 & 146.29 & 148.18 & 151.75 & 152.66 & 170.80 & 173.62 & 176.48 \\
\hline 3400 & 72.00 & 75.87 & 93.08 & 93.72 & 94.24 & 94.82 & 95.22 & 95.69 & 95.63 & 94.57 & 114.13 & 115.43 & 116.85 \\
\hline 3500 & 59.92 & 44.46 & 59.24 & 59.00 & 58.70 & 58.33 & 57.86 & 57.40 & 55.05 & 52.89 & 71.18 & 71.30 & 71.56 \\
\hline 3600 & 49.74 & 24.26 & 35.77 & 35.10 & 34.41 & 33.59 & 32.75 & 31.89 & 28.80 & 26.51 & 41.29 & 40.74 & 40.33 \\
\hline
\end{tabular}

\begin{tabular}{|c|c|c|c|c|c|c|c|c|c|c|c|c|c|}
\hline \multirow{3}{*}{$\begin{array}{l}\text { Strike } \\
\text { Price }\end{array}$} & \multicolumn{13}{|c|}{2007 PUT Options } \\
\hline & \multirow{2}{*}{$\begin{array}{l}\text { Market } \\
\text { Values }\end{array}$} & \multicolumn{12}{|c|}{ Theoretical Values } \\
\hline & & Jan. & Feb. & Mar. & Apr. & May & Jun. & Jul. & Aug. & Sep. & Oct. & Nov. & Dec. \\
\hline 2200 & 101.05 & 115.94 & 116.18 & 116.52 & 116.77 & 117.02 & 117.29 & 117.57 & 118.36 & 118.59 & 118.85 & 119.13 & 119.33 \\
\hline 2300 & 132.34 & 140.86 & 141.03 & 141.30 & 141.47 & 141.65 & 141.86 & 142.07 & 142.71 & 142.88 & 143.08 & 143.30 & 143.43 \\
\hline 2400 & 162.34 & 168.73 & 168.79 & 168.96 & 169.04 & 169.11 & 169.24 & 169.37 & 169.79 & 169.88 & 170.00 & 170.12 & 170.17 \\
\hline 2500 & 203.75 & 199.55 & 199.49 & 199.53 & 199.49 & 199.43 & 199.44 & 199.47 & 199.61 & 199.60 & 199.61 & 199.62 & 199.56 \\
\hline 2600 & 250.43 & 233.32 & 233.11 & 232.99 & 232.81 & 232.59 & 232.47 & 232.36 & 232.16 & 232.03 & 231.90 & 231.78 & 231.59 \\
\hline 2700 & 302.15 & 270.02 & 269.63 & 269.32 & 268.97 & 268.56 & 268.29 & 268.02 & 267.41 & 267.13 & 266.84 & 266.57 & 266.23 \\
\hline
\end{tabular}


A call option is more expensive when the option is likely to be in-the-money. In other words, a call option will be more expensive at a lower strike price, but less expensive at a higher strike price. In contrast, a put option is more expensive at a higher strike price, but less expensive at a lower strike price.

Before evaluating options' valuations, let us consider following definitions:

a) "Overpriced by theory" is the case that if an option's theoretically determined value is greater than its market observed value.

b) "Underestimated by theory" is the case that if an option's theoretical value is less than its market value.

Determined theoretical values do not exactly match the observed market prices of the options because there are some implied volatility biases. All these call options, which have been valued and illustrated by tables (3.4) through (3.8) are out-of-the-money.

As can be seen from these tables, theoretical call options values are overpriced when the call options are deep out-of-the-money (OTM). Table (3.9) represents theoretical valuations of monthly settled call and put options for 2005, 2006 and 2007. According to the results of this analysis, call options in 2005 are underestimated when they are in-themoney (ITM), but all out-of-the-money (OTM) call options for 2005 are overstated. However, all in-the-money (ITM) call options for 2006 and 2007 are overpriced and deep out-of-the-money (OTM) call options are undervalued. The put options for 2005, 2006 and 2007 are all out-of-the-money (OTM). The put options are overpriced as the option approaches a deep out-of-the-money (OTM) position. The table below summarizes the valuations of monthly settled Asian options since July-05 until December-07. 
Table 3.10: The Summary of Options' Valuations

\begin{tabular}{|c|c|c|c|}
\hline Year & $\begin{array}{c}\text { Option } \\
\text { Type }\end{array}$ & $\begin{array}{c}\text { Deep out-of-the-money } \\
\text { (OTM) }\end{array}$ & $\begin{array}{c}\text { Deep in-the-money } \\
\text { (ITM) }\end{array}$ \\
\hline \multirow{2}{*}{2005} & Call & Overpriced & Undervalued \\
& Put & Undervalued & - \\
\hline \multirow{2}{*}{2006} & Call & Undervalued & Overpriced \\
& Put & Overpriced & - \\
\hline \multirow{2}{*}{2007} & Call & Undervalued & Overpriced \\
& Put & Overpriced & - \\
\hline
\end{tabular}

In summary, put options are valued more accurately than call options because call options are more sensitive than puts. As more time remains until the expiration, theoretical call values are severely biased. 


\section{CHAPTER IV:}

\section{BACKGROUND OF ECONOMETRIC COMMODITY MODELS AND LITERATURE REVIEW}

Chapter V builds copper price prediction model, which is important to risk management strategies and their decisions. Before building any econometric model, it is important to review earlier developed and currently used models, if they are available. Therefore, six econometric models in non-ferrous metals' market will be briefly reviewed in this chapter.

1. Desai's (1966) model: The basic variables, which are used in econometric models of commodity markets are usually supply (or production), demand (or consumption) and price (Slade, 1984, p. 14). Desai's model was built for the world tin industry and a main focus of this model was on the demand side of the tin market. A simplified version of this model is as follows:

$$
\left\{\begin{array}{l}
D_{t}=f\left\{V E A_{t}\right\} \\
S_{t}=f\left\{S_{(t-1)}\right\} \\
P_{t}=f\left\{\frac{I_{(t-1)}}{D_{t}}\right\} \\
I_{t}=I_{(t-1)}+S_{t}-D_{t}
\end{array}\right.
$$

$D_{t}, S_{t}, P_{t}=$ the demand, supply and price at time $t$, respectively

$\mathrm{I}_{\mathrm{t}} \quad=$ the inventory level at time $\mathrm{t}$

$\mathrm{VEA}_{\mathrm{t}}=$ the value of some aggregate economic activity variable at time $\mathrm{t}$ 
As stated, this model assumes that neither demand nor supply is sensitive to price, according to Desai's analysis. The main importance of this model was the assumption that the value of some aggregate economic activity significantly affects the demand for a metal and this assumption is followed by latter models of the metals markets.

2. Fisher-Cootner-Baily (FCB) (1972) Model: This model was built for the world copper industry. Unlike Desai's model, the FCB model found both supply and demand to be sensitive to the price of copper. In addition, FCB have argued that demand is also related to the price of a substitute. Aluminum is a substitute base metal for copper. Following is a simplified version of the FCB model.

$$
\left\{\begin{array}{l}
D_{t}=f\left\{V E A_{t}, P_{(t-1)}, P_{(t-1)}^{S}, D_{(t-1)}\right\} \\
S_{t}=f\left\{P_{t}, S_{(t-1)}\right\} \\
P_{t}=f\left\{\frac{\Delta I_{(t-1)}}{\Delta D_{(t-1)}}, P_{(t-1)}^{W}-P_{(t-1)}, P_{(t-1)}\right\} \\
\Delta I_{t}=S_{t}-D_{t}
\end{array}\right.
$$

$$
\begin{aligned}
& \mathrm{P}_{\mathrm{t}}=\text { the U.S. price of copper at time } \mathrm{t} \\
& \mathrm{P}^{\mathrm{W}}{ }_{\mathrm{t}}=\text { the world price of copper at time } \mathrm{t} \\
& \mathrm{P}_{\mathrm{t}}^{\mathrm{S}}=\text { the price of a substitute (aluminum) at time } \mathrm{t}
\end{aligned}
$$

There are several main differences between the Desai's model and the FCB model. First, distributed lag variables have been added to all equations in the FCB model. Second, two different copper prices including the U.S. price and the world price are used in the FCB model. Third, the price of a substitute is included. 
3. Charles River Associates (CRA) Model: CRA have estimated econometric models for many important mineral industries and one of its models was built for the primary aluminum industry in 1971. The value of aggregate economic activity, the price of an underlying metal, and the price of a substitute are used as exogenous variables for explaining demand for the underlying metal, primary aluminum. The unique aspect of the CRA model is that it used the long-run average cost (LRAC) as an explanatory variable in the price equation. Demand is a downward sloping function of price and price is a function of LRAC and is independent of the quantity produced; therefore, supply is obtained as the intersection of the price and demand (Slade, 1984, p.19). The system equations below demonstrate a simplified version of the CRA model.

$$
\left\{\begin{array}{l}
D_{t}=f\left\{V E A_{t}, P_{(t-i)}, P_{(t-i)}^{S}\right\}, i=0, \ldots, 3 \\
P_{t}=f\left\{L R A C_{t}, P_{(t-1)}\right\}
\end{array}\right.
$$

LRAC $_{\mathrm{t}}=$ the long run average cost at time $\mathrm{t}$

In these models, copper and aluminum markets have been connected only by the price of the exogenous substitute.

\section{Copper-Aluminum Substitution (CAS) (1979) Model:}

This model was built by Margaret E. Slade in 1979 in line with her Doctor of Philosophy dissertation.

A simplified version of the model is as follows: 


$$
\left\{\begin{array}{l}
\sum_{J} D_{t}^{J}=f\left\{V E A_{t}, P_{t}^{C, A}, \sum_{J} D_{(t-1)}^{J}\right\} ; J=C, A \\
\frac{D_{t}^{C}}{D_{t}^{A}}=f\left\{P_{(t-i)}^{A}, P_{(t-i)}^{C}\right\} ; i=0, \ldots, 3 \\
S_{t}^{J}=f\left\{P_{t}^{J}, V E A_{t}, \frac{I_{t}^{J}}{D_{t}^{J}}, S_{(t-1)}^{J}\right\} ; J=C, A \\
P_{t}^{J}=f\left\{P_{i, t}^{J}, R_{t}^{J}, T_{t}^{J}, \frac{I_{t}^{J}}{D_{t}^{J}}, P_{(t-1)}^{J}\right\} ; J=C, A
\end{array}\right.
$$

$\mathrm{D}_{\mathrm{t}}^{\mathrm{J}}=$ demand for metal $\mathrm{J}$ at time $\mathrm{t}$

$\mathrm{VEA}_{\mathrm{t}}=$ the value of some aggregate economic activity variable at time $\mathrm{t}$

$\mathrm{P}_{\mathrm{t}}^{\mathrm{C}, \mathrm{A}}=$ weighted average of copper and aluminum prices at time $\mathrm{t}$

$\mathrm{P}_{\mathrm{t}}^{\mathrm{J}}=$ the price of metal $\mathrm{J}$ at time $\mathrm{t}$

$\mathrm{S}_{\mathrm{t}}^{\mathrm{J}}=$ primary supply of metal $\mathrm{J}$ at time $\mathrm{t}$

$\mathrm{I}_{\mathrm{t}}^{\mathrm{J}}=$ the level of inventories of metal $\mathrm{J}$ at time $\mathrm{t}$

$\mathrm{P}_{\mathrm{i}, \mathrm{t}}^{\mathrm{J}}=$ the price of the $\mathrm{i}^{\text {th }}$ input to primary production of metal $\mathrm{J}$ at time $\mathrm{t}$

$\mathrm{R}_{\mathrm{t}}^{\mathrm{J}}=$ a measure of the quality of resource $\mathrm{J}$ at time $\mathrm{t}$

$\mathrm{T}_{\mathrm{t}}^{\mathrm{J}}=$ a measure of the state of technology in the primary production of metal $\mathbf{J}$ at time $\mathrm{t}$

In this model, the average yield of ores mined was used as the best measure of ore quality $\left(\mathrm{R}_{\mathrm{t}}^{\mathrm{J}}\right)$. In addition, technological improvement $\left(\mathrm{T}_{\mathrm{t}}^{\mathrm{J}}\right)$ was measured by higher labor productivity.

The level of inventories is a determinant of production as well as of price. In addition, a producer can anticipate the demand for a product and accordingly produce the product, so the aggregate economic activity variable is also used a determinant of production in the CAS model. The uniqueness of the CAS model is that a measure of the state of 
technology in the primary production of a metal is used as an independent variable of a metal price equation (Sources of these models' reviews: Slade, 1984, p.14-23).

5. Istvan Dobozi (ID) Model: This model is a single-equation model that tries to explain world metal consumption. Total metal consumption is a function of the level of overall economic activity, the lagged price of the underlying metal and the lagged price of oil (Tilton, 1990, p. 117).

$$
\begin{aligned}
& \qquad Y_{t}=A \cdot Q_{t}^{B} \cdot P_{(t-1)}^{C} \cdot P_{O I L(t-1)}^{D} \cdot e^{u} \\
& \mathrm{Y}_{\mathrm{t}}=\text { total metal consumption in year } \mathrm{t} \\
& \mathrm{Q}_{\mathrm{t}}=\text { total GDP in year } \mathrm{t} \\
& \mathrm{P}_{(\mathrm{t}-1)}=\text { metal's world market price, lagged one year } \\
& \mathrm{P}_{\mathrm{OIL}(\mathrm{t}-1)}=\text { price of oil, lagged one year } \\
& \mathrm{A}=\text { intercept } \\
& \mathrm{C}=\text { price elasticity of metal demand } \\
& \mathrm{D}=\text { elasticity of metal demand with respect to world price of oil } \\
& \mathrm{U}=\text { error term in year } \mathrm{t}
\end{aligned}
$$

The price of oil is being used in this model because energy costs are a major part of the costs of copper production. The price of oil is a useful proxy variable for the cost of energy in producing copper. This model tried to explain total metal consumption in year $t$, not total metal supply in year $t$, so the price of oil is lagged by one year. The price of oil in the previous period affects the metal supply in that previous period (t-1), but affects the 
metal consumption in the next period $(\mathrm{t})$ because of the consumers' reaction against the producers' behavior. Thus, the price of oil is lagged by one period in the ID model.

6. Bloomsbury Mineral Economics (BME) Model: Financial institutions, consulting groups and research service organizations provide various metal price models. For instance, Bloomsbury Minerals Economics (BME) Limited, which researches metals markets, has an interactive LME metal price model for six base metals including aluminum, copper, nickel, zinc, lead, tin, and precious metals including gold and silver. According to the BME model, the LME copper price is a function of LME stock level (ST), global industrial production (IP) growth (year-on-year IP growth), and an index of the strength of the U.S. dollar against other major currencies (USDI).

$$
P_{t}=f\left\{S T_{t}, I P_{t}, U S D I_{t}\right\}
$$

Single equation methods can devote greater attention to econometric problems of autocorrelation. On the other hand, it is necessary to use a simultaneous system instead of a single econometric model when demand, supply or prices of a commodity are explained because a single econometric model lacks the theoretical basis of a simultaneous system. Consequently, simultaneous econometric models should be specified and built when copper supply, demand and market prices are determined. 


\section{CHAPTER V:}

\section{MODEL DEVELOPMENT FOR}

\section{PRICE PREDICTION}

Price risk management and hedging programs for commodities are closely linked to cycles in and trends of commodity prices. Someone who hedges throughout the copper price cycle will come out overall the same as someone who does not hedge at all over the whole cycle in copper prices and price fluctuations, assuming no broker's fees. In other words, a producer hedging when prices are increasing does less well, but has a smoother return. In contrast, a producer who does not hedge does less well when prices are falling, and has a more volatile return. In the long run, the overall returns may be similar between hedgers and non-hedgers. Therefore, the only way to profit from hedging is to use price prediction tools to estimate in advance which periods will be the increasing phase of the price cycle and not hedge in them. For this reason, accurate price prediction is important and would be essential to manage market price risk successfully.

Although perfect forecasting is impossible, accurate forecasting is possible and useful. Thus, the purpose of this chapter is to model copper prices using a simultaneous equation system (two-stage least squares regression) and important key variables for explaining prices. 


\subsection{SPECIFICATION OF THE MODEL}

The most important theories and models in economics and business usually suggest that the important endogenous variables are determined simultaneously. The price of a commodity is simultaneously determined by the interaction of producers and consumers in a market; thus, demand, supply and prices are obviously simultaneous (Pindyck, Rubinfeld, 1981, p. 180). All of the endogenous variables must necessarily be correlated with the error terms in all of the equations; hence, an important assumption, which states that in order for Ordinary Least Squares (OLS) estimators to be the best possible, all explanatory variables should be uncorrelated with the error term, is violated in a simultaneous model (Studenmund, 2001, p. 462). A supply and demand simultaneous model with three-equation has the following general form.

$$
\left\{\begin{array}{l}
Q_{t}^{D}=\alpha_{0}+\alpha_{1} \cdot P_{t-1}+\alpha_{2} \cdot \chi_{t}^{D 1}+\ldots+\alpha_{n} \cdot \chi_{t}^{D n}+u_{t}^{D} \\
Q_{t}^{S}=\beta_{0}+\beta_{1} \cdot P_{t-1}+\beta_{2} \cdot \chi_{t}^{S 1}+\ldots+\beta_{k} \cdot \chi_{t}^{S k}+u_{t}^{S} \\
Q_{t}^{D}=Q_{t}^{S}
\end{array}\right.
$$

where, $\alpha_{0}$ and $\beta_{0}$ are the intercept terms, $\mathrm{X}^{\mathrm{Di}}{ }_{\mathrm{t}}$ and $\mathrm{X}^{\mathrm{Si}}{ }_{\mathrm{t}}$ are row vectors of observations on exogenous or predetermined variables that appear respectively in the demand and supply functions, $\alpha_{1}$ to $\alpha_{\mathrm{n}}$ and $\beta_{1}$ to $\beta_{\mathrm{n}}$ are corresponding parameters, and $\mathrm{u}_{\mathrm{t}}^{\mathrm{D}}$ and $\mathrm{u}_{\mathrm{t}}^{\mathrm{S}}$ are the error terms in the demand and supply functions. Economic theory predicts $\alpha_{1}<0$ and $\beta_{1}>0$. Lagged price of the underlying commodity is an essential variable for explaining demand and supply of the underlying commodity. In a specific year t, producers and consumers do not know the prevailing price of the underlying commodity. Their decisions in year $t$ 
regarding production and consumption will be based on last year's ( $\mathrm{t}-1)$ price because they only know that price. Therefore, this variable is included both structural equations of demand and supply in the simultaneous system (5.1). The Cobweb model explains more detail about choosing the lagged price as an important exogenous variable.

The model (5.1) is explaining how the market variables including supply, demand and price interact and how they are affected by the exogenous variables. The supply equation, demand equation and equilibrium condition determine the market price and the quantity supplied (and demanded) when the competitive market is in equilibrium. Therefore, the simultaneously (jointly) determined variables $\mathrm{Q}^{\mathrm{D}}, \mathrm{Q}^{\mathrm{S}}$ and $\mathrm{P}_{\mathrm{t}-1}$ are called endogenous variables and their behaviors are explained by the model. On the other hand, the system equation contains $(n+k)$ variables, whose values are not determined directly within the system. These variables are called exogenous variables and their behaviors are completely explained outside the model system. The predetermined variables include all exogenous and lagged endogenous variables. Viewed as a system of equations, the model (5.1) has three endogenous variables and $(n+k+1)$ predetermined variables. These socalled predetermined variables help to cause the movement of the endogenous variables within the system (Pindyck, Rubinfeld, 1981, p. 181).

\section{A) Specification of the Variables:}

As mentioned before, an important concern of a model of a commodity market is the simultaneous estimation demand, supply and prices. The Cobweb model is useful for explaining the volatility in commodity prices because it was originally developed for agricultural commodities. 
The Cobweb model is given by the system equation (5.2) for the copper market:

$$
\left\{\begin{array}{l}
D_{t}=\alpha_{0}-\alpha_{1} \cdot P_{t-1} \\
S_{t}=\beta_{0}+\beta_{1} \cdot P_{t}^{*}+\varepsilon_{t} \\
D_{t}=S_{t}
\end{array} \quad \alpha_{1}>0 \text { and } \beta_{1}>0\right.
$$

$\mathrm{D}_{\mathrm{t}}=$ demand for copper in period $\mathrm{t}(\mathrm{t}$ - refers to time)

$\mathrm{S}_{\mathrm{t}}=$ supply of copper in period $\mathrm{t}$

$\mathrm{P}_{\mathrm{t}-1}=$ market price of copper in period $(\mathrm{t}-1)$

$\mathrm{P}_{\mathrm{t}}^{*}=$ price that producers expect to prevail at period $\mathrm{t}$

$\varepsilon_{\mathrm{t}}=\mathrm{a}$ zero mean stochastic supply shock and parameters $\alpha_{0}, \beta_{0}, \alpha_{1}$ and $\beta_{1}$ are all positive such that $\alpha_{0}>\beta_{0}$.

There are no exogenous explanatory variables in the Cobweb model (5.2).

At the market-clearing price of $\left\{\mathrm{P}_{\mathrm{t}}\right\}$, consumers buy as much copper as they desire. At the time of production, producers do not know the prevailing price; therefore, their supply decision will be based on the expected price of copper $\left\{\mathrm{P}_{\mathrm{t}}^{*}\right\}$. Assuming that last year's price is the expected price at time $t$, then $\left\{\mathrm{P}_{\mathrm{t}}^{*}\right\}=\mathrm{P}_{(\mathrm{t}-1)}$. A competitive market requires that the quantity supplied equals the quantity demanded, once the copper is produced. The actual quantity produced is then $S_{t}=\left\{\beta_{0}+\beta_{1} \cdot P_{t}^{*}+\varepsilon_{t}\right\}$.

$$
\text { Hence, } \quad\left\{\beta_{0}+\beta_{1} \cdot P_{t}^{*}+\varepsilon_{t}\right\}=\left\{\alpha_{0}-\alpha_{1} \cdot P_{t-1}\right\}
$$

If the stochastic error term is zero $\left(\varepsilon_{\mathrm{t}}=0\right)$ and $\left\{\mathrm{P}_{\mathrm{t}}^{*}\right\}=\mathrm{P}_{(\mathrm{t}-1)}$, then following equations are developed from equations (5.2) and (5.3). 


$$
\begin{gathered}
P_{t-1}=\frac{\alpha_{0}-\beta_{0}}{\beta_{1}+\alpha_{1}} \\
S_{(D=S)}=\beta_{0}+\beta_{1} \cdot \frac{\alpha_{0}-\beta_{0}}{\beta_{1}+\alpha_{1}}=\frac{\alpha_{0} \cdot \beta_{1}+\beta_{0} \cdot \alpha_{1}}{\beta_{1}+\alpha_{1}}
\end{gathered}
$$

These are all constant because there are no exogenous explanatory variables in the model (5.2). However, if exogenous explanatory variables are included in the model, simultaneous endogenous variables will not be constant.

\section{Figure (5.1): The Cobweb Model}

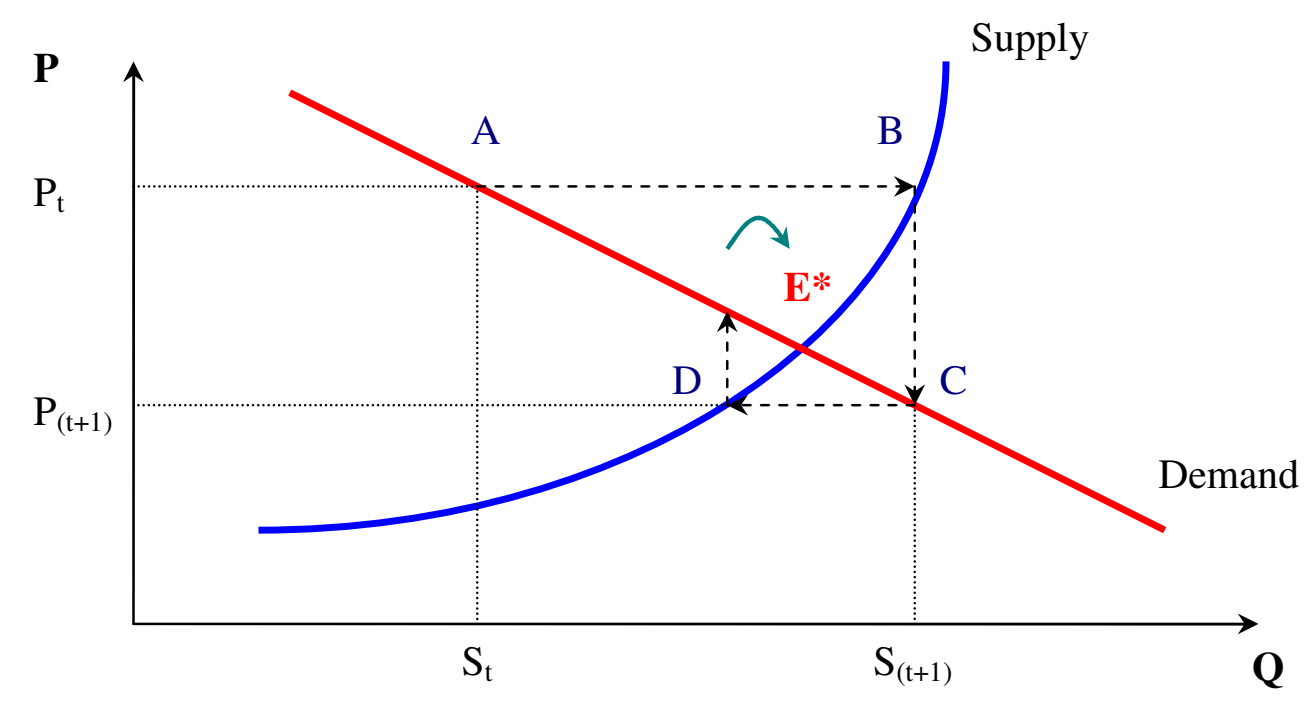

Assume there are no inventories. Suppose that the equilibrium quantity $\mathrm{S}^{*}$ is planned to be produced in time t. Nevertheless, let there be a negative supply shock, so the actual quantity produced turns out to be $S_{t}$, where $S_{t}<S^{*}$. For the quantity $S_{t}$, consumers are willing to pay $\mathrm{P}_{\mathrm{t}}$; thus, market clearing in time $t$ occurs at point $\mathrm{A}$. Assume all subsequent values of the supply shock are zero. This means $\varepsilon_{(t+1)}=\varepsilon_{(t+2)}=\ldots=0$. Producers expect that the price at producing time $(\mathrm{t}+1)$ to be the price of the previous period $\mathrm{t}$; therefore, 
$\mathrm{P}_{(t+1)}^{*}=\mathrm{P}_{\mathrm{t}}$. In this case, producers produce the market quantity $\mathrm{S}_{(\mathrm{t}+1)}$ at point $\mathrm{B}$; however, consumers are willing to pay only $\mathrm{P}_{(t+1)}$ for the quantity $\mathrm{S}_{(t+1)}$. Consumers will buy quantity $\mathrm{S}_{(\mathrm{t}+1)}$ only if the copper price falls to $\mathrm{P}_{(\mathrm{t}+1)}$ at point $\mathrm{C}$. Then market equilibrium shifts to point $\mathrm{D}$ due to the producers' reaction against the consumers' behavior. The process continually repeats until the market equilibrium is obtained at point $\mathrm{E}^{*}$ (Enders, 2004, p. 17-19). Therefore, supply, demand and market prices are obviously determined simultaneous, so they should be determined jointly.

Without speculation, commodity prices would be determined by ordinary supply and demand theory. Nevertheless, there is always speculation in a commodity market; thus, other explanatory variables should be specified and included in the simultaneous model. Let us start with the structural equation of demand.

Stock adjustments should be considered when reporting copper demand because inventory changes cause a divergence between reported consumption and actual demand trends. In other words, a metal balance requires that the difference between domestic consumption and production of the metal be equal to net imports minus changes in inventories (Tilton, 1990. p.23; Slade, 1984, p. 252). Thus, the change in total inventories of refined copper should be included in the estimation of refined copper demand.

$$
\begin{aligned}
& \left\{\begin{array}{l}
C_{t}-P_{t}=I m_{t}-E x_{t}-\Delta \operatorname{In} v_{t} \\
S_{t}=P_{t}+I_{t} \\
D_{t}=C_{t}+\Delta \operatorname{In} v_{t}+E x_{t}
\end{array}\right. \\
& \mathrm{C}_{\mathrm{t}}=\text { consumption } \quad \mathrm{P}_{\mathrm{t}}=\text { production } \quad \operatorname{Im}_{\mathrm{t}}=\text { imports } \\
& \text { Ex }_{\mathrm{t}}=\text { exports } \quad \mathrm{S}_{\mathrm{t}}=\text { supply } \quad \mathrm{D}_{\mathrm{t}}=\text { demand } \\
& \Delta \operatorname{Inv}_{\mathrm{t}}=\text { changes in total inventories }=\text { Additions }- \text { Withdrawals }
\end{aligned}
$$


Both imports and exports are omitted from the system because the world copper market is being examined. The demand for copper is defined as total consumption plus changes in total inventories.

According to the Cobweb model, the demand is related to the lagged value of price of the underlying commodity-copper because the demand for copper responds with delay when copper prices move. In the beginning of year t, consumers and producers do not know at what point copper price will be settled; thus, their purchasing and producing decisions will be based on a price of the previous year (t-1), so prices are lagged by one year in both demand and supply equations. The expected sign of the coefficient estimate of the lagged price variable is negative in the demand equation, according to the law of demand. In addition, metal demand (consumption) depends on external influences such as the level and trend of overall economic development and activity. The world GDP is a useful variable to measure aggregate economic activity and the overall level of income in the world. Additionally, business cycles are the periodic fluctuations in GDP around its long-term growth trend, so GDP includes some aspects of the business cycles. The demand for copper increases as global economic activity intensifies, business cycle expanses and income increases. Hence, the expected sign of the coefficient estimate of the GDP variable is positive. The price of close substitutes also determines the demand for a particular commodity. Therefore, the world demand for copper is typically assumed to be a function of the price of copper, the price of close substitute materials and the aggregate economic activity variable (Tilton, 1990. p. 30). Although the price of a substitute (aluminum in this case) is a useful variable for explaining the demand for copper, it is excluded from the demand function because the primary focus of building a 
simultaneous system in this chapter is forecasting the price of copper. Forecasting aluminum price is another big research topic; therefore, if aluminum price is included in the system, this would significantly reduce the accuracy of copper price forecasts because copper and aluminum are close substitutes. In addition, if the lagged price of aluminum is included as an exogenous explanatory variable in the demand equation, it causes problems for the results of the two-stage least squares regression of the supply equation as shown in table A6-2 of appendix 6 (see appendix 6). In fact, t-statistics reduced for the lagged price of copper, the price of oil and the 6-month lagged LIBOR (1Y) variables in the supply equation if the lagged price of aluminum is included in the demand equation. As can be seen from table A6-2 in appendix 6, two essential variables including the lagged price of copper and the price of oil in the supply equation had insignificant tscores. In addition, the price of oil variable had incorrect sign. All these outcomes were caused by if the lagged price of aluminum is included in the system; therefore, empirical analysis result concludes that the variable should be excluded from the demand equation.

A variable which is a proxy for the industrial cycle can be useful in the structural equation of demand. Copper is an industrial base metal; so it is essential to consider a variable of the global industrial production (IP) cycle or year-over-year IP growth. According to the LME (the official web page: www.lme.co.uk), $48 \%$ of world copper is used for building construction, $17 \%$ in electrical applications, $16 \%$ in general engineering, $8 \%$ in light engineering, $7 \%$ in transportation, and $4 \%$ in other industries. The general and light engineering markets include military and aerospace, power generation, vehicle applications, marine applications, appliances, factory automation, pumps and compressors and consumer electronics. 
The use of IP as an explanatory variable to explain copper price in a demand equation became popular in the 1990s and emphasized the importance of the IP cycle in explaining metal prices. Therefore, many research organizations, including BME, used both IP and traditional variables in their price models. Briefly, the IP growth variable is routinely used in price models and is measured in percentage terms, not by dollar values like total industrial production in the U.S. dollars. Therefore, this variable does not have the same meaning as the GDP variable. The demand for copper rises as global IP growth increases; therefore, the expected sign of the coefficient estimate of IP cycle is positive. Consequently, the demand function must be declining with respect to the price of copper and ascending with respect to the GDP and IP.

Table 5.1: Correlation Coefficients between Demand and its Exogenous Variables

\begin{tabular}{|c|c|c|c|}
\hline & $\mathbf{P}_{(\mathbf{t}-\mathbf{1})}$ & $\mathbf{G D P}_{\mathbf{t}}$ & $\mathbf{I P \%}_{\mathbf{t}}$ \\
\hline $\mathbf{D}_{\mathbf{t}}$ & -0.1312 & 0.7749 & 0.2516 \\
\hline
\end{tabular}

The table above represents simple correlation coefficients between the endogenous demand variable and its exogenous variables. Consequently, the structural equation of demand is specified with expected signs as follows:

$$
\left\{\begin{array}{l}
D_{t}=f\left\{P_{(t-1)}^{(-)}, G D P_{t}, I P(\stackrel{(+)}{\%})_{t}\right\} \\
D_{t}=C_{t}+\Delta I n v_{t}
\end{array}\right.
$$

According to the Cobweb model, the supply is also related to the lagged value of price of the underlying commodity-copper. The quantity supplied increases as price increases, so the expected sign of the coefficient estimate is positive for the lagged price 
variable in the structural equation of supply. The second exogenous variable that explains copper supply is the variable measuring technological change. Technological change reduces the quantity of inputs required to produce a unit of output. Because time-series data on the input-output ratios are not available, it is appropriate to use time (T) as a proxy for changes in this ratio. Technological advance is a main factor for increasing the supply of a commodity and it leads to reductions in the cost of supply. In the copper industry, for instance, low cost solvent extraction-electro winning (SX-EW) technology has substantially increased refined copper production during 1990s. Technology improvements push supply up, so copper price falls with improvements in technology (as measured by time). Therefore, expected sign of the coefficient estimate of the time variable is positive in the structural equation of supply.

It is essential to consider the cost of production when explaining the supply of a certain commodity. Energy is a major element in the cost of copper production. Approximately 34\% of total production cost is for energy in world copper refineries.

The processes of copper smelters and refineries are very energy-intensive. Major energyconsuming equipment in copper smelters and refineries include process-heating equipments such as dryers, matte grinding and drying mills, smelting and refining furnaces, steam generators (boilers), pumps, air compressors and electric motors, the sulfuric acid plant, and extensive emission control facilities (DOE, 2004).

As a result, the price of oil is a useful proxy variable for the cost of energy in producing copper. High energy prices increase the cost of copper production, so it has a negative effect on copper production. Therefore, the expected sign of the oil price coefficient estimate is negative in the structural equation of supply. 
The next exogenous variable that explains copper supply is the U.S. dollar index. According to the ICSG statistics, the U.S. produces approximately $8.3 \%$ of total refined copper production of the world. Approximately $14.67 \%$ of total refined copper usage of the world is from U.S. consumption. Copper prices are quoted in U.S. dollars, so the strength and weakness of the dollar index has less effect on the U.S. copper producers (or exporters) and consumers (or importers). However, changes in the value of the U.S. dollar significantly influence foreign producers and consumers because domestic currency devaluation has a positive influence on the gross revenue of the domestic exporters, but negative effects to the domestic buyers or importers. Let us consider a brief example:

Chile produced $37.26 \%$ of total world copper mine production in 2004 . This copper is exported to the global copper market. The currency exchange rate of the Chilean peso (CLP) was 586.79 against one U.S. dollar (i.e. 1 USD $=586.79$ CLP) as of 16:00 EST Friday, June 3, 2005. Assume, a Chilean producer intends to export 1000 tonnes of copper at current market price of $\mathrm{P}(\$ / \mathrm{t})$. According to the current exchange rate, the producer's sales revenue is $[1000 \mathrm{t} * \mathrm{P}(\$ / \mathrm{t}) * 586.79 \mathrm{CLP} / \$]$ in pesos. If the exchange rate of CLP weakens against the USD, for instance, to 590.80 (i.e. 1 USD $=590.80$ CLP), a Chilean producer (exporter) would make an additional profit of $[1000 \mathrm{t} * \mathrm{P}(\$ / \mathrm{t}) * 4.01$ $\mathrm{CLP} / \$$ ] in pesos (where: $4.01=590.80-586.79$ ) due to the devaluation of the domestic currency (CLP) against the U.S. dollar.

Consequently, increases in the U.S. dollars value stimulates foreign producers and exporters, but may not influence the U.S. producers. There is another reason to include the value of the U.S. dollar in the simultaneous system. The value of the currency has a 
strong relationship to metal price. The goal of building the simultaneous model is to get the price equation from the system, so the relative strength and weakness of the U.S. dollar is important. The U.S. dollar index is used as a measure of the relative value of the dollar; hence, it is selected as an input variable for the supply equation of the simultaneous model. A high dollar index means that the dollar is strong and less dollars are required to purchase a unit of metal. By contrast, a weak dollar index means more dollars required to purchase the same unit of metal. Therefore, there is a negative correlation between the U.S. dollar index and metal prices, but positive relationship between the U.S. dollar index and the overall level of foreign supply, so the expected sign of the coefficient estimate of this variable is positive in the supply side equation. The dollar index is calculated by a geometric weighted average of the change in the major seven foreign currency (Canadian dollar-CAD, Euro-EUR, Japanese yen-JPY, British pound-GBP, Swiss franc-CHF, Australian dollar-AUD, \& Swedish kronor-SEK) exchange rates against the U.S. dollar relative to the base date of March $1973=100$ when the Smithsonian agreement was made. This agreement allowed the world's major trading nations' currencies to float freely against each other and it replaced the fixed rate Bretton Woods agreement of New Hampshire (Sotowicz, June 2004, p. 28). The dollar index has traded as high as 144 in March 1985 and as low as 80 in April 1995, and is very volatile over time. A value of 144 means the dollar's value has risen $44 \%$ against the basket of major currencies since March 1973.

The last exogenous variable that is used to explain copper supply is the one-year (1Y) LIBOR (London inter-bank offering rate) interest rate. According to the Classical LongRun Economic Model, the interest rate is the reward for saving and supplying funds to 
the loanable funds market and the financial markets. The loanable fund market is where households make their saving available to those who need additional funds (Hall, Lieberman, 2001, p. 590). Hence, rise in the interest rate decreases household consumption, encourages consumers to save more money and increases household savings. Indeed, consumers spending and business spending are very sensitive to changes in the interest rate. On the other hand, investment depends on the interest rate because copper producers (generally businesses) need plant and equipment to carry out their operation to produce copper. They obtain the funds for their investment from the loanable funds market, so a key cost of any investment project is the interest rate that must be paid for on borrowed funds.

Figure (5.2): Supply of Funds

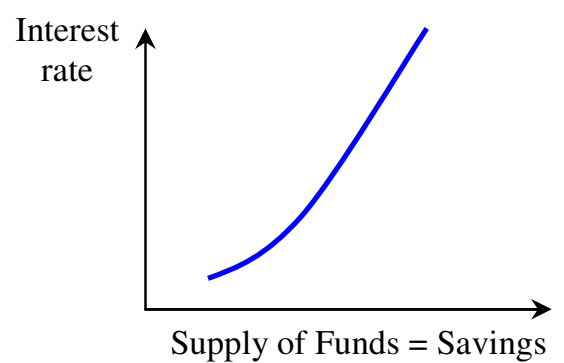

Figure (5.3): Investment Demand

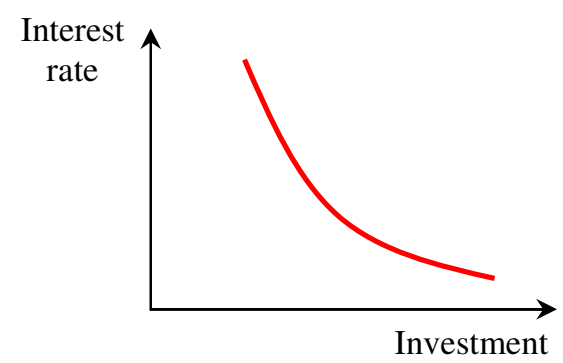

As the interest rate rises, the costs of investment increase, net investment decreases, fewer projects will be implemented, investment spending will decline, therefore, less copper will be produced eventually. By contrast, a lower interest rate stimulates consumers spending on durables and business spending on plant and equipment. Moreover, at low interest rates, more funds are demanded for investment projects. This is a long term effect on copper supply; hence, a long term lagged interest rate is a necessary variable in the supply equation. 
Most researchers and analysts recommend using the LIBOR interest rate as the appropriate rate of interest for econometric research because this is the key interest rate in the Eurocurrency market, which is the international deposit and loan market: LIBOR. Some large London banks act as brokers in the Eurocurrency market, where banks from around the world trade overnight funds. This market is extremely competitive because many banks participate. The LIBOR and the Fed funds are close substitutes.

The longest term LIBOR is the one-year rate. In addition, interest rate in a specific time period does not immediately affect the decisions of copper producers (i.e. copper supply) in that specific period of time. Therefore, the interest rate variable should be lagged. As mentioned before, the interest rate variable has a long term effect on copper supply, so the variable should be lagged by at least from 6 months to 12 months if there are monthly data used. In this analysis, lagging the interest rate variable by 6 months was statistically more significant than lagging the variable by 12 months. The outcomes of the two-stage least squares regressions are shown in tables A7-1 and A7-2 of appendix 7. If the interest rate variable is lagged by 12 months, then the essential exogenous explanatory variables in the supply equation including the lagged price of copper, time as a proxy for technology, the price of oil and the U.S. dollar index had statistically insignificant t-scores. Hence, the variable is lagged by 6 months (please compare table A2-3 with A7-2). The expected sign of the coefficient estimate of this interest rate variable in the structural equation of supply is negative.

The structural function of supply is specified with expected signs as follows:

$$
\left\{\begin{array}{l}
S_{t}=f\left\{P_{(t-1)}^{(+)}, T_{t}^{(+)}, P_{\text {OIL }(t)}^{(-)}, U_{S D I}^{(+)}, \operatorname{LIBOR}^{(-)}(1 Y)_{(t-6)}\right\} \\
S_{t}=\text { PRODUCTION }_{t}
\end{array}\right.
$$


According to the Cobweb model, the expected price of copper $\mathrm{P}_{\mathrm{t}}{ }^{*}$ is an essential variable for explaining copper supply as well as the price of the previous period $\mathrm{P}_{(\mathrm{t}-1)}$ is a good proxy for the expected price of copper $\mathrm{P}_{\mathrm{t}}{ }^{*}$. Therefore, $\mathrm{P}_{(\mathrm{t}-1)}=\mathrm{P}_{\mathrm{t}}{ }^{*}$ in the structural function of supply.

Table 5.2: Correlation Coefficients between Supply and its Exogenous Variables

\begin{tabular}{|c|c|c|c|c|c|}
\hline & $\mathbf{P}_{(\mathrm{t}-\mathbf{1})}$ & $\mathbf{T}_{\mathbf{t}}$ & $\mathbf{P}_{\text {OILt }}$ & USDI $_{\mathbf{t}}$ & LIBOR(1Y) $_{(\mathbf{t}-\mathbf{6})}$ \\
\hline $\mathbf{S}_{\mathbf{t}}$ & -0.4798 & 0.9301 & 0.6198 & 0.4949 & -0.6243 \\
\hline
\end{tabular}

The correlation coefficients above are calculated on the original data which have not been converted by any functional form such as log. As can be seen the table, the time variable has a strong relationship with the dependent variable of copper supply. In addition, other exogenous explanatory variables including the 6-month lagged LIBOR(1Y), the price of oil, the U.S. dollar index and the lagged price of copper have reasonable relationships with the dependent variable of copper supply.

\section{B) Choosing an Appropriate Functional Form:}

Figures (5.4) through (5.6) indicate the relationship between demand and its exogenous variables and (5.7) through (5.11) between supply and its exogenous variables. As shown in the figures, each exogenous variable is not linearly related to the endogenous variable. In addition, most econometric models use a double logarithmic functional form due to this non-linearity of variables. In a double log form, elasticity is constant but the slopes are not. Therefore, I used double log form in both structural equations of demand and supply. 
Figure (5.4):

World Cu Demand \& LME Cash Prices

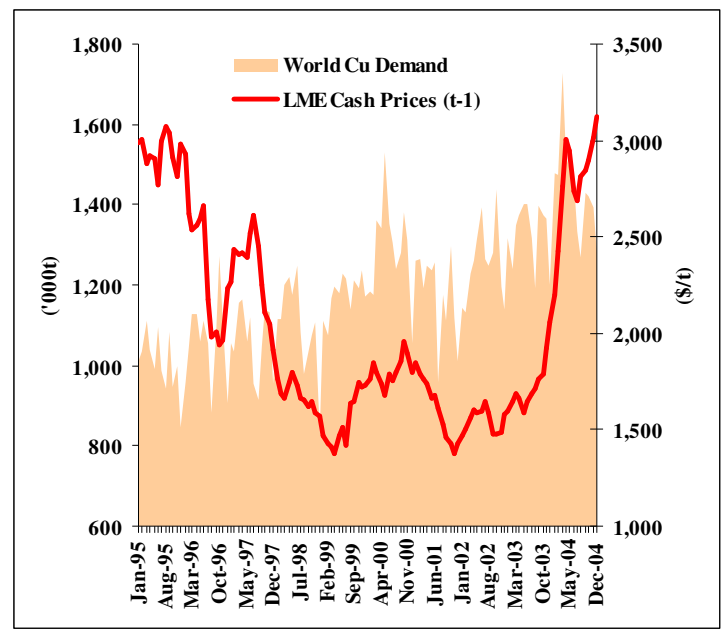

Figure (5.5):

World Cu Demand \& World GDP

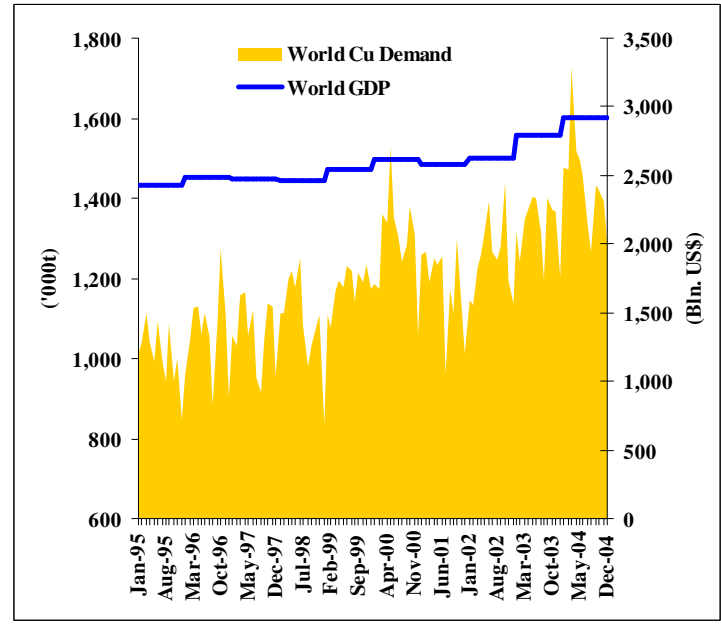

Figure (5.6):

World Cu Demand \& Global IP Growth

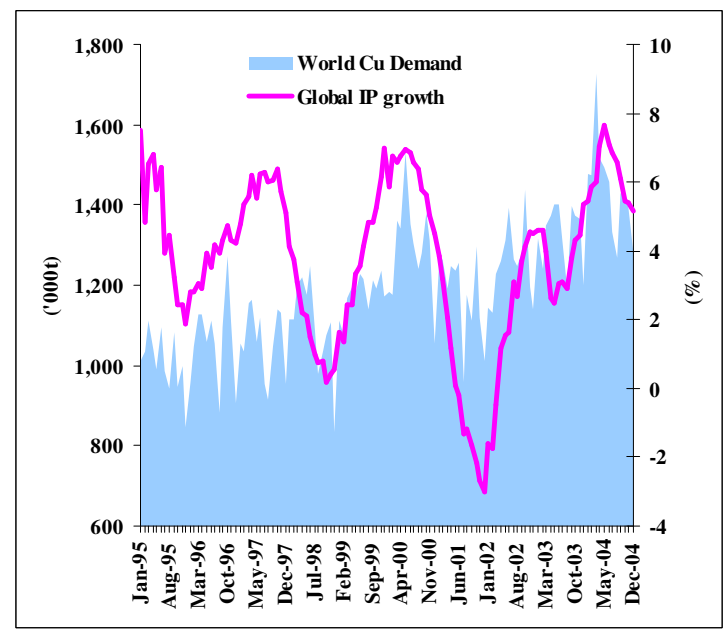

Figure (5.7):

World Cu Supply \& LME Cash Prices

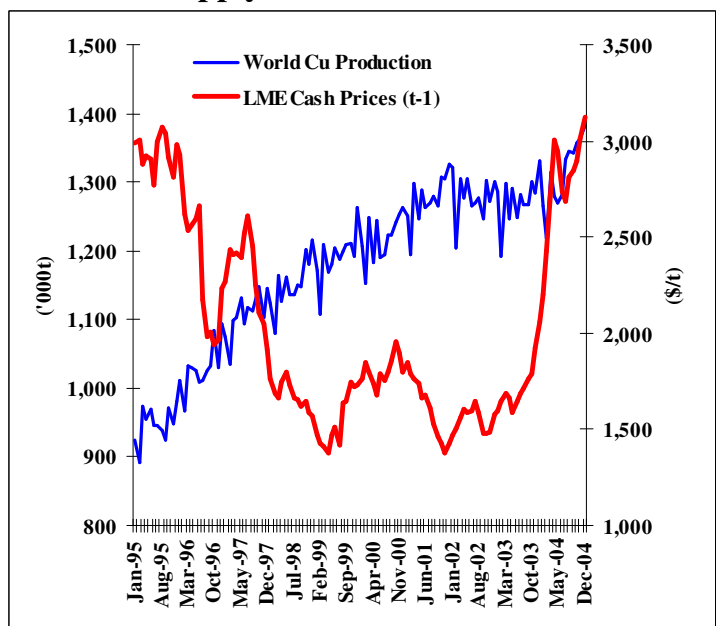

Figure (5.8):

World Cu Supply \& Time (Technology)

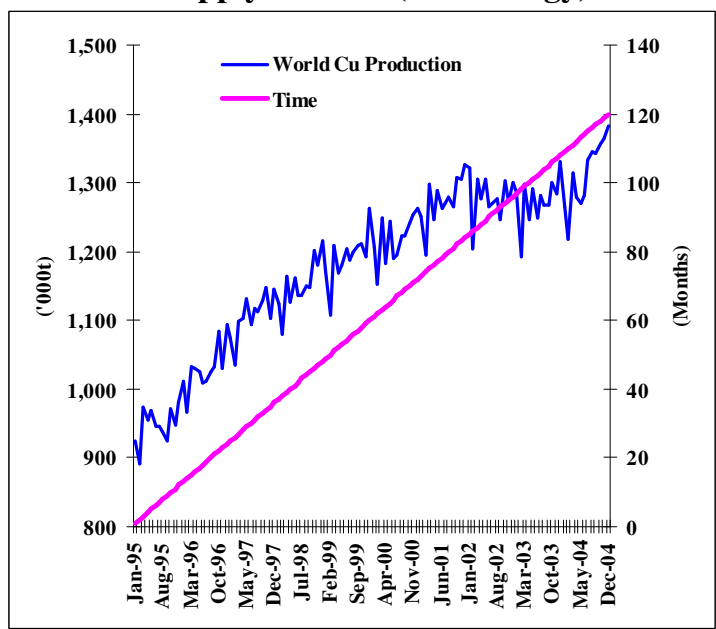

Figure (5.9):

World Cu Supply \& Price of Oil

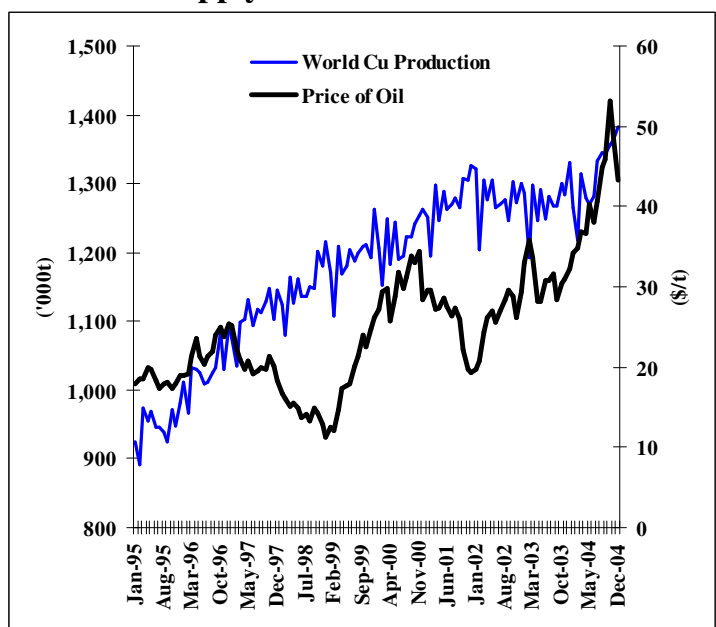


Figure (5.10):

World Cu Supply \& USD Index

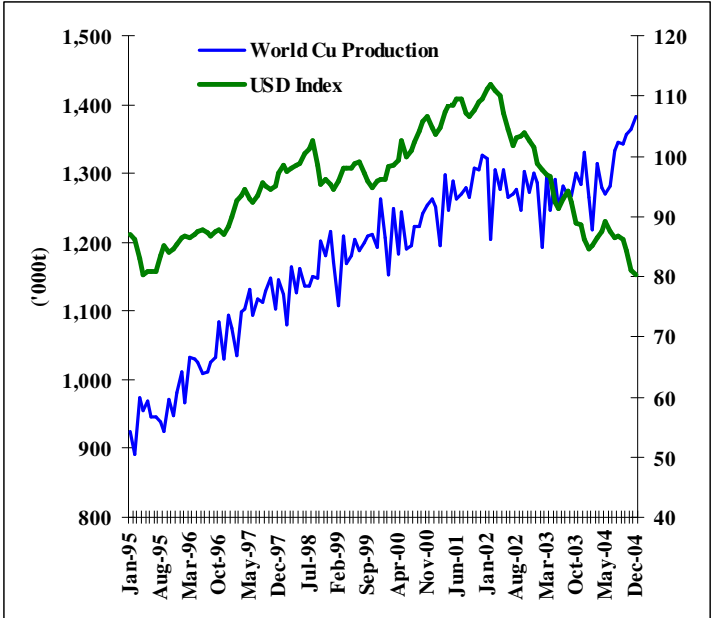

Figure (5.11):

World Cu Supply \& LIBOR (1Y) Rate

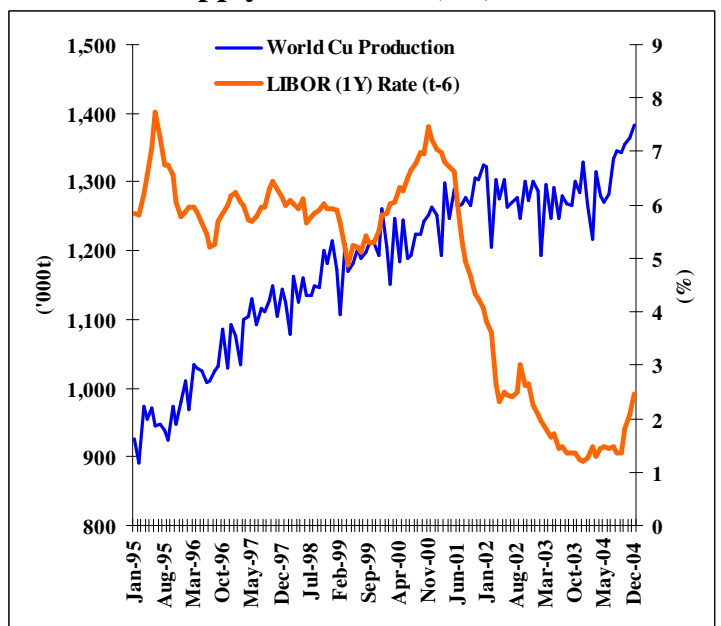

The linear function is only used for the Global IP growth variable because this variable can both be positive and negative and the log function can not be used with negative numbers. The mathematical model used is as follows:

\section{Mathematical Model:}

$$
\left\{\begin{array}{c}
L N\left(Q_{t}^{D}\right)=\alpha_{0}+\alpha_{1} \cdot L N\left(P_{(t-1)}\right)+\alpha_{2} \cdot L N\left(G D P_{t}\right)+\alpha_{3} \cdot I P_{t}+\varepsilon_{t} \\
L N\left(Q_{t}^{S}\right)=\beta_{0}+\beta_{1} \cdot L N\left(P_{(t-1)}\right)+\beta_{2} \cdot L N\left(T_{t}\right)+\beta_{3} \cdot L N\left(P_{\text {OILt }}\right)+ \\
+\beta_{4} \cdot L N\left(U S D I_{t}\right)+\beta_{5} \cdot L N\left(L I B O R_{(t-6)}\right)+u_{t} \\
Q_{t}^{D}=Q_{t}^{S}
\end{array}\right.
$$

$$
\begin{aligned}
& Q_{t}^{D}=e^{\alpha_{0}} \cdot P_{(t-1)}^{\alpha_{1}} \cdot G D P_{t}^{\alpha_{2}} \cdot e^{\alpha_{3} I P \%_{t}} \cdot e^{\mathcal{E}_{t}} \\
& \left\{Q_{t}^{D}=e^{\beta_{0}} \cdot P_{(t-1)}^{\beta_{1-1}} \cdot T_{t}^{\beta_{2}} \cdot P_{\text {olt }}^{\beta_{3}} \cdot U_{S D I} \beta_{t} \cdot \operatorname{LIBOR}_{(t-6)}^{\beta_{5}} \cdot e^{u_{t}}\right. \\
& Q_{t}^{D}=Q_{t}^{S}
\end{aligned}
$$




\subsection{HYPOTHESIS TESTING}

The null hypothesis is the result that we are trying to reject, so this is the opposite of our theory, which is the alternative hypothesis. The alternative hypothesis is the range of values of the coefficient estimates that are expected if our theory is correct.

Based upon the relevant theories, which were discussed in the previous section (5.1), the signs of the coefficients of all exogenous variables can be hypothesized. The table below summarizes hypothesis setting for each coefficient of the exogenous variables for both structural equations of demand and supply.

Table 5.3: The Expected Hypothesis

\begin{tabular}{|l|r|r|c|}
\hline $\begin{array}{c}\text { Exogenous } \\
\text { Variables }\end{array}$ & $\begin{array}{c}\text { Null Hypothesis: } \mathbf{H}_{\mathbf{0}} \\
\text { (what we hope to reject) }\end{array}$ & $\begin{array}{c}\text { Our Theory: } \mathbf{H}_{\mathbf{A}} \\
\text { (what we hope to find) }\end{array}$ & $\begin{array}{c}\text { Reject } \mathbf{H}_{\mathbf{0}} \\
\text { if: }\end{array}$ \\
\hline$\bullet \mathrm{P}_{(\mathrm{t}-1)}$ & $\alpha_{\mathrm{LN}[\mathrm{P}(\mathrm{t}-1)]} \geq 0$ & $\alpha_{\mathrm{LN}[\mathrm{P}(\mathrm{t}-1)]}<0$ & $\left|\mathrm{t}_{\mathrm{LN}[\mathrm{P}(\mathrm{t}-1)]}\right|>\mathrm{t}_{\mathrm{c}} \& \mathrm{t}_{\mathrm{LN}[\mathrm{P}(\mathrm{t}-1)]}(-)$ \\
\hline$\bullet \mathrm{GDP}_{(\mathrm{t})}$ & $\alpha_{\mathrm{LN}[\mathrm{GDP}(\mathrm{t})]} \leq 0$ & $\alpha_{\mathrm{LN}[\mathrm{GDP}(\mathrm{t})]}>0$ & $\left|\mathrm{t}_{\mathrm{LNGDP}(\mathrm{t})]}\right|>\mathrm{t}_{\mathrm{c}} \& \mathrm{t}_{\mathrm{LN}[\mathrm{GDP}(\mathrm{t})]}(+)$ \\
\hline$\bullet \mathrm{IP}_{(\mathrm{t})}$ & $\alpha_{\mathrm{IP} \%(\mathrm{t})} \leq 0$ & $\alpha_{\mathrm{IP} \%(\mathrm{t})}>0$ & $\left|\mathrm{t}_{\mathrm{IP} \%(\mathrm{t})}\right|>\mathrm{t}_{\mathrm{c}} \& \mathrm{t}_{\mathrm{IP} \%(\mathrm{t})}+(+)$ \\
\hline
\end{tabular}

\begin{tabular}{|c|c|c|c|}
\hline $\begin{array}{c}\text { Exogenous } \\
\text { Variables }\end{array}$ & $\begin{array}{c}\text { Null Hypothesis: } \mathbf{H}_{\mathbf{0}} \\
\text { (what we hope } \\
\text { to reject) }\end{array}$ & $\begin{array}{c}\text { Our Theory: } \mathbf{H}_{\mathbf{A}} \\
\text { (what we hope } \\
\text { to find) }\end{array}$ & $\begin{array}{l}\text { Reject } \mathbf{H}_{\mathbf{0}} \\
\text { if: }\end{array}$ \\
\hline - $\mathrm{P}_{(\mathrm{t}-1)}$ & $\beta_{\mathrm{LN}[\mathrm{P}(\mathrm{t}-1)]} \leq 0$ & $\beta_{\mathrm{LN}[\mathrm{P}(\mathrm{t}-1)]}>0$ & $\left|t_{\mathrm{LN}[\mathrm{P}(\mathrm{t}-1)]}\right|>\mathrm{t}_{\mathrm{c}} \& \mathrm{t}_{\mathrm{LN}[\mathrm{P}(\mathrm{t}-1)]}(+)$ \\
\hline$-\mathrm{T}_{(\mathrm{t})}$ & $\beta_{\mathrm{LN}[\mathrm{T}(\mathrm{t})]} \leq 0$ & $\beta_{\mathrm{LN}[\mathrm{T}(\mathrm{t})]}>0$ & $\left|t_{L N[T(t)]}\right|>t_{c} \& t$ \\
\hline - $\mathrm{P}_{\mathrm{OIL}(\mathrm{t})}$ & $\beta_{\mathrm{LN}[\mathrm{P}-\mathrm{OIL}(\mathrm{t})]} \geq 0$ & $\beta_{\mathrm{LN}[\mathrm{P}-\mathrm{OIL}(\mathrm{t})]}<0$ & $\left|t_{\text {LN[P-OIL(t)] }}\right|>t_{c} \& t_{\text {LN[P-OIL(t)] }}(-)$ \\
\hline - $\mathrm{USDI}_{(t)}$ & $\beta_{\mathrm{LN}[\mathrm{USDI}(t)]} \leq 0$ & $\beta_{\mathrm{LN}[\mathrm{USDI}(\mathrm{t})]}>0$ & $\left|t_{L N[U S D I(t)]}\right|>t_{c} \& t_{L N[U S D I(t)]}(+)$ \\
\hline - $\mathrm{LIBOR}_{(\mathrm{t}-6)}$ & $\beta_{\mathrm{LN}[\operatorname{LiBOR}(\mathrm{t}-6)]} \geq 0$ & $\beta_{\mathrm{LN}[\mathrm{LIBOR}(\mathrm{t}-6)]}<0$ & $\left|t_{\operatorname{LN}[\operatorname{LiBOR}(\mathrm{t}-6)]}\right|>\mathrm{t}_{\mathrm{c}} \& \mathrm{t}_{\mathrm{LN}[\operatorname{LIBOR}(\mathrm{t}-6)]}(-)$ \\
\hline
\end{tabular}




\subsection{DATA DESCRIPTION AND COLLECTION}

In the simultaneous equation model, all data consist of monthly time series between January 1995 and December 2004. The data was limited by January 1995 because prior to this date, monthly time series data of world copper production did not include net receipts, but that is included since January 1995 (Receipts $=$ Shipments - Production). For this reason, there was a huge jump in the data of world production before and after 1995. Therefore, the data for production of refined copper from 1995 are not comparable to the previous years. There are 120 months between January 1995 and December 2004, so 120 observations are used in the two-stage least square regression analysis.

The data for the demand for refined copper were obtained by summing copper usage and changes in total stocks of the world, as reported by ICSG. The data used for cash copper price is monthly time series data from the LME since January 1995. Copper is traded on three major exchanges in the world, including the LME, COMEX (branch of the NYMEX) and Shanghai Futures Exchange (SHFE). The biggest non-ferrous metal exchange is the LME, so the LME copper prices can represent the appropriate price for copper that is used in the analysis. The International Monetary Fund (IMF) reports annual world GDP data in its World Economic Outlook, and the data sums the GDP's of 176 countries. Monthly data on world GDP were not available because there was no organization that reports this data. Therefore, the annual data is divided by 12 in order to obtain monthly time series. CHR Metals Ltd. publishes monthly reports that contain yearover-year global industrial production (IP) growth. The data on the world refined copper production were obtained from the ICSG statistics as well as the annual bulletins "Metal Statistics", which are published by the American Metal Market Company. 
The price of West Texas intermediate crude oil is used as a representative price of oil in the analysis. These data were reported by the web page Economagic.com. The U.S. Federal Reserve reports the nominal major currencies dollar index in its statistical release on its official web page.

The historical data on 1-year LIBOR were obtained from the web site of the HSH Associates, Financial Publishers, which is the U.S. largest publisher of consumer loan information. Finally, the table below represents the units of measurement for each data series.

Table 5.4: The Units of Measurement for the Data

\begin{tabular}{|l|c|c|c|c|c|c|c|}
\hline & $\begin{array}{c}\text { ('000) } \\
\text { tonnes }\end{array}$ & $\begin{array}{c}\text { \$U.S. per } \\
\text { tonne }\end{array}$ & $\begin{array}{c}\text { \$U.S. per } \\
\text { barrel }\end{array}$ & $\begin{array}{c}\text { Billion } \\
\text { \$U.S. }\end{array}$ & $\begin{array}{c}\text { Percent } \\
\%\end{array}$ & Months & Index \\
\hline - Cu Demand & $\star$ & & & & & & \\
\hline - LME Cu Price & & $\star$ & & & & & \\
\hline - World GDP & & & & $\star$ & & & \\
\hline - Global IP growth & & & & & $\star$ & & \\
\hline - Cu Supply & $\star$ & & & & & & \\
\hline - Time & & & & & & $\star$ & \\
\hline - Price of Oil & & & $\star$ & & & & \\
\hline - U.S. Dollar Index & & & & & & & $\star$ \\
\hline - LIBOR (1Y) & & & & & $\star$ & & \\
\hline
\end{tabular}

Copper and oil prices and world GDP are in nominal (current) values because forward prices and strike prices of options contracts are in terms of nominal values. Therefore, forecast prices should be in current dollars in order to compare them to the prices on the forward and options quotes.

The equations were estimated by two-stage least squares (2SLS) in order to prevent biased and inconsistent parameter estimates due to the presence of endogenous explanatory variables. The analysis and estimated equations are in the following section. 


\subsection{TWO-STAGE LEAST SQUARES REGRESSION (2SLS) ANALYSIS}

This section presents the results of the simultaneous model estimation. As mentioned before, simultaneous equations violate Classical Assumption III, so the solution to the problem is to find a variable that is a good proxy for the endogenous variable and is uncorrelated with the error term. This proxy variable is called an instrumental variable, which replaces an endogenous variable that appears as explanatory variable on the right side of the equation. 2SLS analysis is a method of systematically creating this instrumental variable. The procedure for this analysis consists of two stages:

Stage 1: Run ordinary least squares (OLS) on the reduced-form equation for each of the endogenous variables that appears as explanatory variables in the structural equations of the model. From the system (5.9), the endogenous variable that appears as an explanatory variable is the lagged price of copper. Therefore, the dependent variable is the lagged price of copper $\mathrm{P}_{(\mathrm{t}-1)}$ and all predetermined variables are $\mathrm{GDP}_{(\mathrm{t})}, \operatorname{IP}_{(\mathrm{t})}, \mathrm{T}_{(\mathrm{t})}$, $\mathrm{P}_{\mathrm{OIL}(\mathrm{t})}, \mathrm{USDI}_{(\mathrm{t})}$ and $\mathrm{LIBOR}_{(\mathrm{t}-6)}$ in the reduced form equation. The results of the reducedform equation are as follows:

$$
\overline{\operatorname{Ln}\left[P_{(t-1)}\right]}=6.1455+1.0521 \cdot \operatorname{Ln}\left[G D P_{t}\right]-0.0009 \cdot I P_{t}-0.1291 \cdot \operatorname{Ln}\left[T_{t}\right]+
$$

Standard error: $\quad(0.7693) \quad(0.0062) \quad(0.0286)$

T-statistics: $\quad 1.3676 \quad-0.1387 \quad-4.5076$

$$
+0.2681 \cdot \operatorname{Ln}\left[P_{t}^{O I L}\right]-1.6069 \cdot \operatorname{Ln}\left[U S D I_{t}\right]+0.0950 \cdot \operatorname{Ln}\left[\operatorname{LIBOR}_{(t-6)}\right]
$$

Standard error: $\quad(0.0720) \quad(0.2278)$

T-statistics:

3.7248

$-7.0546$

$$
\mathrm{R}^{2}=0.78, \quad \bar{R}^{2}=0.77, \quad \mathrm{~F}=66.27, \quad \mathrm{n}=120
$$


There is no need to test any hypothesis on the reduced form equations.

Stage 2: Stage 2 is to substitute the reduced form dependent variables for the endogenous variables that appear only on the right side of the structural equations, then estimate these revised structural equations with OLS. In the revised structural equations, the dependent variables are still the original endogenous variables (demand and supply in our analysis) and that the substitutions are only for the endogenous variables where they appear on the right side of the structural equations (lagged price of copper in our analysis). Therefore, $\overline{\operatorname{Ln}\left[P_{(t-1)}\right]}$ from equation (5.10) is substituted into equation (5.9), so that the following results are obtained:

$\overline{\operatorname{Ln}\left[Q_{t}^{D}\right]}=-5.0485-0.1868 \cdot \operatorname{Ln}\left[P_{(t-1)}\right]+1.7151 \cdot \operatorname{Ln}\left[G D P_{t}\right]+0.0158 \cdot I P_{t}$

Standard error: $\quad(0.0436) \quad(0.1377) \quad(0.0040)$

$\begin{array}{lll}\text { T-statistics: } & -4.2809 & 12.4513\end{array}$

$$
\mathrm{R}^{2}=0.65, \quad \bar{R}^{2}=0.64, \quad \mathrm{~F}=70.56, \quad \mathrm{n}=120
$$

$\left.\overline{\operatorname{Ln}\left[Q_{t}^{S}\right.}\right]=-3.0785+0.5960 \cdot \operatorname{Ln}\left[P_{(t-1)}\right]+0.1408 \cdot \operatorname{Ln}\left[T_{t}\right]-0.1559 \cdot \operatorname{Ln}\left[P_{t}^{O I L}\right]+$

Standard error: $\quad(0.1952) \quad(0.0214) \quad(0.0655)$

$\begin{array}{llll}\text { T-statistics: } & 3.0537 & 6.5702 & -2.3808\end{array}$

$$
+1.2432 \cdot \operatorname{Ln}\left[U S D I_{t}\right]-0.0561 \cdot \operatorname{Ln}\left[\operatorname{LIBOR}_{(t-6)}\right]
$$

Standard error: (0.3358) (0.0129)

T-statistics: $\quad 3.7022 \quad-4.3583$

$$
\mathrm{R}^{2}=0.91, \quad \bar{R}^{2}=0.91, \quad \mathrm{~F}=235.24, \quad \mathrm{n}=120
$$


As can be seen from the results of the revised structural equations (5.11) and (5.12), the signs of the coefficient estimates correctly match their expectations in both structural equations. The adjusted degree of fit $\bar{R}^{2}$ is reasonable 0.64 in the structural equation of demand and is high 0.91 in the supply-side structural equation.

The t-distribution tests whether an estimated slope coefficient is significantly different from a hypothesized value. The t-statistic is computed as:

$$
t_{k}=\frac{\left(\hat{\beta}_{k}-\beta_{H_{0}}\right)}{S E\left(\hat{\beta}_{k}\right)}
$$

Where: $\hat{\beta}_{k}=$ estimated slope coefficient $\operatorname{SE}\left(\hat{\beta}_{k}\right)=$ estimated standard error and $\beta_{H_{0}}$ is the value expected in the null hypothesis.

The computed t-statistic $t_{k}$ is compared with the critical $t$-value $t_{c}$ with the desired level of significance (5\% in this analysis) for a one-sided test, and (n-k-1) degrees of freedom.

If $\left|t_{k}\right|>t_{c}$ and $t_{k}$ has the sign implied by the alternative hypothesis, then the null hypothesis will be rejected.

$$
\begin{aligned}
& \mathrm{H}_{0}: \beta_{k} \leq \beta_{H_{0}} \\
& \mathrm{H}_{\mathrm{A}}: \beta_{k}>\beta_{H_{0}}
\end{aligned}
$$

The degrees of freedom (D.F.) equal 116 and 114 in the structural equations of demand and supply respectively.

$$
\begin{aligned}
& \text { D.F. }(\text { demand })=\mathrm{n}-\mathrm{k}-1=120-3-1=116 \\
& \text { D.F. (supply) }=\mathrm{n}-\mathrm{k}-1=120-5-1=114
\end{aligned}
$$

where, $\mathrm{n}=$ the number of observations and $\mathrm{k}=$ the number of explanatory variables 
The t-scores of the coefficient estimates are all significant because the critical value of the t-distribution is 1.658 for one-sided test with $5 \%$ level of significance and all $\left|t_{k}\right|$ are greater than $t_{c}$, as well as the sign of $t_{k} s$ are the same as the sign of the coefficient implied by alternative hypothesis $\mathrm{H}_{\mathrm{A}}$. Therefore, t-statistics are significant in both structural equations. The F-test is a method of testing the overall significance of a regression equation and it is computed as:

$$
F=\frac{E S S / k}{R S S /(n-k-1)}=\frac{\sum(\hat{Y}-\bar{Y})^{2} / k}{\sum e_{i}^{2} /(n-k-1)}
$$

Where: $\quad$ ESS = explained sum of squares $\quad$ RSS = residual sum of squares

$$
\begin{aligned}
& \hat{Y}=\text { the estimated value of the dependent variable } \\
& \bar{Y}=\text { the mean value of the dependent variable } \\
& \mathrm{e}_{\mathrm{i}}=\text { the residuals } \quad \mathrm{n}=\text { the number of observations } \\
& \mathrm{k}=\text { the number of explanatory variables }
\end{aligned}
$$

The computed F-statistic $(F)$ is compared with the critical F-value $\left(F_{c}\right)$ with the desired level of significance (5\%) for a two-sided test, and (n-k-1) degrees of freedom for denominator and $\mathrm{k}$ degrees of freedom for numerator. If calculated $\mathrm{F}$ is greater than $\mathrm{F}_{\mathrm{c}}$ $\left(\mathrm{F}>\mathrm{F}_{\mathrm{c}}\right.$ ), then the null hypothesis will be rejected. The hypotheses in the demand and supply equations are as follows:

$$
\mathrm{H}_{0}(\text { Demand }): \beta_{L N[P(t-1)]}=\beta_{L N[G D P(t)]}=\beta_{I P(t)}=0
$$

$\mathrm{H}_{\mathrm{A}}$ (Demand): $\mathrm{H}_{0}$ is not true

$$
\mathrm{H}_{0}\left(\text { Supply): } \beta_{L N[P(t-1)]}=\beta_{L N[T(t)]}=\beta_{L N[P(O i l)(t)]}=\beta_{L N[U S D I(t)]}=\beta_{L N[L I B O R(t-6)]}=0\right.
$$

$\mathrm{H}_{\mathrm{A}}$ (Supply): $\mathrm{H}_{0}$ is not true 
F-statistics for the demand and the supply equations are calculated 70.56 and 235.24 respectively. The critical $\mathrm{F}$ values for the demand and supply equations are 2.68 and 2.29 respectively. Hence, we reject the null hypothesis and conclude that the overall fits of the supply and the demand equations are both significant.

Common and classical econometric problems with OLS, including multicollinearity, autocorrelation and heteroskedasticity are tested in the following sections for both structural equations.

\section{A) Multicollinearity (MC):}

Multicollinearity is a strong linear relationship between the exogenous variables in the model and it can significantly affect the coefficient estimates. Perfect multicollinearity is the violation of the classical assumption VI, which states that no independent variable is a perfect linear function of one or more other explanatory variables. Imperfect multicollinearity is a strong linear relationship between two or more explanatory variables. The consequence of multicollinearity is that it is difficult to accurately estimate the coefficients, but they will remain unbiased. Therefore, it is essential to test whether there is a multicollinearity or not. If there is imperfect multicollinearity, the following regression results will have these consequences:

- The coefficient estimates will not be estimated accurately;

- The standard errors of the coefficient estimates will increase for the collinear variables;

- The t-scores will be overstated on the collinear variables; 
- The degree of fit $\left(\mathrm{R}^{2}\right)$ will mostly be unaffected and the adjusted degree of fit $\left(\bar{R}^{2}\right)$ may decrease slightly;

- $\quad$ The F- statistics will be unaffected.

As can bee seen from equations (5.11) and (5.12), the low standard errors and the significant t-statistics suggest that multicollinearity is not a problem in these structural equations. However, the correlation matrix between the explanatory variables and Variance Inflation Factor (VIF) are the important methods to test multicollinearity.

Table 5.5: Correlations between exogenous variables in the structural equation of demand

\begin{tabular}{lccc}
\hline & LN[P(t-1)] & LN[GDP(t)] & IP \%(t) \\
\hline LN[P(t-1)] & 1 & & \\
LN[GDP(t)] & 0.0002 & 1 & \\
IP \%(t) & 0.6022 & 0.1901 & 1 \\
\hline
\end{tabular}

Table 5.6: Correlations between exogenous variables in the structural equation of supply

\begin{tabular}{lccccc}
\hline & LN[P(t-1)] & LN[T(t)] & LN[P Oil(t)] & LN[USDI(t)] & LN[LIBOR $(\mathrm{t}-6)]$ \\
\hline LN[P(t-1)] & 1 & & & & \\
LN[T(t)] & -0.6135 & 1 & & & \\
LN[P Oil (t)] & 0.1892 & 0.5426 & 1 & & \\
LN[USDI(t)] & -0.9179 & 0.5046 & -0.0534 & 1 & \\
LN[LIBOR(t-6)] & -0.0074 & -0.5784 & -0.6187 & 0.1522 & 1 \\
\hline
\end{tabular}

The t-test is used to test if a simple correlation coefficient (r) between two explanatory variables is statistically significant.

$$
\begin{array}{ll}
\text { Hypothesis: } & \mathrm{H}_{0}: \mathrm{r}=0 \text { (there is no collinearity) } \\
& \mathrm{H}_{\mathrm{A}}: \mathrm{r} \neq 0 \text { (there is collinearity) }
\end{array}
$$

The null hypothesis of no correlation between two variables can be tested with: 


$$
\mathrm{t}_{\mathrm{r}}=\frac{\mathrm{r}_{1,2} \cdot \sqrt{(\mathrm{n}-2)}}{\sqrt{\left(1-\mathrm{r}_{1,2}^{2}\right)}}
$$

where: $\mathrm{n}=$ the number of observations, $\quad \mathrm{r}_{1,2}=$ correlation coefficient

The calculated $t_{r}$ is compared against the appropriate critical t-statistics $\left(t_{c}\right)$ with $(n-2)$ degrees of freedom. The tables below show the calculated t-statistics of the correlation coefficients.

Table 5.7: T-tests $\left(t_{r}\right)$ on the Structural Equation of Demand

\begin{tabular}{lccc}
\hline & $\mathrm{LN}[\mathrm{P}(\mathrm{t}-1)]$ & $\mathrm{LN}[\mathrm{GDP}(\mathrm{t})]$ & $\mathrm{IP} \%(\mathrm{t})$ \\
\hline $\mathrm{LN}[\mathrm{P}(\mathrm{t}-1)]$ & 1 & & \\
$\mathrm{LN}[\mathrm{GDP}(\mathrm{t})]$ & 0.0017 & 1 & \\
$\mathrm{IP} \%(\mathrm{t})$ & 8.1931 & 2.1034 & 1 \\
\hline
\end{tabular}

Table 5.8: T-tests $\left(t_{r}\right)$ on the Structural Equation of Supply

\begin{tabular}{lccccc}
\hline & LN[P(t-1)] & LN[T $(\mathrm{t})]$ & LN[P Oil $(\mathrm{t})]$ & LN[USDI(t)] & LN[LIBOR(t-6)] \\
\hline LN[P(t-1)] & 1 & & & & \\
LN[T(t)] & -8.4384 & 1 & & & \\
LN[P Oil(t)] & 2.0929 & 7.0172 & 1 & & \\
LN[USDI(t)] & -25.1312 & 6.3488 & -0.5805 & 1 & \\
LN[LIBOR(t-6)] & -0.0799 & -7.7019 & -8.5550 & 1.6729 & 1 \\
\hline
\end{tabular}

The critical value of t-statistics $\left(\mathrm{t}_{\mathrm{c}}\right)$ is 1.98 for two-tailed test with $5 \%$ level of significance and 118 degrees of freedom. Therefore, the t-test of the correlation coefficients concludes that correlations exist (i.e. $\left|t_{r}\right|>t_{c}=1.98$ ) between following explanatory variables:

$\operatorname{Ln}[\mathrm{P}(\mathrm{t}-1)]$ and IP\%(t) $\quad \operatorname{Ln}[\mathrm{P}(\mathrm{t}-1)]$ and $\operatorname{Ln}[\mathrm{P} . \mathrm{Oil}(\mathrm{t})] \quad \operatorname{Ln}[\mathrm{P}(\mathrm{t}-1)]$ and $\operatorname{Ln}[\mathrm{USDI}(\mathrm{t})]$

$\operatorname{Ln}[\mathrm{P}(\mathrm{t}-1)]$ and $\operatorname{Ln}[\mathrm{T}(\mathrm{t})] \quad \operatorname{Ln}[\mathrm{T}(\mathrm{t})]$ and $\operatorname{Ln}[\mathrm{P} . \mathrm{Oil}(\mathrm{t})] \quad \operatorname{Ln}[\mathrm{T}(\mathrm{t})]$ and $\operatorname{Ln}[\mathrm{USDI}(\mathrm{t})]$

$\operatorname{Ln}[G D P(t)]$ and IP\%(t) $\operatorname{Ln}[T(t)]$ and $\operatorname{Ln}[\operatorname{LIBOR}(t-6)] \operatorname{Ln}[P$. Oil $(t)]$ and $\operatorname{Ln}[\operatorname{LIBOR}(t-6)]$ 
However, this test works only for a pair of variables, so it does not look at more than pair wise multicollinearity.

\section{Variance Inflation Factor (VIF) test:}

VIF is computed by the $1 /\left(1-\mathrm{R}^{2}\right)$ ratio, in which $\mathrm{R}^{2}$ is the $\mathrm{R}$ squared of each subsidiary regression. According to the rule of thumb, if VIF is greater than five, severe multicollinearity may exist. Nevertheless, multicollinearity cannot be proved or disproved by VIF test because the test is based upon the rule of thumb, so theory is the most important guide.

Table 5.9: VIF Tests

\begin{tabular}{|l|c|c|c|}
\hline \multicolumn{5}{|c|}{ Structural equation of demand } \\
\hline Dependent Variable & $\mathbf{R}^{\mathbf{2}}$ & VIF $=\left[\mathbf{1} /\left(\mathbf{1}-\mathbf{R}^{\mathbf{2}}\right)\right]$ & Severe Multicollinearity \\
\hline $\mathrm{Ln}[\mathrm{P}(\mathrm{t}-1)]$ & 0.3762 & 1.60 & Does not Exist \\
\hline $\mathrm{IP} \%(\mathrm{t})$ & 0.3987 & 1.66 & Does not Exist \\
\hline $\mathrm{Ln}[\mathrm{GDP}(\mathrm{t})]$ & 0.0566 & 1.06 & Does not Exist \\
\hline \multicolumn{4}{|c|}{ Structural equation of supply } \\
\hline $\mathrm{Ln}[\mathrm{P}(\mathrm{t}-1)]$ & 0.9953 & 211.04 & May Exist \\
\hline $\mathrm{Ln}[\mathrm{T}(\mathrm{t})]$ & 0.9787 & 46.89 & May Exist \\
\hline $\mathrm{Ln}[\mathrm{P} . \mathrm{Oil}(\mathrm{t})]$ & 0.9802 & 50.44 & May Exist \\
\hline $\mathrm{Ln}[\mathrm{USDI}(\mathrm{t})]$ & 0.9901 & 101.22 & May Exist \\
\hline $\mathrm{Ln}[\mathrm{LIBOR}(\mathrm{t}-6)]$ & 0.8419 & 6.33 & May Exist \\
\hline
\end{tabular}

As a result, the VIF tests indicate that multicollinearity does not exist in the structural equation of demand, but exists in the structural equation of supply.

However, the VIF test has no perfect decision rule because it is a rule of thumb. In addition, it is possible to have multicollinear effects in an equation, which has no large VIFs. As a consequence, the VIF test is a sufficient but not necessary test for multicollinearity (Studenmund, 2001, p. 258). 
Theoretically, the lagged price of copper, time, price of oil and the U.S. dollar index variables are essential and should be in the equation for explaining copper supply. On the other hand, the multicollinearity in these variables may cause a specification bias. There is a high correlation between the lagged price of oil and the U.S. dollar index. If the U.S. dollar index variable is assumed to be an irrelevant variable and thus excluded from the system, the following results are obtained:

$\overline{\operatorname{Ln}\left[Q_{t}^{D}\right]}=-5.4079-0.1541 \cdot \operatorname{Ln}\left[P_{(t-1)}\right]+1.7302 \cdot \operatorname{Ln}\left[G D P_{t}\right]+0.0140 \cdot I P_{t}$

Standard error: $\quad(0.0505) \quad(0.1430) \quad(0.0043)$

$\begin{array}{lll}\text { T-statistics: } & -3.0497 & 3.2592\end{array}$

$$
\mathrm{R}^{2}=0.62, \quad \bar{R}^{2}=0.61, \quad \mathrm{~F}=63.22, \quad \mathrm{n}=120
$$

$\overline{\operatorname{Ln}\left[Q_{t}^{S}\right]}=6.7713-0.0212 \cdot \operatorname{Ln}\left[P_{(t-1)}\right]+0.0931 \cdot \operatorname{Ln}\left[T_{t}\right]+$

Standard error: (0.0539) (0.0146)

T-statistics: $\quad-0.3930 \quad 6.3945$

$$
+0.0356 \cdot \operatorname{Ln}\left[P_{t}^{O I L}\right]-0.0082 \cdot \operatorname{Ln}\left[\operatorname{LIBOR}_{(t-6)}\right]
$$

Standard error: $\quad(0.0270) \quad(0.0094)$

$\begin{array}{lll}\text { T-statistics: } & 1.3172 & -0.8662\end{array}$

$$
\mathrm{R}^{2}=0.89, \quad \bar{R}^{2}=0.89, \quad \mathrm{~F}=230.38, \quad \mathrm{n}=120
$$

If a multicollinearity is detected by removing the U.S. dollar index variable from the supply equation, standard errors of the estimated coefficients have to be reduced, t-scores have to be increased and $\bar{R}^{2}$ has to be increased slightly. 
As can be compared above results with the equations (5.11) and (5.12) though, standard errors are increased slightly, t-scores are decreased and $\bar{R}^{2}$ is decreased in the

demand equation. In the structural equation of supply, $\bar{R}^{2}$ is decreased, standard errors are decreased slightly and t-scores are also decreased due to the significant changes on the coefficient estimates. The lagged price of copper, the price of oil, the 6-month lagged interest rate variables have insignificant t-scores. In addition, the lagged price of copper and the price of oil variables had unexpected (wrong) signs. All these results were caused by omitting a relevant variable (U.S. dollar index) and the results were not met the requirements of detecting the multicollinearity. Although there is some evidence of multicollinearity, but it does not seem to be cause a problem in the equation. Therefore, not trying to correct the multicollinearity is best in the structural equation of supply.

\section{B) Autocorrelation (AC):}

Serial correlation or autocorrelation is a violation of Classical Assumption IV of OLS, which states that the observations of the error term are not correlated with each other. If there is any sort of pattern to the residuals, autocorrelation may exist.

According to following figures, there are no visible patterns to the distribution of the residuals. Therefore, autocorrelation is not likely a problem for both the structural equations of demand and supply, according to this initial test. 
Figure (5.12):

Distribution of the Residuals (Demand)

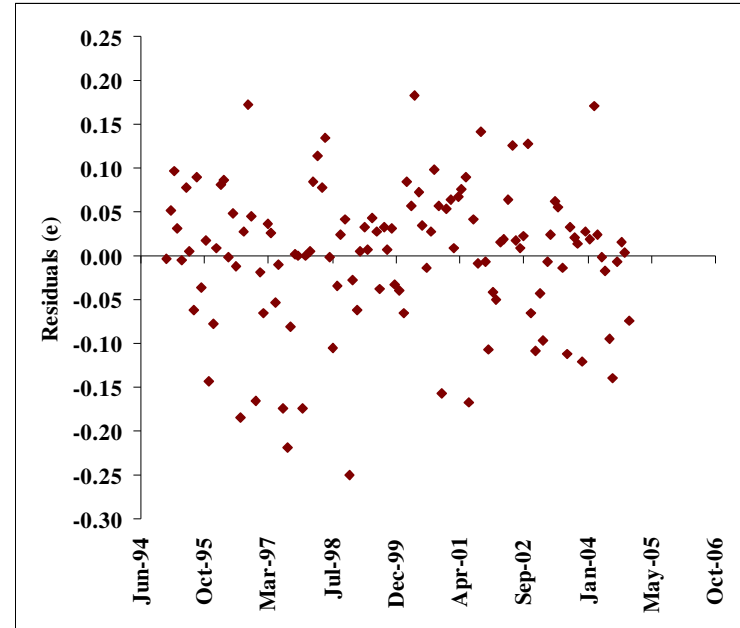

Figure (5.13):

Distribution of the Residuals (Supply)

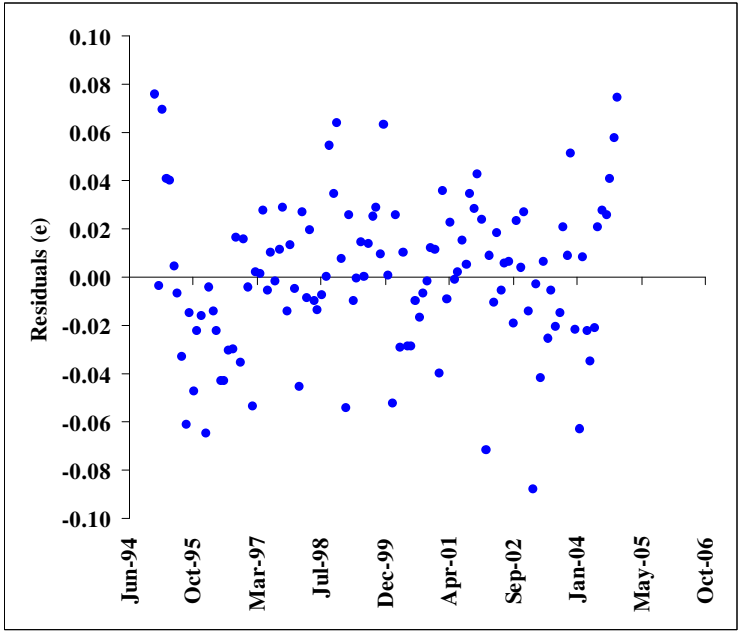

The Durbin-Watson (DW) d-statistic is used to test first-order autocorrelation in the error terms of an equation. The DW test works under the following assumptions:

a) The regression model includes an intercept term;

b) The serial correlation is first order;

c) There are no lagged dependent variables used as explanatory variables.

$\mathrm{P}_{(\mathrm{t}-1)}$ is a lagged dependent variable in the system model (5.9), but is an explanatory variable in each structural equation. Since d-statistic is testes in each structural equation, $\mathrm{P}_{(\mathrm{t}-1)}$ is not a lagged dependent variable in the structural equations of the supply and the demand. Therefore, all these assumptions are met in either supply or demand structural equations, so the DW statistic should be determined to test whether there is a first order autocorrelation or not.

The equation for the DW d-statistic for $(t)$ observations is: 


$$
\mathrm{d}_{\mathrm{DW}}=\frac{\sum_{\mathrm{t}=2}^{\mathrm{t}}\left(\mathrm{e}_{\mathrm{t}}-\mathrm{e}_{\mathrm{t}-1}\right)^{2}}{\sum_{\mathrm{t}=1}^{\mathrm{t}} \mathrm{e}_{\mathrm{t}}^{2}}
$$

where the $e_{t}$ are the OLS residuals.

The serial correlation is assumed to be first order in nature:

$$
\varepsilon_{\mathrm{t}}=\rho \cdot \varepsilon_{\mathrm{t}-1}+\mathrm{u}_{\mathrm{t}}
$$

where $\rho$ is the coefficient of serial correlation and $\mathrm{u}$ is a classical error term.

The hypothesis and appropriate decision rule are as follows:

$$
\begin{aligned}
& \mathrm{H}_{0}: \rho=0 \text { (no serial correlation) } \\
& \mathrm{H}_{\mathrm{A}}: \rho \neq 0 \text { (serial correlation) }
\end{aligned}
$$

$$
\begin{aligned}
& \text { If: } \quad \mathrm{d}<\mathrm{d}_{\mathrm{L}} \quad \text { Reject } \mathrm{H}_{0} \quad \text { (positive autocorrelation) } \\
& \text { If: } \quad d>4-d_{L} \quad \text { Reject } H_{0} \quad \text { (negative autocorrelation) } \\
& \text { If: } 4-\mathrm{d}_{\mathrm{U}}>\mathrm{d}>\mathrm{d}_{\mathrm{U}} \quad \text { Do not reject } \mathrm{H}_{0} \quad \text { (no autocorrelation) }
\end{aligned}
$$

Otherwise Inconclusive

Durbin-Watson statistic $\left(\mathrm{d}_{\mathrm{DW}}\right)$ is estimated as 1.5640 and 1.4615 for the structural equation of demand and supply respectively.

Table 5.10: Critical d-Values

\begin{tabular}{|l|c|c|c|c|c|c|c|}
\hline $\begin{array}{c}\text { Structural } \\
\text { equation }\end{array}$ & $\begin{array}{c}\text { Level of } \\
\text { significance } \\
\text { (two-sided) }\end{array}$ & $\begin{array}{c}\text { Number of } \\
\text { explanatory } \\
\text { variables }\end{array}$ & $\begin{array}{c}\text { Number of } \\
\text { data } \\
\text { observations }\end{array}$ & $\mathrm{d}_{\mathrm{L}}$ & $\mathrm{d}_{\mathrm{U}}$ & $4-\mathrm{d}_{\mathrm{U}}$ & $4-\mathrm{d}_{\mathrm{L}}$ \\
\hline Demand & $2 \%$ & 3 & 120 & 1.48 & 1.60 & 2.40 & 2.52 \\
\hline Supply & $2 \%$ & 5 & 120 & 1.44 & 1.65 & 2.35 & 2.56 \\
\hline
\end{tabular}


Figure (5.14): Testing $A C\left(\operatorname{Ln}\left[Q_{t}^{D}\right]\right)$
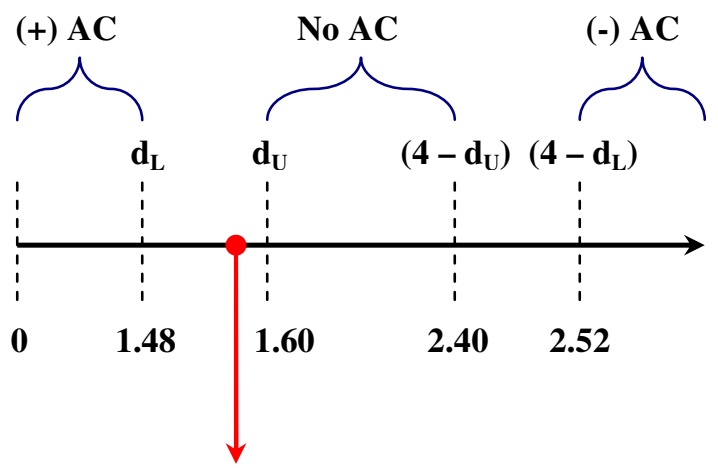

1.5647, so Inconclusive
Figure (5.15): Testing $A C\left(\operatorname{Ln}\left[Q_{t}{ }^{S}\right]\right)$

(+) $\mathrm{AC}$
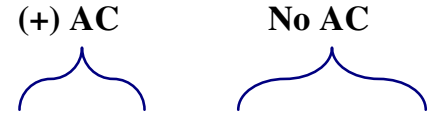

$\left(4-d_{U}\right)\left(4-d_{L}\right)$

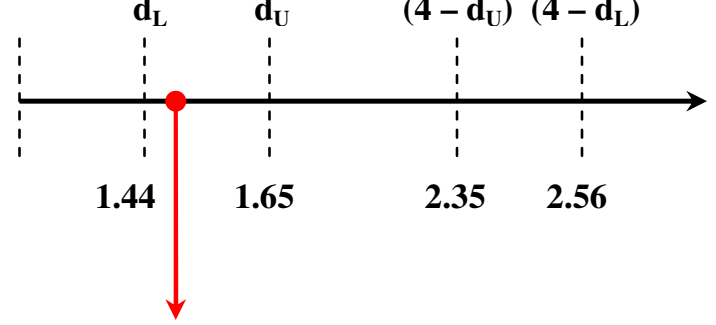

1.4734, so Inconclusive

The results are inconclusive, so it is impossible to tell whether autocorrelation exists or not in these structural equations. Rho $(\rho)$ is the parameter depicting the functional relationship between observations of the error term. The following equations are regression results which estimate the coefficients of serial correlation $\rho$ 's .

Structural equation of demand $(D)$ :

$$
e_{t}(D)=-0.0001+0.2157 \cdot e_{(t-1)}
$$

SE:

(0.0906)

T-stats:

2.3802

0.2157
Structural equation of supply $(S)$ :

$e_{t}(S)=-0.0005+0.2253 \cdot e_{(t-1)}$

SE:

$(0.0900)$

(5.20)

T-stats:

2.5035

$\operatorname{Rho}(\mathrm{S})$

0.2253

The coefficients of serial correlations $\rho$ 's are estimated as 0.2157 and 0.2253 in the structural equations of demand and supply respectively, which close to zero, but they are significantly different from zero; hence, there is some sign of autocorrelation. However, autocorrelation is not indicated by the DW d-statistics and there are no visible patterns to the distribution of the residuals, so autocorrelation is not likely a problem in this model. 


\section{C) Heteroskedasticity (HS):}

Heteroskedasticity is a violation of Classical Assumption VI, which says that the error term has a constant variance for all observations and it occurs very commonly in cross sectional data, but it may also occur in time series data.

In order to test for heteroskedasticity, plotting the residuals (e) against the various explanatory variables and looking for possible patterns is an initial test.

Figure (5.16): $\{e(D)\}$ vs $\operatorname{Ln}[P(t-1)]$

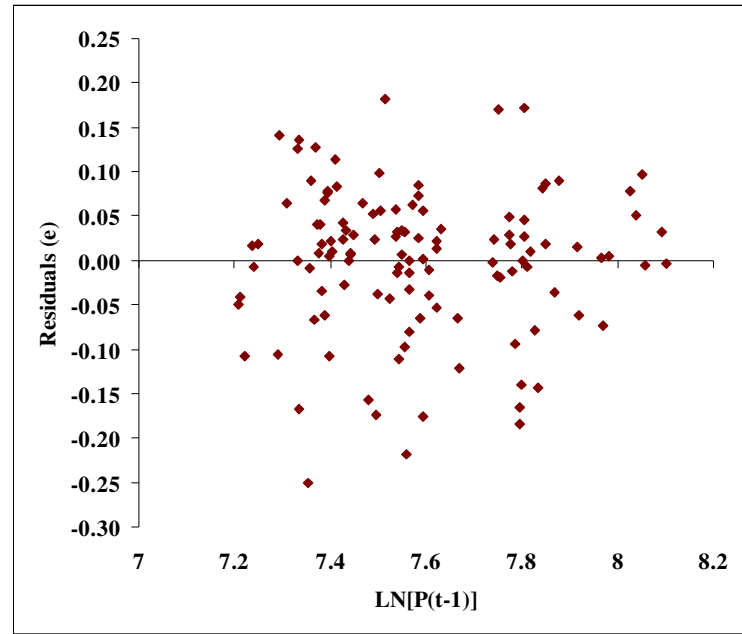

Figure (5.17): $\{e(D)\}$ vs Ln[GDP(t)]

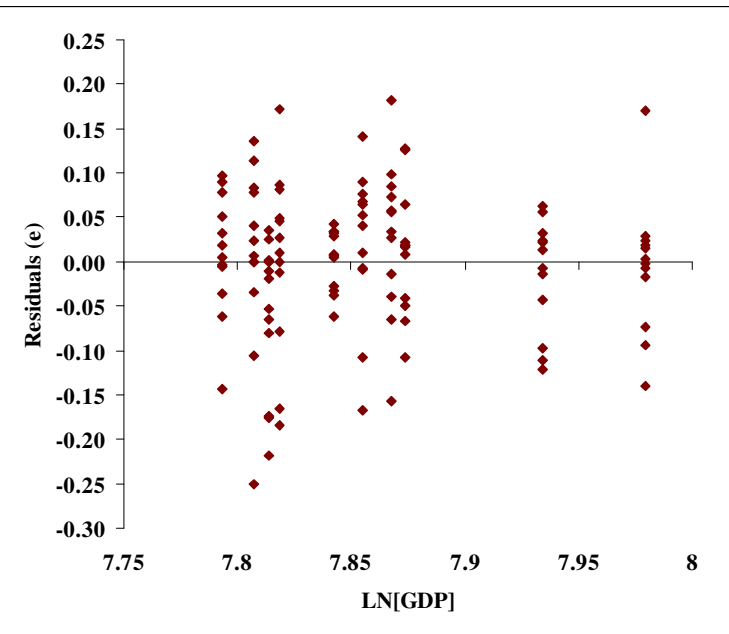

Figure (5.18): $\{e(D)\}$ vs $\operatorname{Ln}[\operatorname{IP} \%(t)]$

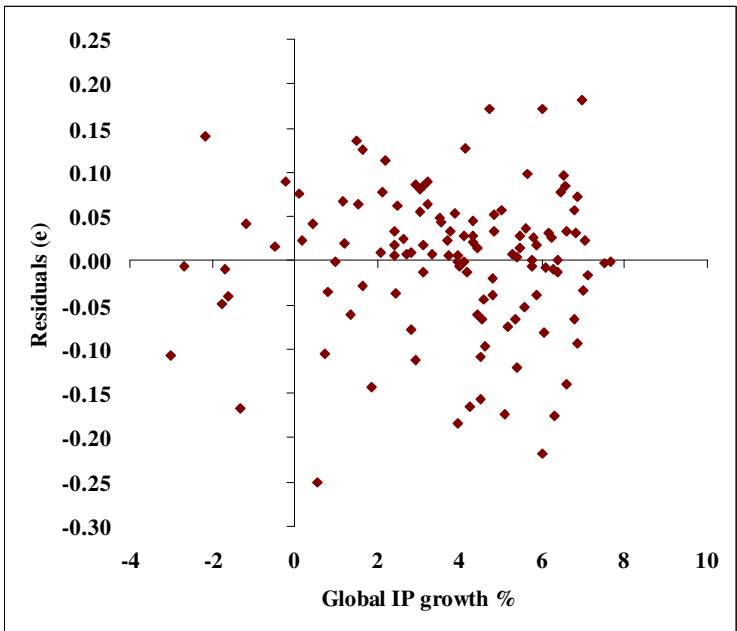

Figure (5.19): $\{\mathrm{e}(\mathrm{S})\}$ vs $\operatorname{Ln}[\mathrm{P}(\mathrm{t}-1)]$

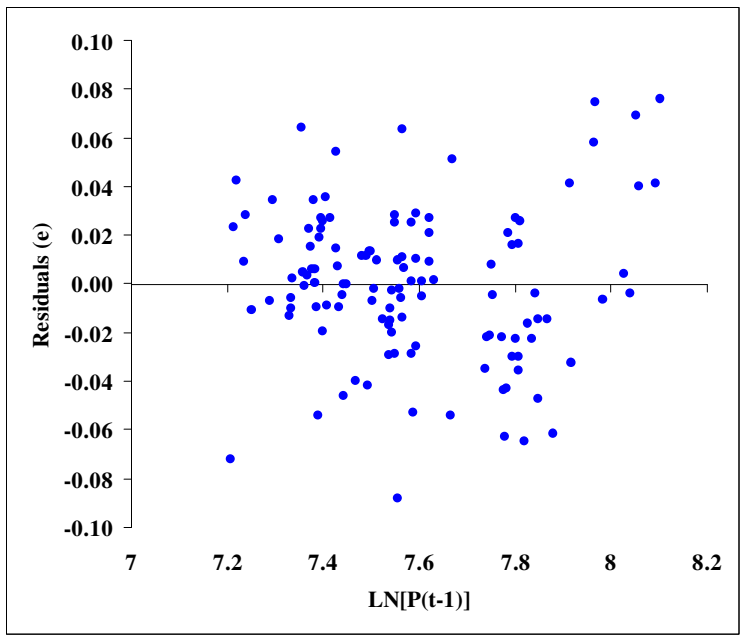


Figure (5.20): $\{\mathrm{e}(\mathrm{S})\}$ vs $\operatorname{Ln}[\mathrm{T}(\mathrm{t})]$

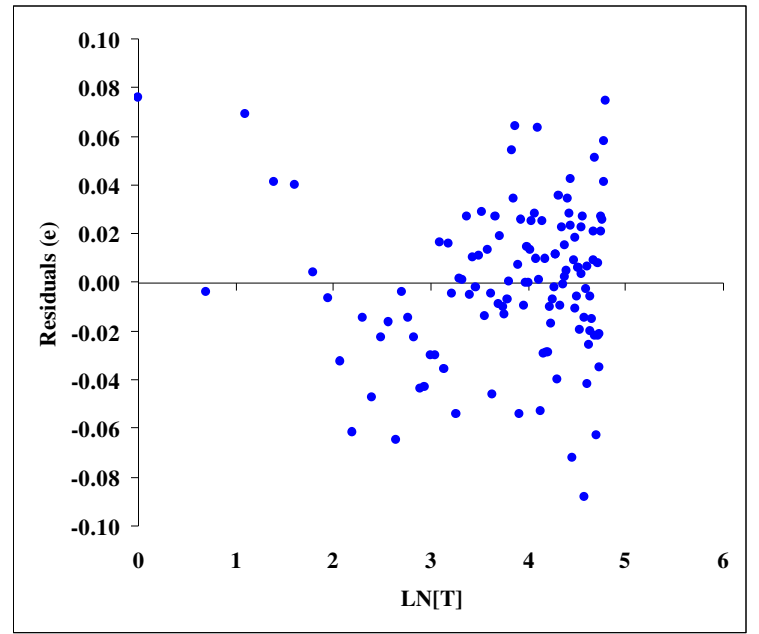

Figure (5.21): $\{e(S)\}$ vs $\operatorname{Ln}[$ P. Oil (t) $]$

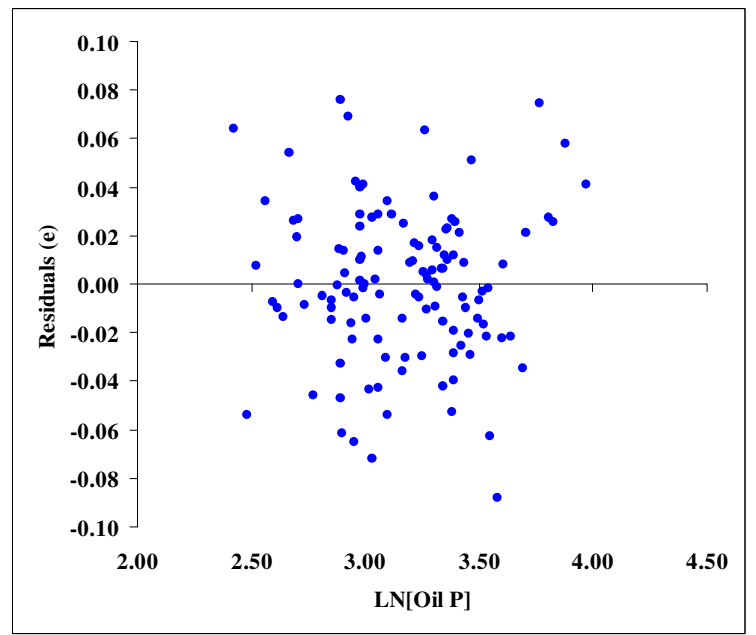

Figure (5.22): $\{\mathrm{e}(\mathrm{S})\}$ vs $\operatorname{Ln}[\mathrm{USDI}(\mathrm{t})]$

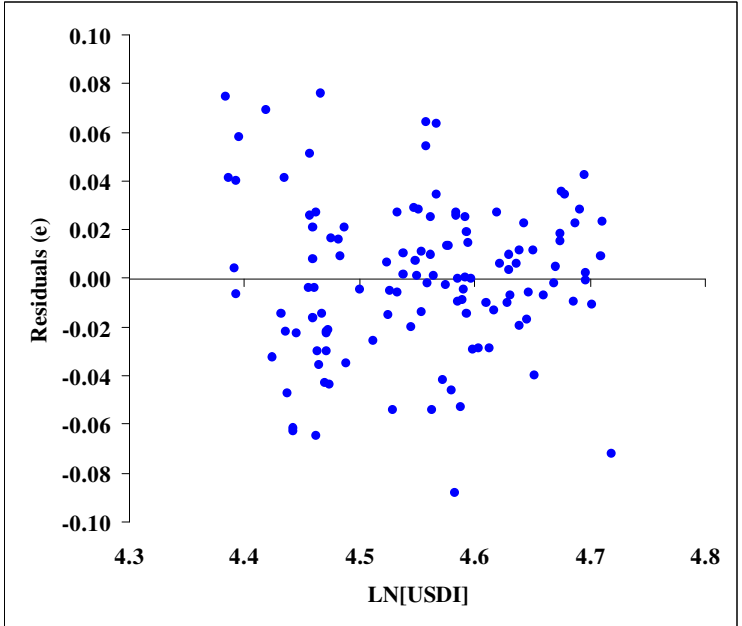

Figure (5.23): $\{e(S)\}$ vs Ln[LIBOR 1Y(t-6)]

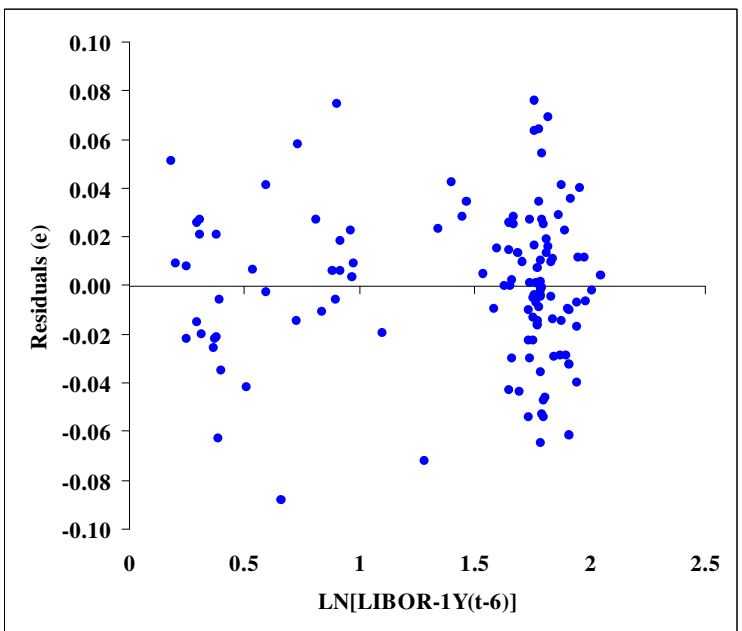

Figures (5.16) through (5.18) plot residual distributions of the structural equation of demand against its explanatory variables. The residuals are plotted against the explanatory variables of the supply-side equation in figures (5.19) through (5.23).

There are some sort of patterns on the figures (5.17), (5.20) and (5.23), so heteroskedasticity should be tested for by the Park and the White tests. 


\section{The Park Test}

This test is a procedure that attempts to test the residuals for heteroskedasticity and it has three steps. First, the residuals are obtained from the original regression. There are two structural equations including demand and supply, so two different residual distributions are obtained. The residuals are the difference between the actual and the predicted values.

$$
\mathrm{e}_{\mathrm{i}}=\mathrm{Y}_{\mathrm{i}}-\hat{\mathrm{Y}_{\mathrm{i}}}
$$

Second, the logarithmic function of squared errors is used as the dependent variable in a new regression where the explanatory variable is the log function of the proportionality factor $\mathrm{Z}$. The $\mathrm{Z}$ variable is called a proportionate variable.

$$
\operatorname{Ln}\left[e^{2}\right]=\alpha_{0}+\alpha_{1} \cdot \operatorname{Ln}[Z]+u
$$

where: $\mathrm{e}=$ the residual, $\mathrm{Z}=$ the possible proportionality factor,

$$
\mathrm{u}=\mathrm{a} \text { classical (homoskedastic) error term. }
$$

Finally, the results of the regression (5.23) are tested to see if there is any evidence of heteroskedasticity (Studenmund, 2001, p. 357). The hypothesis and appropriate decision rule are as follows:

$$
\begin{aligned}
& \mathrm{H}_{0}: \alpha_{1}=0 \quad \text { (homoskedasticity) } \\
& \mathrm{H}_{\mathrm{A}}: \alpha_{1} \neq 0 \quad \text { (heteroskedasticity) } \\
& \text { If }\left|\mathrm{t}_{\alpha_{1}}^{\mathrm{Z}}\right|>\mathrm{t}_{\mathrm{c}}, \quad \text { then reject } \mathrm{H}_{0}
\end{aligned}
$$


In the structural equation of demand, both the log function of lagged price of copper and GDP can be tested as proportionate variables in the structural equation of demand because the variance of the demand for copper will be high as the price of copper and GDP increase. The following equations report the regression results.

\section{$\underline{\operatorname{Ln}[\mathrm{P}(\mathrm{t}-1)] \text { as } \mathrm{Z} \text { variable: }}$}

$\operatorname{Ln}\left[e^{2}\right]=8.31-7.45 \cdot \operatorname{Ln}\left\{\operatorname{Ln}\left[P_{t-1}\right]\right\}$

SE:

t-stats:

$-0.87$
$\underline{\operatorname{Ln}[\mathrm{GDP}(\mathrm{t})] \text { as } \mathrm{Z} \text { variable: }}$

$\operatorname{Ln}\left[e^{2}\right]=3.43-4.96 \cdot \operatorname{Ln}\left\{\operatorname{Ln}\left[G D P_{t}\right]\right\}$

$-0.14$

The minimum critical t-value at $116(\mathrm{n}-\mathrm{k}-1=120-3-1)$ degrees of freedom and $5 \%$ level of significance with two-tailed test is 1.98 , which is not less than the calculated t-statistics in both cases (i.e. $\left|t_{\alpha_{1}}^{z}\right|<t_{c}$ ). Hence, we cannot reject $H_{0}$, which implies that homoskedasticity exists, so theoretically, heteroskedasticity is not likely a problem in the structural equation of demand.

In the structural equation of supply, choosing the $\log$ function of oil price or LIBOR(t-6) as a proportionality factor $\mathrm{Z}$, is more appropriate than selecting the other variables such as the price of copper, the U.S. dollar index, or the time (as a proxy for technological advance) because the key factor of any commodity production is the cost. Either price of oil or the interest rate is a cost side variable, so both can be useful as the $\mathrm{Z}$ variable. The critical $\mathrm{t}$-value at 114 degrees of freedom $(\mathrm{n}-\mathrm{k}-1=120-5-1)$ and $5 \%$ level of significance with two-tailed test is greater than 1.98 . 
$\underline{\mathrm{Ln}[\mathrm{P} . \text { Oil }(\mathrm{t})] \text { as Z variable: }}$

$\operatorname{Ln}\left[e^{2}\right]=-10.82+2.10 \cdot \operatorname{Ln}\left\{\operatorname{Ln}\left[P_{t}^{O I L}\right]\right\} \quad \operatorname{Ln}\left[e^{2}\right]=-8.32-0.46 \cdot \operatorname{Ln}\left\{\operatorname{Ln}\left[\operatorname{Libor}_{(t-6)}\right]\right\}$

SE:

t-stats:

0.95

(5.26)
$\underline{\operatorname{Ln}[\mathrm{LIBOR}-1 \mathrm{Y}(\mathrm{t}-6)] \text { as } \mathrm{Z} \text { variable: }}$
$-1.31$

In both cases, $\left|t_{\alpha_{1}}^{z}\right|<1.98<t_{c}$, so we cannot reject $H_{0}$. Therefore, heteroskedasticity may not exist in the structural equation of supply. In conclusion, heteroskedasticity is not likely to be a problem in the simultaneous system, according to the Park test.

\section{The White test}

In the White test, the squared residuals are used as the dependent variable. Unlike the Park test, the right-hand side of the secondary equation includes all the original explanatory variables, the squares of all the original explanatory variables, and the cross products of all the original explanatory variables with each other (Studenmund, 2001, p.361). The White test equation with three independent variables is as follows:

$$
\begin{aligned}
\left(\mathrm{e}_{\mathrm{i}}\right)^{2}= & \alpha_{0}+\alpha_{1} \cdot \mathrm{X}_{1 \mathrm{i}}+\alpha_{2} \cdot \mathrm{X}_{2 \mathrm{i}}+\alpha_{3} \cdot \mathrm{X}_{3 \mathrm{i}}+\alpha_{4} \cdot \mathrm{X}_{1 \mathrm{i}}^{2}+\alpha_{5} \cdot \mathrm{X}_{2 \mathrm{i}}^{2}+\alpha_{6} \cdot \mathrm{X}_{3 \mathrm{i}}^{2}+ \\
& +\alpha_{7} \cdot \mathrm{X}_{1 \mathrm{i}} \cdot \mathrm{X}_{2 \mathrm{i}}+\alpha_{8} \cdot \mathrm{X}_{1 \mathrm{i}} \cdot \mathrm{X}_{3 \mathrm{i}}+\alpha_{9} \cdot \mathrm{X}_{2 \mathrm{i}} \cdot \mathrm{X}_{3 \mathrm{i}}+\mathrm{u}_{\mathrm{i}}
\end{aligned}
$$

The appropriate decision rule is:

If $\left\{\mathrm{n} \cdot \mathrm{R}^{2}\right\}>\mathrm{Chi}-$ Squared statistics, then Reject $\mathrm{H}_{0}$ (homoskedasticity), and we conclude heteroskedasticity may exist.

Where: $\mathrm{n}=$ sample size,$\quad \mathrm{R}^{2}=$ unadjusted degree of fit 
The structural equation of demand contains three explanatory variables including the lagged price of copper, GDP and IP growth. The results of the White test regression are:

$$
\begin{gathered}
e_{i}^{2}=35.15-0.28 \cdot \operatorname{Ln}\left[P_{(t-1)}\right]-8.62 \cdot \operatorname{Ln}\left[G D P_{t}\right]+0.01 \cdot I P_{t}-0.01 \cdot\left\{\operatorname{Ln}\left[P_{(t-1)}\right]\right\}^{2}+ \\
\left.+0.52 \cdot\left\{\operatorname{Ln}\left[G D P_{t}\right]\right\}^{2}+0.0003 \cdot\left\{I P_{t}\right\}^{2}+0.05 \cdot \operatorname{Ln}\left[P_{(t-1)}\right] \cdot \operatorname{Ln}\left[G D P_{t}\right]-\quad \mathbf{5 . 2 9}\right) \\
-0.003 \cdot \operatorname{Ln}\left[P_{(t-1)}\right] \cdot I P_{t}+0.001 \cdot \operatorname{Ln}\left[G D P_{t}\right] \cdot I P_{t} \\
\mathrm{R}^{2}=0.0504, \quad \mathrm{n}=120, \quad\left\{\mathrm{n} \cdot \mathrm{R}^{2}\right\}=6.0508
\end{gathered}
$$

The degrees of freedom equal the number of slope coefficients in equation (5.29), which equals 9. The critical Chi-square value with 9 degrees of freedom and $5 \%$ level of significance is 16.92 , which larger $\operatorname{than}\left\{n \cdot R^{2}\right\}=6.05$. So, we cannot reject the null hypothesis of homoskedasticity, and conclude it is likely that we do not have a heteroskedasticity problem in the structural equation of demand.

There are five explanatory variables in the structural equation of supply, so the White test regression, in this case, contains 19 variables; however, the maximum number of independent variables is 16 in MS Excel, in order to run a regression (i.e. input X cannot contain more than 16 variables). Thus, heteroskedasticity cannot be tested in the structural equation of supply, using the White test.

In conclusion, heteroskedasticity is not a problem in both structural equations according to the Park and the White test results above. The evaluation of econometric problems including multicollinearity, autocorrelation, and heteroskedasticity indicates that the model should not be revised because these common econometric problems are 
not likely to be causing a severe problem in the simultaneous system. The result of the two-stage regression is:

$$
\left\{\begin{array}{l}
Q_{t}^{D}=e^{(-5.0485)} \cdot P_{(t-1)}^{(-0.1868)} \cdot G D P_{t}^{(1.7151)} \cdot e^{(0.0158) \cdot I P_{t}} \\
Q_{t}^{S}=e^{(-3.0785)} \cdot P_{(t-1)}^{(0.5960)} \cdot T_{t}^{(0.1408)} \cdot P_{O I L(t)}^{(-0.1559)} \cdot U S D I_{t}^{(1.2432)} \cdot \operatorname{LIBOR}_{(t-6)}^{(-0.0561)}
\end{array}\right.
$$

In a double-log equation, an individual regression coefficient (slopes $\beta_{\mathrm{k}} \mathrm{s}$ ) can be interpreted as an elasticity because:

$$
\beta_{\mathrm{k}}=\frac{\Delta(\ln \mathrm{Y})}{\Delta\left(\ln \mathrm{X}_{\mathrm{k}}\right)}=\frac{\Delta \mathrm{Y} / \mathrm{Y}}{\Delta \mathrm{X}_{\mathrm{k}} / \mathrm{X}_{\mathrm{k}}}=\text { Elasticity }_{\mathrm{Y}, \mathrm{X}_{\mathrm{k}}}
$$

The demand for copper is inelastic with respect to the lagged price and GDP (the indicator of the overall economic activity and income level), as shown by the average price-elasticity and income-elasticity of (0.1868) and 1.7151, respectively.

From the system equation (5.30), copper price can be estimated assuming quantity supplied $\mathrm{Q}_{\mathrm{t}}^{\mathrm{S}}$ equals quantity demanded $\mathrm{Q}^{\mathrm{D}}{ }_{\mathrm{t}}$.

$$
\begin{array}{r}
\overline{\operatorname{Ln}\left[P_{(t-1)}\right]}=-2.5165+2.1910 \cdot \operatorname{Ln}\left[G D P_{t}\right]+0.0202 \cdot I P_{t}-0.1799 \cdot \operatorname{Ln}\left[T_{t}\right]+ \\
\left.+0.1991 \cdot \operatorname{Ln}\left[P_{t}^{O I L}\right]-1.5881 \cdot \operatorname{Ln}_{[} U_{S D I}\right]+0.0717 \cdot \operatorname{Ln}\left[\operatorname{LIBOR}_{(t-6)}\right]
\end{array}
$$

The equations (5.30) and (5.32) match the underlying economic theories. The equation above can be written differently as follows: 


\section{Equation (5.33): The Model of the LME Copper Price:}

$$
P_{(t-1)}^{C U}=e^{(-2.5165)} \cdot G D P_{t}^{(2.1910)} \cdot e^{(0.0202) \cdot I P_{t}} \cdot T_{t}^{(-0.1799)} \cdot P_{\text {OIL }(t)}^{(0.191)} \cdot U S D I_{t}^{(-1.5881)} \cdot \operatorname{LIBOR}_{(t-6)}^{(0.0717)}
$$

Where,

$$
\mathrm{P}_{(\mathrm{t}-1)}^{\mathrm{CU}}=\text { Price of copper in }(\mathrm{t}-1)^{\mathrm{th}} \text { month } \quad \mathrm{e}=\text { exponential constant }
$$$$
\mathrm{GDP}_{\mathrm{t}}=\text { Gross domestic product in } \mathrm{t}^{\text {th }} \text { month } \quad \mathrm{IP}_{\mathrm{t}}=\text { Global IP growth in } \mathrm{t}^{\text {th }} \text { month }
$$$$
\mathrm{T}=\text { Time variable that implies technology } \quad \mathrm{P}_{\mathrm{OIL}(\mathrm{t})}=\text { Price of oil in } \mathrm{t}^{\text {th }} \text { month }
$$

$$
\mathrm{USDI}_{\mathrm{t}}=\mathrm{U} . \mathrm{S} \text {. dollar index in } \mathrm{t}_{\mathrm{th}} \text { month } \quad \text { LIBOR }_{(\mathrm{t}-6)}=\mathrm{LIBOR}-1 \mathrm{Y} \text { in }(\mathrm{t}-6)^{\mathrm{th}} \text { month }
$$

The meaning of the resulting coefficient estimates are (holding all else is being constant):

- If GDP in year (t) increases by $1 \%$ while the other Xs are held constant, then the price of copper in year (t-1) will increase by $2.19 \%$.

- If $\mathrm{T}$ in year (t) increases by $1 \%$ while the other Xs are held constant, then the price of copper in year (t-1) will decrease by $0.18 \%$.

- If $\mathrm{P}_{\mathrm{OIL}}$ in year $(\mathrm{t})$ increases by $1 \%$ while the other $\mathrm{Xs}$ are held constant, then the price of copper in year (t-1) will increase by $0.20 \%$.

- If USDI in year (t) increases by $1 \%$ while the other Xs are held constant, then the price of copper in year ( $\mathrm{t}-1)$ will decrease by $1.59 \%$.

- If 1-year LIBOR in year (t-6) increases by $1 \%$ while the other Xs are held constant, then the price of copper in year (t-1) will increase by $0.07 \%$.

- If Global IP growth in year ( $\mathrm{t}$ ) increases by 1 percentage point while the other Xs are held constant, then the price of copper in year (t-1) will increase by $2.02 \%(0.0202$ multiplied by 100). 
The GDP, IP and currency versus copper price links are much stronger than the other explanatory variables to price relationships, according to these results. In addition, the model results conclude that copper prices have a strong inverse relationship with the foreign exchange value of the dollar. 


\section{CHAPTER VI:}

\section{MONTE-CARLO SIMULATION ANALYSIS \\ FOR THE LME COPPER PRICES}

\subsection{SHORT TERM FORECASTING OF COPPER PRICES}

A forecast is a quantitative estimate about the likelihood of future values based on previous information. Forecasting methods can be classified as either time series model or causal models. Both models use time series data to predict future values. A causal model starts with a theory that identifies the explanatory variables that affect the dependent variable. After establishing a statistical relationship between the dependent and independent variables, a forecast is generated by substituting future values of the independent (causal) variables into the equation of the statistical relationship. A single equation model is a simple version of a causal model, but simultaneous models often are theoretically stronger. Causal models are econometric models and should be based on an econometric theory; therefore, the models can make "what if" predictions. Consequently, one of the principle purposes for constructing these econometric models is forecasting.

In contrast, a time series forecast is a different approach from a causal model because it is generated by using time as the underlying determinant. There are two reasons that causal models may not be accurate. At first, establishing a close statistical relationship between the dependent variable and explanatory variables is hard. Secondly, forecasts of future values of the explanatory variables are inaccurate even though a close relationship exists (Oyen, 1991, p. 392-393). Therefore, causal model application to commodity price 
forecasting has been limited. Selecting a forecasting technique depends on a tradeoff between accuracy and cost of forecasting. Finally, the outcome of forecasting is to help decision makers and improve today's decisions.

Forecasting can be divided into ex post and ex ante forecasts. The difference is that for ex post forecasting, the observations on both endogenous and exogenous variables are known with certainty because the forecast period is certain from the past until the present. In addition, an ex post forecast is checked against the existing or actual data and can be used to evaluate a forecasting model. By contrast, an ex ante forecast predicts values of the dependent variable using explanatory variables, which may or may not be known with certainty (Pindyck, Rubinfeld, 1981, p.204). The distinction between ex post and ex ante forecasting is shown in figure (6.1).

Figure (6.1): Ex Post and Ex Ante Forecasts

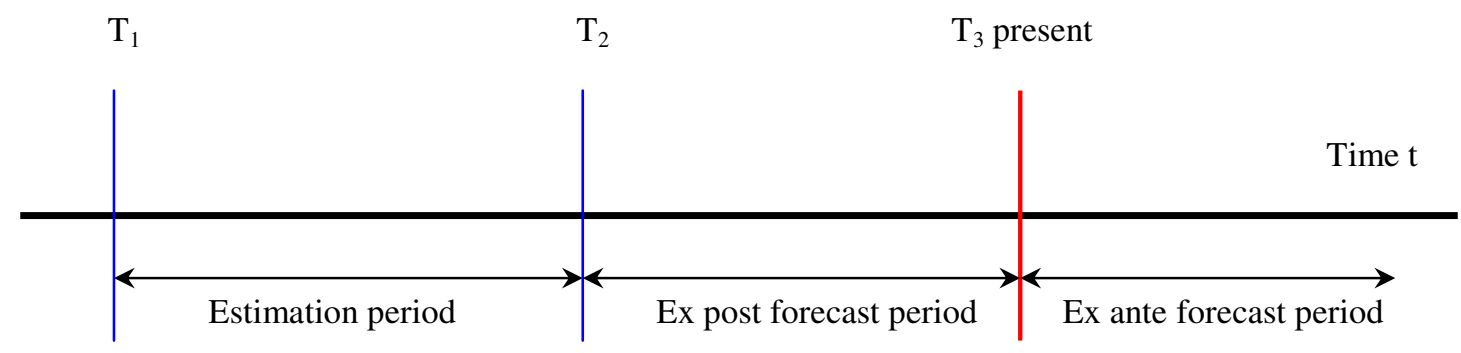

Actual and modeled prices of the LME copper are plotted in figure (6.2) using the price model (5.33), which was developed in the previous chapter. The observation period was between January 1995 and December 2004, so it is an ex-post forecast. 
Figure (6.2): Actual and Modeled Prices (Jan-95 to Dec-04)

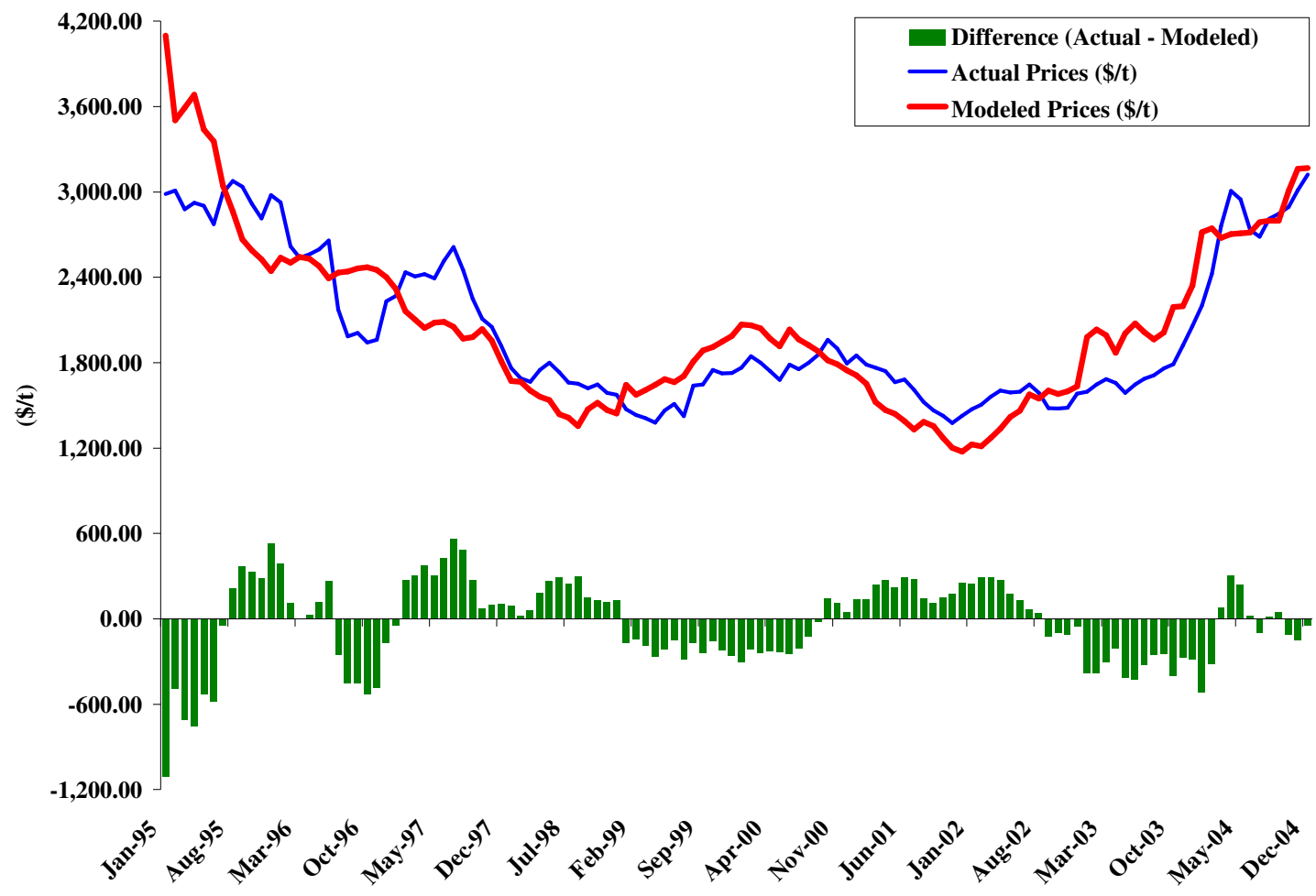

The outcome of the results of the model (5.33) is a forecast of monthly copper prices on the LME between January 2005 and December 2007. Forecasts for the copper price variable are obtained through solving the equation (5.30) of the supply and demand system. The projections of the exogenous variables including $\mathrm{GDP}_{t}, \mathrm{IP}_{\mathrm{t}}, \mathrm{T}_{\mathrm{t}}, \mathrm{P}_{\mathrm{OIL}(\mathrm{t})}, \mathrm{USDI}_{\mathrm{t}}$ and $\operatorname{LIBOR}(1 \mathrm{Y})_{(\mathrm{t}-6)}$ are necessary to be known in order to carry out copper price forecasts. These values are obtained from simulation results. By substituting these exogenous values into equation (5.33), monthly copper price forecasts for January 2005 December 2007 were obtained. However, not all forecasts of the exogenous variables were available and known. Therefore, a Monte-Carlo simulation is necessary to simulate these unknown exogenous variables and to obtain the forecasts of the endogenous variable of lagged copper price. 


\subsection{MONTE-CARLO SIMULATION ANALYSIS}

There are six exogenous variables including world GDP, global IP growth, time as a proxy for technological change, the price of oil, the U.S. dollar index, and the 6-month lagged LIBOR(1Y) in the copper price model, which is represented in equation (5.33).

$P_{(t-1)}^{C U}=e^{(-2.5165)} \cdot G D P_{t}^{(2.1910)} \cdot e^{(0.0202) \cdot I P_{t}} \cdot T_{t}^{(-0.1799)} \cdot P_{\text {OIL }(t)}^{(0.1991)} \cdot U_{S D I}^{(-1.5881)} \cdot \operatorname{LIBOR}_{t(-6)}^{(0.0717)}$

The time variable increases by one, starting from 120 in December 2004.

The following random elements were built in the copper price model:

- World GDP is expected to grow by $4.371 \%, 4.267 \%, 4.233 \%$ in 2005,2006 and 2007 respectively, according to the World Economic Outlook of the International Monetary Fund (The IMF, WEO March 2005, Figures: Global Indicator Growth Rates). Therefore, the yearly GDP is forecasted to be $\$ 36,567.099, \$ 38,127.417$, and $\$ 39,741.350$ billion in 2005,2006 , and 2007 respectively, using the following formula.

$$
\mathrm{GDP}_{\mathrm{t}}=\mathrm{GDP}_{(\mathrm{t}-1)} \cdot\left[1+\mathrm{E}(\mathrm{g})_{\mathrm{t}}\right]
$$

where, $\mathrm{GDP}_{\mathrm{t}}=$ world GDP in year $\mathrm{t} ; \quad \mathrm{E}(\mathrm{g})_{\mathrm{t}}=$ expected growth of GDP in year $\mathrm{t}$

Monthly GDP forecasts were calculated by dividing the annual GDP estimates into the number of months per year and these values were calculated as $\$ 3,047.258, \$ 3,177.285$, and $\$ 3,311.779$ billion in 2005, 2006 and 2007 respectively. The GDP variable is a normal distribution with a mean value of $\$ 3,047.258$ in each month of $2005, \$ 3,177.285$ in 2006 and $\$ 3,311.779$ in 2007, 
and a standard deviation of 121.21 in every month through $2005-2007$, which is defined on monthly data between 2002 and 2004 .

- IP growth: A normal distribution with a mean value of 6.22 and a standard deviation of 0.81 ;

- Price of oil: A normal distribution with a mean value of $\$ 41.44$ and a standard deviation of 5.85;

- U.S. dollar index: A normal distribution with a mean value of 85.41 and a standard deviation of 2.61;

- LIBOR (1Y): A normal distribution with a mean value of 1.5795 and a standard deviation of 0.36 .

The random numbers vary uniformly between 0 and 1 . Since GDP, IP growth, the price of oil, the U.S. dollar index and the 6-month lagged LIBOR(1Y) are normally distributed, MS Excel has a built in function that can be used (NORMINV). The standard deviations and the mean values in 2005-2007 were calculated based on the most recent year data, 2004 (i.e. between January 2004 and December 2004) for IP growth, the price of oil, the U.S. dollar index and the 6-month lagged LIBOR(1Y) variables.

At least 500 to 1000 simulation runs are required to estimate a single variable forecast in an econometric analysis; thus, there are 1000 simulations run (number of observations) in each monthly forecast of copper prices. For instance, a specific month forecast is calculated by averaging 1000 observation runs for this specific month.

The average of the forecasts, standard deviations and the coefficient of variations are shown in table below. 
Table 6.1: The Results of the Simulation

\begin{tabular}{|c|c|c|c|c|}
\hline Year & Month & $\begin{array}{c}\mathrm{Cu} \text { Price Forecasts } \\
(\$ / \mathrm{t})\end{array}$ & $\begin{array}{l}\text { Standard } \\
\text { Deviation }\end{array}$ & $\begin{array}{c}\text { Coefficient of } \\
\text { Variation }\end{array}$ \\
\hline \multirow{12}{*}{2005} & Jan. & $3,065.71$ & 320.91 & 0.11 \\
\hline & Feb. & $3,072.51$ & 325.38 & 0.11 \\
\hline & Mar. & $3,081.73$ & 327.06 & 0.11 \\
\hline & Apr. & $3,064.20$ & 323.81 & 0.11 \\
\hline & May & $3,048.64$ & 325.04 & 0.11 \\
\hline & Jun. & $3,039.66$ & 332.93 & 0.11 \\
\hline & Jul. & $3,066.99$ & 340.11 & 0.11 \\
\hline & Aug. & $3,042.47$ & 322.75 & 0.11 \\
\hline & Sep. & $3,046.96$ & 333.12 & 0.11 \\
\hline & Oct. & $3,037.93$ & 322.68 & 0.11 \\
\hline & Nov. & $3,026.96$ & 315.45 & 0.10 \\
\hline & Dec. & $3,310.11$ & 341.18 & 0.10 \\
\hline \multirow{12}{*}{2006} & Jan. & $3,337.28$ & 352.40 & 0.10 \\
\hline & Feb. & $3,351.26$ & 343.64 & 0.10 \\
\hline & Mar. & $3,292.40$ & 346.09 & 0.10 \\
\hline & Apr. & $3,305.33$ & 345.51 & 0.10 \\
\hline & May & $3,298.22$ & 337.88 & 0.10 \\
\hline & Jun. & $3,292.92$ & 343.84 & 0.10 \\
\hline & Jul. & $3,293.49$ & 338.33 & 0.10 \\
\hline & Aug. & $3,288.46$ & 337.06 & 0.10 \\
\hline & Sep. & $3,290.09$ & 336.61 & 0.10 \\
\hline & Oct. & $3,281.60$ & 325.38 & 0.10 \\
\hline & Nov. & $3,265.96$ & 329.58 & 0.10 \\
\hline & Dec. & $3,567.67$ & 355.93 & 0.10 \\
\hline \multirow{12}{*}{2007} & Jan. & $3,576.32$ & 360.45 & 0.10 \\
\hline & Feb. & $3,577.94$ & 365.49 & 0.10 \\
\hline & Mar. & $3,569.52$ & 365.09 & 0.10 \\
\hline & Apr. & $3,570.14$ & 358.49 & 0.10 \\
\hline & May & $3,556.49$ & 362.98 & 0.10 \\
\hline & Jun. & $3,569.26$ & 368.17 & 0.10 \\
\hline & Jul. & $3,532.42$ & 364.92 & 0.10 \\
\hline & Aug. & $3,532.79$ & 365.72 & 0.10 \\
\hline & Sep. & $3,540.91$ & 369.43 & 0.10 \\
\hline & Oct. & $3,541.22$ & 361.29 & 0.10 \\
\hline & Nov. & $3,521.37$ & 343.75 & 0.10 \\
\hline & Dec. & $3,528.84$ & 369.38 & 0.10 \\
\hline
\end{tabular}

Considering the probability of the copper price distribution range is better than using these average forecasts for a risk management decision. 
Table 6.2: Probability Distributions of Copper Price Forecasts

\begin{tabular}{|c|c|c|c|c|c|c|c|c|c|c|c|c|}
\hline \multirow[t]{2}{*}{ Months } & \multicolumn{12}{|c|}{ Probabilities for monthly average copper prices to be greater than specific strike prices } \\
\hline & $>2500$ & $>2600$ & $>2700$ & $>2800$ & $>2900$ & $>3000$ & $>3100$ & $>3200$ & $>3300$ & $>3400$ & $>3500$ & $>3600$ \\
\hline Jan-05 & 0.97 & 0.94 & 0.88 & 0.80 & 0.69 & 0.57 & 0.44 & 0.32 & 0.22 & 0.15 & 0.09 & 0.05 \\
\hline Feb-05 & 0.97 & 0.93 & 0.88 & 0.80 & 0.69 & 0.57 & 0.43 & 0.33 & 0.23 & 0.13 & 0.09 & 0.05 \\
\hline Mar-05 & 0.97 & 0.93 & 0.86 & 0.77 & 0.65 & 0.52 & 0.40 & 0.30 & 0.20 & 0.13 & 0.08 & 0.04 \\
\hline Apr-05 & 0.96 & 0.92 & 0.85 & 0.76 & 0.67 & 0.55 & 0.43 & 0.29 & 0.21 & 0.15 & 0.08 & 0.05 \\
\hline May-05 & 0.96 & 0.92 & 0.85 & 0.76 & 0.67 & 0.53 & 0.42 & 0.31 & 0.21 & 0.13 & 0.07 & 0.04 \\
\hline Jun-05 & 0.98 & 0.94 & 0.86 & 0.77 & 0.67 & 0.55 & 0.41 & 0.29 & 0.20 & 0.13 & 0.07 & 0.04 \\
\hline Jul-05 & 0.96 & 0.91 & 0.86 & 0.77 & 0.66 & 0.54 & 0.42 & 0.29 & 0.21 & 0.14 & 0.09 & 0.04 \\
\hline Aug-05 & 0.95 & 0.91 & 0.84 & 0.75 & 0.64 & 0.52 & 0.41 & 0.29 & 0.18 & 0.10 & 0.06 & 0.03 \\
\hline Sep-05 & 0.95 & 0.91 & 0.85 & 0.75 & 0.64 & 0.52 & 0.38 & 0.28 & 0.19 & 0.12 & 0.06 & 0.04 \\
\hline Oct-05 & 0.96 & 0.93 & 0.87 & 0.77 & 0.65 & 0.53 & 0.39 & 0.27 & 0.18 & 0.11 & 0.07 & 0.03 \\
\hline Nov-05 & 0.96 & 0.92 & 0.86 & 0.77 & 0.64 & 0.50 & 0.38 & 0.26 & 0.18 & 0.10 & 0.06 & 0.04 \\
\hline Dec-05 & 1.00 & 0.99 & 0.96 & 0.91 & 0.87 & 0.79 & 0.70 & 0.58 & 0.47 & 0.33 & 0.24 & 0.17 \\
\hline Jan-06 & 1.00 & 0.99 & 0.98 & 0.95 & 0.90 & 0.81 & 0.72 & 0.60 & 0.46 & 0.34 & 0.25 & 0.18 \\
\hline Feb-06 & 1.00 & 0.99 & 0.97 & 0.93 & 0.87 & 0.79 & 0.68 & 0.56 & 0.44 & 0.33 & 0.23 & 0.14 \\
\hline Mar-06 & 0.99 & 0.98 & 0.96 & 0.93 & 0.86 & 0.79 & 69 & .55 & .42 & 0.30 & 0.21 & 0.14 \\
\hline Apr-06 & 0.99 & 0.98 & 0.96 & 0.92 & 0.86 & 0.79 & 0.68 & 0.56 & 0.43 & 0.34 & 0.25 & 0.16 \\
\hline Мay-06 & 0.99 & 0.98 & 0.97 & 0.92 & 0.87 & 0.81 & 0.70 & 0.57 & 0.44 & 0.32 & 0.23 & 0.15 \\
\hline Jun-06 & 0.99 & 0.99 & 0.97 & 0.92 & 0.85 & 0.77 & 0.66 & 0.54 & 0.41 & 0.29 & 0.21 & 0.16 \\
\hline Jul-06 & 1.00 & 0.99 & 0.96 & 0.93 & 0.86 & 0.79 & 0.68 & 0.54 & 0.42 & 0.31 & 0.22 & 0.15 \\
\hline Aug-06 & 0.99 & 0.98 & 0.95 & 0.92 & 0.87 & 0.79 & 0.68 & 0.55 & 0.44 & 0.31 & 0.20 & 0.14 \\
\hline Sep-06 & 0.99 & 0.98 & 0.96 & 0.93 & 0.85 & 0.75 & 0.65 & 0.54 & 0.44 & 0.32 & 0.21 & 0.13 \\
\hline Oct-06 & 0.99 & 0.98 & 0.96 & 0.93 & 0.87 & 0.78 & 0.68 & 0.57 & 0.41 & 0.31 & 0.21 & 0.13 \\
\hline Nov-06 & 1.00 & 0.98 & 0.95 & 0.92 & 0.86 & 0.77 & 0.67 & 0.52 & 0.40 & 0.30 & 0.20 & 0.13 \\
\hline Dec-06 & 1.00 & 1.00 & 0.99 & 0.99 & 0.98 & 0.95 & 0.90 & 0.85 & 0.76 & 0.64 & 0.52 & 0.42 \\
\hline Jan-07 & 1.00 & 1.00 & 1.00 & 0.98 & 0.97 & 0.94 & 0.90 & 0.83 & 0.74 & 0.65 & 0.54 & 0.40 \\
\hline Feb-07 & 1.00 & 1.00 & 1.00 & 0.99 & 0.97 & 0.94 & 0.88 & 0.81 & 0.73 & 0.63 & 0.51 & 0.39 \\
\hline Mar-07 & 1.00 & 1.00 & 1.00 & 0.99 & 0.97 & 0.94 & 0.90 & 0.82 & 0.73 & 0.62 & 0.51 & 0.38 \\
\hline Apr-07 & 1.00 & 1.00 & 0.99 & 0.99 & 0.97 & 0.95 & 0.90 & 0.83 & 0.73 & 0.63 & 0.51 & 0.40 \\
\hline Мау-07 & 1.00 & 1.00 & 0.99 & 0.99 & 0.97 & 0.93 & 0.88 & 0.81 & 0.72 & 0.60 & 0.48 & 0.37 \\
\hline Jun-07 & 1.00 & 1.00 & 0.99 & 0.99 & 0.97 & 0.94 & 0.88 & 0.81 & 0.70 & 0.60 & 0.48 & 0.35 \\
\hline Jul-07 & 1.00 & 1.00 & 0.99 & 0.98 & 0.96 & 0.93 & 0.87 & 0.81 & 0.72 & 0.62 & 0.49 & 0.37 \\
\hline Aug-07 & 1.00 & 1.00 & 0.99 & 0.98 & 0.97 & 0.93 & 0.87 & 0.80 & 0.71 & 0.59 & 0.48 & 0.37 \\
\hline Sep-07 & 1.00 & 1.00 & 0.99 & 0.99 & 0.97 & 0.94 & 0.88 & 0.80 & 0.71 & 0.60 & 0.47 & 0.37 \\
\hline Oct-07 & 1.00 & 1.00 & 0.99 & 0.98 & 0.97 & 0.93 & 0.88 & 0.81 & 0.73 & 0.61 & 0.49 & 0.38 \\
\hline Nov-07 & 1.00 & 1.00 & 1.00 & 0.98 & 0.96 & 0.93 & 0.88 & 0.81 & 0.71 & 0.59 & 0.48 & 0.35 \\
\hline Dec-07 & 1.00 & 1.00 & 1.00 & 0.99 & 0.97 & 0.94 & 0.88 & 0.81 & 0.70 & 0.58 & 0.47 & 0.34 \\
\hline
\end{tabular}

As can be seen from these results, the monthly average price of copper on the LME will likely be greater than $\$ 3,000$ per tonne until 2007 . In 2006 , monthly average prices may be greater than $\$ 3,200$, but it is unlikely to be greater than $\$ 3,300$ because the probability distributions of being greater than $\$ 3,300$ were centered around 0.5 .

In 2007 , monthly average prices could be around $\$ 3,300-\$ 3,400$ per tonne. 


\section{CHAPTER VII:}

\section{EVALUATION OF RISK}

\section{MANAGEMENT STRATEGIES}

\subsection{OPTIONS STRATEGIES}

In this part, options strategies and spreads will be considered for copper as the underlying asset. Options can be used to create a wide range of different payoff functions depending on the situations of upturns or downturns in the price of the underlying asset (Hull, 2002, p. 202).

As we know from the data analysis in chapter 3, options quotes were chosen in two consecutive days on 21 February and 22 February 2005. The second day's data were used to define and suggest a strategy, which combines positions in the underlying asset and options. In other words, evaluations of risk management strategies in this chapter are based on the information and indications from 22 February 2005 because both call and put options' quotes with different maturities and strike prices were obtained on this date. The pricing indications are only good at the moment when they are priced. This is referred as a disclaimer and all metal dealers use similar disclaimers on indications. Each time period market price, variables and indications change and this means that a dealer cannot guarantee the same level of indications, so customers must call dealers on the telephone for a "live price". Therefore, the date of data obtained for 22 February 2005 was chosen as the value date in this chapter. 
The time value of money has not been ignored when calculating the payoff from a trading and risk management strategy; thus, the profit is calculated as a final payoff, plus the future value of initial payoff of a strategy. Equation (7.1) demonstrates the calculation of total payoff of a trading and risk management strategy.

$$
\text { Payoff }_{\text {TотаL }}=\text { Payoff }_{0} \cdot e^{r \cdot(T / 365)}+\text { Payoff }_{T}
$$

where, Payoff $\mathrm{TOTAL}_{\mathrm{T}}=$ the total payoff of a strategy at time $\mathrm{T}$ e $=$ exponential constant

$$
\begin{array}{ll}
\text { Payoff }_{(0)}=\text { the payoff at time zero } & \mathrm{T}=\text { the time to maturity (days) } \\
\text { Payoff }_{(\mathrm{T})}=\text { the payoff at time } \mathrm{T} & \mathrm{r}=\text { the risk free interest rate }(\%)
\end{array}
$$

The following table gives the numerical values of essential variables including the risk free interest rate (percent per year) and time to maturity (by days) in order to determine the future value of initial payoffs of trading and risk management strategies.

The risk free interest rate is defined by formula (3.10) in chapter 3 based on T-bill discount rate at constant maturities that is closest to an option's expiration date.

\begin{tabular}{|c|c|c|c|c|c|c|c|c|c|c|c|c|}
\hline \multirow{2}{*}{$\begin{array}{l}\text { The Date of Data Released } \\
22-\mathrm{Feb}-05\end{array}$} & \multicolumn{12}{|c|}{ Options } \\
\hline & Jan-06 & Feb-06 & Mar-06 & Apr-06 & May-06 & Jun-06 & Jul-06 & Aug-06 & Sep-06 & Oct-06 & Nov-06 & Dec-06 \\
\hline $\begin{array}{l}\text { The Risk Free } \\
\text { Interest Rate }(\%)\{r\}\end{array}$ & 3.21 & 3.21 & 3.21 & 3.21 & 3.21 & 3.21 & 3.21 & 3.64 & 3.64 & 3.64 & 3.64 & 3.64 \\
\hline $\begin{array}{l}\text { The Time to } \\
\text { Maturity (day }\end{array}$ & 345 & 373 & 406 & 435 & 465 & 497 & 526 & 559 & 588 & 618 & 650 & 680 \\
\hline \multirow{2}{*}{$\begin{array}{l}\text { The Date of Data Released } \\
\text { 22-Feb-05 }\end{array}$} & \multicolumn{12}{|c|}{ Options } \\
\hline & Jan-07 & Feb-07 & Mar-07 & Apr-07 & May-07 & Jun-07 & Jul-07 & Aug-07 & Sep-07 & Oct-07 & Nov-07 & $\begin{array}{l}\text { Dec-07 } \\
\end{array}$ \\
\hline $\begin{array}{l}\text { The Risk Free } \\
\text { Interest Rate }(\%)\{r\}\end{array}$ & 3.21 & 3.21 & 3.21 & 3.21 & 3.21 & 3.21 & 3.21 & 3.89 & 3.89 & 3.89 & 3.89 & 3.89 \\
\hline $\begin{array}{l}\text { The Time to } \\
\text { Maturity (days) }\{\mathrm{T}\}\end{array}$ & 710 & 738 & 770 & 799 & 832 & 861 & 891 & 924 & 952 & 983 & 1015 & 1045 \\
\hline
\end{tabular}

\section{Table 7.1: The Risk Free Interest Rates and Time to Maturities for Selected Options}

\begin{tabular}{|l|c|c|c|c|c|c|}
\hline The Date of Data Released & \multicolumn{7}{|c|}{ Options } \\
\cline { 2 - 7 } 22-Feb-05 & Jul-05 & Aug-05 & Sep-05 & Oct-05 & Nov-05 & Dec-05 \\
\hline $\begin{array}{l}\text { The Risk Free } \\
\text { Interest Rate (\%) }\{\mathrm{r}\}\end{array}$ & 3.00 & 3.00 & 3.00 & 3.00 & 3.21 & 3.21 \\
\hline $\begin{array}{l}\text { The Time to } \\
\text { Maturity (days) }\{\mathrm{T}\}\end{array}$ & 161 & 192 & 224 & 253 & 283 & 316 \\
\hline
\end{tabular}


A spread trading strategy involves trading a position in two or more options of the same type: either calls or puts. Based upon the analysis in the previous chapter and the data obtained for 22 February 2005, the opportunities made available by options strategies will be researched here. First, let us start with spreads.

\subsubsection{BULL SPREADS:}

A bull spread is used by investors, option traders and commodity consumers when the underlying asset price is expected to increase. Bull spreads can be created by taking a long position on a call option with a specific strike price and a short position on a call option with a higher strike price. Both options have the same maturity date.

\section{* Bull Spreads Using Call Options:}

Table (7.6) demonstrates all possible bull spread strategies using call options for 2006 and 2007 based on the indications of 22 February 2005. There are 5 different spreads each month, so there are a total of 120 bull spread strategies developed using call options for 24 months between Jan-06 and Dec-07.

Figure (7.1) shows the probabilities for copper prices to be greater than specific strike prices resulting from the analysis in chapter 6 . The best opportunity for using a bull spread with call options in our example case starts from January 2006 until December 2007 using options with strike prices $\$ 3,100$ and $\$ 3,200$ if the probability of $60 \%$ or greater is accepted. From table (6.2) in chapter 6, monthly copper prices will likely be around $\$ 3,300-\$ 3,400$ starting from December 2006 because the probabilities of copper price being more than 3300-3400 are greater than 60-70\% after that date for strike prices of 3300 and 3400 only. 
Figure (7.1): Probabilities for Copper Prices to be Greater than Specific Strike Prices

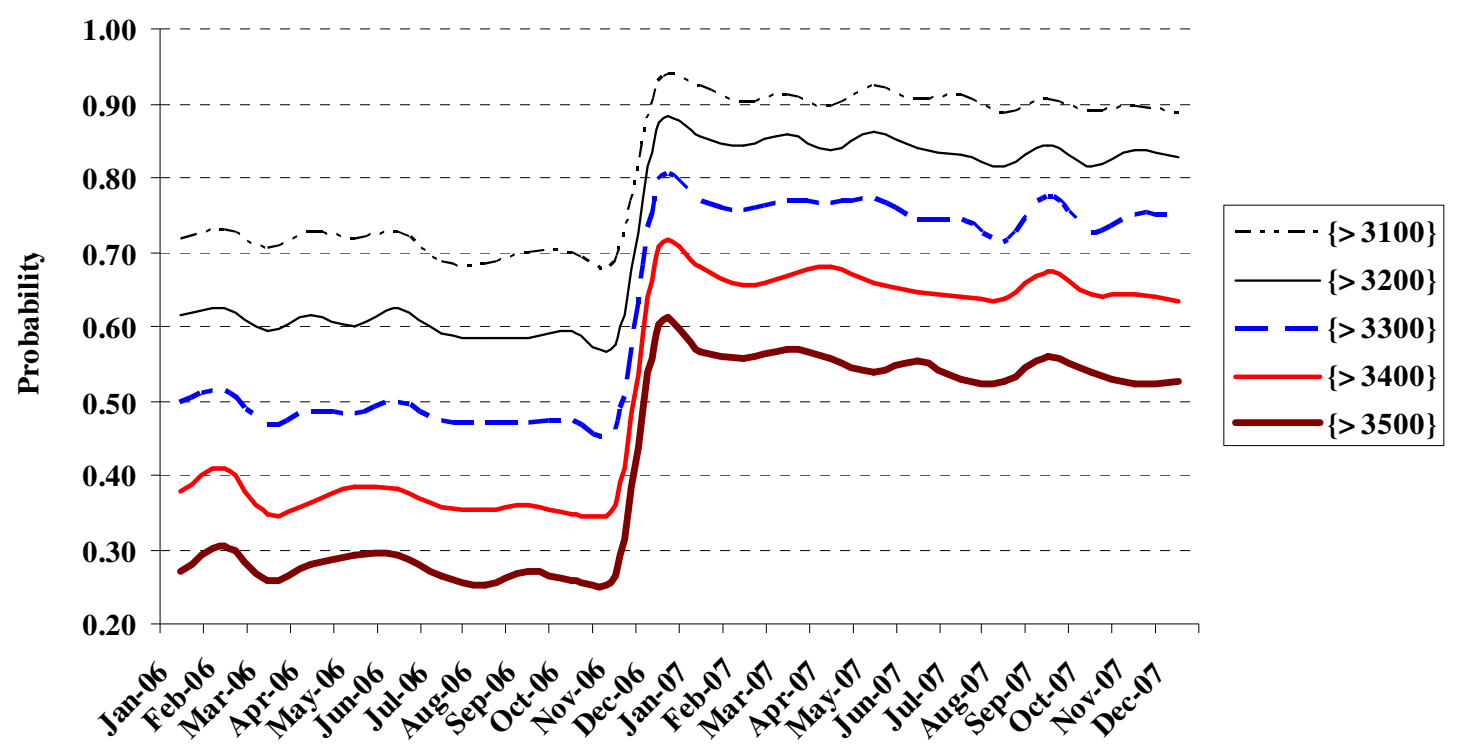

Therefore, based on the analysis in chapter 6, the best bull spread strategies involving call options would be in the months between Jan-06 and Dec-07:

a) In 2006:

- Long position in a call option at $\$ 3,100 / \mathrm{t}$ and short position in a call option at $\$ 3,200 /$ for January 2006 through December 2007.

12 bull spread strategies using call options would be potentially profitable.

b) In 2007:

- Long position in a call option at $\$ 3,100 / t$ and short position in a call option at $\$ 3,200 /$ for January 2007 through December 2007;

- Long position in a call option at $\$ 3,200 / t$ and short position in a call option at $\$ 3,300 /$ for January 2007 through December 2007;

- Long position in a call option at $\$ 3,300 / t$ and short position in a call option at $\$ 3,400 /$ for January 2007 through December 2007.

36 bull spread strategies using call options would be potentially profitable. 
As a result, there are in total 48 bull spread strategies using call options that would be potentially profitable and useful in 2006 and 2007. From these 48 strategies, let us construct the best four bull spreads, which have highest positive total payoffs (gain) and lowest negative total payoffs (loss).

\section{Bull Spread for January 2006}

- Spot Price (22 Feb-05):

- Asian Call Options:

- Positions:
$\$ 3,367$

January-2006

Long Call @ 3100: $\quad$ premium $\$ 159.99$

Short Call @ 3200: $\quad$ premium $\$ 133.05$

Table 7.2: Profit from a Bull Spread (Jan-06 Call options, X: 3100/3200)

\begin{tabular}{cccc}
\hline $\begin{array}{c}\text { Copper price } \\
\text { range }\end{array}$ & $\begin{array}{c}\text { Profit from long } \\
\text { call @ 3100 }\end{array}$ & $\begin{array}{c}\text { Profit from short } \\
\text { call @ 3200 }\end{array}$ & $\begin{array}{c}\text { Total } \\
\text { profit }\end{array}$ \\
\hline $\mathrm{S}_{\mathrm{AVG}}>3200$ & $\mathrm{~S}_{\mathrm{AVG}}-3264.92$ & $3337.15-\mathrm{S}_{\mathrm{AVG}}$ & 72.23 \\
$3200 \geq \mathrm{S}_{\mathrm{AVG}}>3100$ & $\mathrm{~S}_{\mathrm{AVG}}-3264.92$ & 137.15 & $\mathrm{~S}_{\mathrm{AVG}}-3127.77$ \\
$\mathrm{~S}_{\mathrm{AVG}} \leq 3100$ & -164.92 & 137.15 & -27.77 \\
\hline
\end{tabular}

If the average price of copper in January 2006 is greater than $\$ 3,200 /$ t the call option that was bought at $\$ 3,100$ would be exercised, so the payoff at the maturity of this long option position would equal the future value of initial payoff (premium) -\$164.92 plus $\left\{\mathrm{S}_{\mathrm{AVG}}-\$ 3,100\right\}$. Thus, the profit from long position in a call option at $\$ 3,100$ would be $\left\{\mathrm{S}_{\mathrm{AVG}}-\$ 3,264.92\right\}$. The call option that was written at $\$ 3,200$ would also be exercised if the average price of copper is greater than $\$ 3,200$. In this case, the payoff at the option's maturity of this short option position would be the future value of initial payoff (premium) $\$ 137.15$ minus $\left\{\mathrm{S}_{\mathrm{AVG}}-\$ 3,200\right\}$, so the profit from short position in a call option at $\$ 3200$ would be $\left\{\$ 3,337.15-S_{\mathrm{AVG}}\right\}$. Hence, the total payoff of the strategy would be $\$ 72.23$ if the average price of copper in January 2006 is greater than $\$ 3,200$.

If the average price of copper is between $\$ 3,200 / t$ and $\$ 3,100 / t$, the payoff from the option that was bought at $\$ 3,100$ would be $\left\{S_{\mathrm{AVG}}-\$ 3,264.92\right\}$ at the option's maturity. 
However, the call option that was written at $\$ 3,200$ would not be exercised, so the payoff at the option's maturity from this short option position would equal the future value of initial payoff $\$ 137.15$. The total payoff of the strategy would be $\left\{S_{A V G}-\$ 3,127.77\right\}$.

If the average price of copper is less than $\$ 3,100$, both options that were bought and written would not be exercised; therefore, the total payoff of the strategy would equal the sum of the future values of options' initial payoffs. In this case, a long call position at $\$ 3,100$ would result loss of $\$ 164.92$, and a short call position at $\$ 3,200$ would result profit of 137.15 , so the total payoff would result loss of $\$ 27.77$. Profit is made when $\left[\mathrm{S}_{\mathrm{AVG}(\mathrm{Jan}-2006)}>\$ 3,127.77\right]$ and is maximum of $\$ 72.23$ when $\left[\mathrm{S}_{\mathrm{AVG}(\mathrm{Jan}-2006)}>\$ 3,200\right]$.

Bull Spread for January 2007

- Spot Price (22 Feb-05):

- Asian Call Options:

- Positions:
$\$ 3,367$

January-2007

Long Call @ 3100: $\quad$ premium $\$ 122.86$

Short Call @ 3200: $\quad$ premium $\$ 103.13$

Table 7.3: Profit from a Bull Spread (Jan-07 Call options, X: 3100/3200)

\begin{tabular}{cccc}
\hline $\begin{array}{c}\text { Copper price } \\
\text { range }\end{array}$ & $\begin{array}{c}\text { Profit from long } \\
\text { call @ 3100 }\end{array}$ & $\begin{array}{c}\text { Profit from short } \\
\text { call @ 3200 }\end{array}$ & $\begin{array}{c}\text { Total } \\
\text { profit }\end{array}$ \\
\hline $\mathrm{S}_{\mathrm{AVG}}>3200$ & $\mathrm{~S}_{\mathrm{AVG}}-3231.87$ & $3310.70-\mathrm{S}_{\mathrm{AVG}}$ & 78.83 \\
$3200 \geq \mathrm{S}_{\mathrm{AVG}}>3100$ & $\mathrm{~S}_{\mathrm{AVG}}-3231.87$ & 110.70 & $\mathrm{~S}_{\mathrm{AVG}}-3121.17$ \\
$\mathrm{~S}_{\mathrm{AVG}} \leq 3100$ & -131.87 & 110.70 & -21.17 \\
\hline
\end{tabular}

Profit is made when $\left[\mathrm{S}_{\mathrm{AVG}(\mathrm{Jan}-2007)}>\$ 3,121.17\right]$ and is maximum of $\$ 78.83$ when $\left[\mathrm{S}_{\mathrm{AVG}(\mathrm{Jan}-2007)}>\$ 3,200\right]$.

\section{Bull Spread for January 2007}

- Spot Price (22 Feb-05):

- Asian Call Options:

- Positions:
$\$ 3,367$

January-2007

Long Call @ 3200: $\quad$ premium $\$ 103.13$

Short Call @ 3300: $\quad$ premium $\$ 86.30$ 
Table 7.4: Profit from a Bull Spread (Jan-07 Call options, X: 3200/3300)

\begin{tabular}{cccc}
\hline $\begin{array}{c}\text { Copper price } \\
\text { range }\end{array}$ & $\begin{array}{c}\text { Profit from long } \\
\text { call @ 3200 }\end{array}$ & $\begin{array}{c}\text { Profit from short } \\
\text { call @ 3300 }\end{array}$ & $\begin{array}{c}\text { Total } \\
\text { profit }\end{array}$ \\
\hline $\mathrm{S}_{\mathrm{AVG}}>3300$ & $\mathrm{~S}_{\mathrm{AVG}}-3310.70$ & $3392.63-\mathrm{S}_{\mathrm{AVG}}$ & 81.93 \\
$3300 \geq \mathrm{S}_{\mathrm{AVG}}>3200$ & $\mathrm{~S}_{\mathrm{AVG}}-3310.70$ & 92.63 & $\mathrm{~S}_{\mathrm{AVG}}-3218.07$ \\
$\mathrm{~S}_{\mathrm{AVG}} \leq 3200$ & -110.70 & 92.63 & -18.07 \\
\hline
\end{tabular}

Profit is made when $\left[\mathrm{S}_{\mathrm{AVG}(\mathrm{Jan}-2007)}>\$ 3,218.07\right]$ and is maximum of $\$ 81.93$ when

$\left[\mathrm{S}_{\mathrm{AVG}(\mathrm{Jan}-2007)}>\$ 3,300\right]$.

\section{Bull Spread for January 2007}

- Spot Price (22 Feb-05):

- Asian Call Options:

- Positions:
$\$ 3,367$

January-2007

Long Call @ 3300: $\quad$ premium $\$ 86.30$

Short Call @ 3400: $\quad$ premium $\$ 72.00$

Table 7.5: Profit from a Bull Spread (Jan-07 Call options, X: 3300/3400)

\begin{tabular}{cccc}
\hline $\begin{array}{c}\text { Copper price } \\
\text { range }\end{array}$ & $\begin{array}{c}\text { Profit from long } \\
\text { call @ 3300 }\end{array}$ & $\begin{array}{c}\text { Profit from short } \\
\text { call @ 3400 }\end{array}$ & $\begin{array}{c}\text { Total } \\
\text { profit }\end{array}$ \\
\hline $\mathrm{S}_{\mathrm{AVG}}>3400$ & $\mathrm{~S}_{\mathrm{AVG}}-3392.63$ & $3477.28-\mathrm{S}_{\mathrm{AVG}}$ & 84.65 \\
$3400 \geq \mathrm{S}_{\mathrm{AVG}}>3300$ & $\mathrm{~S}_{\mathrm{AVG}}-3392.63$ & 77.28 & $\mathrm{~S}_{\mathrm{AVG}}-3315.35$ \\
$\mathrm{~S}_{\mathrm{AVG}} \leq 3300$ & -92.63 & 77.28 & -15.35 \\
\hline
\end{tabular}

Profit is made when $\left[\mathrm{S}_{\mathrm{AVG}(\mathrm{Jan}-2007)}>\$ 3,315.35\right]$ and is maximum of $\$ 84.65$ when

$\mathrm{S}_{\mathrm{AVG}(\mathrm{Jan}-2007)}>\$ 3,400$.

Figure (7.2): Bull Spread Using Call Options Jan-06, X: 3100/3200

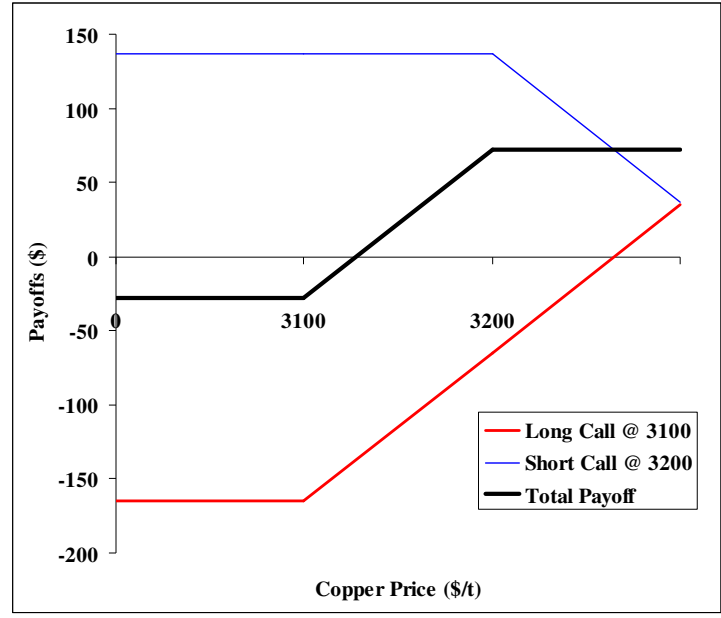

Figure (7.3): Bull Spread Using Call Options Jan-07, X: 3100/3200

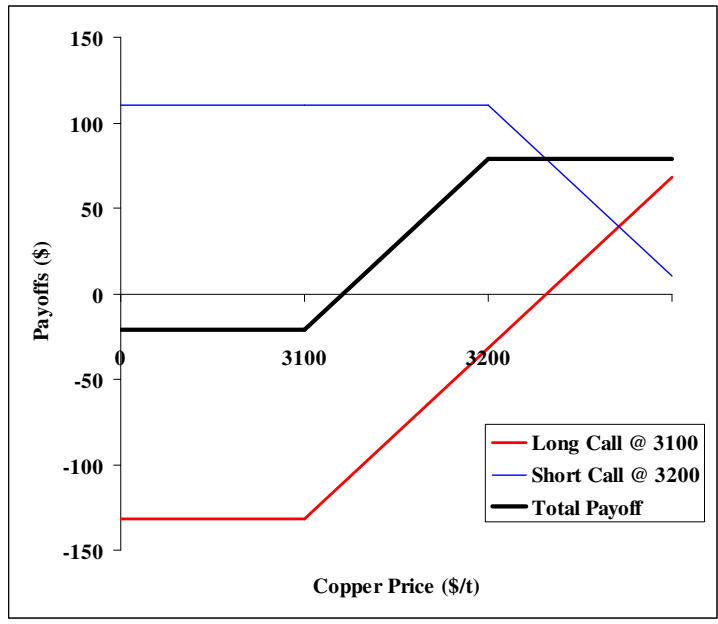


Figure (7.4): Bull Spread Using Call Options Jan-07, X: 3200/3300

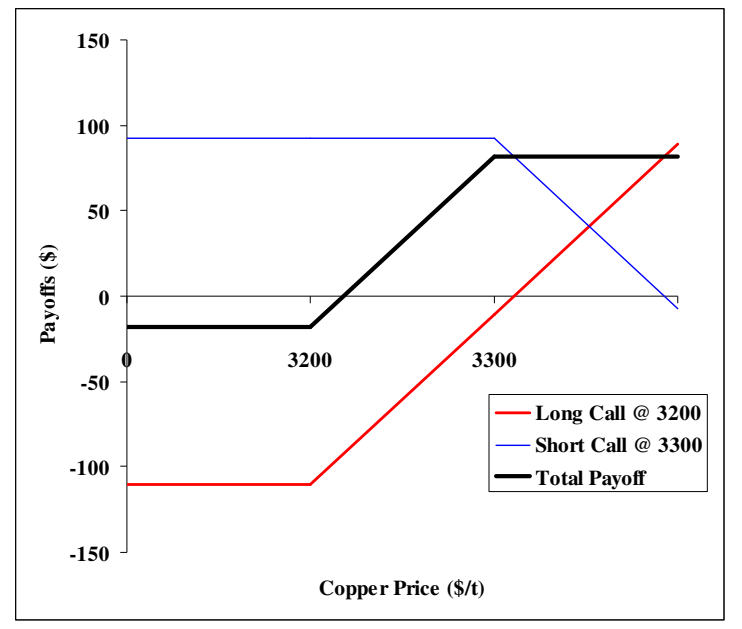

Figure (7.5): Bull Spread Using Call Options Jan-07, X: 3300/3400

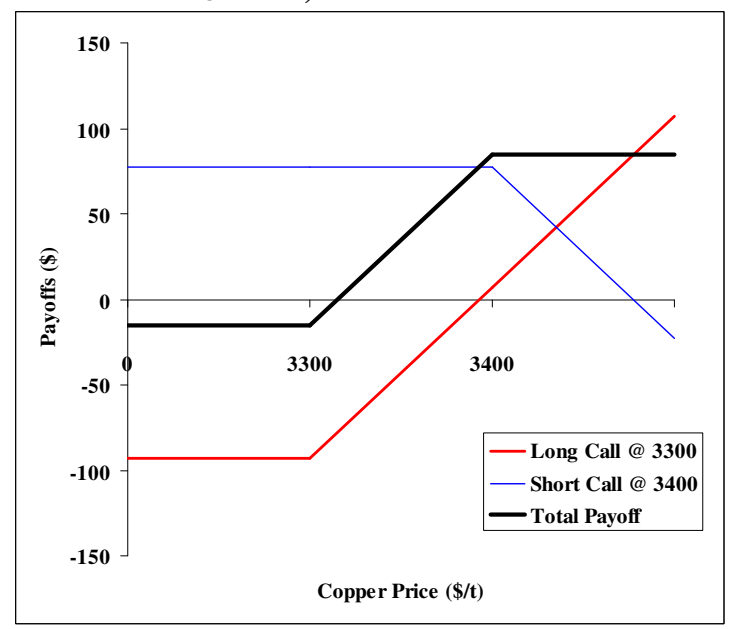

From these four best bull spread strategies, the spread for Jan-07 using call options at the strike prices $\$ 3,300 / \$ 3,400$ is better than for Jan-06 call options at the strike prices $\$ 3,100 / \$ 3,200$, for Jan-07 call options at the strike prices $\$ 3,100 / \$ 3,200$, and $\$ 3,200 / \$ 3,300$ because its restricted loss is lower and the gain is higher. If the monthly average copper price in January-07 is greater than $\$ 3,400$, an investor will gain $\$ 84.65 /$ t. If the price is between $\$ 3,300$ and $\$ 3,400$, the written call option will not be exercised, so the total payoff will be $\left\{S_{A V G}-\$ 3,315.35\right\}$. If the price falls below $\$ 3,300$, the investor's loss is restricted by the future value of initial investment of $\$ 15.35 / \mathrm{t}$ (the difference between the call options' prices). A call option's price always declines as the strike price increases, so the value of the option bought is always greater than the value of the option sold (Note: $\$ 86.30>\$ 72.00$ ). In conclusion, bull spread strategies using call options limit the investor's upside as well as the downside price risk. 
Table 7.6: The Payoffs of Bull Spreads Using Call Options for 2006 and 2007

\begin{tabular}{|c|c|c|c|c|c|c|c|c|c|c|c|c|c|c|c|c|c|c|c|}
\hline \multicolumn{4}{|c|}{ Call: Jan-06 } & \multicolumn{4}{|c|}{ Call: Jan-06 } & \multicolumn{4}{|c|}{ Call: Jan-06 } & \multicolumn{4}{|c|}{ Call: Jan-06 } & \multicolumn{4}{|c|}{ Call: Jan-06 } \\
\hline \multirow[b]{2}{*}{$\mathrm{S}$} & \multicolumn{3}{|c|}{ Payoff } & \multirow[b]{2}{*}{ S } & \multicolumn{3}{|c|}{ Payoff } & \multirow[b]{2}{*}{$\mathrm{S}$} & \multicolumn{3}{|c|}{ Payoff } & & & Payoff & & & & Payoff & \\
\hline & \begin{tabular}{|l|} 
Long \\
3100 \\
\end{tabular} & $\begin{array}{l}\text { Short } \\
3200\end{array}$ & Total & & $\begin{array}{l}\text { Long } \\
3200\end{array}$ & $\begin{array}{l}\text { Short } \\
3300\end{array}$ & Total & & $\begin{array}{l}\text { Long } \\
\mathbf{3 3 0 0}\end{array}$ & $\begin{array}{l}\text { Short } \\
3400\end{array}$ & Total & S & $\begin{array}{l}\text { Long } \\
3400\end{array}$ & $\begin{array}{l}\text { Short } \\
3500\end{array}$ & \begin{tabular}{|l|} 
Total \\
\end{tabular} & S & $\begin{array}{l}\text { Long } \\
3500\end{array}$ & $\begin{array}{l}\text { Short } \\
3600\end{array}$ & Total \\
\hline 0 & \begin{tabular}{|l|}
-164.92 \\
\end{tabular} & 137.15 & -27.77 & 0 & -137.15 & 113.48 & -23.67 & 0 & -113.48 & 93.46 & -20.02 & $\overline{0}$ & $\begin{array}{l}-93.46 \\
\end{array}$ & 76.64 & \begin{tabular}{|l|}
-16.82 \\
\end{tabular} & 0 & $\begin{array}{l}-76.64 \\
\end{array}$ & 62.59 & -14.05 \\
\hline 3100 & -164.92 & 137.15 & -27.77 & 3200 & -137.15 & 113.48 & -23.67 & 3300 & -113.48 & 93.46 & -20.02 & 3400 & -93.46 & 76.64 & -16.82 & 3500 & -76.64 & 62.59 & -14.05 \\
\hline 3200 & -64.92 & 137.15 & 72.23 & 3300 & -37.15 & 113.48 & 76.33 & 3400 & -13.48 & 93.46 & 79.98 & 3500 & 6.54 & 76.64 & 83.18 & 3600 & 23.36 & 62.59 & 85.95 \\
\hline 3300 & 35.08 & 37.15 & 72.23 & 3400 & 62.85 & 13.48 & 76.33 & 3500 & 86.52 & -6.54 & 79.98 & 3600 & 106.54 & -23.36 & 83.18 & 3700 & 123.36 & -37.41 & 85.95 \\
\hline Call: $\mathbf{F e}$ & b-06 & & & Call: $\mathbf{F e}$ & b-06 & & & Call: $\mathbf{F}$ & $0-06$ & & & Call: $\mathbf{F e}$ & -06 & & & Call: $\mathrm{Fe}$ & 06 & & \\
\hline $\mathrm{S}$ & & Payoff & & $\mathrm{S}$ & & Payoff & & $\mathrm{S}$ & & Payoff & & $\mathrm{S}$ & & Payoff & & $\mathrm{S}$ & & Payoff & \\
\hline & \begin{tabular}{|l|} 
Long \\
3100
\end{tabular} & $\begin{array}{l}\text { Short } \\
3200\end{array}$ & \begin{tabular}{|l|} 
Total \\
\end{tabular} & & $\begin{array}{l}\text { Long } \\
3200\end{array}$ & $\begin{array}{l}\text { Short } \\
3300\end{array}$ & Total & & $\begin{array}{l}\text { Long } \\
3300\end{array}$ & $\begin{array}{l}\text { Short } \\
3400\end{array}$ & Total & & $\begin{array}{l}\text { Long } \\
3400\end{array}$ & $\begin{array}{l}\text { Short } \\
3500\end{array}$ & \begin{tabular}{|l|} 
Total \\
\end{tabular} & & $\begin{array}{l}\text { Long } \\
3500\end{array}$ & $\begin{array}{l}\text { Short } \\
3600\end{array}$ & \begin{tabular}{|l|} 
Total \\
\end{tabular} \\
\hline 0 & -165.33 & $\begin{array}{l}137.49 \\
\end{array}$ & -27.84 & 0 & -137.49 & 113.76 & -23.73 & 0 & -113.76 & 93.69 & -20.07 & 0 & $\begin{array}{l}-93.69 \\
\end{array}$ & 76.83 & \begin{tabular}{|l|}
-16.86 \\
\end{tabular} & 0 & \begin{tabular}{l|}
-76.83 \\
\end{tabular} & 62.74 & -14.08 \\
\hline 3100 & -165.33 & 137.49 & -27.84 & 3200 & -137.49 & 113.76 & -23.73 & 3300 & -113.76 & 93.69 & -20.07 & 3400 & -93.69 & 76.83 & -16.86 & 3500 & -76.83 & 62.74 & -14.08 \\
\hline 3200 & -65.33 & 137.49 & 72.16 & 3300 & -37.49 & 113.76 & 76.27 & 3400 & -13.76 & 93.69 & 79.93 & 3500 & 6.31 & 76.83 & 83.14 & 3600 & 23.17 & 62.74 & 85.92 \\
\hline 3300 & 34.67 & 37.49 & 72.16 & 3400 & 62.51 & 13.76 & 76.27 & 3500 & 86.24 & -6.31 & 79.93 & 3600 & 106.31 & -23.17 & 83.14 & 3700 & 123.17 & -37.26 & 85.92 \\
\hline Call: $M$ & $a r-06$ & & & Call: $M$ & $\overline{\text { ar-06 }}$ & & & Call: $\mathbf{M}$ & $\mathrm{r}-06$ & & & Call: $M_{i}$ & $\overline{r-06}$ & & & Call: $\mathbf{M}$ & $r-06$ & & \\
\hline $\mathrm{s}$ & & Payoff & & $\mathrm{s}$ & & Payoff & & $\mathrm{s}$ & & Payoff & & $\mathrm{S}$ & & Payoff & & $\mathrm{S}$ & & Payoff & \\
\hline & $\begin{array}{l}\text { Long } \\
3100\end{array}$ & $\begin{array}{l}\text { Short } \\
\mathbf{3 2 0 0}\end{array}$ & Total & & $\begin{array}{l}\text { Long } \\
3200\end{array}$ & $\begin{array}{l}\text { Short } \\
\mathbf{3 3 0 0}\end{array}$ & Total & & $\begin{array}{l}\text { Long } \\
\mathbf{3 3 0 0}\end{array}$ & $\begin{array}{l}\text { Short } \\
3400\end{array}$ & Total & & $\begin{array}{l}\text { Long } \\
3400\end{array}$ & $\begin{array}{l}\text { Short } \\
3500\end{array}$ & \begin{tabular}{|l|} 
Total \\
\end{tabular} & & $\begin{array}{l}\text { Long } \\
3500\end{array}$ & $\begin{array}{l}\text { Short } \\
3600\end{array}$ & Total \\
\hline 0 & -165.81 & $\begin{array}{l}137.89 \\
\end{array}$ & -27.92 & 0 & -137.89 & 114.09 & -23.79 & 0 & -114.09 & 93.97 & -20.13 & 0 & \begin{tabular}{|c|}
-93.97 \\
\end{tabular} & 77.05 & \begin{tabular}{|l|}
-16.91 \\
\end{tabular} & 0 & \begin{tabular}{|c|}
-77.05 \\
\end{tabular} & \begin{tabular}{|l|}
62.93 \\
\end{tabular} & -14.13 \\
\hline 3100 & -165.81 & 137.89 & -27.92 & 3200 & -137.89 & 114.09 & -23.79 & 3300 & -114.09 & 93.97 & -20.13 & 3400 & -93.97 & 77.05 & -16.91 & 3500 & -77.05 & 62.93 & -14.13 \\
\hline 3200 & -65.81 & 137.89 & 72.08 & 3300 & -37.89 & 114.09 & 76.21 & 3400 & -14.09 & 93.97 & 79.87 & 3500 & 6.03 & 77.05 & 83.09 & 3600 & 22.95 & 62.93 & 85.87 \\
\hline 3300 & 34.19 & 37.89 & 72.08 & 3400 & 62.11 & 14.09 & 76.21 & 3500 & 85.91 & -6.03 & 79.87 & 3600 & 106.03 & -22.95 & 83.09 & 3700 & 122.95 & -37.07 & 85.87 \\
\hline Call: $A_{1}$ & $\mathrm{r}-06$ & & & Call: $A_{1}$ & pr-06 & & & Call: $\mathbf{A}$ & $r-06$ & & & Call: Ap & 806 & & & $\overline{\text { Call: } A}$ & $8-06$ & & \\
\hline$S$ & & Payoff & & S & & Payoff & & S & & Payoff & & $\mathrm{S}$ & & Payoff & & $S$ & & Payoff & \\
\hline & \begin{tabular}{|l|} 
Long \\
3100
\end{tabular} & $\begin{array}{l}\text { Short } \\
3200\end{array}$ & Total & & $\begin{array}{l}\text { Long } \\
3200\end{array}$ & $\begin{array}{l}\text { Short } \\
\mathbf{3 3 0 0}\end{array}$ & Total & & $\begin{array}{l}\text { Long } \\
3300\end{array}$ & $\begin{array}{l}\text { Short } \\
3400\end{array}$ & Total & & $\begin{array}{l}\text { Long } \\
3400\end{array}$ & $\begin{array}{l}\text { Short } \\
3500\end{array}$ & \begin{tabular}{|l|} 
Total \\
\end{tabular} & & $\begin{array}{l}\text { Long } \\
3500\end{array}$ & $\begin{array}{l}\text { Short } \\
3600\end{array}$ & Total \\
\hline 0 & -166.23 & \begin{tabular}{|c|}
138.24 \\
\end{tabular} & -27.99 & 0 & -138.24 & \begin{tabular}{ll|}
114.38 \\
\end{tabular} & -23.86 & 0 & -114.38 & 94.21 & -20.18 & 0 & \begin{tabular}{|c|}
-94.21 \\
\end{tabular} & 77.25 & -16.96 & 0 & \begin{tabular}{|l|}
-77.25 \\
\end{tabular} & \begin{tabular}{|c|}
63.09 \\
\end{tabular} & -14.16 \\
\hline 3100 & -166.23 & 138.24 & -27.99 & 3200 & -138.24 & 114.38 & -23.86 & 3300 & -114.38 & 94.21 & -20.18 & 3400 & -94.21 & 77.25 & -16.96 & 3500 & -77.25 & 63.09 & -14.16 \\
\hline 3200 & -66.23 & 138.24 & 72.01 & 3300 & -38.24 & 114.38 & 76.14 & 3400 & -14.38 & 94.21 & 79.82 & 3500 & 5.79 & 77.25 & 83.04 & 3600 & 22.75 & 63.09 & 85.84 \\
\hline 3300 & 33.77 & 38.24 & 72.01 & 3400 & 61.76 & 14.38 & 76.14 & 3500 & 85.62 & -5.79 & 79.82 & 3600 & 105.79 & -22.75 & 83.04 & 3700 & 122.75 & -36.91 & 85.84 \\
\hline Call: M & ay-06 & & & Call: M & ay-06 & & & Call: $M$ & $y-06$ & & & Call: Ma & $y-06$ & & & Call: $\mathbf{M}$ & $y-06$ & & \\
\hline $\mathrm{S}$ & & Payoff & & $\mathrm{S}$ & & Payoff & & $\mathrm{S}$ & & Payoff & & $\mathrm{S}$ & & Payoff & & $\mathrm{S}$ & & Payoff & \\
\hline & $\begin{array}{l}\text { Long } \\
3100\end{array}$ & $\begin{array}{l}\text { Short } \\
3200\end{array}$ & Total & & $\begin{array}{l}\text { Long } \\
3200\end{array}$ & $\begin{array}{l}\text { Short } \\
3300\end{array}$ & Total & & $\begin{array}{l}\text { Long } \\
3300\end{array}$ & $\begin{array}{l}\text { Short } \\
3400\end{array}$ & Total & & $\begin{array}{l}\text { Long } \\
3400\end{array}$ & $\begin{array}{l}\text { Short } \\
3500\end{array}$ & \begin{tabular}{|l|} 
Total \\
\end{tabular} & & $\begin{array}{l}\text { Long } \\
3500\end{array}$ & $\begin{array}{l}\text { Short } \\
3600\end{array}$ & Total \\
\hline 0 & -166.67 & 138.60 & -28.06 & 0 & -138.60 & 114.69 & -23.92 & 0 & -114.69 & 94.45 & -20.23 & 0 & $\begin{array}{l}-94.45 \\
\end{array}$ & 77.45 & -17.00 & 0 & \begin{tabular}{l|l}
-77.45 \\
\end{tabular} & $\begin{array}{ll}63.25 \\
\end{array}$ & -14.20 \\
\hline 3100 & -166.67 & 138.60 & -28.06 & 3200 & -138.60 & 114.69 & -23.92 & 3300 & -114.69 & 94.45 & -20.23 & 3400 & -94.45 & 77.45 & $-17.00 \mid$ & 3500 & -77.45 & 63.25 & -14.20 \\
\hline 3200 & -66.67 & 138.60 & 71.94 & 3300 & -38.60 & 114.69 & 76.08 & 3400 & -14.69 & 94.45 & 79.77 & 3500 & 5.55 & 77.45 & 83.00 & 3600 & 22.55 & 63.25 & 85.80 \\
\hline 3300 & 33.33 & 38.60 & 71.94 & 3400 & 61.40 & 14.69 & 76.08 & 3500 & 85.31 & -5.55 & 79.77 & 3600 & 105.55 & -22.55 & 83.00 & 3700 & 122.55 & -36.75 & 85.80 \\
\hline Call: Ju & -06 & & & all: J & -06 & & & Call: $\mathrm{Jt}$ & 06 & & & Call: J & & & & Call: $\mathrm{J}$ & $1-06$ & & \\
\hline $\mathrm{S}$ & & Payoff & & $\mathrm{S}$ & & Payoff & & $\mathrm{S}$ & & Payoff & & $\mathrm{S}$ & & Payoff & & $\mathrm{S}$ & & Payoff & \\
\hline & \begin{tabular}{|l|} 
Long \\
3100
\end{tabular} & $\begin{array}{l}\text { Short } \\
3200\end{array}$ & Total & & $\begin{array}{l}\text { Long } \\
3200\end{array}$ & $\begin{array}{l}\text { Short } \\
3300\end{array}$ & \begin{tabular}{|l|} 
Total \\
\end{tabular} & & $\begin{array}{l}\text { Long } \\
3300\end{array}$ & $\begin{array}{l}\text { Short } \\
3400\end{array}$ & \begin{tabular}{|l|} 
Total \\
\end{tabular} & & $\begin{array}{l}\text { Long } \\
3400\end{array}$ & $\begin{array}{l}\text { Short } \\
3500\end{array}$ & \begin{tabular}{|l|} 
Total \\
\end{tabular} & & $\begin{array}{l}\text { Long } \\
3500\end{array}$ & $\begin{array}{l}\text { Short } \\
3600\end{array}$ & \begin{tabular}{|l|} 
Total \\
\end{tabular} \\
\hline 0 & -167.14 & $\begin{array}{c}138.99 \\
\end{array}$ & $\mid-28.14$ & 0 & \begin{tabular}{|l|}
-138.99 \\
\end{tabular} & 115.01 & -23.99 & 0 & -115.01 & $\begin{array}{l}94.72 \\
\end{array}$ & -20.29 & 0 & \begin{tabular}{|c|}
-94.72 \\
\end{tabular} & \begin{tabular}{|c|}
77.67 \\
\end{tabular} & \begin{tabular}{|l|}
-17.05 \\
\end{tabular} & 0 & \begin{tabular}{|l|}
-77.67 \\
\end{tabular} & \begin{tabular}{|l|}
63.43 \\
\end{tabular} & -14.24 \\
\hline 3100 & -167.14 & 138.99 & -28.14 & 3200 & -138.99 & 115.01 & -23.99 & 3300 & -115.01 & 94.72 & -20.29 & 3400 & -94.72 & 77.67 & -17.05 & 3500 & -77.67 & 63.43 & -14.24 \\
\hline 3200 & -67.14 & 138.99 & 71.86 & 3300 & -38.99 & 115.01 & 76.01 & 3400 & -15.01 & 94.72 & 79.71 & 3500 & 5.28 & 77.67 & 82.95 & 3600 & 22.33 & 63.43 & 85.76 \\
\hline 3300 & 32.86 & 38.99 & 71.86 & 3400 & 61.01 & 15.01 & 76.01 & 3500 & 84.99 & -5.28 & 79.71 & 3600 & 105.28 & -22.33 & 82.95 & 3700 & 122.33 & -36.57 & 85.76 \\
\hline Call: Ju & $1-06$ & & & Call: Ju & $1-06$ & & & Call: $\mathbf{J t}$ & -06 & & & Call: Ju & & & & Call: Ju & & & \\
\hline $\mathrm{S}$ & & Payoff & & $\mathrm{S}$ & & Payoff & & $\mathrm{S}$ & & Payoff & & $S$ & & Payoff & & $\mathrm{S}$ & & Payoff & \\
\hline & \begin{tabular}{|l|} 
Long \\
3100 \\
\end{tabular} & $\begin{array}{l}\text { Short } \\
3200\end{array}$ & Total & & $\begin{array}{l}\text { Long } \\
3200\end{array}$ & $\begin{array}{l}\text { Short } \\
3300\end{array}$ & Total & & $\begin{array}{l}\text { Long } \\
3300\end{array}$ & $\begin{array}{l}\text { Short } \\
3400\end{array}$ & Total & & $\begin{array}{l}\text { Long } \\
3400\end{array}$ & $\begin{array}{l}\text { Short } \\
3500\end{array}$ & \begin{tabular}{|l|} 
Total \\
\end{tabular} & & $\begin{array}{l}\text { Long } \\
3500\end{array}$ & $\begin{array}{l}\text { Short } \\
3600\end{array}$ & \begin{tabular}{|l|} 
Total \\
\end{tabular} \\
\hline 0 & -167.56 & \begin{tabular}{|c|}
139.35 \\
\end{tabular} & -28.22 & $\overline{0}$ & -139.35 & $\begin{array}{l}115.30 \\
\end{array}$ & -24.05 & 0 & -115.30 & 94.96 & -20.34 & 0 & \begin{tabular}{|c|}
-94.96 \\
\end{tabular} & \begin{tabular}{|l|}
77.87 \\
\end{tabular} & \begin{tabular}{|l|}
-17.09 \\
\end{tabular} & 0 & \begin{tabular}{|c|}
-77.87 \\
\end{tabular} & 63.59 & -14.28 \\
\hline 3100 & -167.56 & 139.35 & -28.22 & 3200 & -139.35 & 115.30 & -24.05 & 3300 & -115.30 & 94.96 & -20.34 & 3400 & -94.96 & 77.87 & -17.09 & 3500 & -77.87 & 63.59 & -14.28 \\
\hline 3200 & -67.56 & 139.35 & 71.78 & 3300 & -39.35 & 115.30 & 75.95 & 3400 & -15.30 & 94.96 & 79.66 & 3500 & 5.04 & 77.87 & 82.91 & 3600 & 22.13 & 63.59 & 85.72 \\
\hline 3300 & 32.44 & 39.35 & 71.78 & 3400 & 60.65 & 15.30 & 75.95 & 3500 & 84.70 & -5.04 & 79.66 & 3600 & 105.04 & -22.13 & 82.91 & 3700 & 122.13 & -36.41 & 85.72 \\
\hline Call: $\mathbf{A t}$ & 06 & & & Call: & & & & Call: & & & & Call: & & & & Call: & $8-06$ & & \\
\hline S & & Payoff & & S & & Payoff & & S & & Payoff & & S & & Payoff & & $\mathrm{S}$ & & Payoff & \\
\hline & \begin{tabular}{|l|} 
Long \\
3100 \\
\end{tabular} & $\begin{array}{l}\text { Short } \\
3200\end{array}$ & Total & & $\begin{array}{l}\text { Long } \\
3200 \\
\end{array}$ & $\begin{array}{l}\text { Short } \\
3300\end{array}$ & \begin{tabular}{|l|} 
Total \\
\end{tabular} & & $\begin{array}{l}\text { Long } \\
\mathbf{3 3 0 0} \\
\end{array}$ & $\begin{array}{l}\text { Short } \\
3400\end{array}$ & \begin{tabular}{|l|} 
Total \\
\end{tabular} & & $\begin{array}{l}\text { Long } \\
3400 \\
\end{array}$ & $\begin{array}{l}\text { Short } \\
3500\end{array}$ & \begin{tabular}{|l|} 
Total \\
\end{tabular} & & $\begin{array}{l}\text { Long } \\
\mathbf{3 5 0 0} \\
\end{array}$ & $\begin{array}{l}\text { Short } \\
3600 \\
\end{array}$ & \begin{tabular}{|l|} 
Total \\
\end{tabular} \\
\hline 0 & -169.16 & 140.68 & -28.48 & 0 & -140.68 & 116.40 & -24.28 & 0 & -116.40 & 95.87 & -20.53 & 0 & $\begin{array}{l}-95.87 \\
\end{array}$ & 78.61 & -17.26 & 0 & \begin{tabular}{l|l|}
-78.61 \\
\end{tabular} & 64.20 & -14.41 \\
\hline 3100 & -169.16 & 140.68 & -28.48 & 3200 & -140.68 & 116.40 & -24.28 & 3300 & -116.40 & 95.87 & -20.53 & 3400 & -95.87 & 78.61 & -17.26 & 3500 & -78.61 & 64.20 & -14.41 \\
\hline 3200 & -69.16 & 140.68 & 71.52 & 3300 & -40.68 & 116.40 & 75.72 & 3400 & -16.40 & 95.87 & 79.47 & 3500 & 4.13 & 78.61 & 82.74 & 3600 & 21.39 & 64.20 & 85.59 \\
\hline 3300 & 30.84 & 40.68 & 71.52 & 3400 & 59.32 & 16.40 & 75.72 & 3500 & 83.60 & -4.13 & 79.47 & 3600 & 104.13 & -21.39 & 82.74 & 3700 & 121.39 & -35.80 & 85.59 \\
\hline
\end{tabular}


Table 7.6 continued
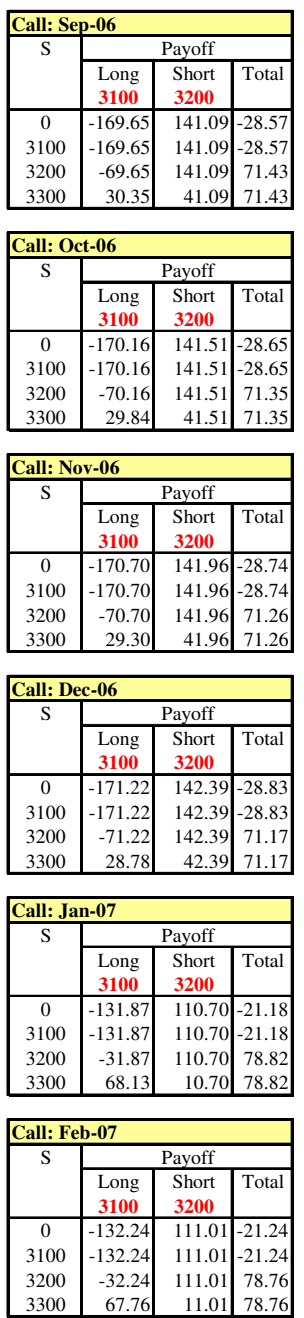

\begin{tabular}{|c|c|c|c|}
\hline \multicolumn{4}{|c|}{ Call: Mar-07 } \\
\hline \multirow[t]{2}{*}{$\mathrm{S}$} & \multicolumn{3}{|c|}{ Payoff } \\
\hline & $\begin{array}{l}\text { Long } \\
3100\end{array}$ & $\begin{array}{l}\text { Short } \\
3200\end{array}$ & Total \\
\hline$\overline{0}$ & -132.67 & 111.36 & -21.30 \\
\hline 3100 & -132.67 & 111.36 & -21.30 \\
\hline 3200 & -32.67 & 111.36 & 78.70 \\
\hline 3300 & 67.33 & 11.36 & 78.70 \\
\hline
\end{tabular}

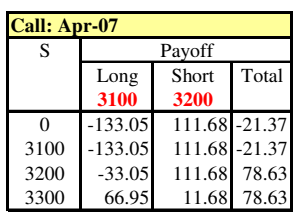

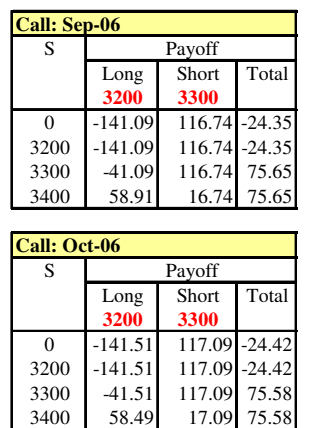
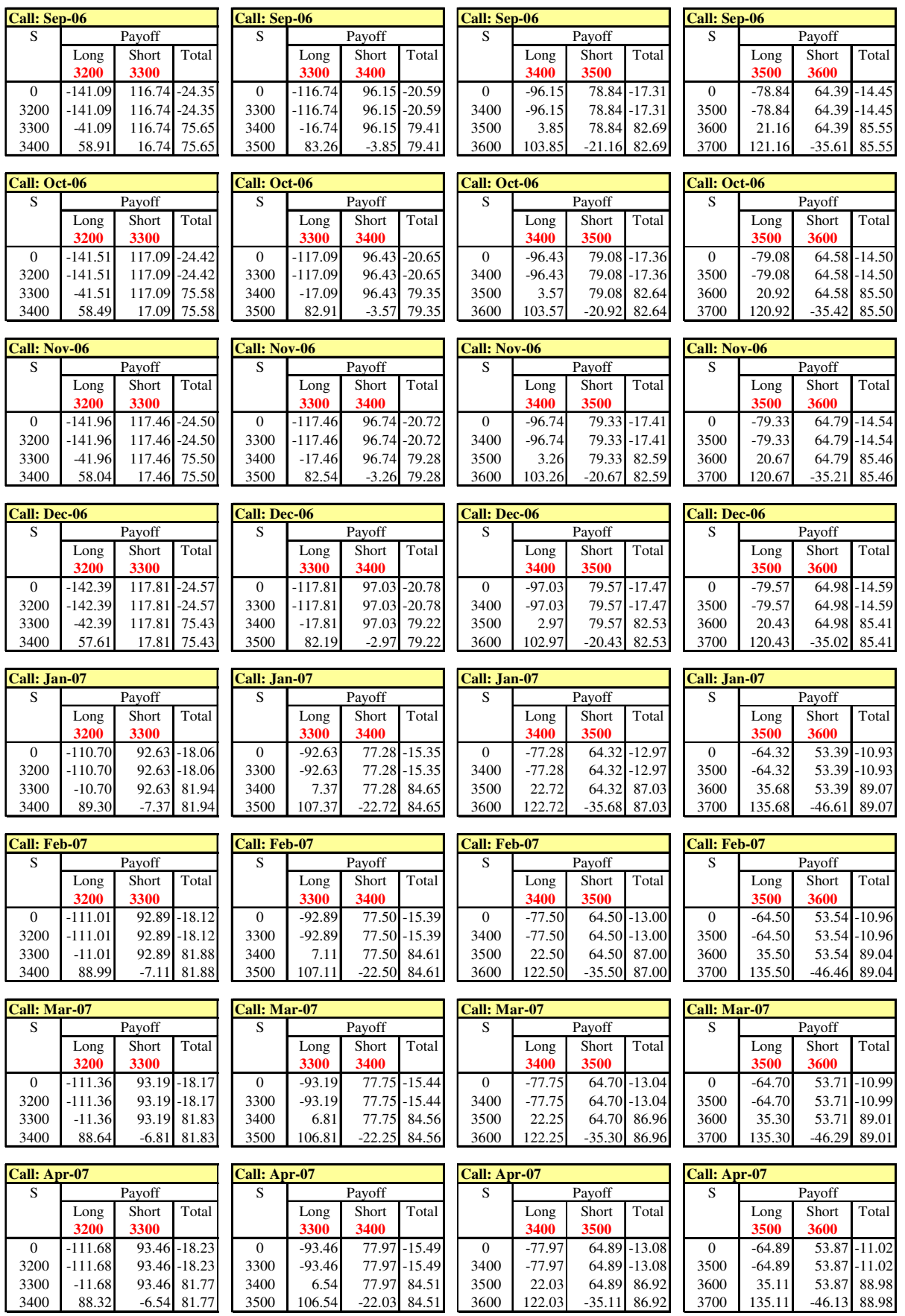
Table 7.6 continued
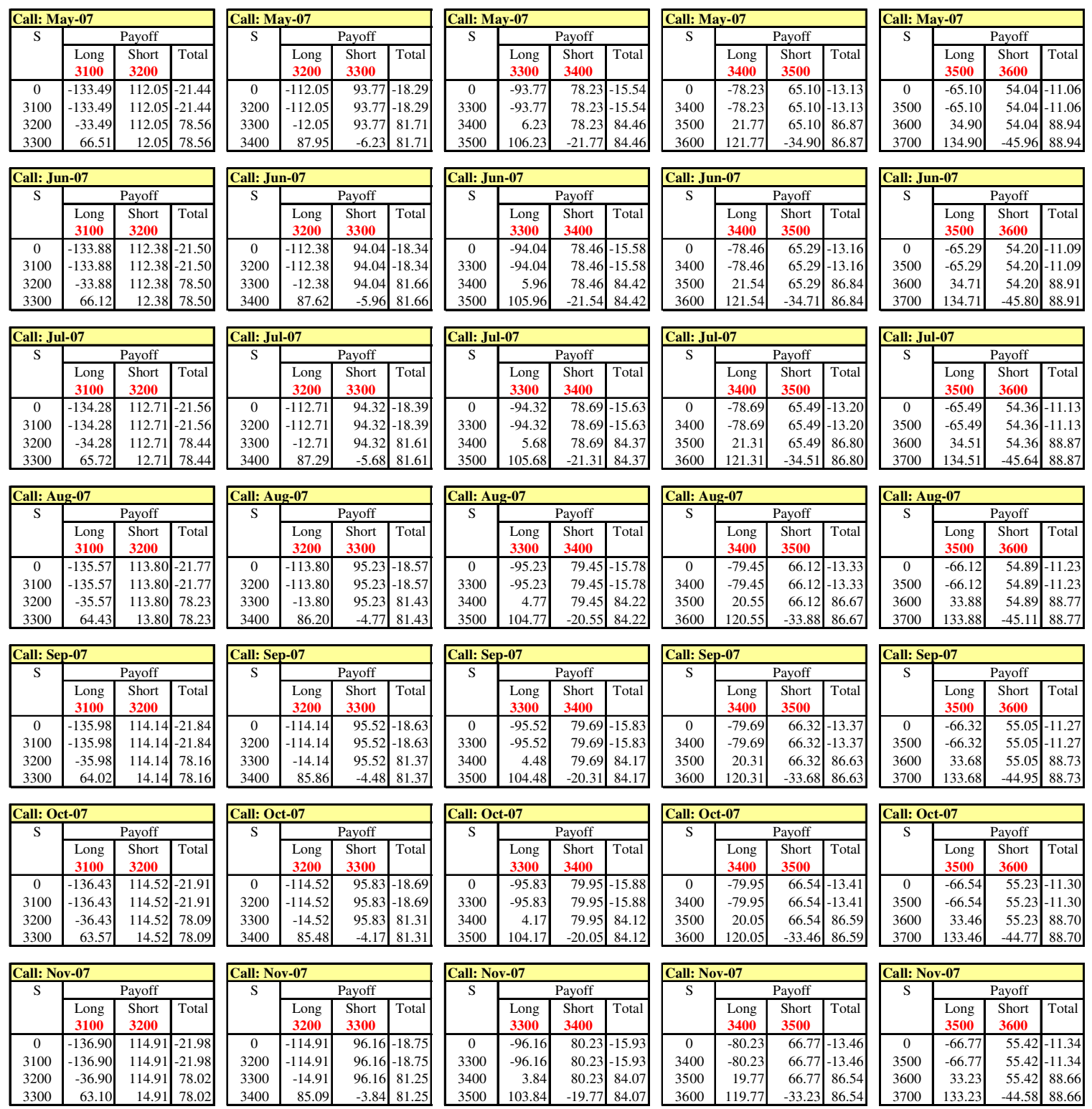

\begin{tabular}{|c|r|r|r|}
\hline \multicolumn{4}{|c|}{ Call: Nov-07 } \\
\hline \multirow{2}{*}{$S$} & \multicolumn{3}{|c|}{ Payoff } \\
\cline { 2 - 4 } & Long & Short & Total \\
& $\mathbf{3 2 0 0}$ & $\mathbf{3 3 0 0}$ & \\
\hline 0 & -114.91 & 96.16 & -18.75 \\
3200 & -114.91 & 96.16 & -18.75 \\
3300 & -14.91 & 96.16 & 81.25 \\
3400 & 85.09 & -3.84 & 81.25 \\
\hline
\end{tabular}

\begin{tabular}{|c|r|r|r|}
\hline \multicolumn{4}{|c|}{ Call: Nov-07 } \\
\hline \multirow{2}{*}{$\mathrm{S}$} & \multicolumn{3}{|c|}{ Payoff } \\
\cline { 2 - 4 } & Long & Short & Total \\
& $\mathbf{3 3 0 0}$ & $\mathbf{3 4 0 0}$ & \\
\hline 0 & -96.16 & 80.23 & -15.93 \\
3300 & -96.16 & 80.23 & -15.93 \\
3400 & 3.84 & 80.23 & 84.07 \\
3500 & 103.84 & -19.77 & 84.07 \\
\hline
\end{tabular}
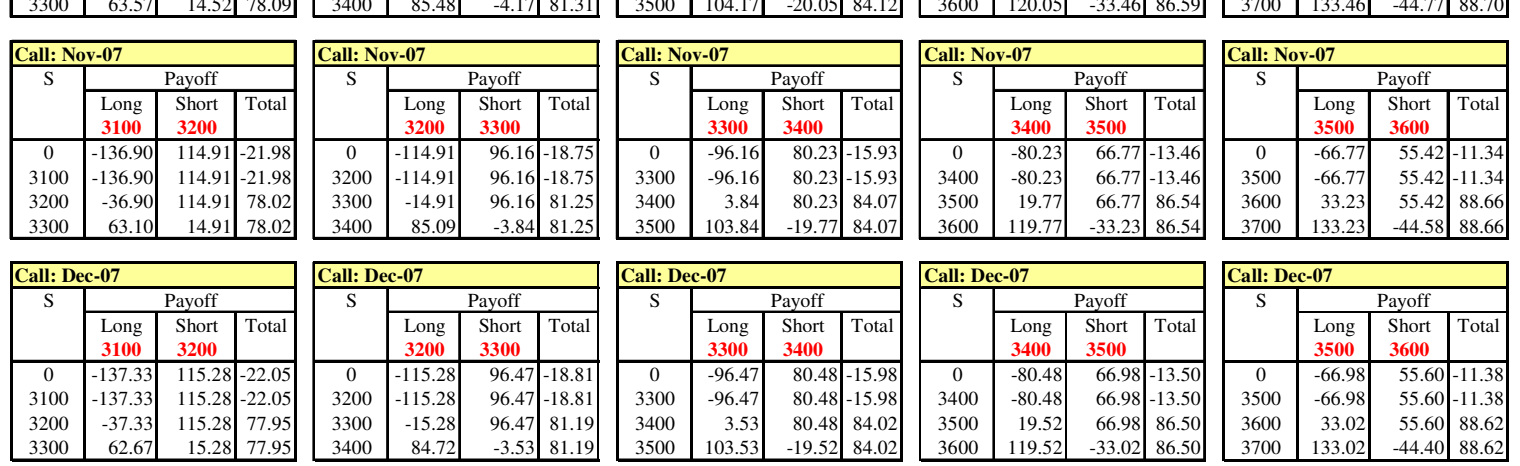


\section{Bull Spreads Using Put Options:}

Bull spreads can also be created by taking a long position on a put option with a specific strike price and a short position on a put option with a higher strike price. Both options have the same maturity date.

Table (7.11) demonstrates all possible bull spread strategies using put options for 2006 and 2007 based on the indications of 22 February 2005. There are 5 different spreads each month, so there are 120 bull spread strategies possible using put options for the 24 months between Jan-06 and Dec-07.

As seen from table (6.2) in chapter 6 , the minimum probability for monthly average copper prices in 2006 and 2007 to be greater than $\$ 2,700$ is between 0.95 and 1.00, which is close to 1 . The maximum value of put options' strike prices on the indications of 22 February 2005 is $\$ 2,700$. Therefore, all 120 bull spread strategies that are shown in table (7.11) would be profitable and useful on the valuation date of 22 February 2005. The most profitable strategy in 2006 is created by buying a put option at $\$ 2,600 / t$ and selling a put option at $\$ 2,700 / t$ for December 2006. The least profitable spread in 2006 is created by taking a long position in a put option at $\$ 2,200 / t$ and a short position in a put option at $\$ 2,300 /$ t. In 2007 , the best strategy is created by taking a long position at $\$ 2,600 / t$ and a short position at $\$ 2,700 / t$. The least profitable strategy in 2007 is created by buying a put option at $\$ 2,300 / \mathrm{t}$ and writing a put option at $\$ 2,400 / \mathrm{t}$.

The following tables and figures construct these four bull spread strategies involving put options. 
Bull Spread for January 2006 (minimum profit)

- Spot Price (22 Feb-05):

$\$ 3,367$

- Asian Put Options:

January-2006

- Positions:

Long Put @ 2200: $\quad$ premium $\$ 55.50$

Short Put @ 2300: $\quad$ premium $\$ 77.17$

Table 7.7: Profit from a Bull Spread (Jan-06 Put options, X: 2200/2300)

\begin{tabular}{cccc}
\hline $\begin{array}{c}\text { Copper price } \\
\text { range }\end{array}$ & $\begin{array}{c}\text { Profit from long } \\
\text { put @ 2200 }\end{array}$ & $\begin{array}{c}\text { Profit from short } \\
\text { put @ 2300 }\end{array}$ & $\begin{array}{c}\text { Total } \\
\text { profit }\end{array}$ \\
\hline $\mathrm{S}_{\mathrm{AVG}}>2300$ & -57.21 & 79.55 & 22.34 \\
$2300 \geq \mathrm{S}_{\mathrm{AVG}}>2200$ & -57.21 & $\mathrm{~S}_{\mathrm{AVG}}-2220.45$ & $\mathrm{~S}_{\mathrm{AVG}}-2277.66$ \\
$\mathrm{~S}_{\mathrm{AVG}} \leq 2200$ & $2142.79-\mathrm{S}_{\mathrm{AVG}}$ & $\mathrm{S}_{\mathrm{AVG}}-2220.45$ & -77.66 \\
\hline
\end{tabular}

If the average price of copper in January 2006 is greater than $\$ 2,300 /$ t, both bought and written put options would not be exercised, so the total payoff at the maturity would be the sum of the future values of initial payoffs (premium) $-\$ 57.21$ and $\$ 79.55$, which equals $\$ 22.34$. If the average price of copper is between $\$ 2,300 / t$ and $\$ 2,200 / t$, the put option that was bought at $\$ 2,200$ would not be exercised, but the written put option at $\$ 2,300$ would be exercised. In this case, the payoff from long position in a put option at $\$ 2,200$ would be negative $\$ 57.21$, and the payoff from short position in a put option at $\$ 2,300$ would be $\$ 79.55$ plus $\left\{\$ 2,300-\mathrm{S}_{\mathrm{AVG}}\right\}$, which equals $\left\{\mathrm{S}_{\mathrm{AVG}}-\$ 2,220.45\right\}$. At the maturity of the options, the total payoff would be $\left\{\mathrm{S}_{\mathrm{AVG}}-\$ 2,277.66\right\}$. If the average price of copper is less than $\$ 2,200$, both put options would be exercised, and the total payoff would result loss of $-\$ 77.66$. Profit is made when $\left[\mathrm{S}_{\mathrm{AVG}(\mathrm{Jan}-2006)}>\$ 2,277.66\right]$ and is maximum of $\$ 22.34$ when $\left[\mathrm{S}_{\mathrm{AVG}(\mathrm{Jan}-2006)}>\$ 2,300\right]$.

\section{Bull Spread for December 2006 (maximum profit)}

- Spot Price (22 Feb-05):

- Asian Put Options:

- Positions:
$\$ 3,367$

December-2006

LongPut@ 2600: premium \$162.76

Short Put@ 2700: $\quad$ premium \$205.42 
Table 7.8: Profit from a Bull Spread (Dec-06 Put options, X: 2600/2700)

\begin{tabular}{cccc}
\hline $\begin{array}{c}\text { Copper price } \\
\text { range }\end{array}$ & $\begin{array}{c}\text { Profit from long } \\
\text { put @ 2600 }\end{array}$ & $\begin{array}{c}\text { Profit from short } \\
\text { put @ 2700 }\end{array}$ & $\begin{array}{c}\text { Total } \\
\text { profit }\end{array}$ \\
\hline $\mathrm{S}_{\mathrm{AVG}}>2700$ & -174.18 & 219.83 & 45.65 \\
$2700 \geq \mathrm{S}_{\mathrm{AVG}}>2600$ & -174.18 & $\mathrm{~S}_{\mathrm{AVG}}-2480.17$ & $\mathrm{~S}_{\mathrm{AVG}}-2654.35$ \\
$\mathrm{~S}_{\mathrm{AVG}} \leq 2600$ & $2425.82-\mathrm{S}_{\mathrm{AVG}}$ & $\mathrm{S}_{\mathrm{AVG}}-2480.17$ & -54.35 \\
\hline
\end{tabular}

Profit is made when $\left[\mathrm{S}_{\mathrm{AVG}(\mathrm{Dec}-2006)}>\$ 2,654.35\right]$ and is maximum of $\$ 45.65$ when $\left[\mathrm{S}_{\mathrm{AVG}(\operatorname{Dec}-2006)}>\$ 2,700\right]$.

\section{Bull Spread for January 2007 (minimum profit)}

- Spot Price (22 Feb-05):

- Asian Put Options:

- Positions:
$\$ 3,367$

January-2007

Long Put@ 2300: $\quad$ premium $\$ 132.34$

Short Put @ 2400: $\quad$ premium \$162.34

Table 7.9: Profit from a Bull Spread (Jan-07 Put options, X: 2300/2400)

\begin{tabular}{cccc}
\hline $\begin{array}{c}\text { Copper price } \\
\text { range }\end{array}$ & $\begin{array}{c}\text { Profit from long } \\
\text { put @ 2300 }\end{array}$ & $\begin{array}{c}\text { Profit from short } \\
\text { put @ 2400 }\end{array}$ & $\begin{array}{c}\text { Total } \\
\text { profit }\end{array}$ \\
\hline $\mathrm{S}_{\mathrm{AVG}}>2400$ & -142.05 & 174.25 & 32.20 \\
$2400 \geq \mathrm{S}_{\mathrm{AVG}}>2300$ & -142.05 & $\mathrm{~S}_{\mathrm{AVG}}-2225.75$ & $\mathrm{~S}_{\mathrm{AVG}}-2367.80$ \\
$\mathrm{~S}_{\mathrm{AVG}} \leq 2300$ & $2157.95-\mathrm{S}_{\mathrm{AVG}}$ & $\mathrm{S}_{\mathrm{AVG}}-2225.75$ & -67.80 \\
\hline
\end{tabular}

Profit is made when $\left[\mathrm{S}_{\mathrm{AVG}(\mathrm{Jan}-2007)}>\$ 2,367.80\right]$ and is maximum of $\$ 32.20$ when

$\left[\mathrm{S}_{\mathrm{AVG}(\mathrm{Jan}-2007)}>\$ 2,400\right]$.

\section{Bull Spread for December 2007 (maximum profit)}

- Spot Price (22 Feb-05):

- Asian Put Options:

- Positions:
$\$ 3,367$

December-2007

Long Put @ 2600: $\quad$ premium $\$ 250.43$

Short Put @ 2700: premium $\$ 302.15$

Table 7.10: Profit from a Bull Spread (Dec-07 Put options, X: 2600/2700)

\begin{tabular}{cccc}
\hline $\begin{array}{c}\text { Copper price } \\
\text { range }\end{array}$ & $\begin{array}{c}\text { Profit from long } \\
\text { put @ 2600 }\end{array}$ & $\begin{array}{c}\text { Profit from short } \\
\text { put @ 2700 }\end{array}$ & $\begin{array}{c}\text { Total } \\
\text { profit }\end{array}$ \\
\hline $\mathrm{S}_{\mathrm{AVG}}>2700$ & -279.93 & 337.75 & 57.82 \\
$2700 \geq \mathrm{S}_{\mathrm{AVG}}>2600$ & -279.93 & $\mathrm{~S}_{\mathrm{AVG}}-2362.25$ & $\mathrm{~S}_{\mathrm{AVG}}-2642.18$ \\
$\mathrm{~S}_{\mathrm{AVG}} \leq 2600$ & $2320.07-\mathrm{S}_{\mathrm{AVG}}$ & $\mathrm{S}_{\mathrm{AVG}}-2362.25$ & -42.18 \\
\hline
\end{tabular}


Profit is made when $\left[\mathrm{S}_{\mathrm{AVG}(\mathrm{Dec}-2007)}>\$ 2,642.18\right]$ and is maximum of $\$ 57.82$ when $\left[\mathrm{S}_{\mathrm{AVG}(\operatorname{Dec}-2007)}>\$ 2,700\right]$.

Figures (7.6) though (7.9) illustrate profits from bull spread strategies involving put options.

Figure (7.6): Bull Spread Using Put Options Jan-06, X: 2200/2300

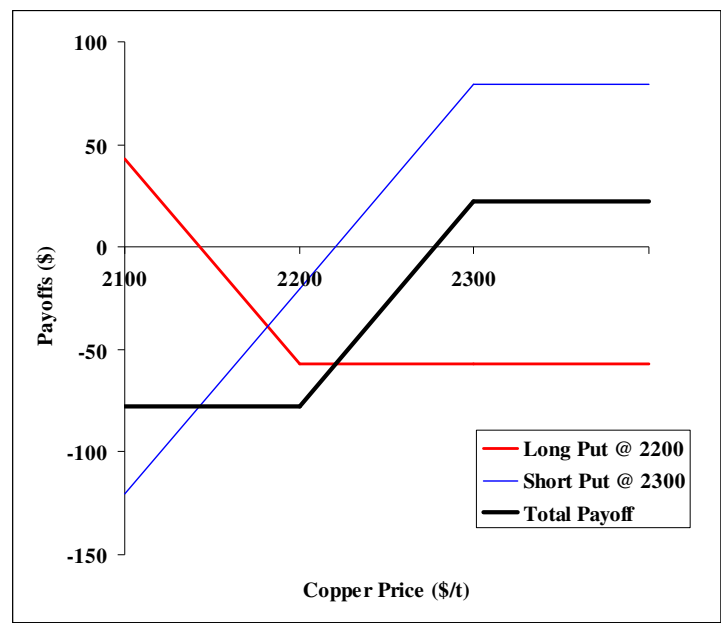

Figure (7.7): Bull Spread Using Put Options Dec-06, X: 2600/2700

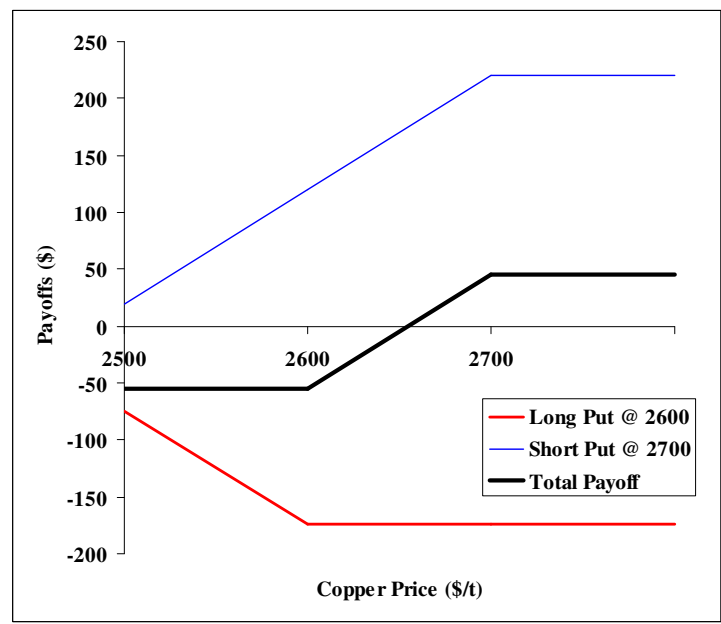

Figure (7.8): Bull Spread Using Put Options Jan-07, X: 2300/2400

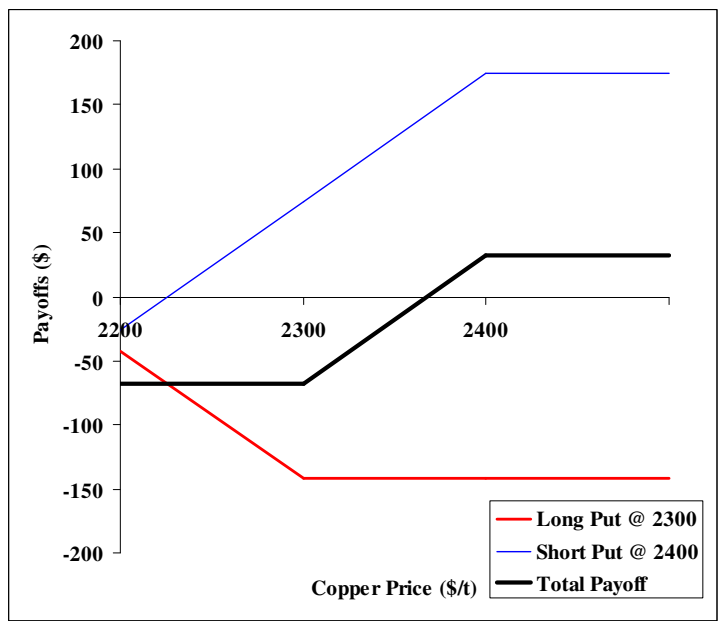

Figure (7.9): Bull Spread Using Put Options Dec-07, X: 2600/2700

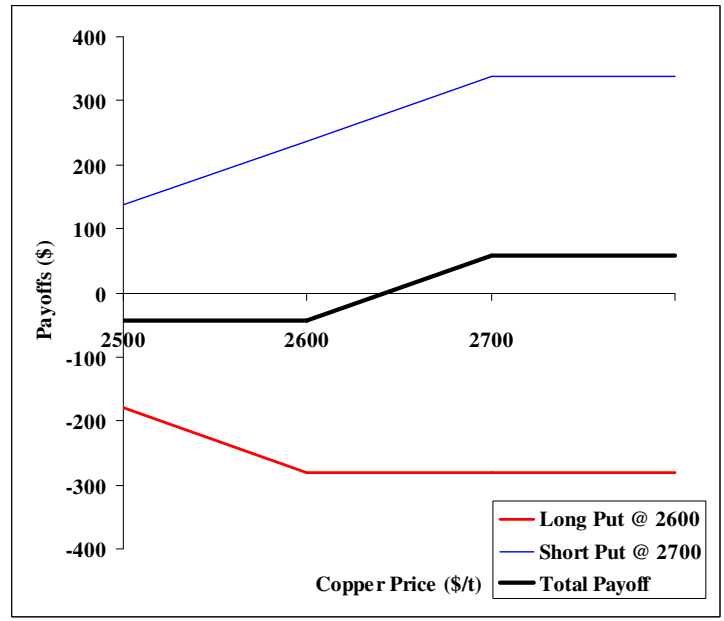


Table 7.11: Bull Spreads Using Put Options for 2006 and 2007
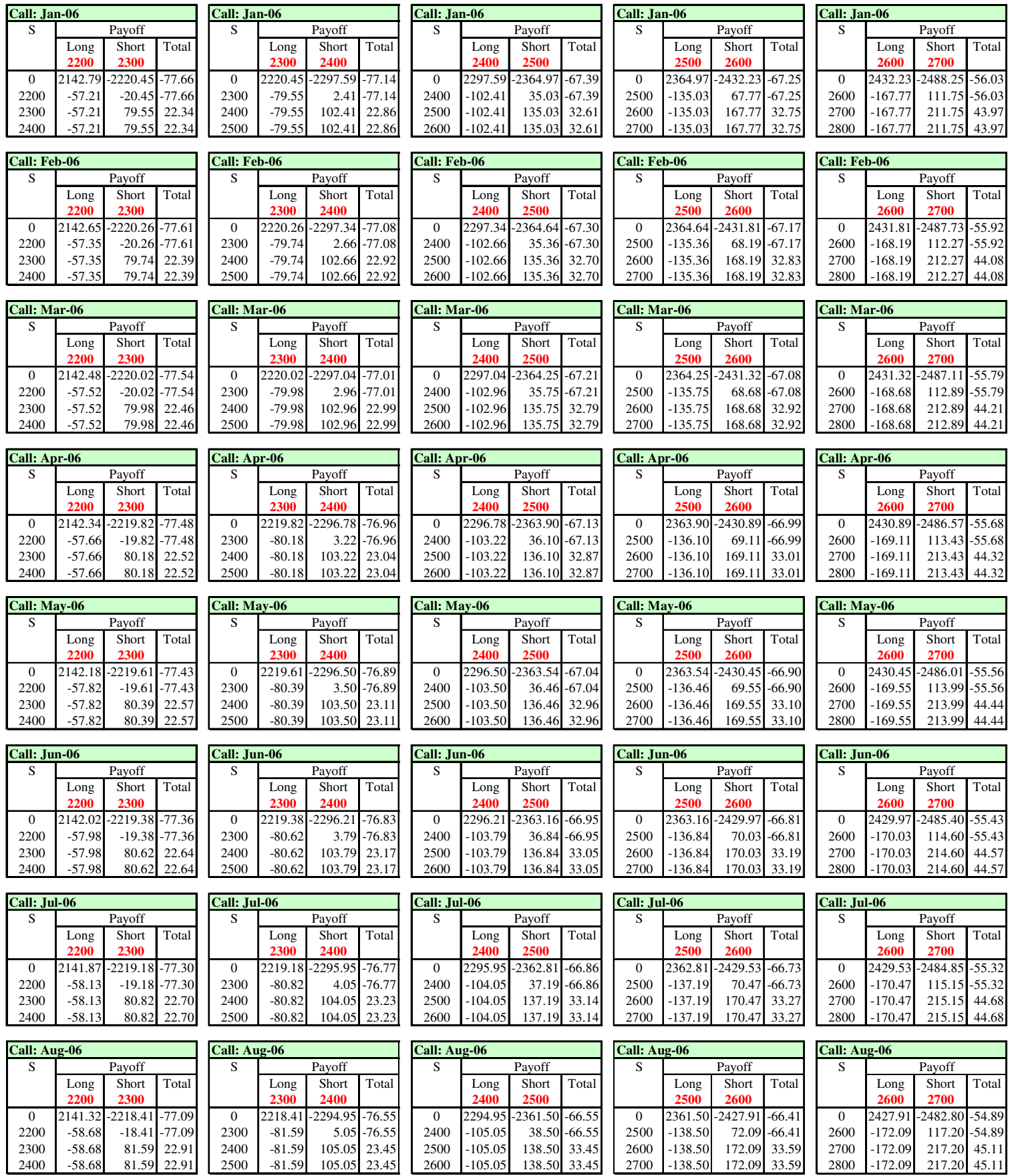
Table 7.11 continued
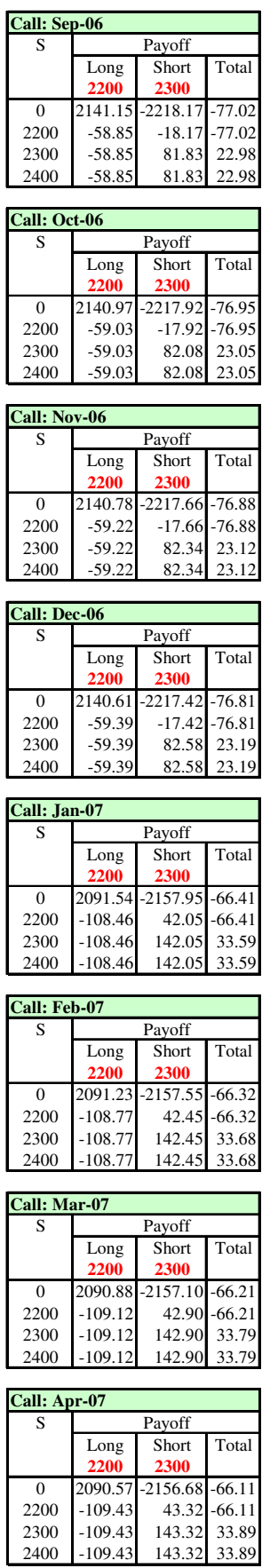
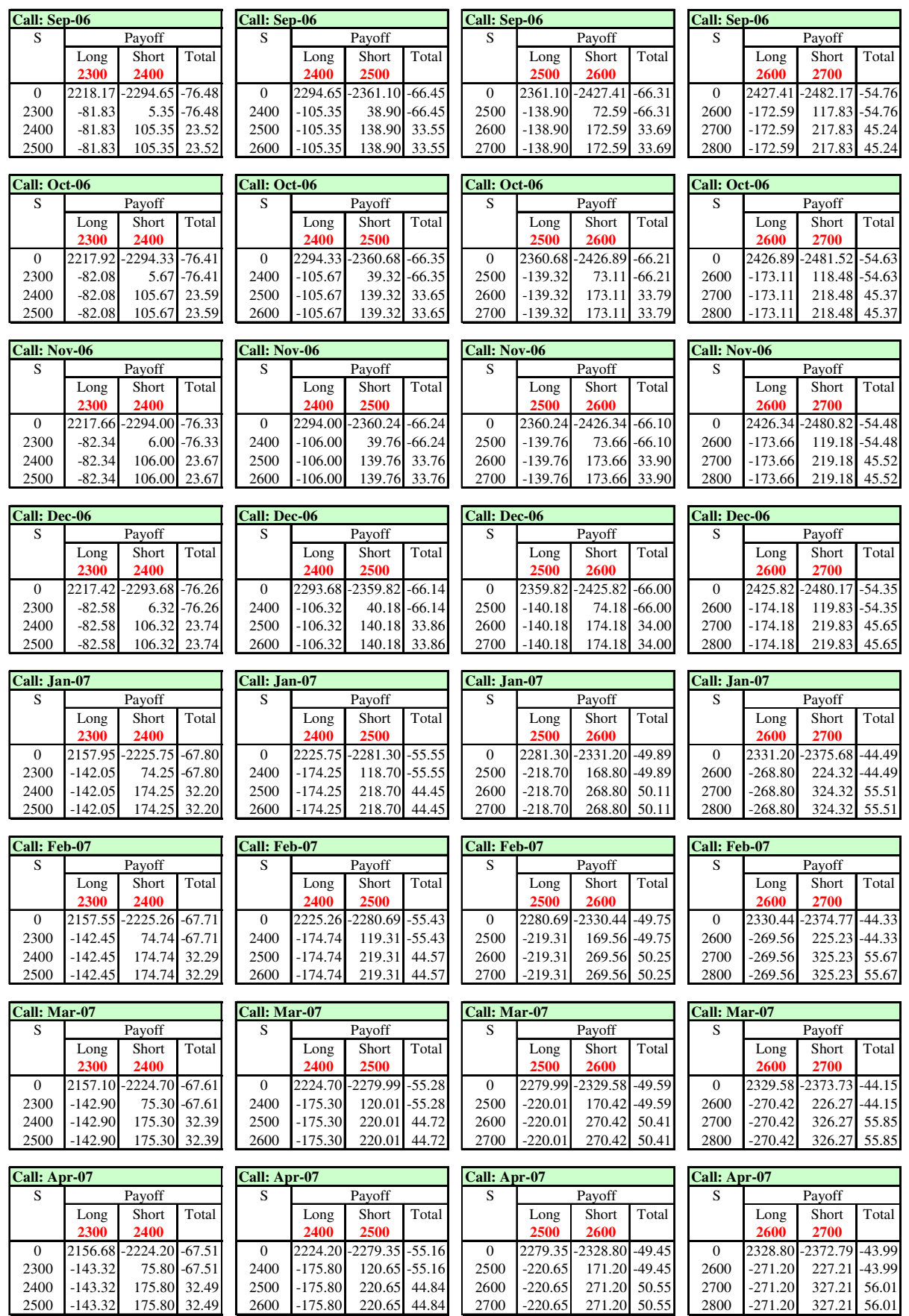
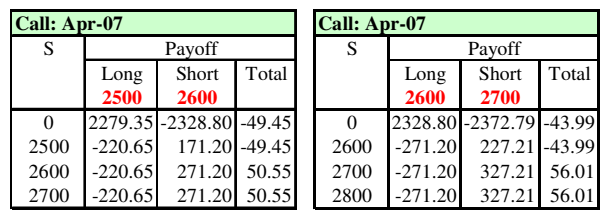
Table 7.11 continued
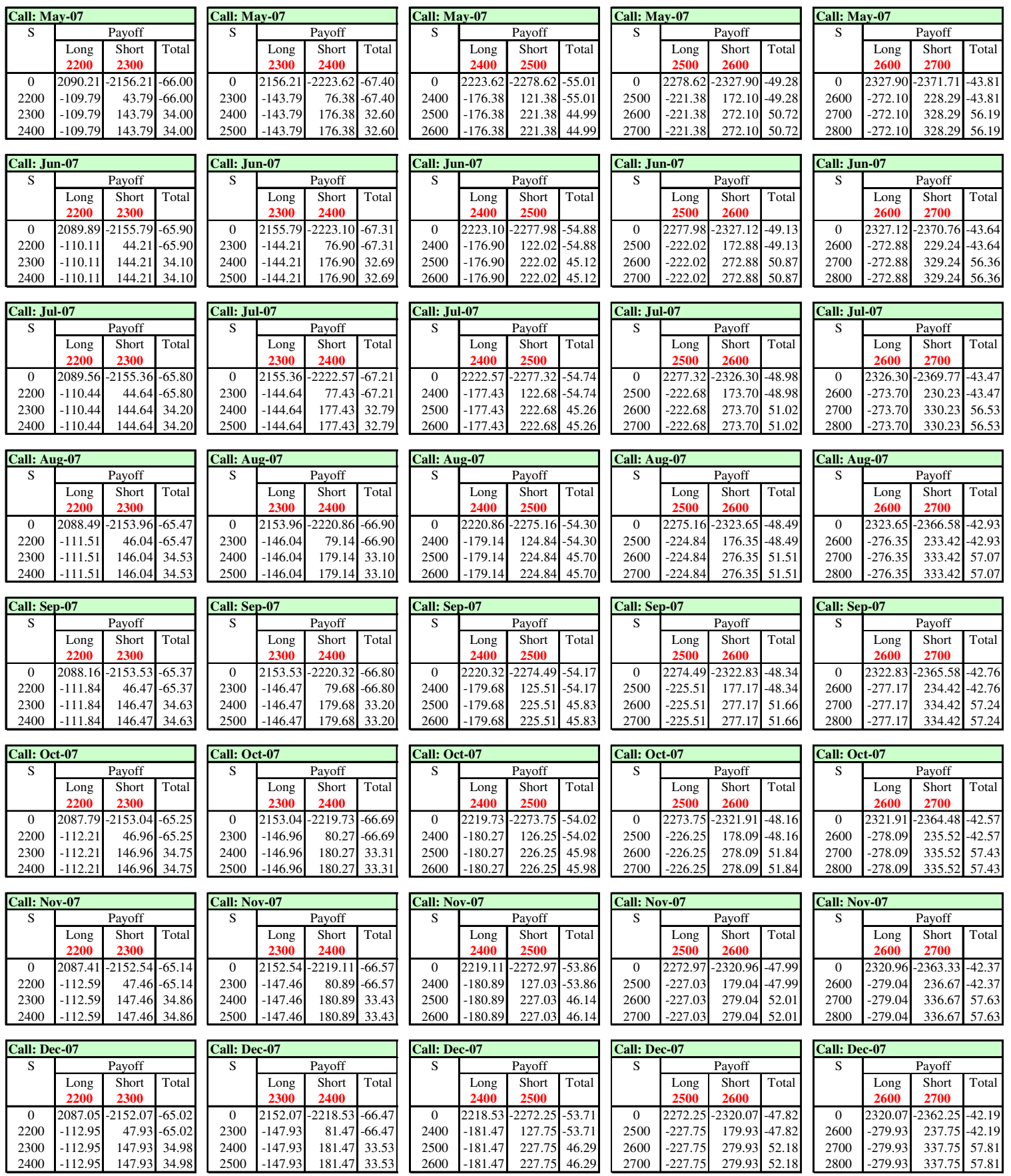

In conclusion, bull spread strategies using both call and put options limit the investor's upside as well as the downside price risk. 


\subsubsection{BEAR SPREADS:}

If the underlying price is expected to decrease, a bear spread is a useful strategy. Therefore, bear spreads are the reverse strategies of bull spreads and they are used by option traders and commodity producers. Bear spread can be created by buying a call option with a specific strike price, and writing a call option with a lower strike price or buying a put option with a specific strike price, and writing a put option with a lower strike price. Both options have the same maturity date.

\section{Bear Spreads Using Call Options:}

Table (7.16) demonstrates all possible bear spread strategies using call options for 2006 and 2007 based on the indications of 22 February 2005. There are 5 different spreads each month, so in total there are 120 bear spread strategies developed using call options for 24 months between Jan-06 and Dec-07. As can be seen from table (6.2), monthly copper prices in 2006 are likely to be around $\$ 3,300 / t$ or less. In this case, bear spread can be created using call option with strike prices $(\$ 3,300, \$ 3,400)$; $(\$ 3,400$, $\$ 3,500)$ or $(\$ 3,500, \$ 3,600)$. Therefore, based on the analysis in chapter 6 , the best bear spread strategies involving call options might be in the months between Jan-06 and Dec07 as follows:

a) In 2006:

- Long position in a call option at $\$ 3,400 / \mathrm{t}$ and short position in a call option at $\$ 3,300 / t$ for January 2006 through December 2006.

- Long position in a call option at $\$ 3,500 / t$ and short position in a call option at \$3,400/t for January 2006 through December 2006. 
- Long position in a call option at $\$ 3,600 / t$ and short position in a call option at $\$ 3,500 /$ f for January 2006 through December 2006 .

Totally 36 bear spread strategies using call options might be potentially profitable.

b) In 2007:

The probabilities for monthly copper prices to be less than $\$ 3,500$ in 2007 are not greater than 0.50 , according to the analysis in chapter 6 . Thus, all bear spreads using call options in 2007 may not be optimal to be used because bear spreads are profitable when monthly average copper price is equal or less than $\$ 3,500 /$ t. The following tables construct four different bear spreads which have maximum and minimum profits.

\section{Bear Spread for December 2006 (maximum profit)}

- Spot Price (22 Feb-05):

- Asian Call Options:

- Positions:
$\$ 3,367$

December-2006

Long Call @ 3400: $\quad$ premium $\$ 90.67$

Short Call @ 3300: $\quad$ premium $\$ 110.09$

Table 7.12: Profit from a Bear Spread (Dec-06 Call options, X: 3400/3300)

\begin{tabular}{cccc}
\hline $\begin{array}{c}\text { Copper price } \\
\text { range }\end{array}$ & $\begin{array}{c}\text { Profit from long } \\
\text { call @ 3400 }\end{array}$ & $\begin{array}{c}\text { Profit from short } \\
\text { call @ 3300 }\end{array}$ & $\begin{array}{c}\text { Total } \\
\text { profit }\end{array}$ \\
\hline $\mathrm{S}_{\mathrm{AVG}}>3400$ & $\mathrm{~S}_{\mathrm{AVG}}-3497.03$ & $3417.81-\mathrm{S}_{\mathrm{AVG}}$ & -79.22 \\
$3400 \geq \mathrm{S}_{\mathrm{AVG}}>3300$ & -97.03 & $3417.81-\mathrm{S}_{\mathrm{AVG}}$ & $3320.78-\mathrm{S}_{\mathrm{AVG}}$ \\
$\mathrm{S}_{\mathrm{AVG}} \leq 3300$ & -97.03 & 117.81 & 20.78 \\
\hline
\end{tabular}

If monthly average copper price is greater than higher strike price of $\$ 3,400$, both bought and written call options would be exercised and this finally results $\$ 79.22$ of loss.

If the average price is between $\$ 3,400 / t$ and $\$ 3,300 / t$, a long call position would result loss, which equals the future value of the call premium. The total profit would be $\left\{3320.78-S_{A V G}\right\}$ in this case. If the average price is less than lower strike of $\$ 3,300$, both options would not be exercised, and this finally results $\$ 20.78$ of profit. 
Profit is made when $\left[\mathrm{S}_{\mathrm{AVG}(\mathrm{Dec}-2006)}<\$ 3,320.78\right]$ and is maximum of $\$ 20.78$ when $\left[\mathrm{S}_{\mathrm{AVG}(\text { Dec-2006) }} \leq \$ 3,300\right]$.

\section{Bear Spread for December 2006 (maximum profit)}

- Spot Price (22 Feb-05):

- Asian Call Options:

- Positions:

\section{$\$ 3,367$}

December-2006

Long Call @ 3500:

Short Call @ 3400: premium $\$ 74.35$

premium $\$ 90.67$

Table 7.13: Profit from a Bear Spread (Dec-06 Call options, X: 3500/3400)

\begin{tabular}{cccc}
\hline $\begin{array}{c}\text { Copper price } \\
\text { range }\end{array}$ & $\begin{array}{c}\text { Profit from long } \\
\text { call @ 3500 }\end{array}$ & $\begin{array}{c}\text { Profit from short } \\
\text { call @ 3400 }\end{array}$ & $\begin{array}{c}\text { Total } \\
\text { profit }\end{array}$ \\
\hline $\mathrm{S}_{\mathrm{AVG}}>3500$ & $\mathrm{~S}_{\mathrm{AVG}}-3579.57$ & $3497.03-\mathrm{S}_{\mathrm{AVG}}$ & -82.54 \\
$3500 \geq \mathrm{S}_{\mathrm{AVG}}>3400$ & -79.57 & $3497.03-\mathrm{S}_{\mathrm{AVG}}$ & $3417.46-\mathrm{S}_{\mathrm{AVG}}$ \\
$\mathrm{S}_{\mathrm{AVG}} \leq 3400$ & -79.57 & 97.03 & 17.46 \\
\hline
\end{tabular}

Profit is made when $\left[\mathrm{S}_{\mathrm{AVG}(\mathrm{Dec}-2006)}<\$ 3,417.46\right]$ and is maximum of $\$ 17.46$ when $\left[\mathrm{S}_{\mathrm{AVG}(\text { Dec-2006) }} \leq \$ 3,400\right]$.

\section{Bear Spread for December 2006 (maximum profit)}

- Spot Price (22 Feb-05):

- Asian Call Options:

- Positions:
$\$ 3,367$

December-2006

Long Call @ 3600: $\quad$ premium $\$ 60.72$

Short Call @ 3500: $\quad$ premium $\$ 74.35$

Table 7.14: Profit from a Bear Spread (Dec-06 Call options, X: 3600/3500)

\begin{tabular}{cccc}
\hline $\begin{array}{c}\text { Copper price } \\
\text { range }\end{array}$ & $\begin{array}{c}\text { Profit from long } \\
\text { call @ 3600 }\end{array}$ & $\begin{array}{c}\text { Profit from short } \\
\text { call @ 3500 }\end{array}$ & $\begin{array}{c}\text { Total } \\
\text { profit }\end{array}$ \\
\hline $\mathrm{S}_{\mathrm{AVG}}>3600$ & $\mathrm{~S}_{\mathrm{AVG}}-3664.98$ & $3579.57-\mathrm{S}_{\mathrm{AVG}}$ & -85.41 \\
$3600 \geq \mathrm{S}_{\mathrm{AVG}}>3500$ & -64.98 & $3579.57-\mathrm{S}_{\mathrm{AVG}}$ & $3514.59-\mathrm{S}_{\mathrm{AVG}}$ \\
$\mathrm{S}_{\mathrm{AVG}} \leq 3500$ & -64.98 & 79.57 & 14.59 \\
\hline
\end{tabular}

Profit is made when $\left[\mathrm{S}_{\mathrm{AVG}(\mathrm{Dec}-2006)}<\$ 3,514.59\right]$ and is maximum of $\$ 14.59$ when $\left[\mathrm{S}_{\mathrm{AVG}(\text { Dec-2006) }} \leq \$ 3,500\right]$. 
Bear Spread for January 2006 (minimum profit)

- Spot Price (22 Feb-05):

$\$ 3,367$

- Asian Call Options:

January-2006

- Positions:

Long Call @ 3600: $\quad$ premium $\$ 60.72$

Short Call @ 3500: $\quad$ premium $\$ 74.35$

Table 7.15: Profit from a Bear Spread (Jan-06 Call options, X: 3600/3500)

\begin{tabular}{cccc}
\hline $\begin{array}{c}\text { Copper price } \\
\text { range }\end{array}$ & $\begin{array}{c}\text { Profit from long } \\
\text { call @ } 3600\end{array}$ & $\begin{array}{c}\text { Profit from short } \\
\text { call @ 3500 }\end{array}$ & $\begin{array}{c}\text { Total } \\
\text { profit }\end{array}$ \\
\hline $\mathrm{S}_{\mathrm{AVG}}>3600$ & $\mathrm{~S}_{\mathrm{AVG}}-3662.59$ & $3576.64-\mathrm{S}_{\mathrm{AVG}}$ & -85.95 \\
$3600 \geq \mathrm{S}_{\mathrm{AVG}}>3500$ & -62.59 & $3576.64-\mathrm{S}_{\mathrm{AVG}}$ & $3514.05-\mathrm{S}_{\mathrm{AVG}}$ \\
$\mathrm{S}_{\mathrm{AVG}} \leq 3500$ & -62.59 & 76.64 & 14.05 \\
\hline
\end{tabular}

Profit is made when $\left[\mathrm{S}_{\mathrm{AVG}(\mathrm{Jan}-2006)}<\$ 3,514.05\right]$ and is maximum of $\$ 14.05$ when $\left[\mathrm{S}_{\mathrm{AVG}(\mathrm{Jan}-2006)} \leq \$ 3,500\right]$.

Bear spreads, created from call options will have an initial cash inflow because a call that is sold always has a greater value than a call which is bought.

Figure (7.10): Bear Spread Using Call Options Dec-06, X: 3400/3300

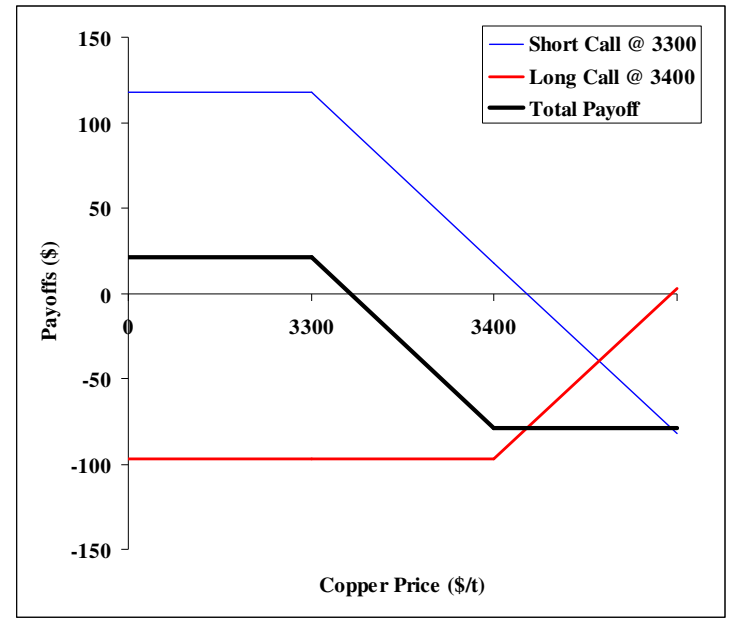

Figure (7.11): Bear Spread Using Call Options Dec-06, X: 3500/3400

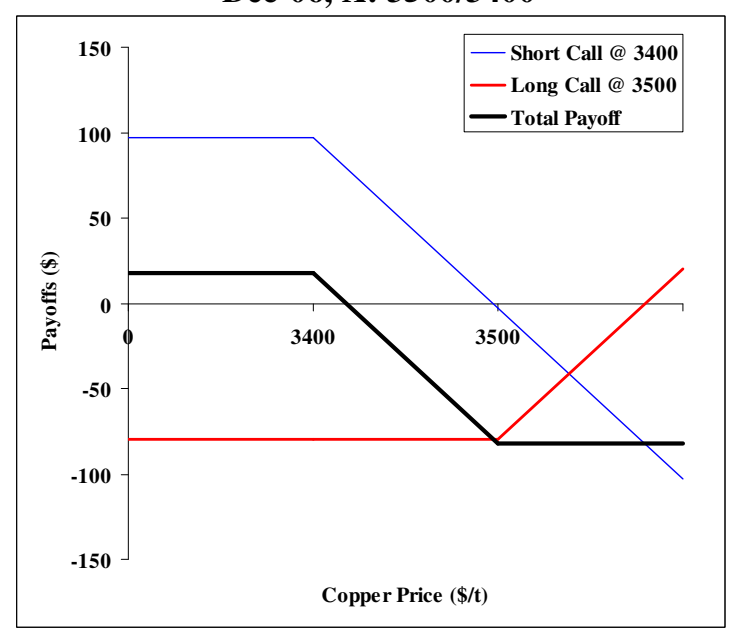


Figure (7.12): Bear Spread Using Call Options Dec-06, X: 3600/3500

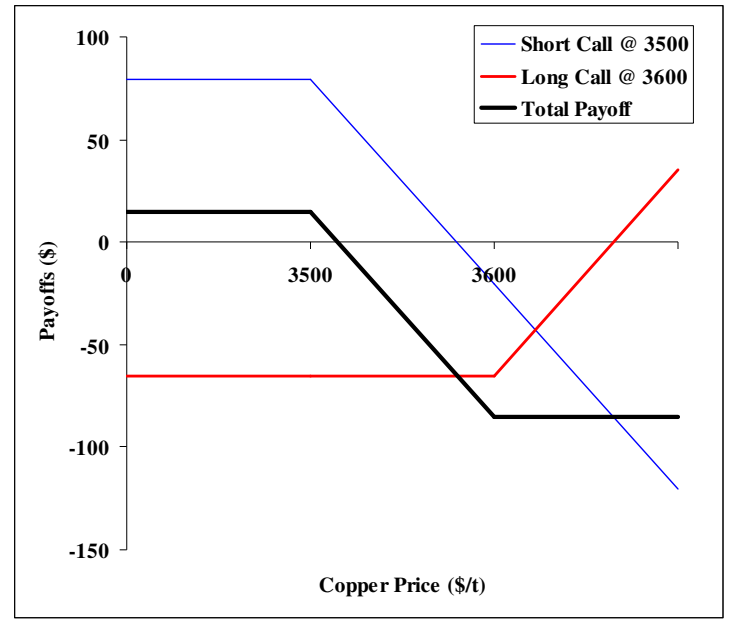

Figure (7.13): Bear Spread Using Call Options Jan-06, X: 3600/3500

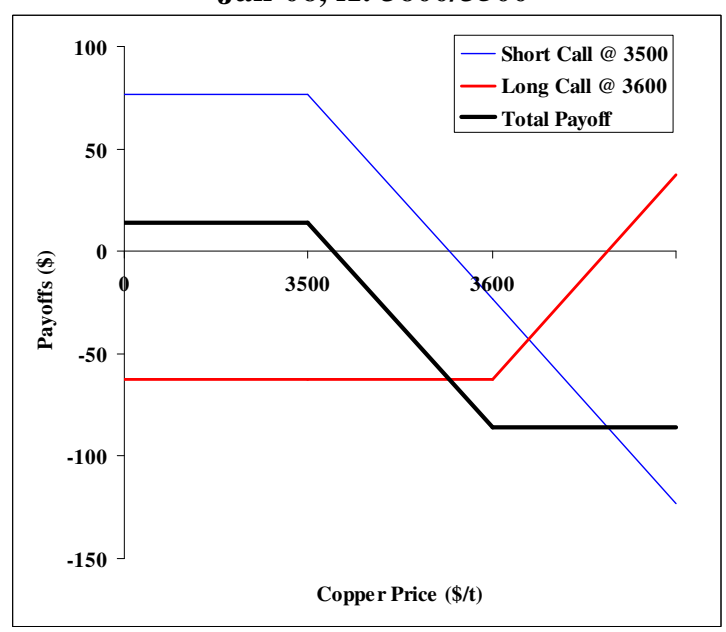


Table 7.16: Bear Spreads Using Call Options for 2006 and 2007
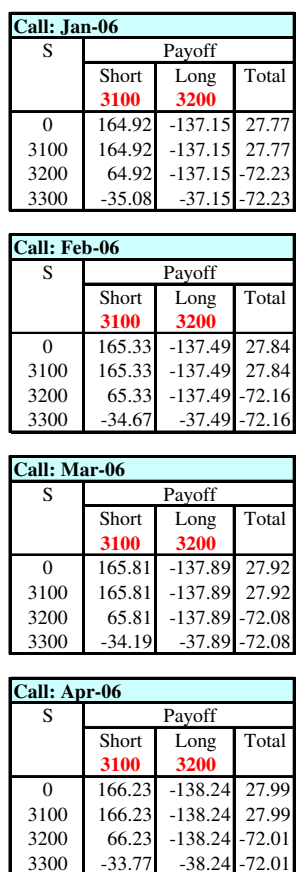

Call: May-06

\begin{tabular}{|c|r|r|r|}
\hline \multicolumn{4}{|c|}{ Call: May-06 } \\
\hline \multirow{2}{*}{$\mathrm{S}$} & \multicolumn{3}{|c|}{ Payoff } \\
\cline { 2 - 4 } & Short & Long & Total \\
& $\mathbf{3 1 0 0}$ & $\mathbf{3 2 0 0}$ & \\
\hline 0 & 166.67 & -138.60 & 28.06 \\
3100 & 166.67 & -138.60 & 28.06 \\
3200 & 66.67 & -138.60 & -71.94 \\
3300 & -33.33 & -38.60 & -71.94 \\
\hline
\end{tabular}

\begin{tabular}{|c|r|r|r|}
\hline \multicolumn{4}{|c|}{ Call: Jun-06 } \\
\hline \multirow{2}{*}{$\mathrm{S}$} & \multicolumn{3}{|c|}{ Payoff } \\
\cline { 2 - 4 } & Short & \multicolumn{1}{c|}{ Long } & Total \\
& \multicolumn{1}{|c|}{$\mathbf{1 1 0 0}$} & $\mathbf{3 2 0 0}$ & \\
\hline 0 & 167.14 & -138.99 & 28.14 \\
3100 & 167.14 & -138.99 & 28.14 \\
3200 & 67.14 & -138.99 & -71.86 \\
3300 & -32.86 & -38.99 & -71.86 \\
\hline
\end{tabular}

Call: Jul-06

\begin{tabular}{|c|r|r|r|}
\hline \multicolumn{4}{|c|}{ Call: Jul-06 } \\
\cline { 2 - 4 } & \multicolumn{3}{|c|}{ Payoff } \\
\cline { 2 - 4 } & \multicolumn{1}{|c|}{ Short } & Long & Total \\
\hline 0 & 167.56 & -139.35 & 28.22 \\
3100 & 167.56 & -139.35 & 28.22 \\
3200 & 67.56 & -139.35 & -71.78 \\
3300 & -32.44 & -39.35 & -71.78 \\
\hline
\end{tabular}

\begin{tabular}{|c|r|r|r|}
\hline \multicolumn{5}{|c|}{ Call: Aug-06 } \\
\hline \multirow{2}{*}{$\mathrm{S}$} & \multicolumn{3}{|c|}{ Payoff } \\
\cline { 2 - 4 } & Short & \multicolumn{1}{|c|}{ Long } & Total \\
& $\mathbf{3 1 0 0}$ & $\mathbf{3 2 0 0}$ & \\
\hline 0 & 169.16 & -140.68 & 28.48 \\
3100 & 169.16 & -140.68 & 28.48 \\
3200 & 69.16 & -140.68 & -71.52 \\
3300 & -30.84 & -40.68 & -71.52 \\
\hline
\end{tabular}
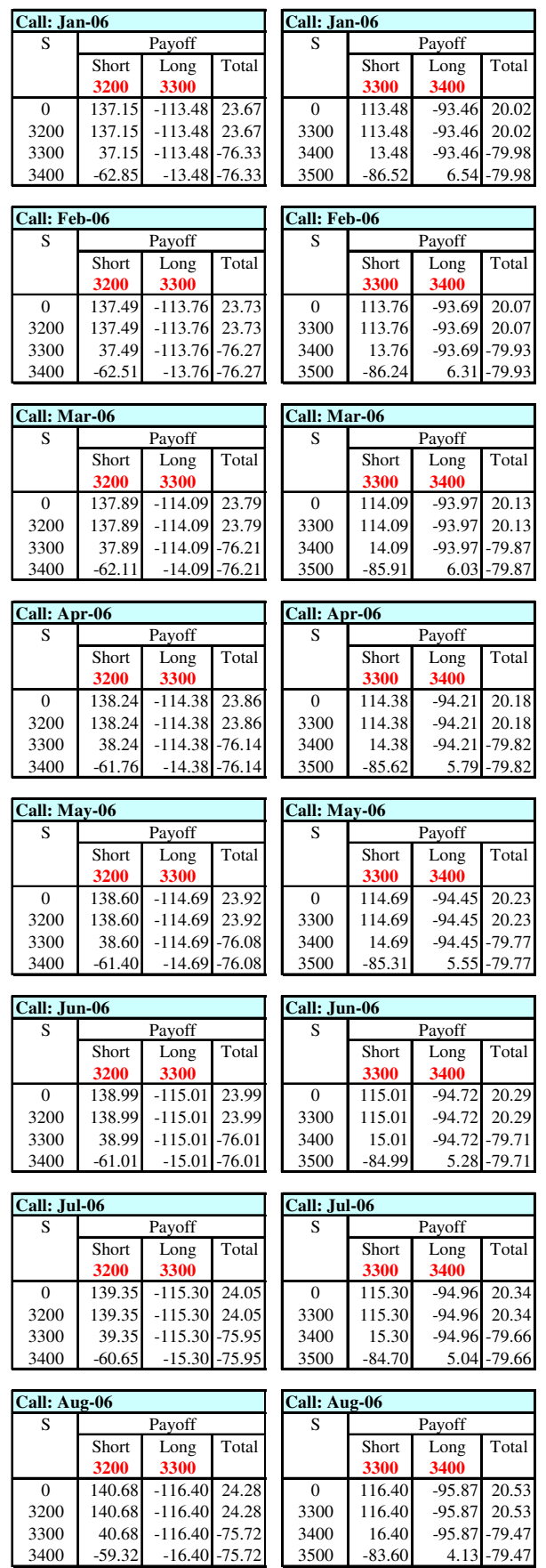
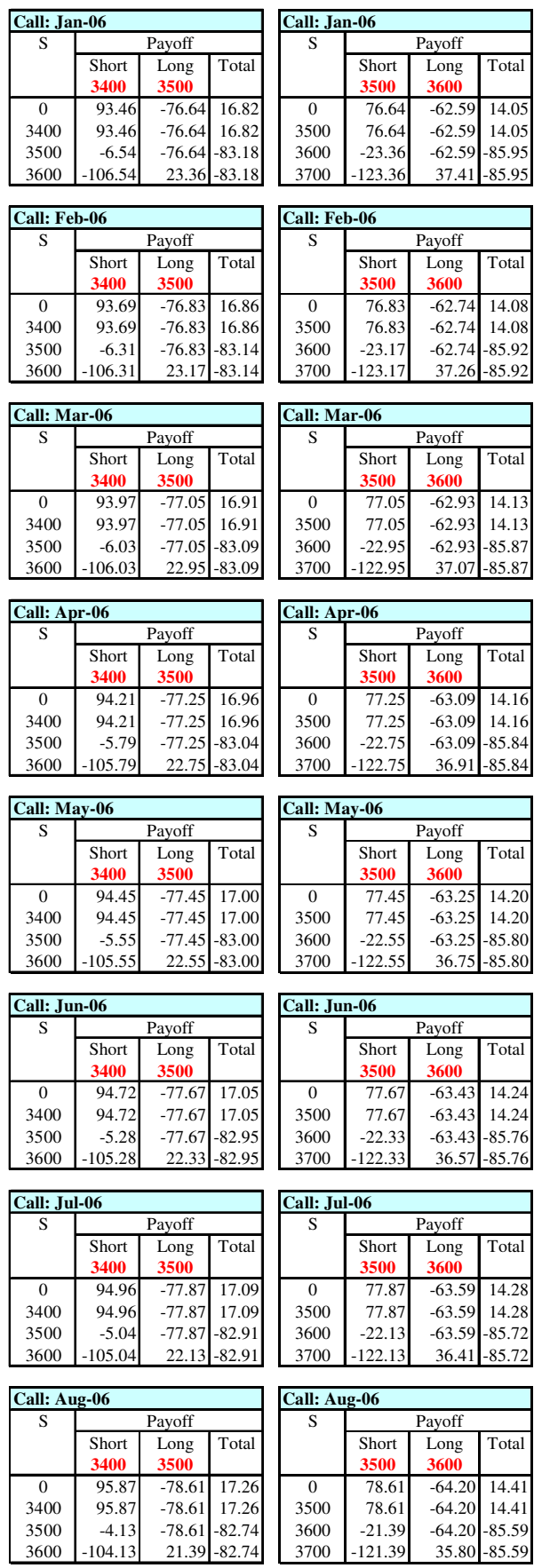
Table 7.16 continued
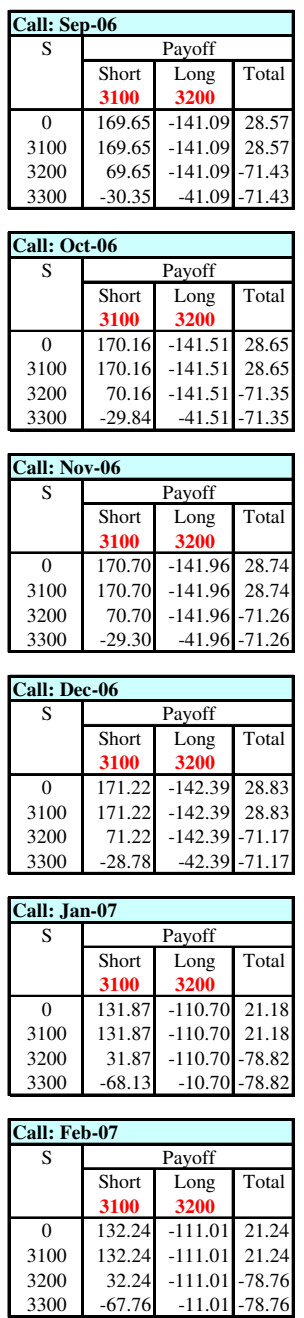

\begin{tabular}{|c|c|c|c|}
\hline \multicolumn{4}{|c|}{ Call: Mar-07 } \\
\hline \multirow[t]{2}{*}{$\mathrm{S}$} & \multicolumn{3}{|c|}{ Payoff } \\
\hline & $\begin{array}{l}\text { Short } \\
3100\end{array}$ & $\begin{array}{l}\text { Long } \\
3200\end{array}$ & Total \\
\hline 0 & 132.67 & -111.36 & 21.30 \\
\hline 3100 & 132.67 & -111.36 & 21.30 \\
\hline 3200 & 32.67 & -111.36 & -78.70 \\
\hline 3300 & -67.33 & -11.36 & -78.70 \\
\hline
\end{tabular}

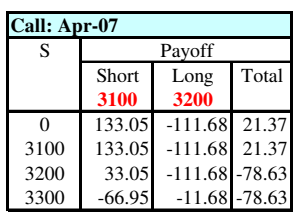

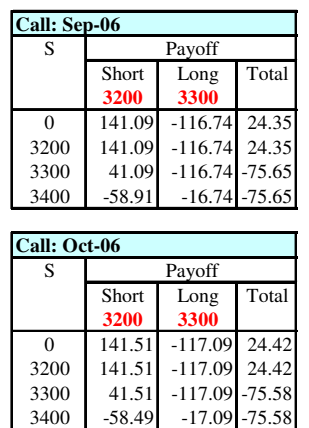
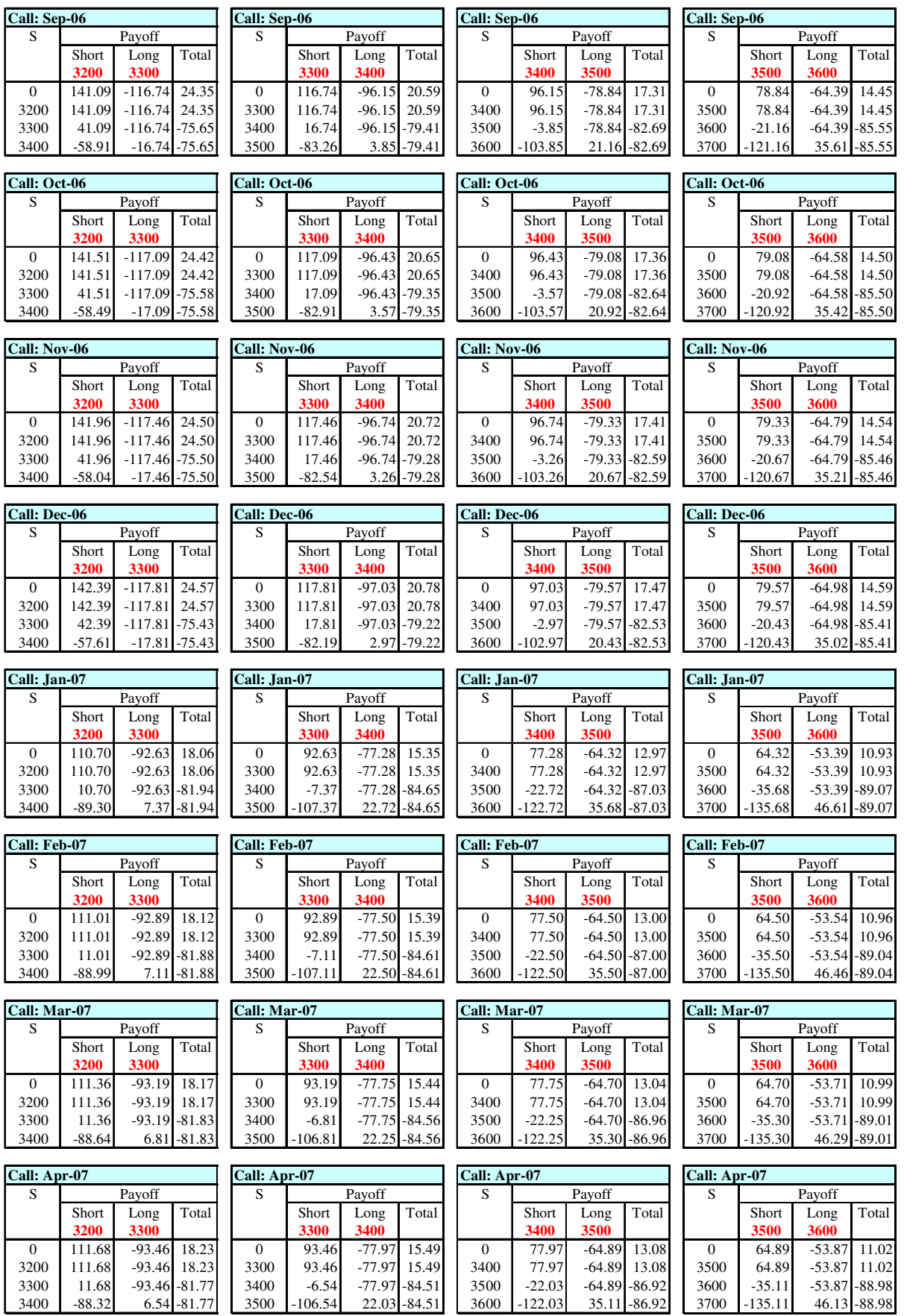
Table 7.16 continued
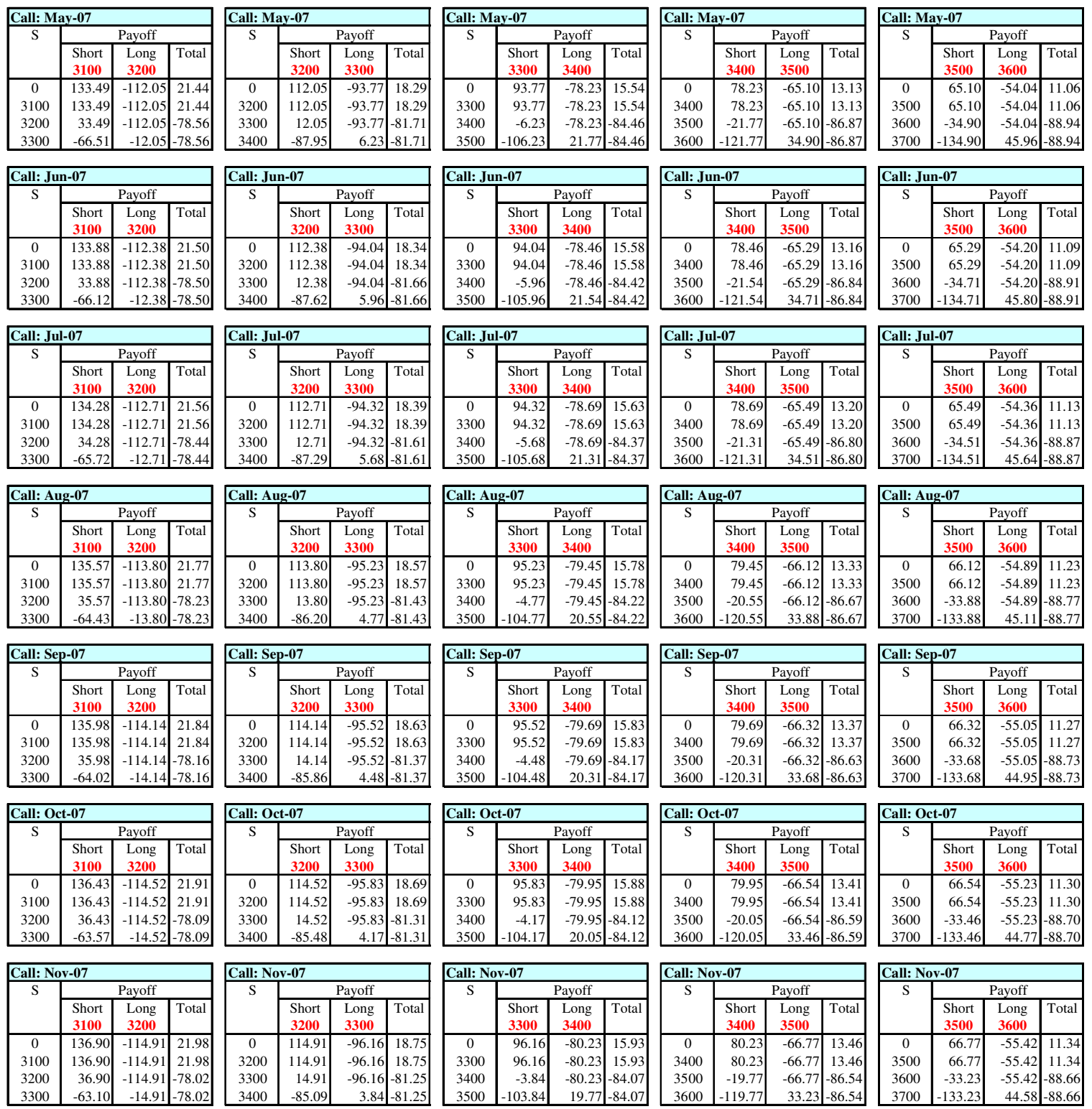

\begin{tabular}{|c|r|r|r|}
\hline \multicolumn{4}{|c|}{ Call: Nov-07 } \\
\hline $\mathrm{S}$ & \multicolumn{3}{|c|}{ Payoff } \\
\cline { 2 - 4 } & \multicolumn{1}{|c|}{ Short } & \multicolumn{1}{|c|}{ Long } & Total \\
& \multicolumn{1}{|c|}{$\mathbf{3 2 0 0}$} & $\mathbf{3 3 0 0}$ & \\
\hline 0 & 114.91 & -96.16 & 18.75 \\
3200 & 114.91 & -96.16 & 18.75 \\
3300 & 14.91 & -96.16 & -81.25 \\
3400 & -85.09 & 3.84 & -81.25 \\
\hline
\end{tabular}

\begin{tabular}{|c|r|r|r|}
\hline \multicolumn{4}{|c|}{ Call: Nov-07 } \\
\hline \multirow{2}{*}{$\mathrm{S}$} & \multicolumn{3}{|c|}{ Payoff } \\
\cline { 2 - 4 } & Short & Long & Total \\
& $\mathbf{3 3 0 0}$ & $\mathbf{3 4 0 0}$ & \\
\hline 0 & 96.16 & -80.23 & 15.93 \\
3300 & 96.16 & -80.23 & 15.93 \\
3400 & -3.84 & -80.23 & -84.07 \\
3500 & -103.84 & 19.77 & -84.07 \\
\hline
\end{tabular}

\begin{tabular}{|c|r|r|r|}
\hline \multicolumn{4}{|c|}{ Call: Nov-07 } \\
\hline \multirow{2}{*}{$S$} & \multicolumn{3}{|c|}{ Payoff } \\
\cline { 2 - 4 } & Short & Long & Total \\
& $\mathbf{3 4 0 0}$ & $\mathbf{3 5 0 0}$ & \\
\hline 0 & 80.23 & -66.77 & 13.46 \\
3400 & 80.23 & -66.77 & 13.46 \\
3500 & -19.77 & -66.77 & -86.54 \\
3600 & -119.77 & 33.23 & -86.54 \\
\hline
\end{tabular}
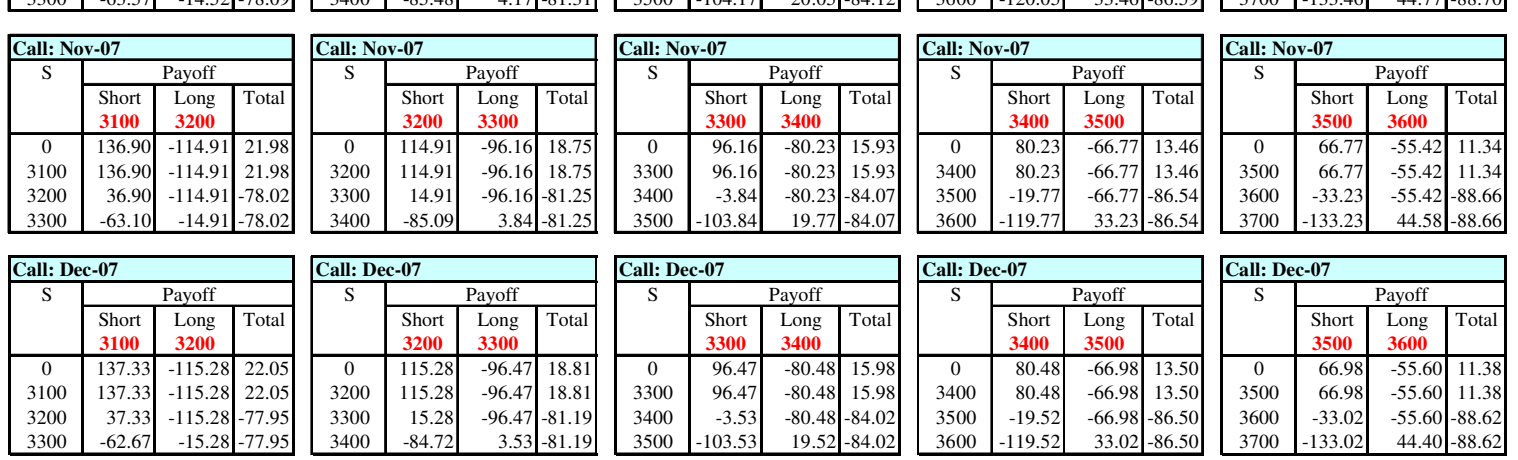


\section{Bear Spreads Using Put Options:}

Bear spreads can also be created by put options. Nevertheless, monthly average copper prices will likely be more than the maximum strike price $(\$ 2,700)$ of all put options, quoted on 22 February 2005 date used here, so creating a bear spread using put options is not optimal than using a call options. This is demonstrated by the table below.

Table 7.17: Bear Spreads Using Put Options

\begin{tabular}{|c|r|c|r|r|r|r|r|}
\hline \multicolumn{4}{|c|}{ Put: Dec-06 } & \multicolumn{4}{|c|}{ Put: Dec-07 } \\
\hline \multirow{3}{*}{ S } & \multicolumn{3}{|c|}{ Payoff } & \multirow{2}{*}{ S } & \multicolumn{3}{|c|}{ Payoff } \\
\cline { 2 - 4 } \cline { 6 - 8 } & Short & Long & Total & & Short & Long & Total \\
& $\mathbf{2 6 0 0}$ & $\mathbf{2 7 0 0}$ & & & $\mathbf{2 6 0 0}$ & $\mathbf{2 7 0 0}$ & \\
\hline 0 & -2425.82 & 2480.17 & 54.35 & 0 & -2320.07 & 2362.25 & 42.19 \\
2600 & 174.18 & -119.83 & 54.35 & 2600 & 279.93 & -237.75 & 42.19 \\
2700 & 174.18 & -219.83 & -45.65 & 2700 & 279.93 & -337.75 & -57.81 \\
2800 & 174.18 & -219.83 & -45.65 & 2800 & 279.93 & -337.75 & -57.81 \\
\hline
\end{tabular}

In table (7.17), there are two bear spreads, created using put options for Dec-06 and Dec-07. The total payoff for these spreads will be negative if monthly average copper prices for Dec-06 and Dec-07 are $\$ 2,700$ or more. The probability for monthly average copper prices to be less than $\$ 2,700$ in 2006 and 2007 is zero, according to the simulation results in chapter 6; therefore, bear spreads using put options would not be profitable.

If estimated forecasts using simulation analysis in chapter 6 prove to be wrong, the opposite spread types can be used. For instance, instead of using a bear spread strategy that was created by taking a long call option position at $\$ 3,500$ and a short call option position at $\$ 3,400$, a bull spread that was created by taking a long call option position at $\$ 3,400$ and a short call option position at $\$ 3,500$ would be useful if the average price was more likely to settle around $\$ 3,500$ or over. Therefore, if a bull spread for a specific position is profitable, a bear spread for that position is not profitable and vice versa. 


\subsubsection{BUTTERFLY SPREADS:}

A butterfly spread is a common and useful strategy involving options. It can be used in the situation where the underlying price is unlikely to fluctuate significantly or where it is expected to move significantly from the middle strike price in either direction. Generally, the middle strike price is close to the current asset price. A butterfly spread is profitable if the asset price is settled close to the middle strike price. Otherwise, there will be a small loss. This strategy involves option positions with three different exercise prices. Both call and put options can be used for creating a butterfly spread. If options with the lowest and highest strike prices expected are bought, then two options with strike prices, halfway between the highest and lowest strikes are sold. For example, on 22 February 2005 , an option trader could buy call options at $\$ 3,400$ and $\$ 3,600$ and write two call options at $\$ 3,500$ if the option trader anticipated that monthly average copper price was likely around $\$ 3,500 / t$ for the expiration month. In this case, the option trader could make a profit if the average copper price was settled around $\$ 3,500 / \mathrm{t}$, but the option trader could lose if the average copper price moved significantly away from $\$ 3,500 / \mathrm{t}$.

If two options with the middle strike are bought instead of sold and options with the lowest and highest strike prices are sold, the strategy will reversed. This means that the strategy will result in a gain if there is a significant movement in the underlying price. For instance, on 22 February 2005, an option trader could sell call options at $\$ 3,400$ and $\$ 3,600$ and buy two call options at $\$ 3,500$ if the option trader anticipated that monthly average copper price was likely to move significantly from the middle strike price of $\$ 3,500$. 


\section{Case A: If an investor feels it is unlikely that monthly average cash settlement prices of copper on the LME will move significantly in the future.}

\section{Butterfly Spreads Using Call Options:}

Table (7.26) constructs all possible butterfly spreads using call options for 2006 and 2007. Each spread, two call options were written (sold) at the middle strike price and call options with the lowest and highest strike prices were bought. There were four different butterfly spreads built using call options, so 96 butterfly spreads were available to be used on the date of data. The tables below construct the most profitable four butterfly spread strategies each year in four different cases including monthly average copper price would likely to settle at each possible middle strike prices of $\$ 3,200, \$ 3,300, \$ 3,400$ and $\$ 3,500$.

\section{Butterfly Spread for January 2006 (maximum profit)}

- Spot Price (22 Feb-05):

- Asian Call Options:

- Positions:

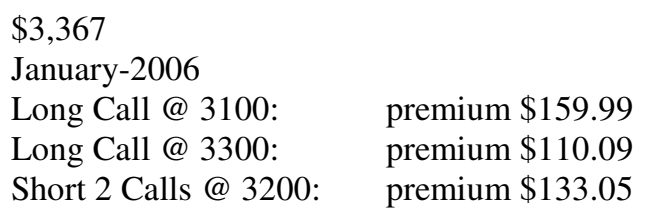

Table 7.18: Profit from a Butterfly Spread (Jan-06 Call options, X: 3100/3200/3300)

\begin{tabular}{ccccc}
\hline $\begin{array}{c}\text { Copper price } \\
\text { Range }\end{array}$ & $\begin{array}{c}\text { Profit from } 1^{\text {st }} \\
\text { long call } \\
\text { @ } 3100\end{array}$ & $\begin{array}{c}\text { Profit from } 2^{\text {nd }} \\
\text { long call } \\
\text { @ 3 3300 }\end{array}$ & $\begin{array}{c}\text { Profit from 2 } \\
\text { short calls } \\
\text { @ 3200 }\end{array}$ & $\begin{array}{c}\text { Total } \\
\text { profit }\end{array}$ \\
\hline $3100>\mathrm{S}_{\mathrm{AVG}}$ & -164.92 & -113.48 & 274.30 & -4.10 \\
$3200>\mathrm{S}_{\mathrm{AVG}}>3100$ & $\mathrm{~S}_{\mathrm{AVG}}-3264.92$ & -113.48 & 274.30 & $\mathrm{~S}_{\mathrm{AVG}}-3104.10$ \\
$3300>\mathrm{S}_{\mathrm{AVG}}>3200$ & $\mathrm{~S}_{\mathrm{AVG}}-3264.92$ & -113.48 & $6674.30-2 \mathrm{~S}_{\mathrm{AVG}}$ & $3295.90-\mathrm{S}_{\mathrm{AVG}}$ \\
$\mathrm{S}_{\mathrm{AVG}}>3300$ & $\mathrm{~S}_{\mathrm{AVG}}-3264.92$ & $\mathrm{~S}_{\mathrm{AVG}}-3413.48$ & $6674.30-2 \mathrm{~S}_{\mathrm{AVG}}$ & -4.10 \\
\hline
\end{tabular}

If monthly average price of copper is less than the lowest strike price $(\$ 3,100)$, both bought and written call options would not be exercised and this would result a loss. The total profit would equal the sum of future values of call options' premiums. 
If the average price is between the lowest strike price $(\$ 3,100)$ and the middle strike price $(\$ 3,200)$, the call option that was bought at $\$ 3,100$ would only be exercised and other options would not be exercised. In this case, the payoff from the first long call position at $\$ 3,100$ would be $\left\{-164.92+S_{A V G}-3100\right\}$ and equals $\left\{S_{A V G}-3264.92\right\}$, in which 164.92 is the future value of the call option premium. Both payoffs from the second long call option position and two short call option positions would equal the future values of call options' premiums respectively. Finally, the total payoff would be $\left\{\mathrm{S}_{\mathrm{AVG}}-3104.10\right\}$

If the average price is between the middle strike price $(\$ 3,200)$ and the highest strike price $(\$ 3,300)$, the first long call option would be exercised and its payoff would be the same as the previous. The second long call option would not be exercised, so the payoff from this position would be negative 113.48 , which is the future value of the call option premium. Two call options that were written would be exercised and the payoff from these positions would be $\left\{274.30-2 \mathrm{~S}_{\mathrm{AVG}}+6400\right\}$ and equals $\left\{6674.30-2 \mathrm{~S}_{\mathrm{AVG}}\right\}$. Finally, the total profit would be $\left\{3295.90-S_{\mathrm{AVG}}\right\}$ in this case. If the average price is greater than the highest strike price $(\$ 3,300)$, all call options would be exercised and finally this would result in a loss of $\$ 4.10$.

Profit is made when $\mathrm{S}_{\mathrm{AVG}(\mathrm{Jan}-2006)}$ is in the range $\$ 3,295.90-\$ 3,104.10$ and is maximum of $\$ 95.90$ when $S_{\mathrm{AVG}(\operatorname{Jan}-2006)}$ is $\$ 3,200$.

\section{Butterfly Spread for January 2006 (maximum profit)}

- Spot Price (22 Feb-05):

- Asian Call Options:

- Positions:
$\$ 3,367$

January-2006

Long Call @ 3200: $\quad$ premium $\$ 133.05$

Long Call@ 3400: premium \$90.67

Short 2 Calls @ 3300: premium $\$ 110.09$ 
Table 7.19: Profit from a Butterfly Spread (Jan-06 Call options, X: 3200/3300/3400)

\begin{tabular}{ccccc}
\hline $\begin{array}{c}\text { Copper price } \\
\text { Range }\end{array}$ & $\begin{array}{c}\text { Profit from } 1^{\text {st }} \\
\text { long call } \\
@ 3200\end{array}$ & $\begin{array}{c}\text { Profit from } 2^{\text {nd }} \\
\text { long call } \\
\text { @ 3 3400 }\end{array}$ & $\begin{array}{c}\text { Profit from 2 } \\
\text { short calls } \\
\text { @ 3300 }\end{array}$ & $\begin{array}{c}\text { Total } \\
\text { profit }\end{array}$ \\
\hline $3200>\mathrm{S}_{\mathrm{AVG}}$ & -137.15 & -93.46 & 226.96 & -3.65 \\
$3300>\mathrm{S}_{\mathrm{AVG}}>3200$ & $\mathrm{~S}_{\mathrm{AVG}}-3337.15$ & -93.46 & 226.96 & $\mathrm{~S}_{\mathrm{AVG}}-3203.65$ \\
$3400>\mathrm{S}_{\mathrm{AVG}}>3300$ & $\mathrm{~S}_{\mathrm{AVG}}-3337.15$ & -93.46 & $6826.96-2 \mathrm{~S}_{\mathrm{AVG}}$ & $3396.35-\mathrm{S}_{\mathrm{AVG}}$ \\
$\mathrm{S}_{\mathrm{AVG}}>3400$ & $\mathrm{~S}_{\mathrm{AVG}}-3337.15$ & $\mathrm{~S}_{\mathrm{AVG}}-3493.46$ & $6826.96-2 \mathrm{~S}_{\mathrm{AVG}}$ & -3.65 \\
\hline
\end{tabular}

Profit is made when $S_{A V G(J a n-2006)}$ is in the range $\$ 3,396.35-\$ 3,203.65$ and is maximum of $\$ 96.35$ when $\mathrm{S}_{\mathrm{AVG}(\mathrm{Jan}-2006)}$ is $\$ 3,300$.

Butterfly Spread for January 2006 (maximum profit)

- Spot Price (22 Feb-05):

- Asian Call Options:

- Positions:
$\$ 3,367$

January-2006

Long Call @ 3300: $\quad$ premium $\$ 110.09$

Long Call@ 3500:_ premium \$74.35

Short 2 Calls@ 3400: premium $\$ 90.67$

Table 7.20: Profit from a Butterfly Spread (Jan-06 Call options, X: 3300/3400/3500)

\begin{tabular}{ccccc}
\hline $\begin{array}{c}\text { Copper price } \\
\text { Range }\end{array}$ & $\begin{array}{c}\text { Profit from } 1^{\text {st }} \\
\text { long call } \\
@ 3300\end{array}$ & $\begin{array}{c}\text { Profit from } 2^{\text {nd }} \\
\text { long call } \\
@ ~ @ 3500\end{array}$ & $\begin{array}{c}\text { Profit from 2 } \\
\text { short calls } \\
@ 3400\end{array}$ & $\begin{array}{c}\text { Total } \\
\text { profit }\end{array}$ \\
\hline $3300>\mathrm{S}_{\mathrm{AVG}}$ & -113.48 & -76.64 & 186.93 & -3.19 \\
$3400>\mathrm{S}_{\mathrm{AVG}}>3300$ & $\mathrm{~S}_{\mathrm{AVG}}-3413.48$ & -76.64 & 186.93 & $\mathrm{~S}_{\mathrm{AVG}}-3303.19$ \\
$3500>\mathrm{S}_{\mathrm{AVG}}>3400$ & $\mathrm{~S}_{\mathrm{AVG}}-3413.48$ & -76.64 & $6986.93-2 \mathrm{~S}_{\mathrm{AVG}}$ & $3496.81-\mathrm{S}_{\mathrm{AVG}}$ \\
$\mathrm{S}_{\mathrm{AVG}}>3500$ & $\mathrm{~S}_{\mathrm{AVG}}-3413.48$ & $\mathrm{~S}_{\mathrm{AVG}}-3576.64$ & $6986.93-2 \mathrm{~S}_{\mathrm{AVG}}$ & -3.19 \\
\hline
\end{tabular}

Profit is made when $\mathrm{S}_{\mathrm{AVG}(\mathrm{Jan}-2006)}$ is in the range $\$ 3,496.81-\$ 3,303.19$ and is maximum of $\$ 96.80$ when $S_{\mathrm{AVG}(\operatorname{Jan}-2006)}$ is $\$ 3,400$.

\section{Butterfly Spread for January 2006 (maximum profit)}

- Spot Price (22 Feb-05):

- Asian Call Options:

- Positions:
$\$ 3,367$

January-2006

Long Call @ 3400: $\quad$ premium $\$ 90.67$

Long Call @ 3600: $\quad$ premium $\$ 60.72$

Short 2 Calls @ 3500: premium \$74.35 
Table 7.21: Profit from a Butterfly Spread (Jan-06 Call options, X: 3400/3500/3600)

\begin{tabular}{ccccc}
\hline $\begin{array}{c}\text { Copper price } \\
\text { Range }\end{array}$ & $\begin{array}{c}\text { Profit from } 1^{\text {st }} \\
\text { long call } \\
@ 3400\end{array}$ & $\begin{array}{c}\text { Profit from } 2^{\text {nd }} \\
\text { long call } \\
\text { @ } 3600\end{array}$ & $\begin{array}{c}\text { Profit from 2 } \\
\text { short calls } \\
\text { @ 3500 }\end{array}$ & $\begin{array}{c}\text { Total } \\
\text { profit }\end{array}$ \\
\hline $3400>\mathrm{S}_{\mathrm{AVG}}$ & -93.46 & -62.59 & 153.28 & -2.77 \\
$3500>\mathrm{S}_{\mathrm{AVG}}>3400$ & $\mathrm{~S}_{\mathrm{AVG}}-3493.46$ & -62.59 & 153.28 & $\mathrm{~S}_{\mathrm{AVG}}-3402.77$ \\
$3600>\mathrm{S}_{\mathrm{AVG}}>3500$ & $\mathrm{~S}_{\mathrm{AVG}}-3493.46$ & -62.59 & $7153.28-2 \mathrm{~S}_{\mathrm{AVG}}$ & $3597.23-\mathrm{S}_{\mathrm{AVG}}$ \\
$\mathrm{S}_{\mathrm{AVG}}>3600$ & $\mathrm{~S}_{\mathrm{AVG}}-3493.46$ & $\mathrm{~S}_{\mathrm{AVG}}-3662.59$ & $7153.28-2 \mathrm{~S}_{\mathrm{AVG}}$ & -2.77 \\
\hline
\end{tabular}

Profit is made when $\mathrm{S}_{\mathrm{AVG}(\mathrm{Jan}-2006)}$ is in the range $\$ 3,597.23-\$ 3,402.77$ and is maximum of $\$ 97.23$ when $\mathrm{S}_{\mathrm{AVG}(\mathrm{Jan}-2006)}$ is $\$ 3,500$.

Figure (7.14): Butterfly Spread, Call Options Jan-06, X: 3100/3200/3300

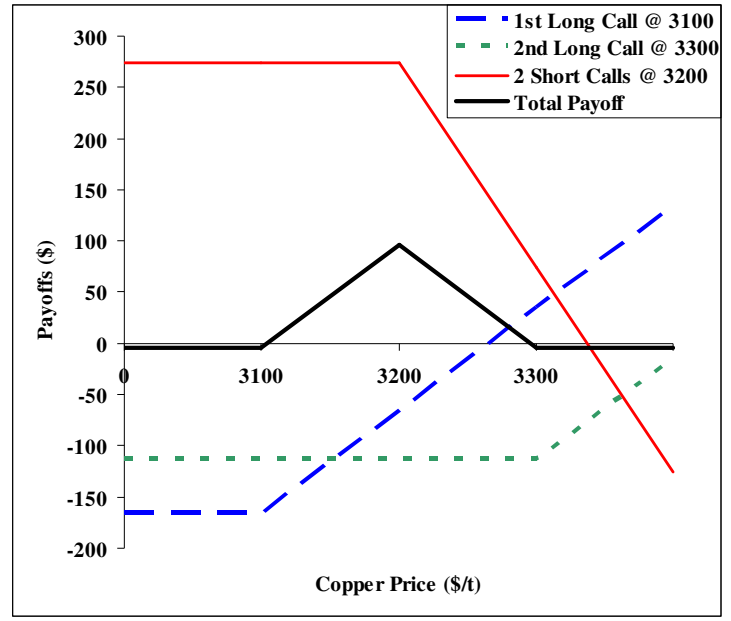

Figure (7.15): Butterfly Spread, Call Options Jan-06, X: 3200/3300/3400

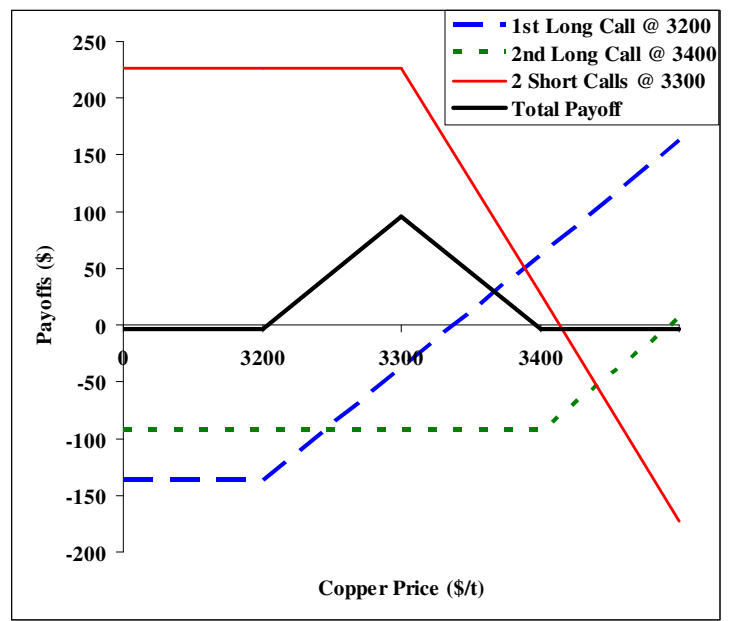

Figure (7.16): Butterfly Spread, Call Options Jan-06, X: 3300/3400/3500

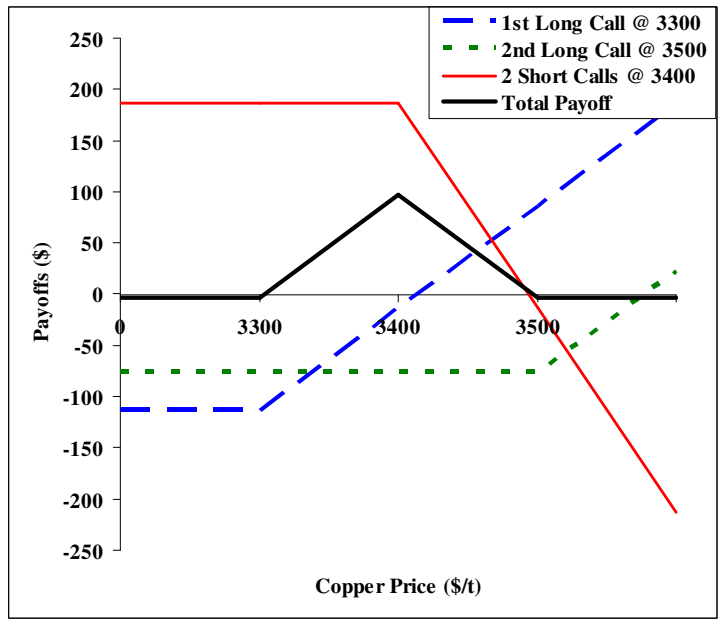

Figure (7.17): Butterfly Spread, Call Options Jan-06, X: 3400/3500/3600

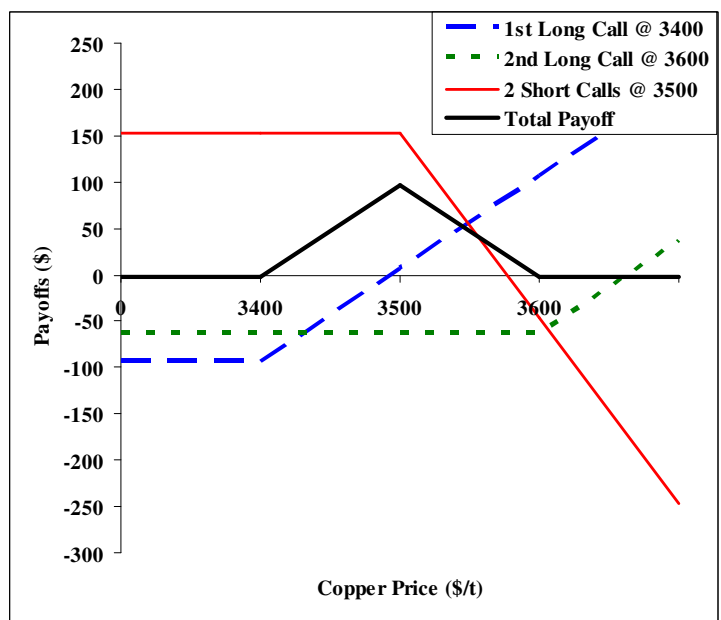


Butterfly Spread for January 2007 (maximum profit)

- Spot Price (22 Feb-05):

$\$ 3,367$

- Asian Call Options:

January-2007

- Positions:

Long Call @ 3100: $\quad$ premium $\$ 122.86$

Long Call @ 3300: $\quad$ premium $\$ 86.30$

Short 2 Calls @ 3200: premium $\$ 103.13$

Table 7.22: Profit from a Butterfly Spread (Jan-07 Call options, X: 3100/3200/3300)

\begin{tabular}{ccccc}
\hline $\begin{array}{c}\text { Copper price } \\
\text { Range }\end{array}$ & $\begin{array}{c}\text { Profit from } 1^{\text {st }} \\
\text { long call } \\
@ 3100\end{array}$ & $\begin{array}{c}\text { Profit from } 2^{\text {nd }} \\
\text { long call } \\
\text { @ 3300 }\end{array}$ & $\begin{array}{c}\text { Profit from 2 } \\
\text { short calls } \\
\text { @ 3200 }\end{array}$ & $\begin{array}{c}\text { Total } \\
\text { profit }\end{array}$ \\
\hline $3100>\mathrm{S}_{\mathrm{AVG}}$ & -131.87 & -92.63 & 221.39 & -3.11 \\
$3200>\mathrm{S}_{\mathrm{AVG}}>3100$ & $\mathrm{~S}_{\mathrm{AVG}}-3231.87$ & -92.63 & 221.39 & $\mathrm{~S}_{\mathrm{AVG}}-3103.11$ \\
$3300>\mathrm{S}_{\mathrm{AVG}}>3200$ & $\mathrm{~S}_{\mathrm{AVG}}-3231.87$ & -92.63 & $6621.39-2 \mathrm{~S}_{\mathrm{AVG}}$ & $3296.89-\mathrm{S}_{\mathrm{AVG}}$ \\
$\mathrm{S}_{\mathrm{AVG}}>3300$ & $\mathrm{~S}_{\mathrm{AVG}}-3231.87$ & $\mathrm{~S}_{\mathrm{AVG}}-3392.63$ & $6621.39-2 \mathrm{~S}_{\mathrm{AVG}}$ & -3.11 \\
\hline
\end{tabular}

Profit is made when $\mathrm{S}_{\mathrm{AVG}(\mathrm{Jan}-2007)}$ is in the range $\$ 3,296.89-\$ 3,103.11$ and is maximum of $\$ 96.89$ when $\mathrm{S}_{\mathrm{AVG}(\operatorname{Jan}-2007)}$ is $\$ 3,200$.

\section{Butterfly Spread for January 2007 (maximum profit)}

- Spot Price (22 Feb-05):

- Asian Call Options:

- Positions:
$\$ 3,367$

January-2007

Long Call @ 3200: $\quad$ premium $\$ 103.13$

Long Call @ 3400: $\quad$ premium $\$ 72.00$

Short 2 Calls @ 3300: premium $\$ 86.30$

Table 7.23: Profit from a Butterfly Spread (Jan-07 Call options, X: 3200/3300/3400)

\begin{tabular}{ccccc}
\hline $\begin{array}{c}\text { Copper price } \\
\text { Range }\end{array}$ & $\begin{array}{c}\text { Profit from } 1^{\text {st }} \\
\text { long call } \\
\text { @ } 3200\end{array}$ & $\begin{array}{c}\text { Profit from } 2^{\text {nd }} \\
\text { long call } \\
\text { @ 3 3400 }\end{array}$ & $\begin{array}{c}\text { Profit from 2 } \\
\text { short calls } \\
\text { @ 3300 }\end{array}$ & $\begin{array}{c}\text { Total } \\
\text { profit }\end{array}$ \\
\hline $3200>\mathrm{S}_{\mathrm{AVG}}$ & -110.70 & -77.28 & 185.26 & -2.72 \\
$3300>\mathrm{S}_{\mathrm{AVG}}>3200$ & $\mathrm{~S}_{\mathrm{AVG}}-3310.70$ & -77.28 & 185.26 & $\mathrm{~S}_{\mathrm{AVG}}-3202.72$ \\
$3400>\mathrm{S}_{\mathrm{AVG}}>3300$ & $\mathrm{~S}_{\mathrm{AVG}}-3310.70$ & -77.28 & $6785.26-2 \mathrm{~S}_{\mathrm{AVG}}$ & $3397.28-\mathrm{S}_{\mathrm{AVG}}$ \\
$\mathrm{S}_{\mathrm{AVG}}>3400$ & $\mathrm{~S}_{\mathrm{AVG}}-3310.70$ & $\mathrm{~S}_{\mathrm{AVG}}-3477.28$ & $6785.26-2 \mathrm{~S}_{\mathrm{AVG}}$ & -2.72 \\
\hline
\end{tabular}

Profit is made when $\mathrm{S}_{\mathrm{AVG}(\mathrm{Jan}-2007)}$ is in the range $\$ 3,397.28-\$ 3,202.72$ and is maximum of $\$ 97.28$ when $\mathrm{S}_{\mathrm{AVG}(\operatorname{Jan}-2007)}$ is $\$ 3,300$. 
Butterfly Spread for January 2007 (maximum profit)

- Spot Price (22 Feb-05):

$\$ 3,367$

- Asian Call Options:

January-2007

- Positions:

Long Call @ 3300: $\quad$ premium $\$ 86.30$

Long Call @ 3500: $\quad$ premium $\$ 59.92$

Short 2 Calls @ 3400: premium $\$ 72.00$

Table 7.24: Profit from a Butterfly Spread (Jan-07 Call options, X: 3300/3400/3500)

\begin{tabular}{ccccc}
\hline $\begin{array}{c}\text { Copper price } \\
\text { Range }\end{array}$ & $\begin{array}{c}\text { Profit from } 1^{\text {st }} \\
\text { long call } \\
\text { @ 3300 }\end{array}$ & $\begin{array}{c}\text { Profit from } 2^{\text {nd }} \\
\text { long call } \\
\text { @ 3 3500 }\end{array}$ & $\begin{array}{c}\text { Profit from 2 } \\
\text { short calls } \\
\text { @ 3400 }\end{array}$ & $\begin{array}{c}\text { Total } \\
\text { profit }\end{array}$ \\
\hline $3300>\mathrm{S}_{\mathrm{AVG}}$ & -92.63 & -64.32 & 154.57 & -2.38 \\
$3400>\mathrm{S}_{\mathrm{AVG}}>3300$ & $\mathrm{~S}_{\mathrm{AVG}}-3392.63$ & -64.32 & 154.57 & $\mathrm{~S}_{\mathrm{AVG}}-3302.38$ \\
$3500>\mathrm{S}_{\mathrm{AVG}}>3400$ & $\mathrm{~S}_{\mathrm{AVG}}-3392.63$ & -64.32 & $6954.57-2 \mathrm{~S}_{\mathrm{AVG}}$ & $3497.62-\mathrm{S}_{\mathrm{AVG}}$ \\
$\mathrm{S}_{\mathrm{AVG}}>3500$ & $\mathrm{~S}_{\mathrm{AVG}}-3392.63$ & $\mathrm{~S}_{\mathrm{AVG}}-3564.32$ & $6954.57-2 \mathrm{~S}_{\mathrm{AVG}}$ & -2.38 \\
\hline
\end{tabular}

Profit is made when $\mathrm{S}_{\mathrm{AVG}(\mathrm{Jan}-2007)}$ is in the range $\$ 3,497.62-\$ 3,302.38$ and is maximum of $\$ 97.62$ when $\mathrm{S}_{\mathrm{AVG}(\operatorname{Jan}-2007)}$ is $\$ 3,400$.

\section{Butterfly Spread for January 2007 (maximum profit)}

- Spot Price (22 Feb-05):

- Asian Call Options:

- Positions:
$\$ 3,367$

January-2007

Long Call @ 3400: $\quad$ premium $\$ 72.00$

Long Call @ 3600: $\quad$ premium $\$ 49.74$

Short 2 Calls @ 3500: premium $\$ 59.92$

Table 7.25: Profit from a Butterfly Spread (Jan-07 Call options, X: 3400/3500/3600)

\begin{tabular}{ccccc}
\hline $\begin{array}{c}\text { Copper price } \\
\text { Range }\end{array}$ & $\begin{array}{c}\text { Profit from } 1^{\text {st }} \\
\text { long call } \\
\text { @ 3400 }\end{array}$ & $\begin{array}{c}\text { Profit from } 2^{\text {nd }} \\
\text { long call } \\
\text { @ 3600 }\end{array}$ & $\begin{array}{c}\text { Profit from 2 } \\
\text { short calls } \\
\text { @ 3500 }\end{array}$ & $\begin{array}{c}\text { Total } \\
\text { profit }\end{array}$ \\
\hline $3400>\mathrm{S}_{\mathrm{AVG}}$ & -77.28 & -53.39 & 128.63 & -2.04 \\
$3500>\mathrm{S}_{\mathrm{AVG}}>3400$ & $\mathrm{~S}_{\mathrm{AVG}}-3477.28$ & -53.39 & 128.63 & $\mathrm{~S}_{\mathrm{AVG}}-3402.04$ \\
$3600>\mathrm{S}_{\mathrm{AVG}}>3500$ & $\mathrm{~S}_{\mathrm{AVG}}-3477.28$ & -53.39 & $7128.63-2 \mathrm{~S}_{\mathrm{AVG}}$ & $3597.96-\mathrm{S}_{\mathrm{AVG}}$ \\
$\mathrm{S}_{\mathrm{AVG}}>3600$ & $\mathrm{~S}_{\mathrm{AVG}}-3477.28$ & $\mathrm{~S}_{\mathrm{AVG}}-3653.39$ & $7128.63-2 \mathrm{~S}_{\mathrm{AVG}}$ & -2.04 \\
\hline
\end{tabular}

Profit is made when $\mathrm{S}_{\mathrm{AVG}(\mathrm{Jan}-2007)}$ is in the range $\$ 3,597.96-\$ 3,402.04$ and is maximum of $\$ 97.96$ when $S_{\mathrm{AVG}(\operatorname{Jan}-2007)}$ is $\$ 3,500$. 
Figure (7.18): Butterfly Spread, Call Options Jan-07, X: 3100/3200/3300

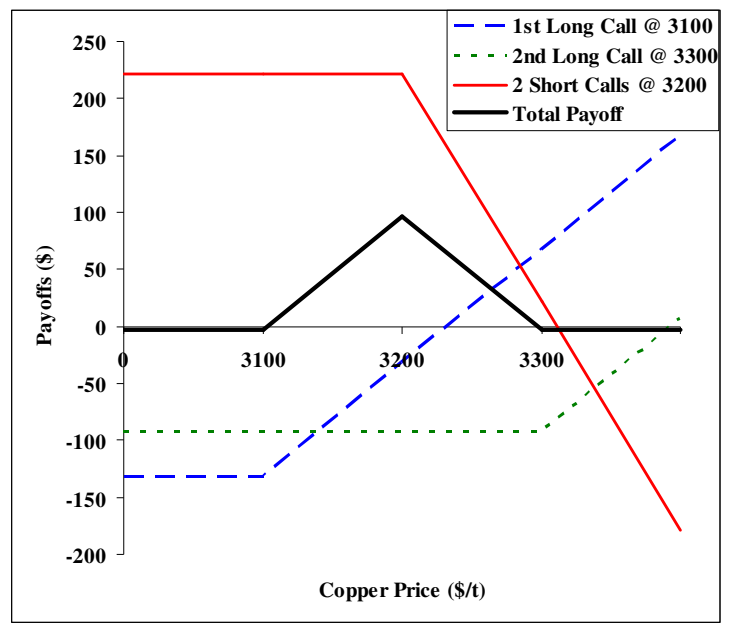

Figure (7.19): Butterfly Spread, Call Options Jan-07, X: 3200/3300/3400

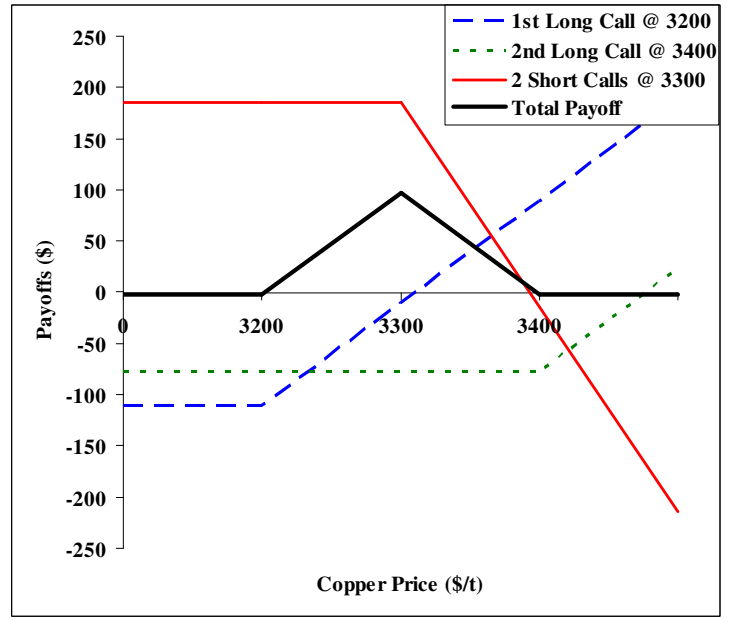

Figure (7.20): Butterfly Spread, Call Options Jan-07, X: 3300/3400/3500

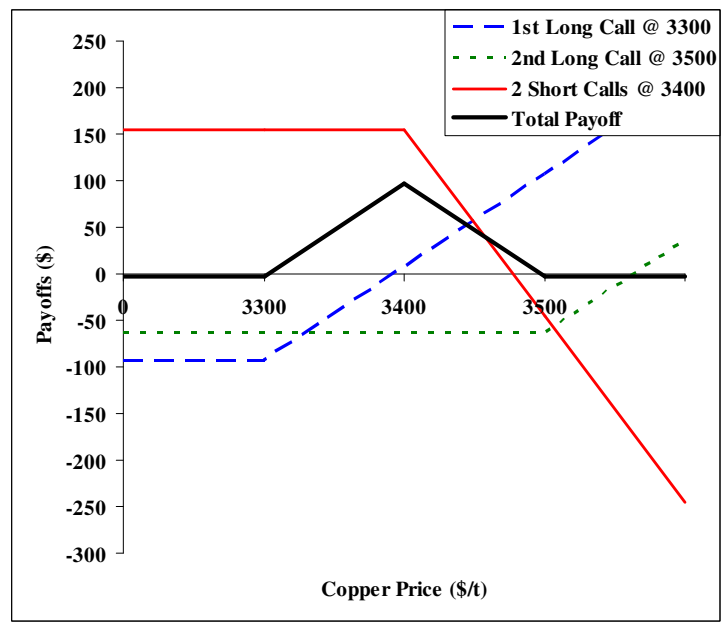

Figure (7.21): Butterfly Spread, Call Options Jan-07, X: 3400/3500/3600

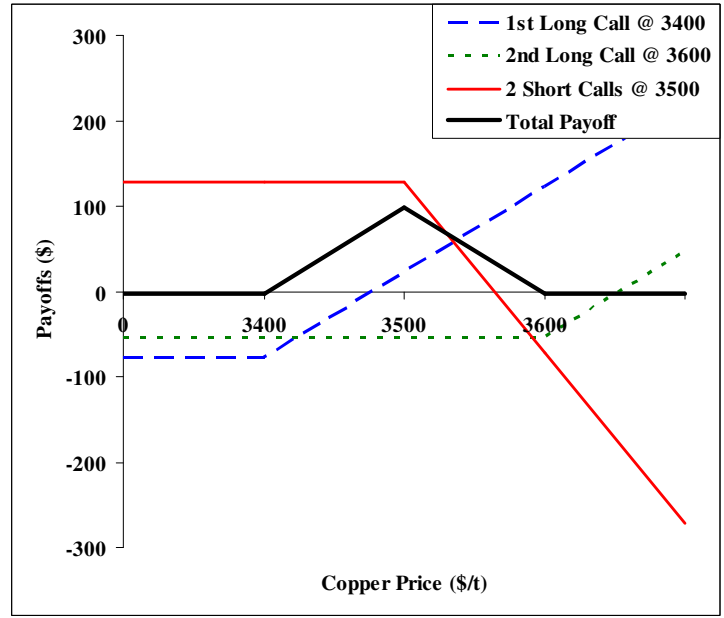

Comparing these 96 butterfly spreads, which were created using call options in 2006 and 2007, the spreads on Jan-06 and Jan-07 calls were more effective because if the price fluctuates in either direction from the middle strike prices $(\$ 3200, \$ 3300, \$ 3400, \$ 3500)$ the loss would be no more than $\$ 4.10, \$ 3.65, \$ 3.19, \$ 2.77$ for January 2006 spreads and \$3.11, \$2.72, \$2.38, \$2.04 for January 2007 spreads respectively. Furthermore, the gains would be greatest if the price settles around the middle strike prices. 
Table 7.26: Butterfly Spreads Using Call Options for 2006 and 2007 (Case A)

\begin{tabular}{|c|r|r|r|r|}
\hline \multicolumn{4}{|c|}{ Call: Jan-06 } \\
\hline \multirow{2}{*}{$\mathrm{S}$} & \multicolumn{4}{|c|}{ Payoff } \\
\cline { 2 - 5 } & \multicolumn{1}{|c|}{$\mathbf{3 1 0 0}$} & \multicolumn{1}{|c|}{$\mathbf{3 3 0 0}$} & \multicolumn{1}{|c|}{$\mathbf{3 2 0 0}$} & \\
\hline 0 & -164.92 & -113.48 & 274.30 & -4.10 \\
3100 & -164.92 & -113.48 & 274.30 & -4.10 \\
3200 & -64.92 & -113.48 & 274.30 & 95.90 \\
3300 & 35.08 & -113.48 & 74.30 & -4.10 \\
3400 & 135.08 & -13.48 & -125.70 & -4.10 \\
\hline
\end{tabular}

\begin{tabular}{|c|r|r|r|r|}
\hline \multicolumn{5}{|c|}{ Call: Jan-06 } \\
\hline $\mathrm{S}$ & \multicolumn{4}{|c|}{ Payoff } \\
\cline { 2 - 5 } & \multicolumn{1}{|c|}{ 1st Long } & 2nd Long & 2 Short & Total \\
& \multicolumn{1}{|c|}{ 200 } & 3400 & $\mathbf{3 3 0 0}$ & \\
\hline 0 & -137.15 & -93.46 & 226.96 & -3.65 \\
3200 & -137.15 & -93.46 & 226.96 & -3.65 \\
3300 & -37.15 & -93.46 & 226.96 & 96.35 \\
3400 & 62.85 & -93.46 & 26.96 & -3.65 \\
3500 & 162.85 & 6.54 & -173.04 & -3.65 \\
\hline
\end{tabular}

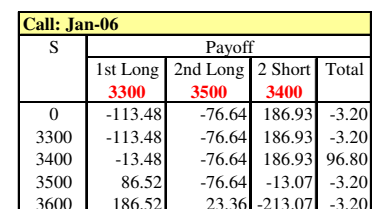

\begin{tabular}{|c|c|c|c|c|}
\hline \multicolumn{5}{|c|}{\begin{tabular}{|l} 
Call: Jan-06 \\
\end{tabular}} \\
\hline \multirow[t]{2}{*}{$\mathrm{S}$} & \multicolumn{4}{|c|}{ Payoff } \\
\hline & $\begin{array}{c}\text { 1st Long } \\
3400\end{array}$ & \begin{tabular}{|c|} 
2nd Long \\
3600
\end{tabular} & \begin{tabular}{|c|}
2 Short \\
3500
\end{tabular} & Total \\
\hline 0 & -93.46 & -62.59 & 153.28 & -2.77 \\
\hline 3400 & -93.46 & -62.59 & 153.28 & -2.77 \\
\hline 3500 & 6.54 & -62.59 & 153.28 & 97.23 \\
\hline 3600 & 106.54 & -62.59 & -46.72 & -2.77 \\
\hline 700 & & & & \\
\hline
\end{tabular}
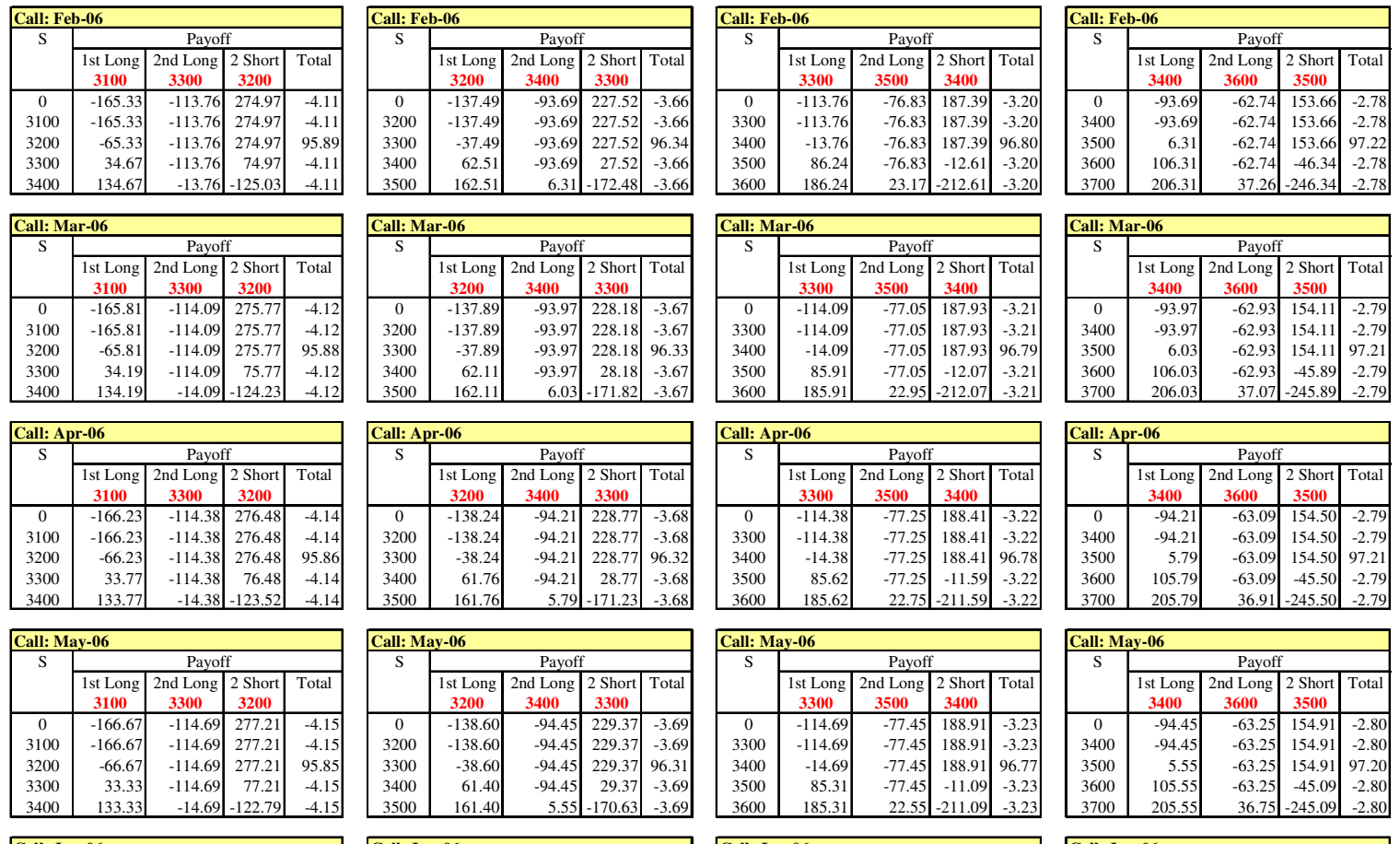

\begin{tabular}{|c|r|c|c|r|}
\hline \multicolumn{5}{|c|}{ Call: Jun-06 } \\
\hline S & \multicolumn{4}{|c|}{ Payoff } \\
\cline { 2 - 5 } & \multicolumn{1}{|c|}{$\mathbf{3 1 0 0}$} & \multicolumn{1}{c|}{$\mathbf{3 3 0 0}$} & 2 Short & Total \\
\hline 0 & -167.14 & -115.01 & 277.99 & -4.16 \\
3100 & -167.14 & -115.01 & 277.99 & -4.16 \\
3200 & -67.14 & -115.01 & 277.99 & 95.84 \\
3300 & 32.86 & -115.01 & 77.99 & -4.16 \\
3400 & 132.86 & -15.01 & -122.01 & -4.16 \\
\hline
\end{tabular}
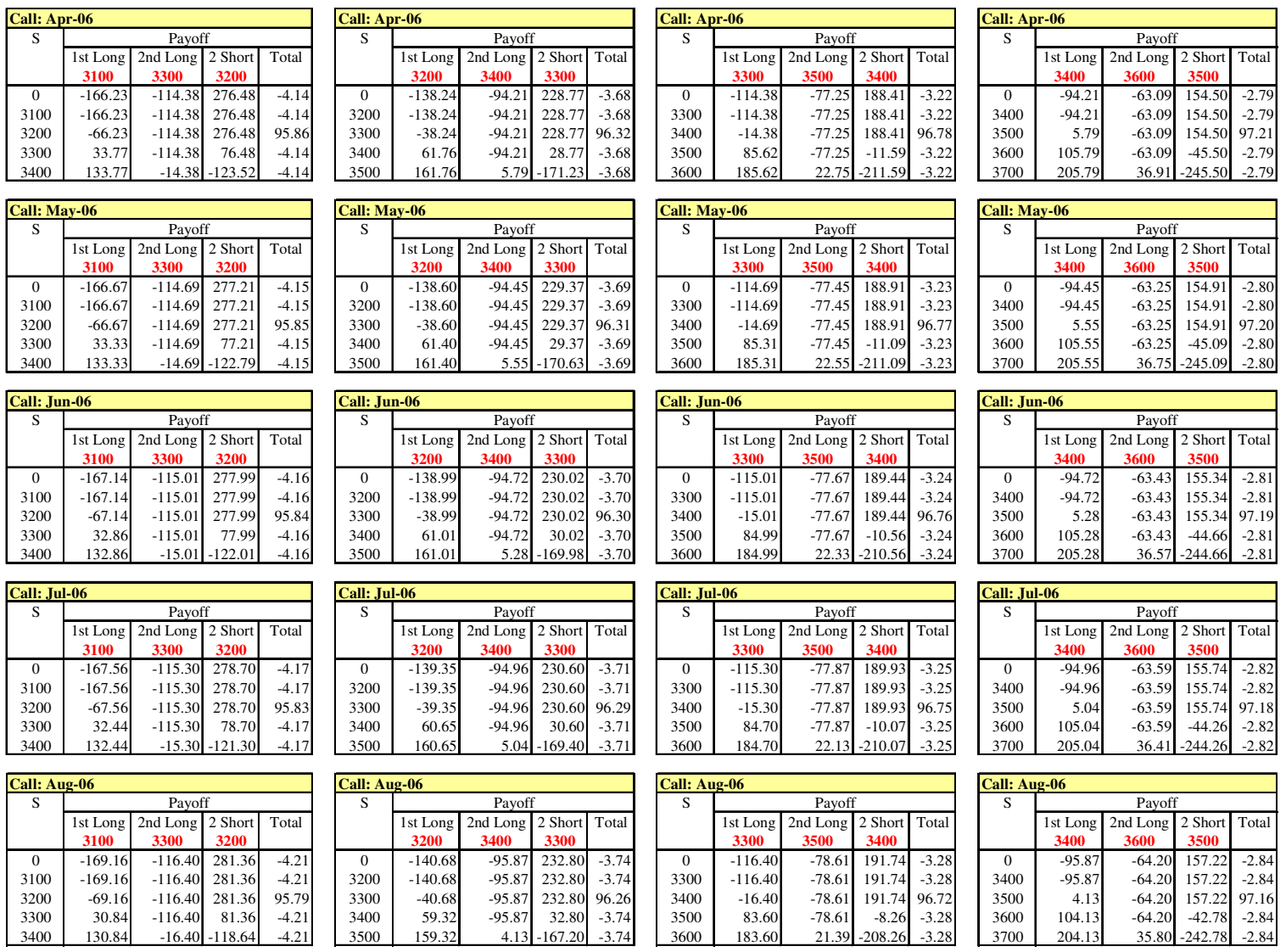

\begin{tabular}{|c|r|c|c|c|}
\hline \multicolumn{5}{|c|}{ Call: Aug-06 } \\
\hline $\mathrm{S}$ & \multicolumn{4}{|c|}{ Payoff } \\
\cline { 2 - 5 } & \multicolumn{1}{c|}{ 1st Long } & 2nd Long & 2 Short & Total \\
\hline 0 & -95.87 & -600 & $\mathbf{3 5 0 0}$ & \\
\hline 3400 & -95.87 & -64.20 & 157.22 & -2.84 \\
3500 & 4.13 & -64.20 & 157.22 & -2.84 \\
3600 & 104.13 & -64.20 & -42.78 & -2.84 \\
3700 & 204.13 & 35.80 & -242.78 & -2.84 \\
\hline
\end{tabular}


Table 7.26 continued

\begin{tabular}{|c|r|r|r|r|}
\hline Call: Sep-06 \\
\hline S & \multicolumn{4}{|c|}{ Payoff } \\
\cline { 2 - 5 } & 1st Long & 2nd Long & 2 Short & Total \\
& \multicolumn{1}{|c|}{$\mathbf{3 1 0 0}$} & \multicolumn{1}{|c|}{$\mathbf{3 3 0 0}$} & \multicolumn{1}{|c|}{$\mathbf{3 2 0 0}$} & \\
\hline 0 & -169.65 & -116.74 & 282.17 & -4.22 \\
3100 & -169.65 & -116.74 & 282.17 & -4.22 \\
3200 & -69.65 & -116.74 & 282.17 & 95.78 \\
3300 & 30.35 & -116.74 & 82.17 & -4.22 \\
3400 & 130.35 & -16.74 & -117.83 & -4.22 \\
\hline
\end{tabular}

\begin{tabular}{|c|c|c|c|c|}
\hline \multicolumn{5}{|c|}{ Call: Oct-06 } \\
\hline \multirow[t]{2}{*}{$\mathrm{S}$} & \multicolumn{4}{|c|}{ Payoff } \\
\hline & \begin{tabular}{c|} 
1st Long \\
3100
\end{tabular} & $\begin{array}{c}\text { 2nd Long } \\
3300\end{array}$ & $\begin{array}{c}2 \text { Short } \\
3200 \\
\end{array}$ & Total \\
\hline 0 & -170.16 & -117.09 & 283.02 & -4.23 \\
\hline 3100 & -170.16 & -117.09 & 283.02 & -4.23 \\
\hline 3200 & -70.16 & -117.09 & 283.02 & 95.77 \\
\hline 3300 & 29.84 & -117.09 & 83.02 & -4.23 \\
\hline 3400 & 129.8 & -17.09 & -116.98 & -4.23 \\
\hline
\end{tabular}

\begin{tabular}{|c|c|c|c|c|}
\hline \multicolumn{5}{|c|}{\begin{tabular}{|l|} 
Call: Nov-06 \\
\end{tabular}} \\
\hline \multirow[t]{2}{*}{ S } & \multicolumn{4}{|c|}{ Payoff } \\
\hline & $\begin{array}{c}\text { 1st Long } \\
3100\end{array}$ & \begin{tabular}{c|} 
2nd Long \\
3300
\end{tabular} & $\begin{array}{c}2 \text { Short } \\
3200\end{array}$ & Total \\
\hline 0 & -170.70 & -117.46 & \begin{tabular}{|l|}
283.92 \\
\end{tabular} & -4.25 \\
\hline 3100 & -170.70 & -117.46 & 283.92 & -4.25 \\
\hline 3200 & -70.70 & -117.46 & 283.92 & 95.75 \\
\hline 3300 & 29.30 & -117.46 & 83.92 & -4.25 \\
\hline 400 & 129.30 & & & \\
\hline
\end{tabular}
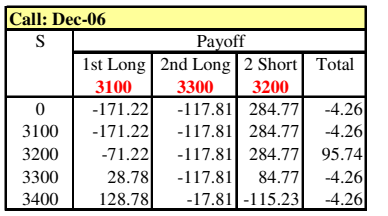

\begin{tabular}{|c|r|r|r|r|}
\hline \multicolumn{5}{|l|}{ Call: Jan-07 } \\
\hline \multirow{2}{*}{$\mathrm{S}$} & \multicolumn{4}{|c|}{ Payoff } \\
\cline { 2 - 5 } & \multicolumn{1}{c|}{$\begin{array}{c}\text { 1st Long } \\
\text { 3100 }\end{array}$} & $\begin{array}{c}\text { 2nd Long } \\
\mathbf{3 3 0 0}\end{array}$ & $\mathbf{3 2 0 0}$ & Total \\
\hline 0 & -131.87 & -92.63 & 221.39 & -3.11 \\
3100 & -131.87 & -92.63 & 221.39 & -3.11 \\
3200 & -31.87 & -92.63 & 221.39 & 96.89 \\
3300 & 68.13 & -92.63 & 21.39 & -3.11 \\
3400 & 168.13 & 7.37 & -178.61 & -3.11 \\
\hline
\end{tabular}

\begin{tabular}{|c|r|r|r|r|}
\hline \multicolumn{5}{|l|}{ Call: Feb-07 } \\
\hline \multirow{2}{*}{$\mathrm{S}$} & \multicolumn{4}{|c|}{ Payoff } \\
\cline { 2 - 5 } & \multicolumn{1}{c|}{$\mathbf{3 1 0 0}$} & \multicolumn{1}{c|}{$\mathbf{3 3 0 0}$} & \multicolumn{3}{|c|}{$\mathbf{3 2 0 0}$} & \\
\hline 0 & -132.24 & -92.89 & 222.01 & -3.12 \\
3100 & -132.24 & -92.89 & 222.01 & -3.12 \\
3200 & -32.24 & -92.89 & 222.01 & 96.88 \\
3300 & 67.76 & -92.89 & 22.01 & -3.12 \\
3400 & 167.76 & 7.11 & -177.99 & -3.12 \\
\hline
\end{tabular}

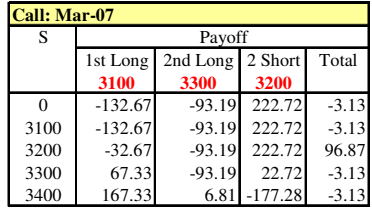

\begin{tabular}{|c|r|r|r|r|}
\hline \multicolumn{4}{|l|}{ Call: Apr-07 } \\
\hline \multirow{2}{*}{$\mathrm{S}$} & \multicolumn{4}{|c|}{ Payoff } \\
\cline { 2 - 5 } & \multicolumn{1}{c|}{$\mathbf{3 1 0 0}$} & \multicolumn{1}{|c|}{$\mathbf{3 3 0 0}$} & $\mathbf{3 2 0 0}$ & \\
\hline 0 & -133.05 & -93.46 & 223.37 & -3.14 \\
3100 & -133.05 & -93.46 & 223.37 & -3.14 \\
3200 & -33.05 & -93.46 & 223.37 & 96.86 \\
3300 & 66.95 & -93.46 & 23.37 & -3.14 \\
3400 & 166.95 & 6.54 & -176.63 & -3.14 \\
\hline
\end{tabular}
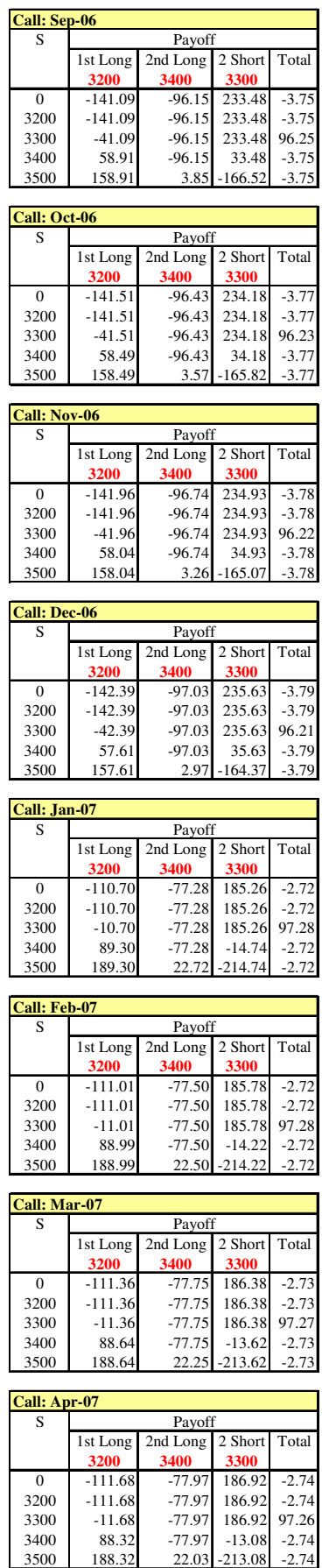
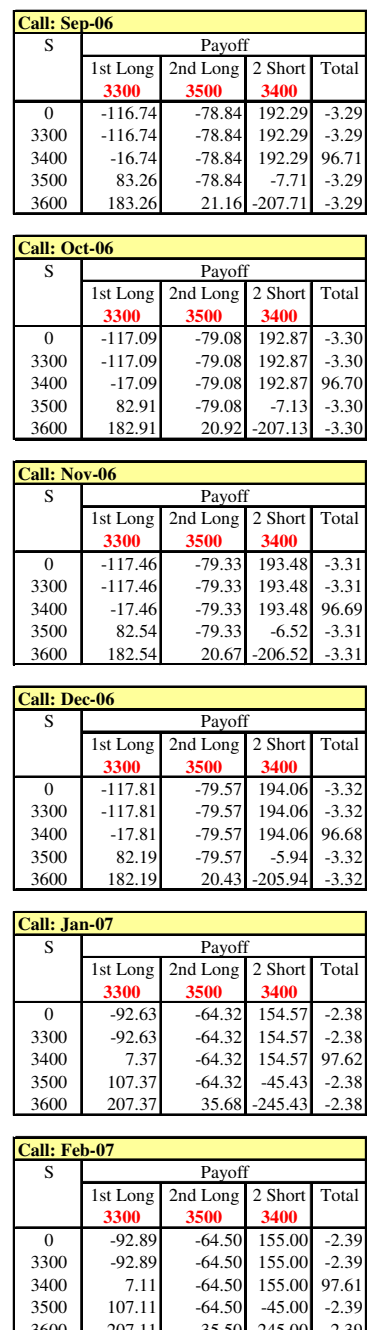

\begin{tabular}{l|l|l|l|l|l|}
3600 & 207.11 & 35.50 & -245.00 & -2.39 \\
\hline
\end{tabular}
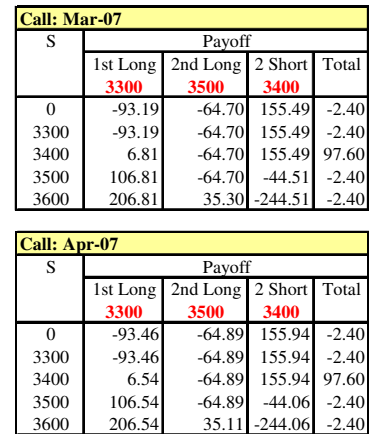
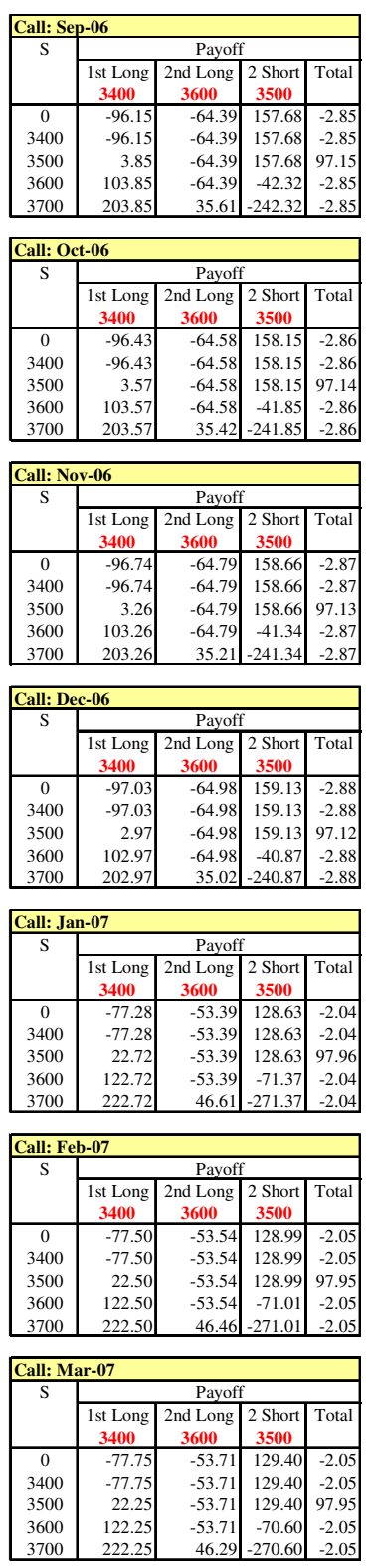

\begin{tabular}{|c|c|c|c|c|}
\hline \multicolumn{5}{|c|}{ Call: Apr-07 } \\
\hline \multirow[t]{2}{*}{$\mathrm{S}$} & \multicolumn{4}{|c|}{ Payoff } \\
\hline & $\begin{array}{c}\text { st Long } \\
3400\end{array}$ & $\begin{array}{c}\text { 2nd Long } \\
3600\end{array}$ & \begin{tabular}{|c|}
2 Short \\
3500
\end{tabular} & Total \\
\hline 0 & -77.97 & -53.87 & 129.78 & -2.06 \\
\hline 3400 & -77.97 & -53.87 & 129.78 & -2.06 \\
\hline 3500 & 22.03 & -53.87 & 129.78 & 97.94 \\
\hline 3600 & 122.03 & -53.87 & -70.22 & -2.06 \\
\hline 3700 & 222.03 & 46.13 & -270.22 & -2.06 \\
\hline
\end{tabular}


Table 7.26 continued
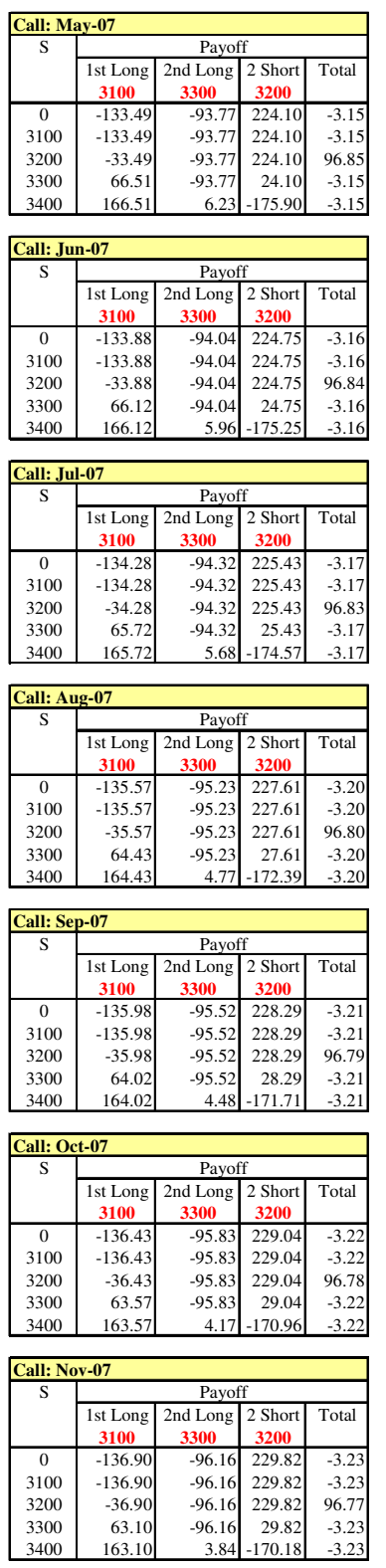

\begin{tabular}{|c|c|c|c|c|}
\hline \multicolumn{5}{|c|}{ Call: Dec-07 } \\
\hline \multirow[t]{2}{*}{$\mathrm{S}$} & \multicolumn{4}{|c|}{ Payoff } \\
\hline & $\begin{array}{c}\text { 1st Long } \\
3100\end{array}$ & $\begin{array}{c}\text { 2nd Long } \\
3300\end{array}$ & \begin{tabular}{|c|}
2 Short \\
3200
\end{tabular} & Total \\
\hline 0 & -137.33 & $\begin{array}{l}-96.47 \\
\end{array}$ & 230.56 & -3.24 \\
\hline 3100 & -137.33 & -96.47 & 230.56 & -3.24 \\
\hline 3200 & -37.33 & -96.47 & 230.56 & 96.76 \\
\hline 3300 & 62.67 & -96.47 & 30.56 & -3.24 \\
\hline U & 162. & e & 169 & -3. \\
\hline
\end{tabular}
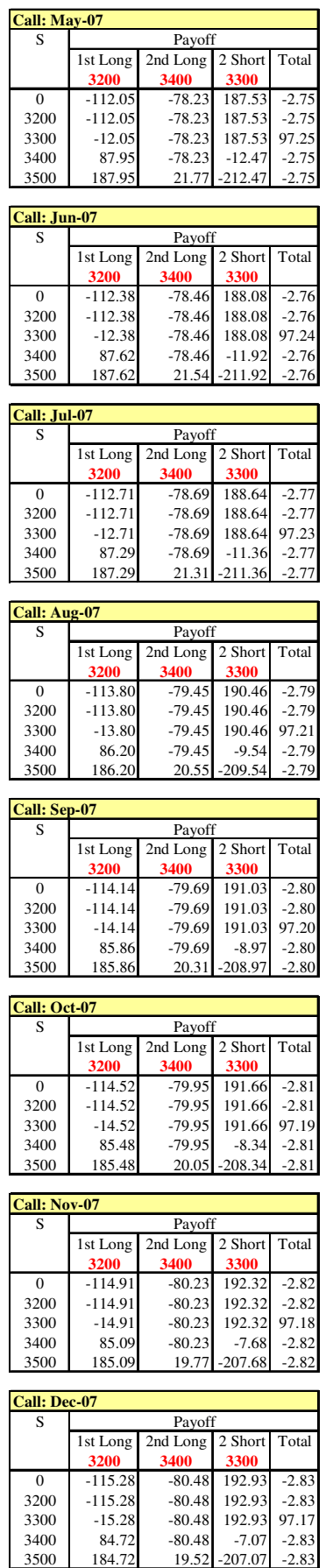
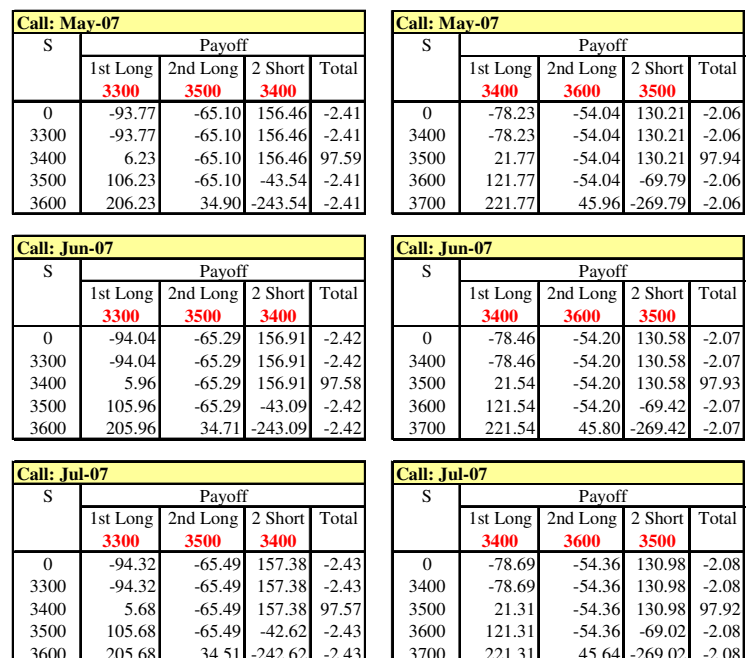

\begin{tabular}{|c|c|c|}
\hline & -242.62 & -2.43 \\
\hline
\end{tabular}
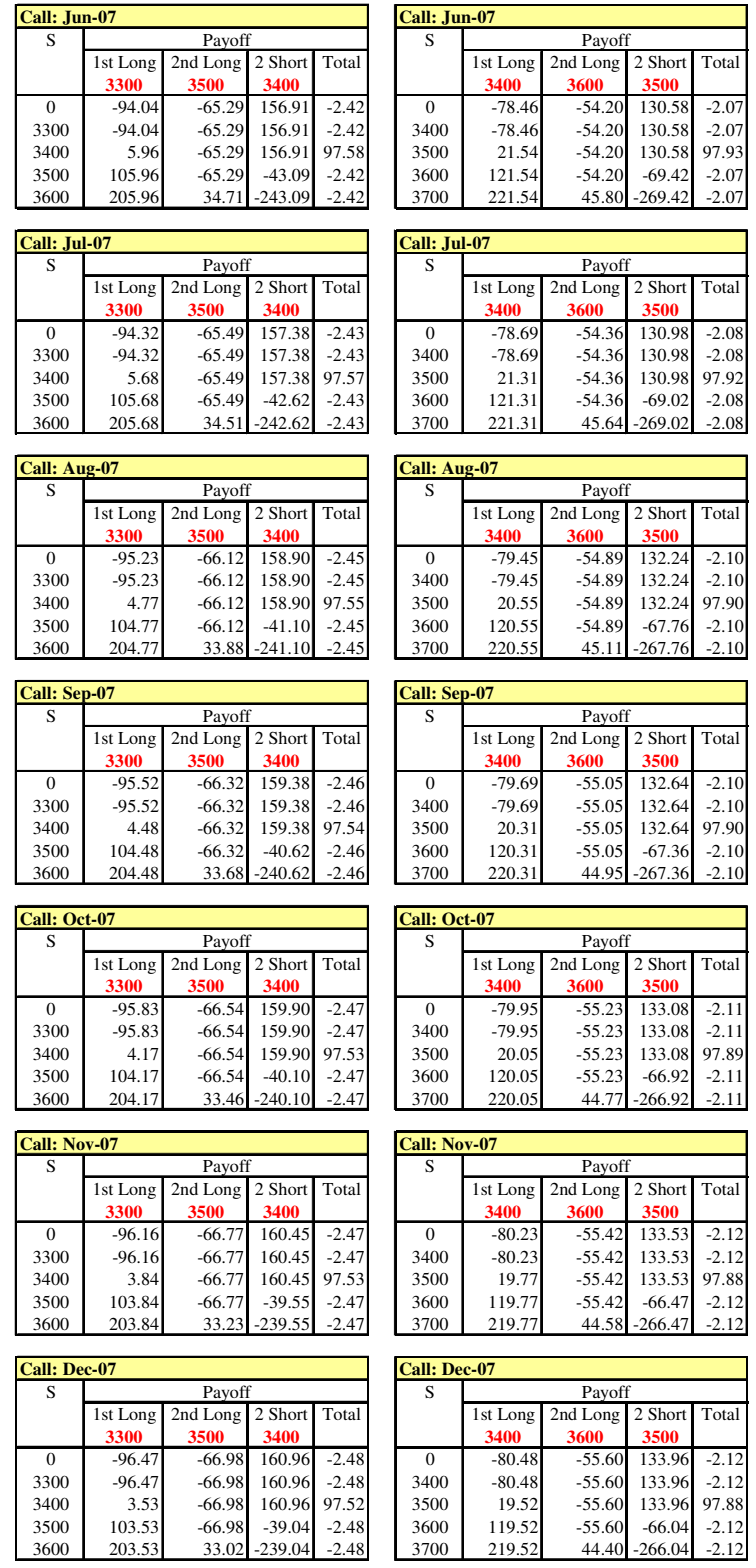


\section{Butterfly Spreads Using Put Options:}

If an investor feels it is unlikely that monthly average prices of copper on the LME will move significantly in the future (case A), creating a butterfly spread using put options on the date of data (22-Feb-05) was not optimal because the highest strike price for put options was $\$ 2,700 / t$ and chapter 6 results show that monthly average copper prices in 2006 and 2007 will evidently be over $\$ 3,200 / t$ and will not be around $\$ 2,700 /$ t.

\section{Case B: If a significant movement in the copper price is expected.}

\section{Butterfly Spreads Using Call Options:}

Table (7.35) constructs all possible butterfly spreads using call options for 2006 and 2007 if a significant movement in the copper price is expected. For each spread, two call options were bought at the middle strike price and call options with the lowest and highest strike prices were written (sold). There were four different butterfly spreads built using call options, so 96 butterfly spreads were available to be used on the date of data. Indeed, the butterfly spreads on table (7.35) are opposite strategies of the spreads on table (7.26).

The tables below construct the most profitable and effective four butterfly spread strategies each year in four different cases including monthly average copper price would expected to significantly move from each possible middle strike prices of $\$ 3,200, \$ 3,300$, $\$ 3,400$ and $\$ 3,500$. There are 8 most effective butterfly spreads using call options in 2006 and 2007 constructed. 


\section{Butterfly Spread for December 2006 (maximum profit)}

- Spot Price (22 Feb-05):

- Asian Call Options:

- Positions:
$\$ 3,367$

December-2006

Short Call @ 3100: $\quad$ premium $\$ 159.99$

Short Call @ 3300: $\quad$ premium $\$ 110.09$

Long 2 Calls @ 3200: premium $\$ 133.05$

Table 7.27: Profit from a Butterfly Spread (Dec-06 Call options, X: 3100/3200/3300)

\begin{tabular}{ccccc}
\hline $\begin{array}{c}\text { Copper price } \\
\text { Range }\end{array}$ & $\begin{array}{c}\text { Profit from } 1^{\text {st }} \\
\text { short call } \\
@ 3100\end{array}$ & $\begin{array}{c}\text { Profit from } 2^{\text {nd }} \\
\text { short call } \\
\text { @ 3300 }\end{array}$ & $\begin{array}{c}\text { Profit from 2 } \\
\text { long calls } \\
\text { @ 3200 }\end{array}$ & $\begin{array}{c}\text { Total } \\
\text { profit }\end{array}$ \\
\hline $3100>\mathrm{S}_{\mathrm{AVG}}$ & 171.22 & 117.81 & -284.77 & 4.26 \\
$3200>\mathrm{S}_{\mathrm{AVG}}>3100$ & $3271.22-\mathrm{S}_{\mathrm{AVG}}$ & 117.81 & -284.77 & $3104.26-\mathrm{S}_{\mathrm{AVG}}$ \\
$3300>\mathrm{S}_{\mathrm{AVG}}>3200$ & $3271.22-\mathrm{S}_{\mathrm{AVG}}$ & 117.81 & $2 \mathrm{~S}_{\mathrm{AVG}}-6684.77$ & $\mathrm{~S}_{\mathrm{AVG}}-3295.74$ \\
$\mathrm{~S}_{\mathrm{AVG}}>3300$ & $3271.22-\mathrm{S}_{\mathrm{AVG}}$ & $3417.81-\mathrm{S}_{\mathrm{AVG}}$ & $2 \mathrm{~S}_{\mathrm{AVG}}-6684.77$ & 4.26 \\
\hline
\end{tabular}

Profit is made when $S_{\mathrm{AVG}(\mathrm{Dec}-2006)}$ is less than $\$ 3,104.26$ or more than $\$ 3,295.74$ and is maximum of $\$ 4.26$ when $S_{\mathrm{AVG}(\operatorname{Dec}-2006)}$ is $\$ 3,100$ and less or $\$ 3,300$ and more.

\section{Butterfly Spread for December 2006 (maximum profit)}

- Spot Price (22 Feb-05):

- Asian Call Options:

- Positions:
$\$ 3,367$

December-2006

Short Call @ 3200: $\quad$ premium $\$ 133.05$

Short Call @ 3400: $\quad$ premium $\$ 90.67$

Long 2 Calls @ 3300: premium \$110.09

Table 7.28: Profit from a Butterfly Spread (Dec-06 Call options, X: 3200/3300/3400)

\begin{tabular}{ccccc}
\hline $\begin{array}{c}\text { Copper price } \\
\text { Range }\end{array}$ & $\begin{array}{c}\text { Profit from } 1^{\text {st }} \\
\text { short call } \\
\text { @ 3200 }\end{array}$ & $\begin{array}{c}\text { Profit from } 2^{\text {nd }} \\
\text { short call } \\
\text { @ } 3400\end{array}$ & $\begin{array}{c}\text { Profit from 2 } \\
\text { long calls } \\
@ 3 ~ 3300\end{array}$ & $\begin{array}{c}\text { Total } \\
\text { profit }\end{array}$ \\
\hline $3200>\mathrm{S}_{\mathrm{AVG}}$ & 142.39 & 97.03 & -235.63 & 3.79 \\
$3300>\mathrm{S}_{\mathrm{AVG}}>3200$ & $3342.39-\mathrm{S}_{\mathrm{AVG}}$ & 97.03 & -235.63 & $3203.79-\mathrm{S}_{\mathrm{AVG}}$ \\
$3400>\mathrm{S}_{\mathrm{AVG}}>3300$ & $3342.39-\mathrm{S}_{\mathrm{AVG}}$ & 97.03 & $2 \mathrm{~S}_{\mathrm{AVG}}-6835.63$ & $\mathrm{~S}_{\mathrm{AVG}}-3396.21$ \\
$\mathrm{~S}_{\mathrm{AVG}}>3400$ & $3342.39-\mathrm{S}_{\mathrm{AVG}}$ & $3497.03-\mathrm{S}_{\mathrm{AVG}}$ & $2 \mathrm{~S}_{\mathrm{AVG}}-6835.63$ & 3.79 \\
\hline
\end{tabular}

Profit is made when $S_{A V G(D e c-2006)}$ is less than $\$ 3,203.79$ or more than $\$ 3,396.21$ and is maximum of 3.79 when $S_{\mathrm{AVG}(\operatorname{Dec}-2006)}$ is $\$ 3,200$ and less or $\$ 3,400$ and more. 


\section{Butterfly Spread for December 2006 (maximum profit)}

- Spot Price (22 Feb-05):

- Asian Call Options:

- Positions:
$\$ 3,367$

December-2006

Short Call @ 3300: $\quad$ premium $\$ 110.09$

Short Call @ 3500: $\quad$ premium $\$ 74.35$

Long 2 Calls @ 3400: premium $\$ 90.67$

Table 7.29: Profit from a Butterfly Spread (Dec-06 Call options, X: 3300/3400/3500)

\begin{tabular}{ccccc}
\hline $\begin{array}{c}\text { Copper price } \\
\text { Range }\end{array}$ & $\begin{array}{c}\text { Profit from } 1^{\text {st }} \\
\text { short call } \\
@ 3300\end{array}$ & $\begin{array}{c}\text { Profit from } 2^{\text {nd }} \\
\text { short call } \\
@ 3500\end{array}$ & $\begin{array}{c}\text { Profit from 2 } \\
\text { long calls } \\
@ 3 ~ 3400\end{array}$ & $\begin{array}{c}\text { Total } \\
\text { profit }\end{array}$ \\
\hline $3300>\mathrm{S}_{\mathrm{AVG}}$ & 117.81 & 79.57 & -194.06 & 3.32 \\
$3400>\mathrm{S}_{\mathrm{AVG}}>3300$ & $3417.81-\mathrm{S}_{\mathrm{AVG}}$ & 79.57 & -194.06 & $3303.32-\mathrm{S}_{\mathrm{AVG}}$ \\
$3500>\mathrm{S}_{\mathrm{AVG}}>3400$ & $3417.81-\mathrm{S}_{\mathrm{AVG}}$ & 79.57 & $2 \mathrm{~S}_{\mathrm{AVG}}-6994.06$ & $\mathrm{~S}_{\mathrm{AVG}}-3496.68$ \\
$\mathrm{~S}_{\mathrm{AVG}}>3500$ & $3417.81-\mathrm{S}_{\mathrm{AVG}}$ & $3579.57-\mathrm{S}_{\mathrm{AVG}}$ & $2 \mathrm{~S}_{\mathrm{AVG}}-6994.06$ & 3.32 \\
\hline
\end{tabular}

Profit is made when $\mathrm{S}_{\mathrm{AVG}(\mathrm{Dec}-2006)}$ is less than $\$ 3,303.32$ or more than $\$ 3,496.68$ and is maximum of 3.32 when $\mathrm{S}_{\mathrm{AVG}(\operatorname{Dec}-2006)}$ is $\$ 3,300$ and less or $\$ 3,500$ and more.

\section{Butterfly Spread for December 2006 (maximum profit)}

- Spot Price (22 Feb-05):

- Asian Call Options:

- Positions:
$\$ 3,367$

December-2006

Short Call @ 3400: $\quad$ premium $\$ 90.67$

Short Call @ 3600: premium $\$ 60.72$

Long 2 Calls @ 3500: premium $\$ 74.35$

Table 7.30: Profit from a Butterfly Spread (Dec-06 Call options, X: 3400/3500/3600)

\begin{tabular}{ccccc}
\hline $\begin{array}{c}\text { Copper price } \\
\text { Range }\end{array}$ & $\begin{array}{c}\text { Profit from } 1^{\text {st }} \\
\text { short call } \\
@ 3400\end{array}$ & $\begin{array}{c}\text { Profit from } 2^{\text {nd }} \\
\text { short call } \\
\text { @ } 3600\end{array}$ & $\begin{array}{c}\text { Profit from 2 } \\
\text { long calls } \\
\text { @ 3500 }\end{array}$ & $\begin{array}{c}\text { Total } \\
\text { profit }\end{array}$ \\
\hline $3400>\mathrm{S}_{\mathrm{AVG}}$ & 97.03 & 64.98 & -159.13 & \\
$3500>\mathrm{S}_{\mathrm{AVG}}>3400$ & $3497.03-\mathrm{S}_{\mathrm{AVG}}$ & 64.98 & -159.13 & $3402.88-\mathrm{S}_{\mathrm{AVG}}$ \\
$3600>\mathrm{S}_{\mathrm{AVG}}>3500$ & $3497.03-\mathrm{S}_{\mathrm{AVG}}$ & 64.98 & $2 \mathrm{~S}_{\mathrm{AVG}}-7159.13$ & $\mathrm{~S}_{\mathrm{AVG}}-3597.12$ \\
$\mathrm{~S}_{\mathrm{AVG}}>3600$ & $3497.03-\mathrm{S}_{\mathrm{AVG}}$ & $3664.98-\mathrm{S}_{\mathrm{AVG}}$ & $2 \mathrm{~S}_{\mathrm{AVG}}-7159.13$ & 2.88 \\
\hline
\end{tabular}

Profit is made when $S_{A V G(D e c-2006)}$ is less than $\$ 3,402.88$ or more than $\$ 3,597.12$ and is maximum of 2.88 when $S_{\mathrm{AVG}(\operatorname{Dec}-2006)}$ is $\$ 3,400$ and less or $\$ 3,600$ and more. 
Figure (7.22): Butterfly Spread, Call Options Dec-06, X: 3100/3200/3300

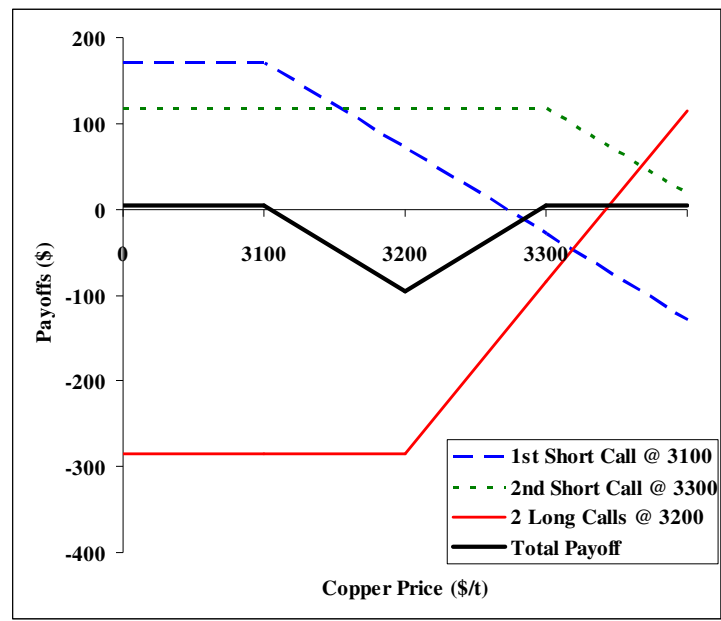

Figure (7.23): Butterfly Spread, Call Options Dec-06, X: 3200/3300/3400

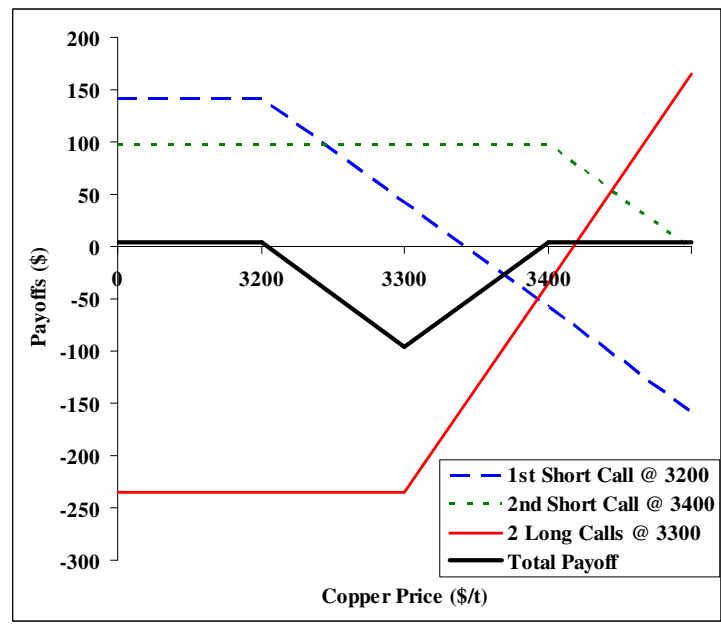

Figure (7.24): Butterfly Spread, Call Options Dec-06, X: 3300/3400/3500

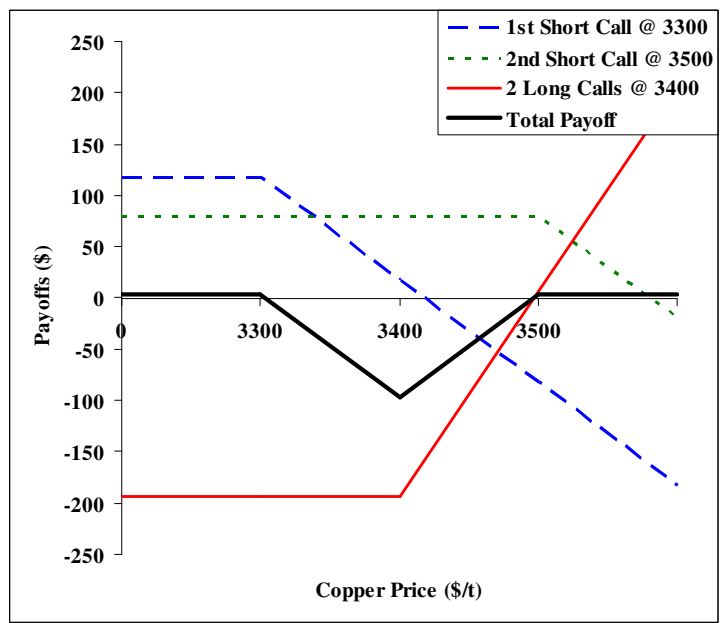

Figure (7.25): Butterfly Spread, Call Options Dec-06, X: 3400/3500/3600

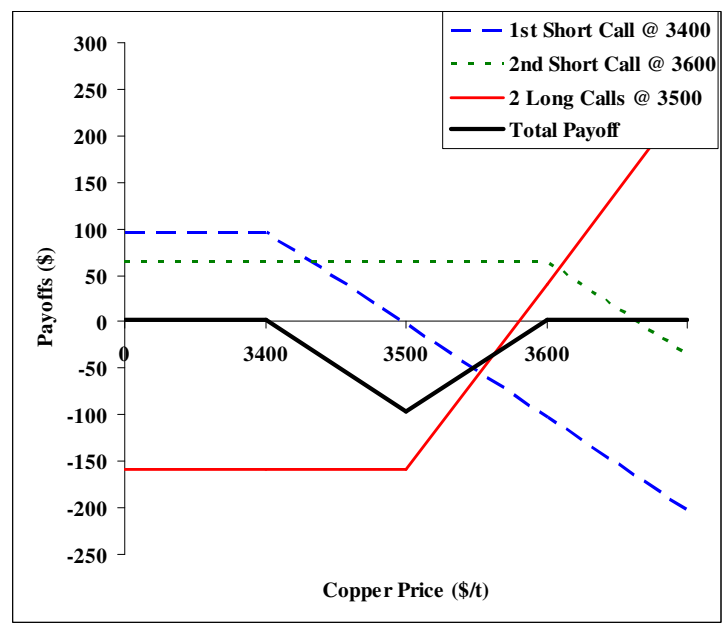

Comparing the 48 butterfly spreads, which were created using call options in 2006 , the spreads on Dec-06 calls would be more effective because if the price fluctuates in either direction from the middle strike prices $(\$ 3200, \$ 3300, \$ 3400$ and $\$ 3500)$ the profit would be greatest for December 2006 spreads. Moreover, the loss would be no more than \$95.74, \$96.21, \$96.68 and \$97.12 for December 2006 spreads if the price settles around the middle strike prices. 


\section{Butterfly Spread for December 2007 (maximum profit)}

- Spot Price (22 Feb-05):

- Asian Call Options:

- Positions:
$\$ 3,367$

December-2007

Short Call @ 3100: $\quad$ premium $\$ 122.86$

Short Call @ 3300: $\quad$ premium $\$ 86.30$

Long 2 Calls @ 3200: $\quad$ premium $\$ 103.13$

Table 7.31: Profit from a Butterfly Spread (Dec-07 Call options, X: 3100/3200/3300)

\begin{tabular}{ccccc}
\hline $\begin{array}{c}\text { Copper price } \\
\text { Range }\end{array}$ & $\begin{array}{c}\text { Profit from } 1^{\text {st }} \\
\text { short call } \\
@ 3100\end{array}$ & $\begin{array}{c}\text { Profit from } 2^{\text {nd }} \\
\text { short call } \\
@ 3300\end{array}$ & $\begin{array}{c}\text { Profit from 2 } \\
\text { long calls } \\
\text { @ 3200 }\end{array}$ & $\begin{array}{c}\text { Total } \\
\text { profit }\end{array}$ \\
\hline $3100>\mathrm{S}_{\mathrm{AVG}}$ & 137.33 & 96.47 & -230.56 & 3.24 \\
$3200>\mathrm{S}_{\mathrm{AVG}}>3100$ & $3237.33-\mathrm{S}_{\mathrm{AVG}}$ & 96.47 & -230.56 & $3103.24-\mathrm{S}_{\mathrm{AVG}}$ \\
$3300>\mathrm{S}_{\mathrm{AVG}}>3200$ & $3237.33-\mathrm{S}_{\mathrm{AVG}}$ & 96.47 & $2 \mathrm{~S}_{\mathrm{AVG}}-6630.56$ & $\mathrm{~S}_{\mathrm{AVG}}-3296.76$ \\
$\mathrm{~S}_{\mathrm{AVG}}>3300$ & $3237.33-\mathrm{S}_{\mathrm{AVG}}$ & $3396.47-\mathrm{S}_{\mathrm{AVG}}$ & $2 \mathrm{~S}_{\mathrm{AVG}}-6630.56$ & 3.24 \\
\hline
\end{tabular}

Profit is made when $S_{A V G(D e c-2007)}$ is less than $\$ 3,103.24$ or more than $\$ 3,296.76$ and is maximum of $\$ 3.24$ when $S_{\mathrm{AVG}(\operatorname{Dec}-2007)}$ is $\$ 3,100$ and less or $\$ 3,300$ and more.

\section{Butterfly Spread for December 2007 (maximum profit)}

- Spot Price (22 Feb-05):

- Asian Call Options:

- Positions:
$\$ 3,367$

December-2007

Short Call @ 3200: $\quad$ premium $\$ 103.13$

Short Call@ 3400:_ premium $\$ 72.00$

Long 2 Calls@3300: premium $\$ 86.30$

Table 7.32: Profit from a Butterfly Spread (Dec-07 Call options, X: 3200/3300/3400)

\begin{tabular}{ccccc}
\hline $\begin{array}{c}\text { Copper price } \\
\text { Range }\end{array}$ & $\begin{array}{c}\text { Profit from } 1^{\text {st }} \\
\text { short call } \\
@ 3200\end{array}$ & $\begin{array}{c}\text { Profit from } 2^{\text {nd }} \\
\text { short call } \\
\text { @ } 3400\end{array}$ & $\begin{array}{c}\text { Profit from } 2 \\
\text { long calls } \\
\text { @ 3300 }\end{array}$ & $\begin{array}{c}\text { Total } \\
\text { profit }\end{array}$ \\
\hline $3200>\mathrm{S}_{\mathrm{AVG}}$ & 115.28 & 80.48 & -192.93 & \\
$3300>\mathrm{S}_{\mathrm{AVG}}>3200$ & $3315.28-\mathrm{S}_{\mathrm{AVG}}$ & 80.48 & -192.93 & $3202.83-\mathrm{S}_{\mathrm{AVG}}$ \\
$3400>\mathrm{S}_{\mathrm{AVG}}>3300$ & $3315.28-\mathrm{S}_{\mathrm{AVG}}$ & 80.48 & $2 \mathrm{~S}_{\mathrm{AVG}}-6792.93$ & $\mathrm{~S}_{\mathrm{AVG}}-3397.17$ \\
$\mathrm{~S}_{\mathrm{AVG}}>3400$ & $3315.28-\mathrm{S}_{\mathrm{AVG}}$ & $3480.48-\mathrm{S}_{\mathrm{AVG}}$ & $2 \mathrm{~S}_{\mathrm{AVG}}-6792.93$ & 2.83 \\
\hline
\end{tabular}

Profit is made when $S_{A V G(D e c-2007)}$ is less than $\$ 3,202.83$ or more than $\$ 3,397.17$ and is maximum of 2.83 when $S_{\mathrm{AVG}(\operatorname{Dec}-2007)}$ is $\$ 3,200$ and less or $\$ 3,400$ and more. 


\section{Butterfly Spread for December 2007 (maximum profit)}

- Spot Price (22 Feb-05):

- Asian Call Options:

- Positions:
$\$ 3,367$

December-2007

Short Call @ 3300: $\quad$ premium $\$ 86.30$

Short Call @ 3500: $\quad$ premium $\$ 59.92$

Long 2 Calls @ 3400: premium $\$ 72.00$

Table 7.33: Profit from a Butterfly Spread (Dec-07 Call options, X: 3300/3400/3500)

\begin{tabular}{ccccc}
\hline $\begin{array}{c}\text { Copper price } \\
\text { Range }\end{array}$ & $\begin{array}{c}\text { Profit from } 1^{\text {st }} \\
\text { short call } \\
@ 3300\end{array}$ & $\begin{array}{c}\text { Profit from } 2^{\text {nd }} \\
\text { short call } \\
@ 3500\end{array}$ & $\begin{array}{c}\text { Profit from 2 } \\
\text { long calls } \\
@ 3 ~ 3400\end{array}$ & $\begin{array}{c}\text { Total } \\
\text { profit }\end{array}$ \\
\hline $3300>\mathrm{S}_{\mathrm{AVG}}$ & 96.47 & 66.98 & -160.96 & 2.49 \\
$3400>\mathrm{S}_{\mathrm{AVG}}>3300$ & $3396.47-\mathrm{S}_{\mathrm{AVG}}$ & 66.98 & -160.96 & $3302.49-\mathrm{S}_{\mathrm{AVG}}$ \\
$3500>\mathrm{S}_{\mathrm{AVG}}>3400$ & $3396.47-\mathrm{S}_{\mathrm{AVG}}$ & 66.98 & $2 \mathrm{~S}_{\mathrm{AVG}}-6960.96$ & $\mathrm{~S}_{\mathrm{AVG}}-3497.51$ \\
$\mathrm{~S}_{\mathrm{AVG}}>3500$ & $3396.47-\mathrm{S}_{\mathrm{AVG}}$ & $3566.98-\mathrm{S}_{\mathrm{AVG}}$ & $2_{\mathrm{S}} \mathrm{S}_{\mathrm{AVG}}-6960.96$ & 2.49 \\
\hline
\end{tabular}

Profit is made when $\mathrm{S}_{\mathrm{AVG}(\mathrm{Dec}-2007)}$ is less than $\$ 3,302.49$ or more than $\$ 3,497.51$ and is maximum of 2.49 when $S_{\mathrm{AVG}(\mathrm{Dec}-2007)}$ is $\$ 3,300$ and less or $\$ 3,500$ and more.

\section{Butterfly Spread for December 2007 (maximum profit)}

- Spot Price (22 Feb-05):

- Asian Call Options:

- Positions:
$\$ 3,367$

December-2007

Short Call @ 3400: $\quad$ premium $\$ 72.00$

Short Call @ 3600: premium $\$ 49.74$

Long 2 Calls @ 3500: premium $\$ 59.92$

Table 7.34: Profit from a Butterfly Spread (Dec-07 Call options, X: 3400/3500/3600)

\begin{tabular}{ccccc}
\hline $\begin{array}{c}\text { Copper price } \\
\text { Range }\end{array}$ & $\begin{array}{c}\text { Profit from } 1^{\text {st }} \\
\text { short call } \\
@ 3400\end{array}$ & $\begin{array}{c}\text { Profit from } 2^{\text {nd }} \\
\text { short call } \\
\text { @ } 3600\end{array}$ & $\begin{array}{c}\text { Profit from 2 } \\
\text { long calls } \\
@ 3 ~ 3500\end{array}$ & $\begin{array}{c}\text { Total } \\
\text { profit }\end{array}$ \\
\hline $3400>\mathrm{S}_{\mathrm{AVG}}$ & 80.48 & 55.60 & -133.96 & \\
$3500>\mathrm{S}_{\mathrm{AVG}}>3400$ & $3480.48-\mathrm{S}_{\mathrm{AVG}}$ & 55.60 & -133.96 & $3402.12-\mathrm{S}_{\mathrm{AVG}}$ \\
$3600>\mathrm{S}_{\mathrm{AVG}}>3500$ & $3480.48-\mathrm{S}_{\mathrm{AVG}}$ & 55.60 & $2 \mathrm{~S}_{\mathrm{AVG}}-7133.96$ & $\mathrm{~S}_{\mathrm{AVG}}-3597.88$ \\
$\mathrm{~S}_{\mathrm{AVG}}>3600$ & $3480.48-\mathrm{S}_{\mathrm{AVG}}$ & $3655.60-\mathrm{S}_{\mathrm{AVG}}$ & $2 \mathrm{~S}_{\mathrm{AVG}}-7133.96$ & 2.12 \\
\hline
\end{tabular}

Profit is made when $S_{A V G(D e c-2007)}$ is less than $\$ 3,402.12$ or more than $\$ 3,597.88$ and is maximum of 2.12 when $S_{\mathrm{AVG}(\operatorname{Dec}-2007)}$ is $\$ 3,400$ and less or $\$ 3,600$ and more. 
Figure (7.26): Butterfly Spread, Call Options Dec-07, X: 3100/3200/3300

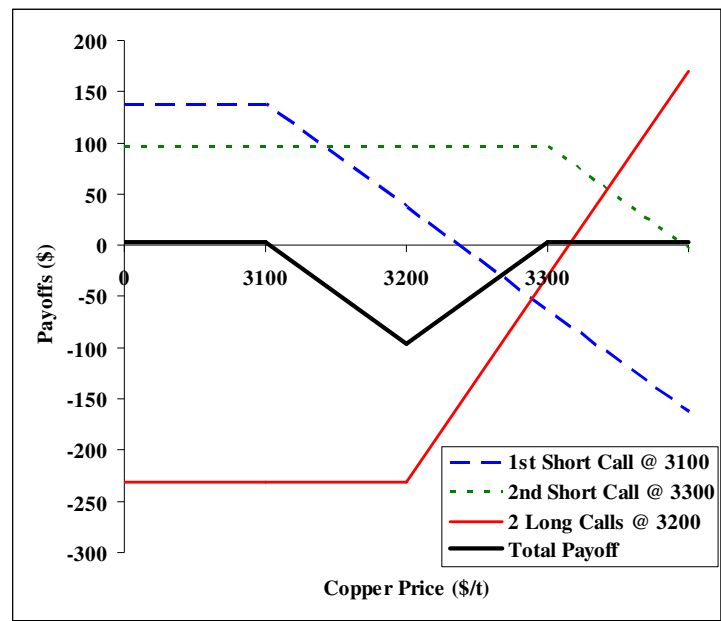

Figure (7.27): Butterfly Spread, Call Options Dec-07, X: 3200/3300/3400

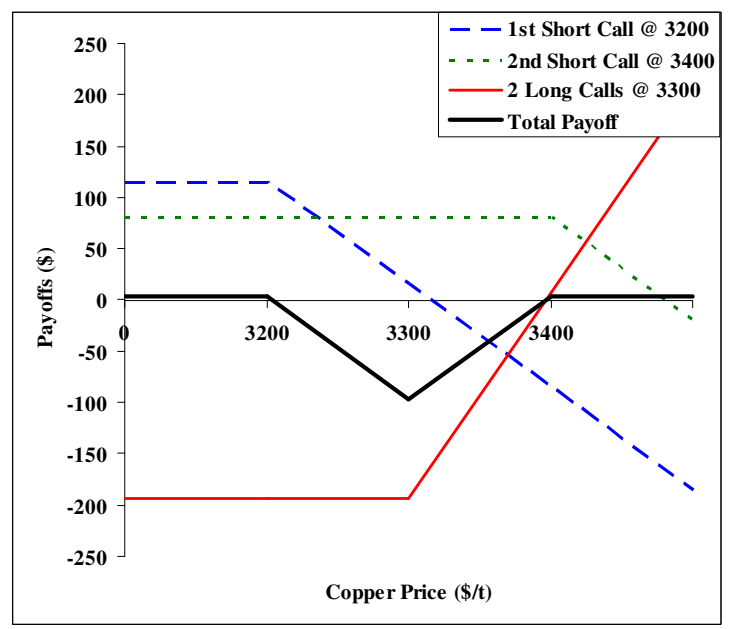

Figure (7.28): Butterfly Spread, Call Options Dec-07, X: 3300/3400/3500

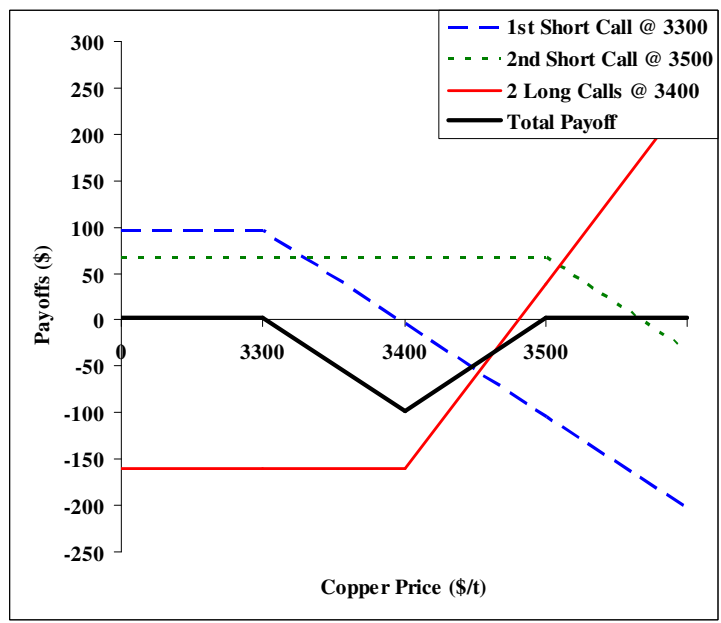

Figure (7.29): Butterfly Spread, Call Options Dec-07, X: 3400/3500/3600

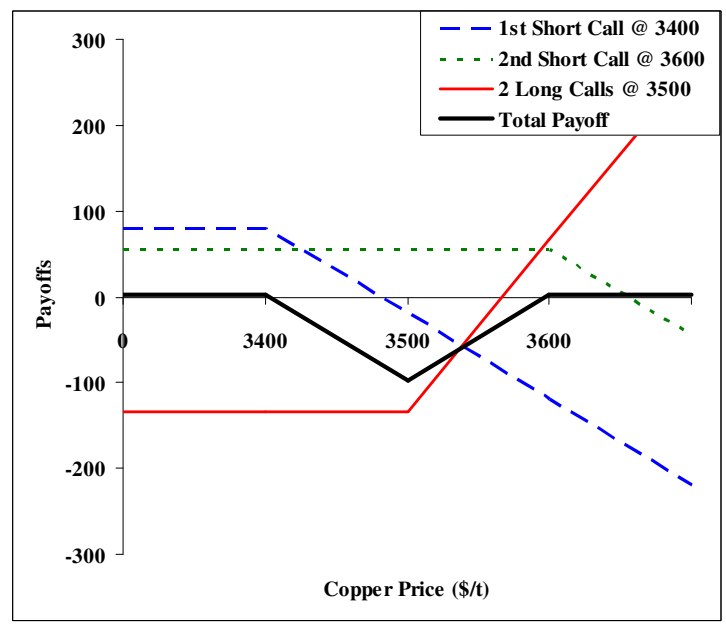

Comparing these 48 butterfly spreads, which were created using call options in 2007 , the spreads on Dec-07 calls would be more effective because if the price fluctuates in either direction from the middle strike prices the profit would be greatest for December 2007 spreads. Moreover, the loss would be no more than $\$ 96.76, \$ 97.17, \$ 97.52$ and \$97.88 for December 2007 spreads if the price settles around the middle strike prices. 
Table 7.35: Butterfly Spreads Using Call Options for 2006 and 2007 (Case B)
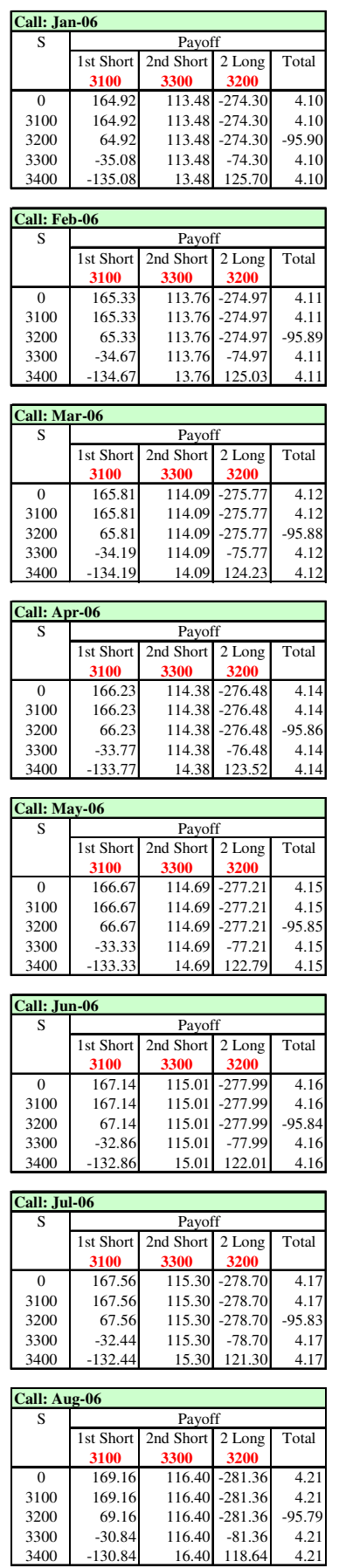
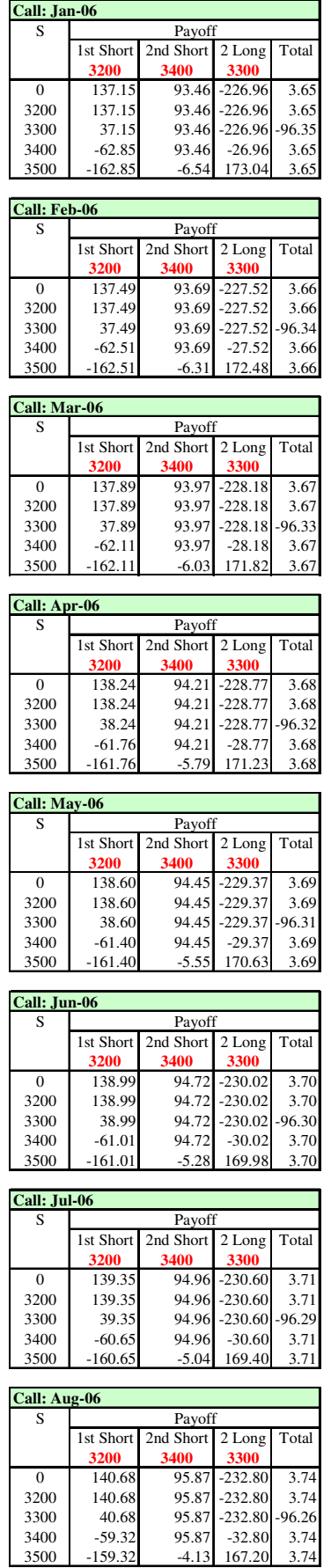
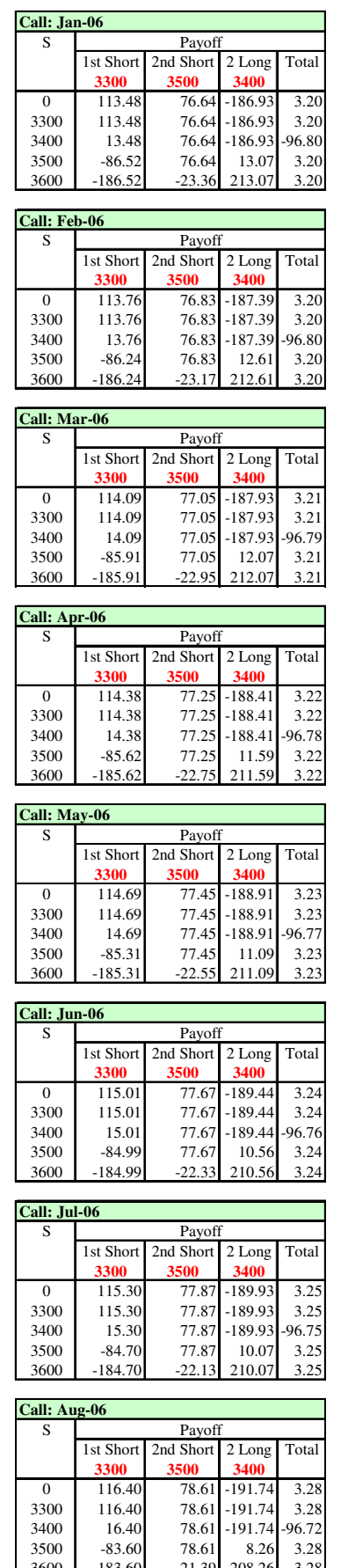
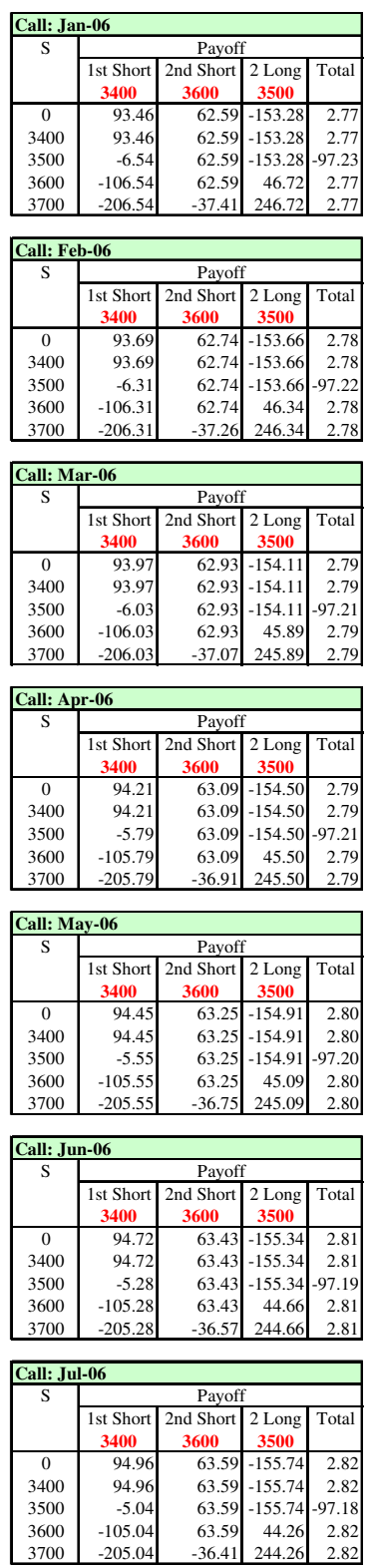

\begin{tabular}{|c|r|r|r|r|}
\hline \multicolumn{5}{|c|}{ Call: Aug-06 } \\
\hline S & \multicolumn{4}{|c|}{ Payoff } \\
\cline { 2 - 5 } & 1st Short & 2nd Short & 2 Long & Total \\
& \multicolumn{1}{|c|}{3400} & \multicolumn{1}{c|}{3600} & $\mathbf{3 5 0 0}$ & \\
\hline 0 & 95.87 & 64.20 & -157.22 & 2.84 \\
3400 & 95.87 & 64.20 & -157.22 & 2.84 \\
3500 & -4.13 & 64.20 & -157.22 & -97.16 \\
3600 & -104.13 & 64.20 & 42.78 & 2.84 \\
3700 & -204.13 & -35.80 & 242.78 & 2.84 \\
\hline
\end{tabular}


Table 7.35 continued

\begin{tabular}{|c|r|r|r|r|}
\hline Call: Sep-06 \\
\hline S & \multicolumn{4}{|c|}{ Payoff } \\
\cline { 2 - 5 } & \multicolumn{1}{|c|}{ 1st Short } & 2nd Short & 2 Long & Total \\
& \multicolumn{1}{|c|}{$\mathbf{3 1 0 0}$} & \multicolumn{1}{c|}{$\mathbf{3 3 0 0}$} & $\mathbf{3 2 0 0}$ & \\
\hline 0 & 169.65 & 116.74 & -282.17 & 4.22 \\
3100 & 169.65 & 116.74 & -282.17 & 4.22 \\
3200 & 69.65 & 116.74 & -282.17 & -95.78 \\
3300 & -30.35 & 116.74 & -82.17 & 4.22 \\
3400 & -130.35 & 16.74 & 117.83 & 4.22 \\
\hline
\end{tabular}

\begin{tabular}{|c|r|r|r|r|}
\hline \multicolumn{4}{|l|}{ Call: Oct-06 } \\
\hline \multirow{2}{*}{ S } & \multicolumn{4}{|c|}{ Payoff } \\
\cline { 2 - 5 } & 1st Short & 2nd Short & 2 Long & Total \\
& \multicolumn{1}{|c|}{3100} & \multicolumn{1}{|c|}{$\mathbf{3 3 0 0}$} & $\mathbf{3 2 0 0}$ & \\
\hline 0 & 170.16 & 117.09 & -283.02 & 4.23 \\
3100 & 170.16 & 117.09 & -283.02 & 4.23 \\
3200 & 70.16 & 117.09 & -283.02 & -95.77 \\
3300 & -29.84 & 117.09 & -83.02 & 4.23 \\
3400 & -129.84 & 17.09 & 116.98 & 4.23 \\
\hline
\end{tabular}

\begin{tabular}{|c|r|r|r|r|}
\hline \multicolumn{5}{|c|}{ Call: Nov-06 } \\
\hline S & \multicolumn{4}{|c|}{ Payoff } \\
\cline { 2 - 5 } & 1st Short & 2nd Short & 2 Long & Total \\
& \multicolumn{1}{c|}{3100} & \multicolumn{1}{c|}{$\mathbf{3 3 0 0}$} & $\mathbf{3 2 0 0}$ & \\
\hline 0 & 170.70 & 117.46 & -283.92 & 4.25 \\
3100 & 170.70 & 117.46 & -283.92 & 4.25 \\
3200 & 70.70 & 117.46 & -283.92 & -95.75 \\
3300 & -29.30 & 117.46 & -83.92 & 4.25 \\
3400 & -129.30 & 17.46 & 116.08 & 4.25 \\
\hline
\end{tabular}
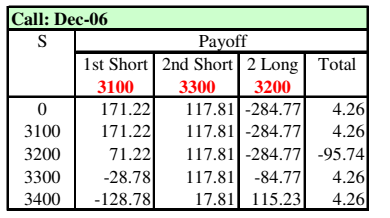

\begin{tabular}{|c|r|r|r|r|}
\hline \multicolumn{5}{|l|}{ Call: Jan-07 } \\
\hline \multirow{2}{*}{ S } & \multicolumn{4}{|c|}{ Payoff } \\
\cline { 2 - 5 } & \multicolumn{1}{|c|}{ 1st Short } & 2nd Short & 2 Long & Total \\
& \multicolumn{1}{|c|}{$\mathbf{3 3 0 0}$} & $\mathbf{3 2 0 0}$ & \\
\hline 0 & 131.87 & 92.63 & -221.39 & 3.11 \\
3100 & 131.87 & 92.63 & -221.39 & 3.11 \\
3200 & 31.87 & 92.63 & -221.39 & -96.89 \\
3300 & -68.13 & 92.63 & -21.39 & 3.11 \\
3400 & -168.13 & -7.37 & 178.61 & 3.11 \\
\hline
\end{tabular}

\begin{tabular}{|c|c|c|c|r|}
\hline \multicolumn{4}{|l|}{ Call: Feb-07 } \\
\hline S & \multicolumn{3}{|c|}{ Payoff } \\
\cline { 2 - 5 } & \multicolumn{1}{|c|}{ 1st Short } & 2nd Short & 2 Long & Total \\
\hline 0 & \multicolumn{1}{|c|}{132.24} & 92.89 & -222.01 & 3.12 \\
3100 & 132.24 & 92.89 & -222.01 & 3.12 \\
3200 & 32.24 & 92.89 & -222.01 & -96.88 \\
3300 & -67.76 & 92.89 & -22.01 & 3.12 \\
3400 & -167.76 & -7.11 & 177.99 & 3.12 \\
\hline
\end{tabular}

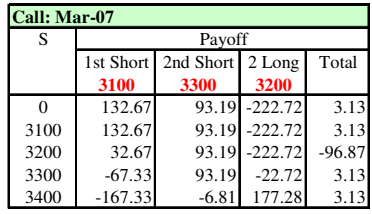

\begin{tabular}{|c|r|r|r|r|}
\hline \multicolumn{5}{|l|}{ Call: Apr-07 } \\
\hline $\mathrm{S}$ & \multicolumn{4}{|c|}{ Payoff } \\
\cline { 2 - 5 } & 1st Short & 2nd Short & 2 Long & Total \\
& \multicolumn{1}{|c|}{$\mathbf{1 1 0 0}$} & \multicolumn{1}{|c|}{$\mathbf{3 3 0 0}$} & $\mathbf{3 2 0 0}$ & \\
\hline 0 & 133.05 & 93.46 & -223.37 & 3.14 \\
3100 & 133.05 & 93.46 & -223.37 & 3.14 \\
3200 & 33.05 & 93.46 & -223.37 & -96.86 \\
3300 & -66.95 & 93.46 & -23.37 & 3.14 \\
3400 & -166.95 & -6.54 & 176.63 & 3.14 \\
\hline
\end{tabular}
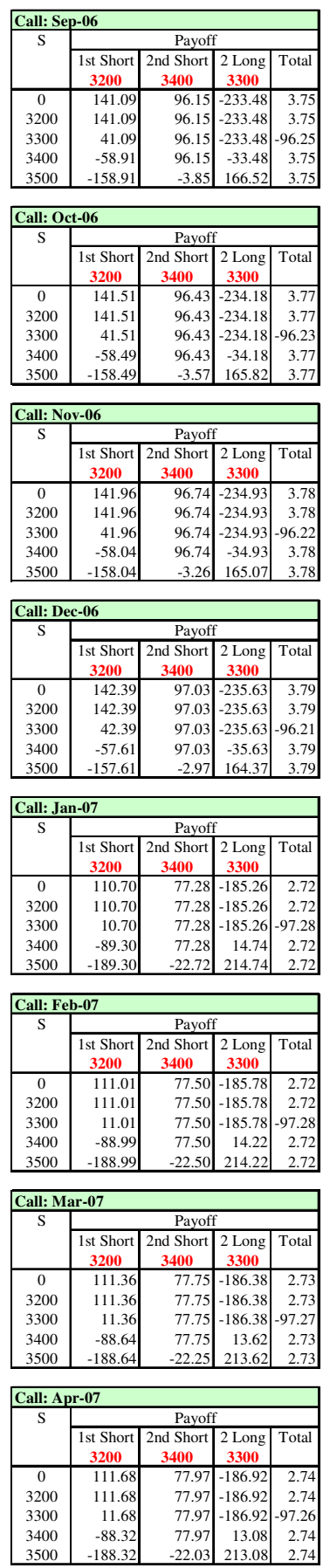
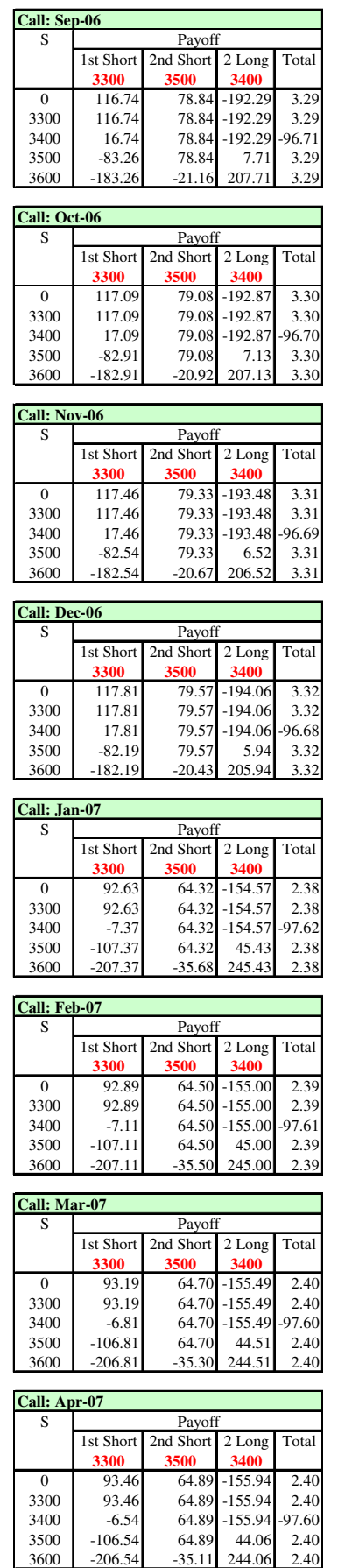
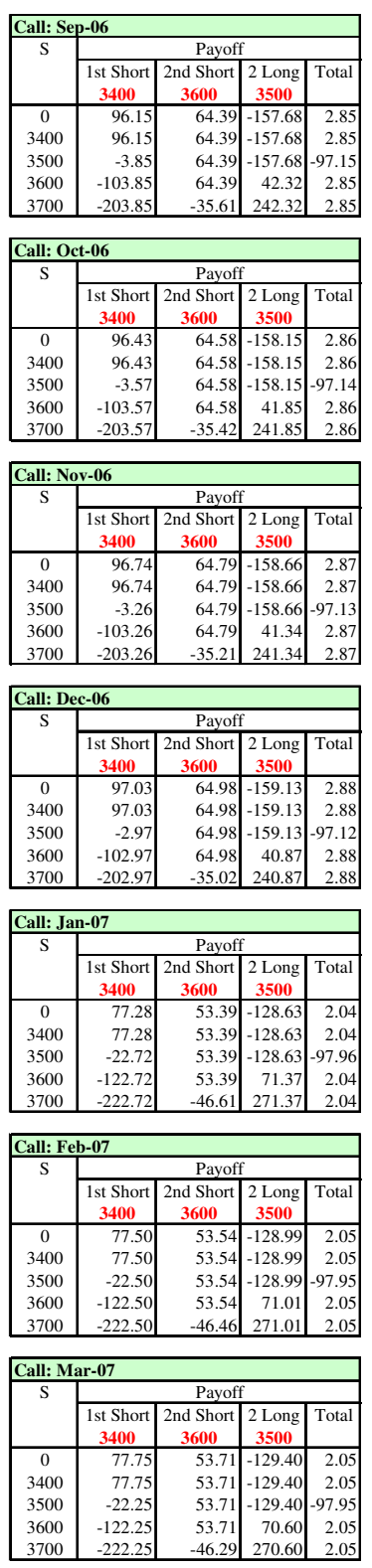

\begin{tabular}{|c|c|c|c|c|}
\hline \multicolumn{5}{|c|}{ Call: Apr-07 } \\
\hline \multirow[t]{2}{*}{$\mathrm{S}$} & \multicolumn{4}{|c|}{ Payoff } \\
\hline & $\begin{array}{c}\text { 1st Short } \\
3400\end{array}$ & $\begin{array}{c}\text { 2nd Short } \\
3600\end{array}$ & $\begin{array}{c}2 \text { Long } \\
3500\end{array}$ & \begin{tabular}{|l|} 
Total \\
\end{tabular} \\
\hline 0 & 77.97 & $\begin{array}{lll}53.87 \\
\end{array}$ & \begin{tabular}{|l|}
-129.78 \\
\end{tabular} & 2.06 \\
\hline 3400 & 77.97 & 53.87 & -129.78 & 2.06 \\
\hline 3500 & -22.03 & 53.87 & -129.78 & \begin{tabular}{|l|l|}
-97.94 \\
\end{tabular} \\
\hline 3600 & -122.03 & 53.87 & 70.22 & 2.06 \\
\hline 3700 & -222.03 & -46.13 & 270.22 & 2.06 \\
\hline
\end{tabular}


Table 7.35 continued
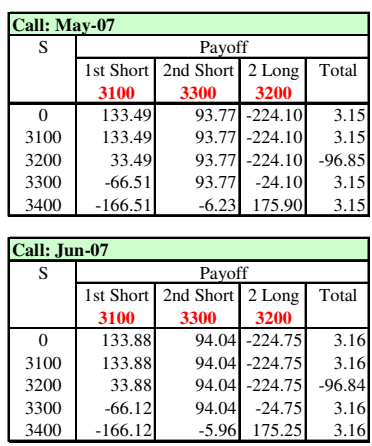

\begin{tabular}{|c|c|c|c|c|}
\hline \multicolumn{5}{|c|}{ Call: Jul-07 } \\
\hline \multirow[t]{2}{*}{ S } & \multicolumn{4}{|c|}{ Payoff } \\
\hline & \begin{tabular}{|c|} 
1st Short \\
3100
\end{tabular} & $\begin{array}{c}\text { 2nd Short } \\
3300\end{array}$ & \begin{tabular}{|c}
2 Long \\
3200
\end{tabular} & Total \\
\hline 0 & 134.28 & 94.32 & -225.43 & 3.17 \\
\hline 3100 & 134.28 & 94.32 & -225.43 & 3.17 \\
\hline 3200 & 34.28 & 94.32 & -225.43 & -96.83 \\
\hline 3300 & -65.72 & 94.32 & -25.43 & 3.17 \\
\hline 3400 & & & $1 / 4$ & \\
\hline
\end{tabular}

\begin{tabular}{|c|r|r|r|r|}
\hline \multicolumn{4}{|c|}{ Call: Aug-07 } \\
\hline $\mathrm{S}$ & \multicolumn{4}{|c|}{ Payoff } \\
\cline { 2 - 5 } & \multicolumn{1}{|c|}{ 1st Short } & 2nd Short & 2 Long & Total \\
& \multicolumn{1}{|c|}{$\mathbf{3 1 0 0}$} & $\mathbf{3 3 0 0}$ & $\mathbf{3 2 0 0}$ & \\
\hline 0 & 135.57 & 95.23 & -227.61 & 3.20 \\
3100 & 135.57 & 95.23 & -227.61 & 3.20 \\
3200 & 35.57 & 95.23 & -227.61 & -96.80 \\
3300 & -64.43 & 95.23 & -27.61 & 3.20 \\
3400 & -164.43 & -4.77 & 172.39 & 3.20 \\
\hline
\end{tabular}

\begin{tabular}{|c|r|r|r|r|}
\hline \multicolumn{6}{|c|}{ Call: Sep-07 } \\
\hline $\mathrm{S}$ & \multicolumn{4}{|c|}{ Payoff } \\
\cline { 2 - 5 } & \multicolumn{1}{|c|}{ 1st Short } & 2nd Short & 2 Long & Total \\
& \multicolumn{1}{|c|}{$\mathbf{3 3 0 0}$} & $\mathbf{3 2 0 0}$ & \\
\hline 0 & 135.98 & 95.52 & -228.29 & 3.21 \\
3100 & 135.98 & 95.52 & -228.29 & 3.21 \\
3200 & 35.98 & 95.52 & -228.29 & -96.79 \\
3300 & -64.02 & 95.52 & -28.29 & 3.21 \\
3400 & -164.02 & -4.48 & 171.71 & 3.21 \\
\hline
\end{tabular}

\begin{tabular}{|c|c|c|c|c|}
\hline \multicolumn{5}{|c|}{ Call: Oct-07 } \\
\hline \multirow[t]{2}{*}{$\mathrm{S}$} & \multicolumn{4}{|c|}{ Payoff } \\
\hline & $\begin{array}{c}\text { 1st Short } \\
3300\end{array}$ & 2nd Short & $\begin{array}{r}2 \text { Long } \\
3200\end{array}$ & Total \\
\hline 0 & 136.43 & 95.83 & -229.04 & 3.22 \\
\hline 3100 & 136.43 & 95.83 & -229.04 & 3.22 \\
\hline 3200 & 36.43 & 95.83 & -229.04 & -96.78 \\
\hline 3300 & -63.57 & 95.83 & -29.04 & 3.22 \\
\hline 3400 & -16 & & 170.96 & 3. \\
\hline
\end{tabular}

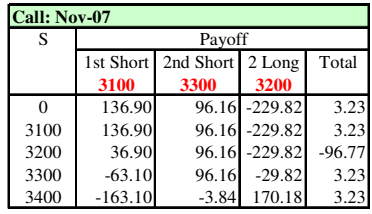

\begin{tabular}{|c|c|c|c|c|}
\hline \multicolumn{5}{|c|}{ Call: Dec- 07} \\
\hline \multirow[t]{2}{*}{$\mathrm{S}$} & \multicolumn{4}{|c|}{ Payoff } \\
\hline & \begin{tabular}{|c|} 
1st Short \\
3100
\end{tabular} & $\begin{array}{c}\text { 2nd Short } \\
3300\end{array}$ & \begin{tabular}{|c|}
2 Long \\
3200
\end{tabular} & Total \\
\hline 0 & 137.33 & 96.47 & -230.56 & 3.24 \\
\hline 3100 & 137.33 & 96.47 & -230.56 & 3.24 \\
\hline 3200 & 37.33 & 96.47 & -230.56 & -96.76 \\
\hline 3300 & -62.67 & 96.47 & -30.56 & 3.24 \\
\hline U & 162.6 & 5.5 & 109 & 3.2 \\
\hline
\end{tabular}
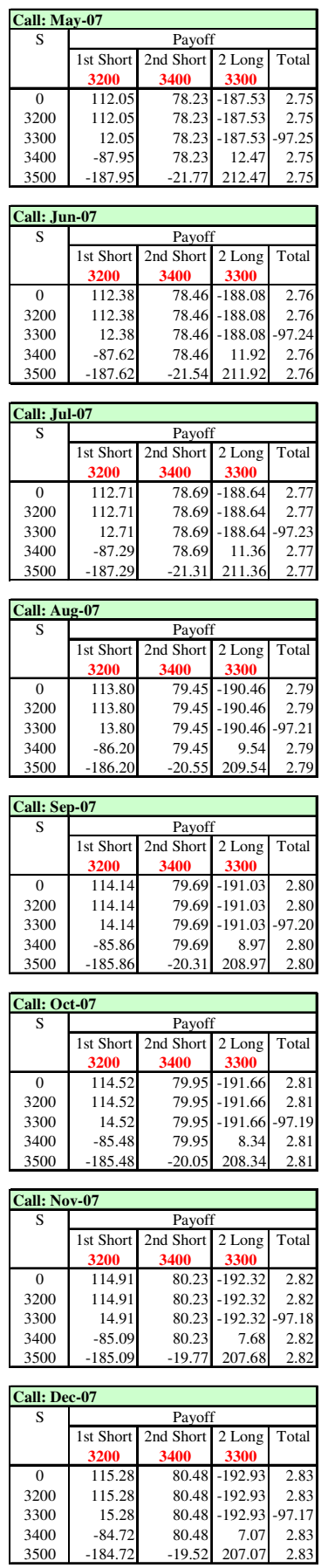
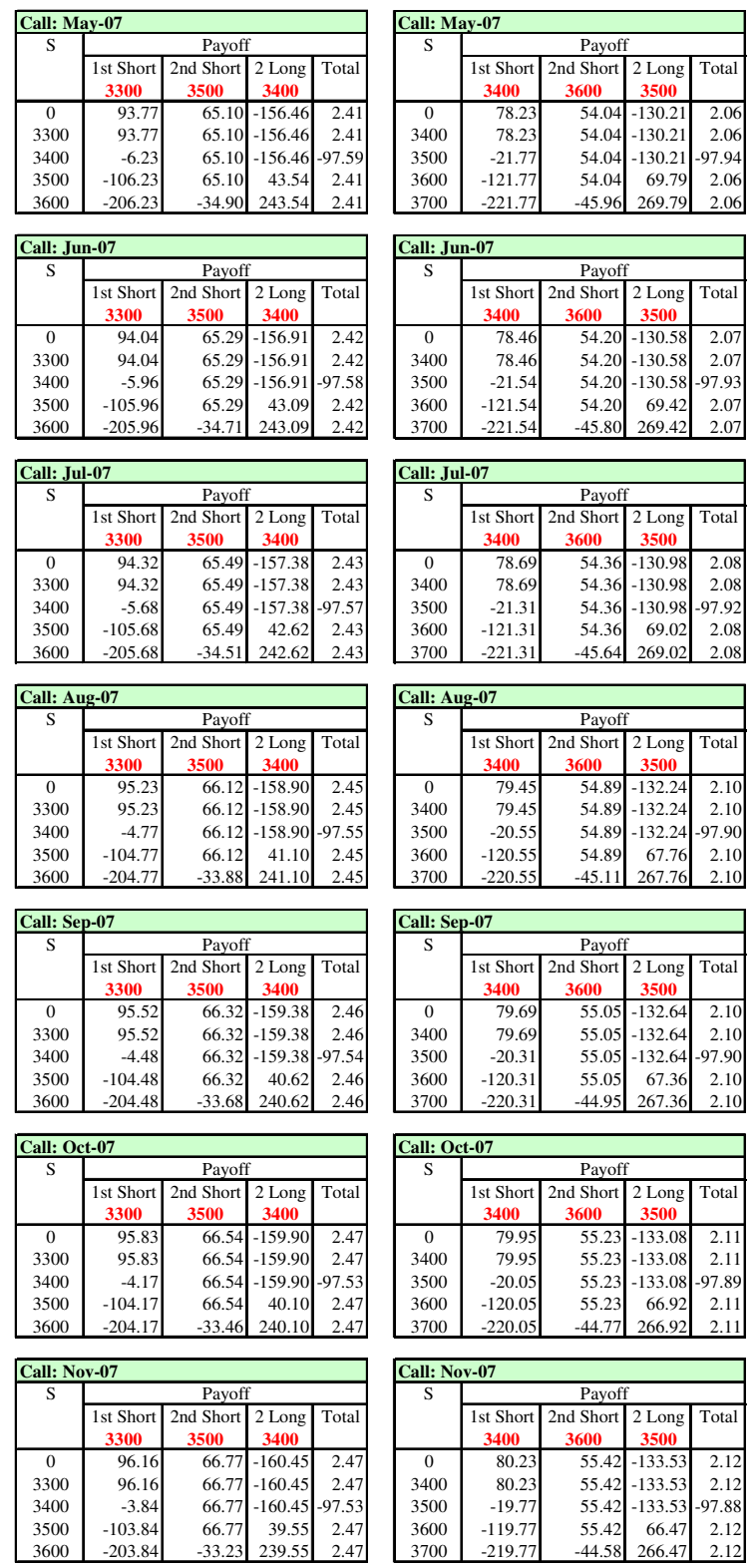

\begin{tabular}{|c|c|c|c|c|}
\hline \multicolumn{5}{|c|}{ Call: Dec-07 } \\
\hline \multirow[t]{2}{*}{$\mathrm{S}$} & \multicolumn{4}{|c|}{ Payoff } \\
\hline & $\begin{array}{c}\text { 1st Short } \\
3300\end{array}$ & $\begin{array}{c}\text { 2nd Short } \\
3500\end{array}$ & \begin{tabular}{|c|}
2 Long \\
3400
\end{tabular} & Total \\
\hline 0 & 96.47 & 66.98 & -160.96 & 2.48 \\
\hline 3300 & 96.47 & 66.98 & -160.96 & 2.48 \\
\hline 3400 & -3.53 & 66.98 & -160.96 & -97.52 \\
\hline 3500 & -103.53 & 66.98 & 39.04 & 2.48 \\
\hline 3600 & -203.53 & -33.02 & 239.04 & 2.48 \\
\hline
\end{tabular}




\section{Butterfly Spreads Using Put Options:}

If a significant movement in the copper price is expected, butterfly spreads can also be created by taking short positions on put options with the lowest and highest strike prices and taking two long positions on a put option with the middle strike price. All options have the same maturity date.

Table (7.42) demonstrates all possible butterfly spread strategies using put options for 2006 and 2007 based on the indications of 22 February 2005. There are 4 different spreads each month, so there are 96 butterfly spread strategies developed using put options for 24 months between Jan-06 and Dec-07.

As seen from table (7.42), all 48 spreads in 2006 would be profitable, but the most efficient spreads are created if the middle strike prices equal $\$ 2,600 / t$ and $\$ 2,400$. In 2007, creating butterfly spreads using January put options would not be efficient if the middle strike price is $\$ 2,300$. Hence, 84 butterfly spreads out of 96 could be useful in 2006 and 2007. The following tables construct the most efficient 3 spreads each year in three different cases including the middle strike prices are $\$ 2,400, \$ 2,500$, and $\$ 2,600$.

\section{Butterfly Spread for December 2006 (maximum profit)}

- Spot Price (22 Feb-05):

- Asian Put Options:

- Positions:
$\$ 3,367$

December-2006

Short Put@2300:

Short Put@2500:

Long 2 Puts@2400: premium $\$ 77.17$

premium $\$ 130.99$

premium $\$ 99.35$

Table 7.36: Profit from a Butterfly Spread (Dec-06 Put options, X: 2300/2400/2500)

\begin{tabular}{ccccc}
\hline $\begin{array}{c}\text { Copper price } \\
\text { Range }\end{array}$ & $\begin{array}{c}\text { Profit from } 1^{\text {st }} \\
\text { short put } \\
\text { @ } 2300\end{array}$ & $\begin{array}{c}\text { Profit from } 2^{\text {nd }} \\
\text { short put } \\
\text { @ } 2500\end{array}$ & $\begin{array}{c}\text { Profit from } 2 \\
\text { long puts } \\
\text { @ 2400 }\end{array}$ & $\begin{array}{c}\text { Total } \\
\text { profit }\end{array}$ \\
\hline $2300>\mathrm{S}_{\mathrm{AVG}}$ & $\mathrm{S}_{\mathrm{AVG}}-2217.42$ & $\mathrm{~S}_{\mathrm{AVG}}-2359.82$ & $4587.36-2 \mathrm{~S}_{\mathrm{AVG}}$ & 10.12 \\
$2400>\mathrm{S}_{\mathrm{AVG}}>2300$ & 82.58 & $\mathrm{~S}_{\mathrm{AVG}}-2359.82$ & $4587.36-2 \mathrm{~S}_{\mathrm{AVG}}$ & $2310.12-\mathrm{S}_{\mathrm{AVG}}$ \\
$2500>\mathrm{S}_{\mathrm{AVG}}>2400$ & 82.58 & $\mathrm{~S}_{\mathrm{AVG}}-2359.82$ & -212.64 & $\mathrm{~S}_{\mathrm{AVG}}-2489.88$ \\
$\mathrm{~S}_{\mathrm{AVG}}>2500$ & 82.58 & 140.18 & -212.64 & 10.12 \\
\hline
\end{tabular}


Profit is made when $S_{A V G(D e c-2006)}$ is less than $\$ 2,310.12$ or more than $\$ 2,489.88$ and is maximum of 10.12 when $\mathrm{S}_{\mathrm{AVG}(\mathrm{Dec}-2006)}$ is $\$ 2,300$ and less or $\$ 2,500$ and more.

\section{Butterfly Spread for December 2006 (maximum profit)}

- Spot Price (22 Feb-05):

- Asian Put Options:

- Positions:

\section{$\$ 3,367$}

December-2006

Short Put @ 2400:

Short Put@ 2600:

Long 2 Puts@ 2500: premium $\$ 99.35$

premium $\$ 162.76$

premium $\$ 130.99$

Table 7.37: Profit from a Butterfly Spread (Dec-06 Put options, X: 2400/2500/2600)

\begin{tabular}{ccccc}
\hline $\begin{array}{c}\text { Copper price } \\
\text { Range }\end{array}$ & $\begin{array}{c}\text { Profit from } 1^{\text {st }} \\
\text { short put } \\
\text { @ } 2400\end{array}$ & $\begin{array}{c}\text { Profit from } 2^{\text {nd }} \\
\text { short put } \\
\text { @ 2600 }\end{array}$ & $\begin{array}{c}\text { Profit from 2 } \\
\text { long puts } \\
\text { @ 2500 }\end{array}$ & $\begin{array}{c}\text { Total } \\
\text { profit }\end{array}$ \\
\hline $2400>\mathrm{S}_{\mathrm{AVG}}$ & $\mathrm{S}_{\mathrm{AVG}}-2293.68$ & $\mathrm{~S}_{\mathrm{AVG}}-2425.82$ & $4719.64-2 \mathrm{~S}_{\mathrm{AVG}}$ & 0.14 \\
$2500>\mathrm{S}_{\mathrm{AVG}}>2400$ & 106.32 & $\mathrm{~S}_{\mathrm{AVG}}-2425.82$ & $4719.64-2 \mathrm{~S}_{\mathrm{AVG}}$ & $2400.14-\mathrm{S}_{\mathrm{AVG}}$ \\
$2600>\mathrm{S}_{\mathrm{AVG}}>2500$ & 106.32 & $\mathrm{~S}_{\mathrm{AVG}}-2425.82$ & -280.36 & $\mathrm{~S}_{\mathrm{AVG}}-2599.86$ \\
$\mathrm{~S}_{\mathrm{AVG}}>2600$ & 106.32 & 174.18 & -280.36 & 0.14 \\
\hline
\end{tabular}

Profit is made when $S_{\mathrm{AVG}(\text { Dec-2006) }}$ is less than $\$ 2,400.14$ or more than $\$ 2,599.86$ and is maximum of 0.14 when $S_{\mathrm{AVG}(\operatorname{Dec}-2006)}$ is $\$ 2,400$ and less or $\$ 2,600$ and more.

\section{Butterfly Spread for December 2006 (maximum profit)}

- Spot Price (22 Feb-05):

- Asian Put Options:

- Positions:
$\$ 3,367$

December-2006

Short Put @ 2500: $\quad$ premium $\$ 130.99$

Short Put @ 2700: $\quad$ premium \$205.42

Long 2 Puts @ 2600: premium $\$ 162.76$

Table 7.38: Profit from a Butterfly Spread (Dec-06 Put options, X: 2500/2600/2700)

\begin{tabular}{ccccc}
\hline $\begin{array}{c}\text { Copper price } \\
\text { Range }\end{array}$ & $\begin{array}{c}\text { Profit from } 1^{\text {st }} \\
\text { short put } \\
\text { @ } 2500\end{array}$ & $\begin{array}{c}\text { Profit from } 2^{\text {nd }} \\
\text { short put } \\
\text { @ } 2700\end{array}$ & $\begin{array}{c}\text { Profit from } 2 \\
\text { long puts } \\
\text { @ 2600 }\end{array}$ & $\begin{array}{c}\text { Total } \\
\text { profit }\end{array}$ \\
\hline $2500>\mathrm{S}_{\mathrm{AVG}}$ & $\mathrm{S}_{\mathrm{AVG}}-2359.82$ & $\mathrm{~S}_{\mathrm{AVG}}-2480.17$ & $4851.64-2 \mathrm{~S}_{\mathrm{AVG}}$ & 11.65 \\
$2600>\mathrm{S}_{\mathrm{AVG}}>2500$ & 140.18 & $\mathrm{~S}_{\mathrm{AVG}}-2480.17$ & $4851.64-2 \mathrm{~S}_{\mathrm{AVG}}$ & $2511.65-\mathrm{S}_{\mathrm{AVG}}$ \\
$2700>\mathrm{S}_{\mathrm{AVG}}>2600$ & 140.18 & $\mathrm{~S}_{\mathrm{AVG}}-2480.17$ & -348.36 & $\mathrm{~S}_{\mathrm{AVG}}-2688.35$ \\
$\mathrm{~S}_{\mathrm{AVG}}>2700$ & 140.18 & 219.83 & -348.36 & 11.65 \\
\hline
\end{tabular}

Profit is made when $S_{A V G(D e c-2006)}$ is less than $\$ 2,511.65$ or more than $\$ 2,688.35$ and is maximum of 11.65 when $S_{\mathrm{AVG}(\mathrm{Dec}-2006)}$ is $\$ 2,500$ and less or $\$ 2,700$ and more. 


\section{Butterfly Spread for December 2007 (maximum profit)}

- Spot Price (22 Feb-05):

- Asian Put Options:

- Positions:
$\$ 3,367$

December-2007

Short Put @ 2300: $\quad$ premium $\$ 132.34$

Short Put @ 2500: $\quad$ premium $\$ 203.75$

Long 2 Puts @ 2400: $\quad$ premium \$162.34

Table 7.39: Profit from a Butterfly Spread (Dec-07 Put options, X: 2300/2400/2500)

\begin{tabular}{ccccc}
\hline $\begin{array}{c}\text { Copper price } \\
\text { Range }\end{array}$ & $\begin{array}{c}\text { Profit from } 1^{\text {st }} \\
\text { short put } \\
\text { @ 2300 }\end{array}$ & $\begin{array}{c}\text { Profit from } 2^{\text {nd }} \\
\text { short put } \\
\text { @ 2500 }\end{array}$ & $\begin{array}{c}\text { Profit from 2 } \\
\text { long puts } \\
\text { @ 2400 }\end{array}$ & $\begin{array}{c}\text { Total } \\
\text { profit }\end{array}$ \\
\hline $2300>\mathrm{S}_{\mathrm{AVG}}$ & $\mathrm{S}_{\mathrm{AVG}}-2152.07$ & $\mathrm{~S}_{\mathrm{AVG}}-2272.25$ & $4437.07-2 \mathrm{~S}_{\mathrm{AVG}}$ & 12.75 \\
$2400>\mathrm{S}_{\mathrm{AVG}}>2300$ & 147.93 & $\mathrm{~S}_{\mathrm{AVG}}-2272.25$ & $4437.07-22 \mathrm{~S}_{\mathrm{AVG}}$ & $2312.75-\mathrm{S}_{\mathrm{AVG}}$ \\
$2500>\mathrm{S}_{\mathrm{AVG}}>2400$ & 147.93 & $\mathrm{~S}_{\mathrm{AVG}}-2272.25$ & -362.93 & $\mathrm{~S}_{\mathrm{AVG}}-2487.25$ \\
$\mathrm{~S}_{\mathrm{AVG}}>2500$ & 147.93 & 227.75 & -362.93 & 12.75 \\
\hline
\end{tabular}

Profit is made when $\mathrm{S}_{\mathrm{AVG}(\mathrm{Dec}-2007)}$ is less than $\$ 2,312.75$ or more than $\$ 2,487.25$ and is maximum of 12.75 when $S_{\mathrm{AVG}(\operatorname{Dec}-2007)}$ is $\$ 2,300$ and less or $\$ 2,500$ and more.

\section{Butterfly Spread for December 2007 (maximum profit)}

- Spot Price (22 Feb-05):

- Asian Put Options:

- Positions:
$\$ 3,367$

December-2007

Short Put@2400:

Short Put@2600:

Long 2 Puts@2500: premium $\$ 162.34$

premium $\$ 250.43$

premium $\$ 203.75$

Table 7.40: Profit from a Butterfly Spread (Dec-07 Put options, X: 2400/2500/2600)

\begin{tabular}{ccccc}
\hline $\begin{array}{c}\text { Copper price } \\
\text { Range }\end{array}$ & $\begin{array}{c}\text { Profit from } 1^{\text {st }} \\
\text { short put } \\
\text { @ } 2400\end{array}$ & $\begin{array}{c}\text { Profit from } 2^{\text {nd }} \\
\text { short put } \\
\text { @ } 2600\end{array}$ & $\begin{array}{c}\text { Profit from } 2 \\
\text { long puts } \\
\text { @ 2500 }\end{array}$ & $\begin{array}{c}\text { Total } \\
\text { profit }\end{array}$ \\
\hline $2400>\mathrm{S}_{\mathrm{AVG}}$ & $\mathrm{S}_{\mathrm{AVG}}-2218.53$ & $\mathrm{~S}_{\mathrm{AVG}}-2320.07$ & $4544.49-2 \mathrm{~S}_{\mathrm{AVG}}$ & 5.89 \\
$2500>\mathrm{S}_{\mathrm{AVG}}>2400$ & 181.47 & $\mathrm{~S}_{\mathrm{AVG}}-2320.07$ & $4544.49-2 \mathrm{~S}_{\mathrm{AVG}}$ & $2405.89-\mathrm{S}_{\mathrm{AVG}}$ \\
$2600>\mathrm{S}_{\mathrm{AVG}}>2500$ & 181.47 & $\mathrm{~S}_{\mathrm{AVG}}-2320.07$ & -455.51 & $\mathrm{~S}_{\mathrm{AVG}}-2594.11$ \\
$\mathrm{~S}_{\mathrm{AVG}}>2600$ & 181.47 & 279.93 & -455.51 & 5.89 \\
\hline
\end{tabular}

Profit is made when $\mathrm{S}_{\mathrm{AVG}(\mathrm{Dec}-2007)}$ is less than $\$ 2,405.89$ or more than $\$ 2,594.11$ and is maximum of 5.89 when $S_{\mathrm{AVG}(\text { Dec-2007) }}$ is $\$ 2,400$ and less or $\$ 2,600$ and more. 


\section{Butterfly Spread for December 2007 (maximum profit)}

- Spot Price (22 Feb-05):

- Asian Put Options:

- Positions:
$\$ 3,367$

December-2007

Short Put@ 2500:

Short Put@2700:

Long 2 Puts@2600: premium $\$ 203.75$

premium $\$ 302.15$

premium $\$ 250.43$

Table 7.41: Profit from a Butterfly Spread (Dec-07 Put options, X: 2500/2600/2700)

\begin{tabular}{ccccc}
\hline $\begin{array}{c}\text { Copper price } \\
\text { Range }\end{array}$ & $\begin{array}{c}\text { Profit from } 1^{\text {st }} \\
\text { short put } \\
\text { @ } 2500\end{array}$ & $\begin{array}{c}\text { Profit from } 2^{\text {nd }} \\
\text { short put } \\
\text { @ 2700 }\end{array}$ & $\begin{array}{c}\text { Profit from 2 } \\
\text { long puts } \\
\text { @ 2600 }\end{array}$ & $\begin{array}{c}\text { Total } \\
\text { profit }\end{array}$ \\
\hline $2500>\mathrm{S}_{\mathrm{AVG}}$ & $\mathrm{S}_{\mathrm{AVG}}-2272.25$ & $\mathrm{~S}_{\mathrm{AVG}}-2362.25$ & $4640.13-2 \mathrm{~S}_{\mathrm{AVG}}$ & 5.63 \\
$2600>\mathrm{S}_{\mathrm{AVG}}>2500$ & 227.75 & $\mathrm{~S}_{\mathrm{AVG}}-2362.25$ & $4640.13-2 \mathrm{~S}_{\mathrm{AVG}}$ & $2505.63-\mathrm{S}_{\mathrm{AVG}}$ \\
$2700>\mathrm{S}_{\mathrm{AVG}}>2600$ & 227.75 & $\mathrm{~S}_{\mathrm{AVG}}-2362.25$ & -559.87 & $\mathrm{~S}_{\mathrm{AVG}}-2694.37$ \\
$\mathrm{~S}_{\mathrm{AVG}}>2700$ & 227.75 & 337.75 & -559.87 & 5.63 \\
\hline
\end{tabular}

Profit is made when $S_{\mathrm{AVG}(\text { Dec-2007) }}$ is less than $\$ 2,505.63$ or more than $\$ 2,694.37$ and is maximum of 5.63 when $\mathrm{S}_{\mathrm{AVG}(\mathrm{Dec}-2007)}$ is $\$ 2,500$ and less or $\$ 2,700$ and more.

Figure (7.30): Butterfly Spread, Put Options Dec-06, X: 2300/2400/2500

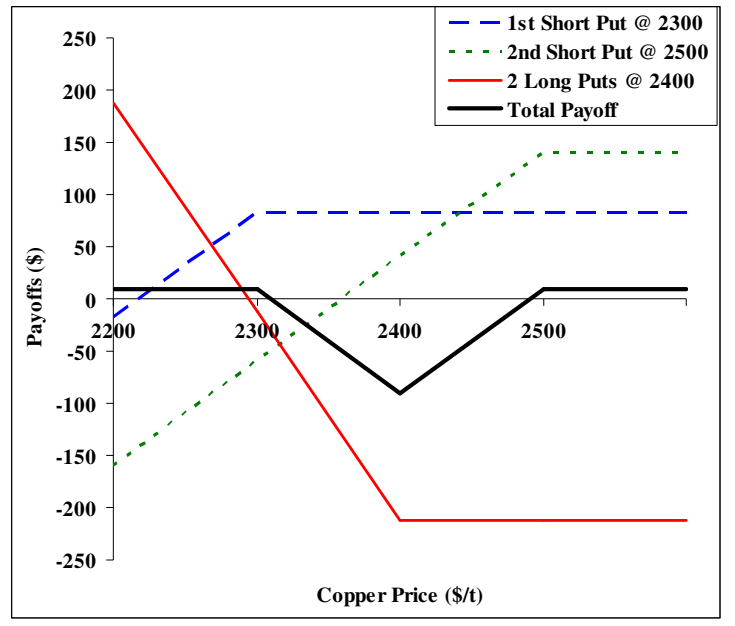

Figure (7.31): Butterfly Spread, Put Options Dec-06, X: 2400/2500/2600

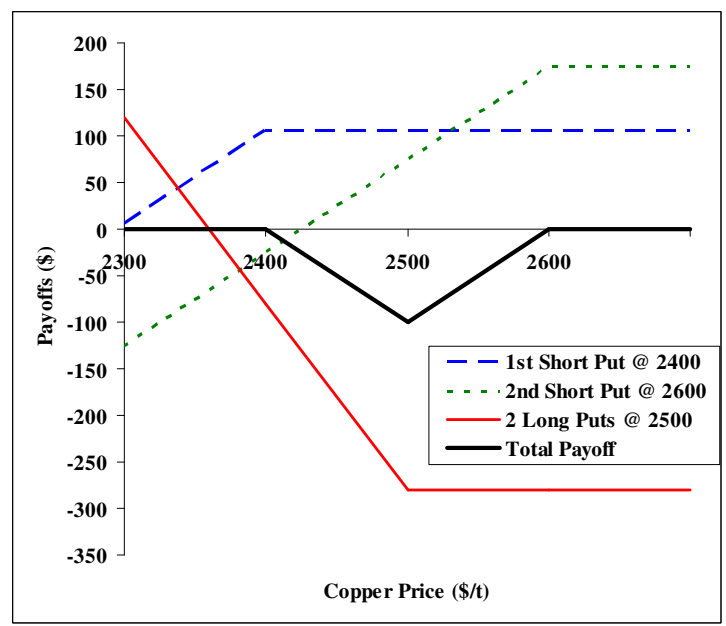


Figure (7.32): Butterfly Spread, Put Options Dec-06, X: 2500/2600/2700

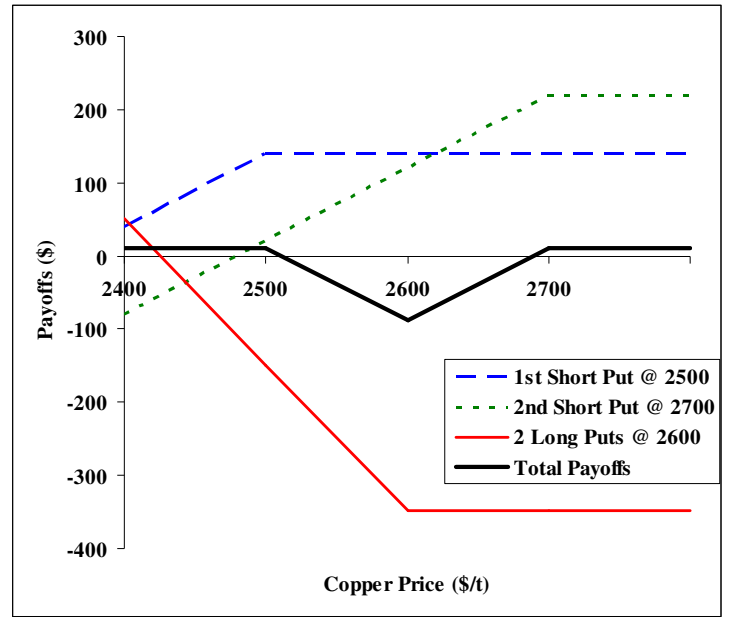

Figure (7.33): Butterfly Spread, Put Options Dec-07, X: 2300/2400/2500

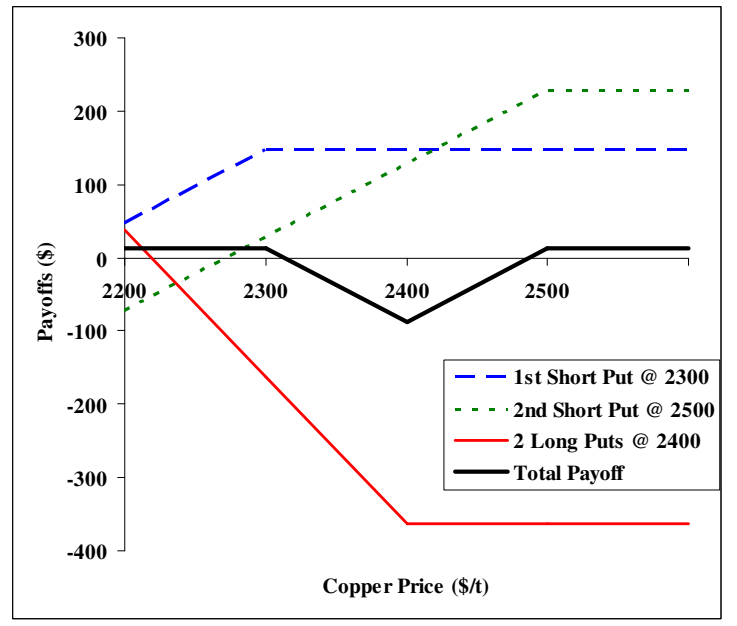

Figure (7.34): Butterfly Spread, Put Options

Dec-07, X: 2400/2500/2600

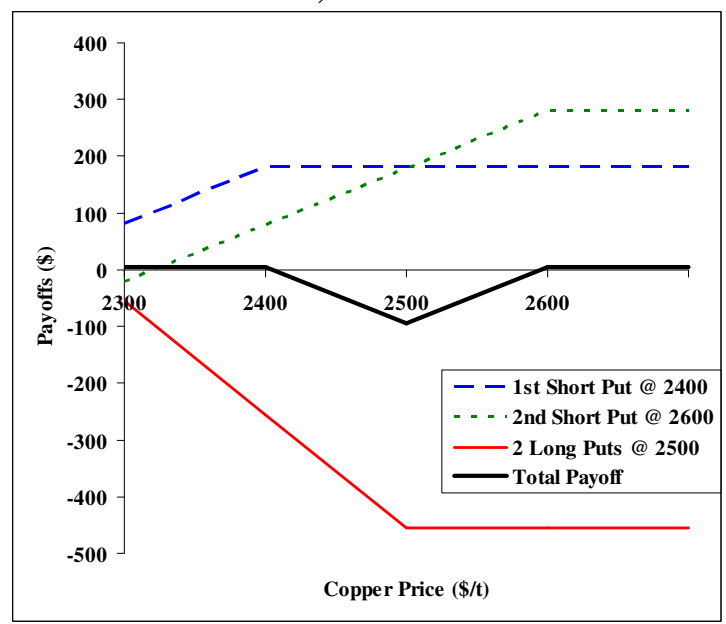

Figure (7.35): Butterfly Spread, Put Options Dec-07, X: 2500/2600/2700

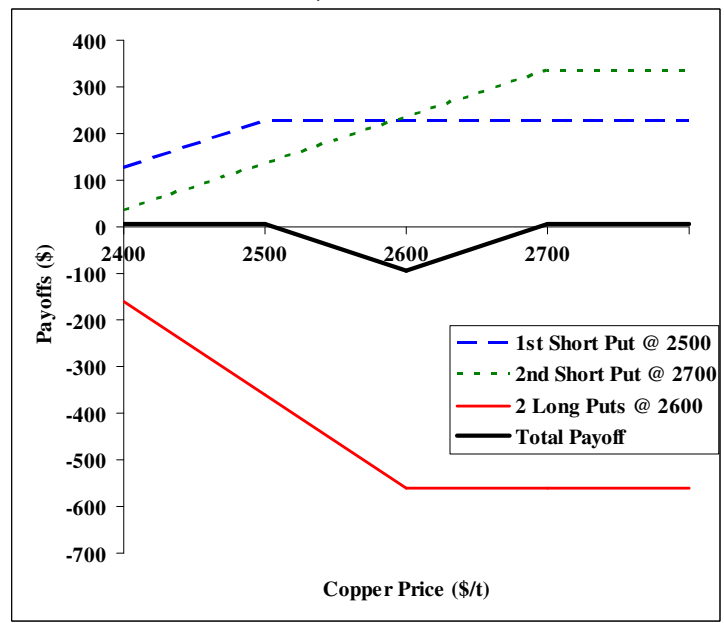

Comparing the 84 butterfly spreads, which were created using put options in 2006 and 2007, the spreads on Dec-06 and Dec-07 puts were more effective because if the price fluctuates in either direction from the middle strike prices, the profit would be greatest for Dec-06 and Dec-07 spreads. Moreover, the loss would be no more than $\$ 89.88, \$ 99.86$, and $\$ 88.35$ for Dec-2006 spreads and $\$ 87.25, \$ 94.11$, and $\$ 94.37$ for Dec-2007 spreads if the price settles around the middle strike prices. 
Table 7.42: Butterfly Spreads Using Put Options for 2006 and 2007
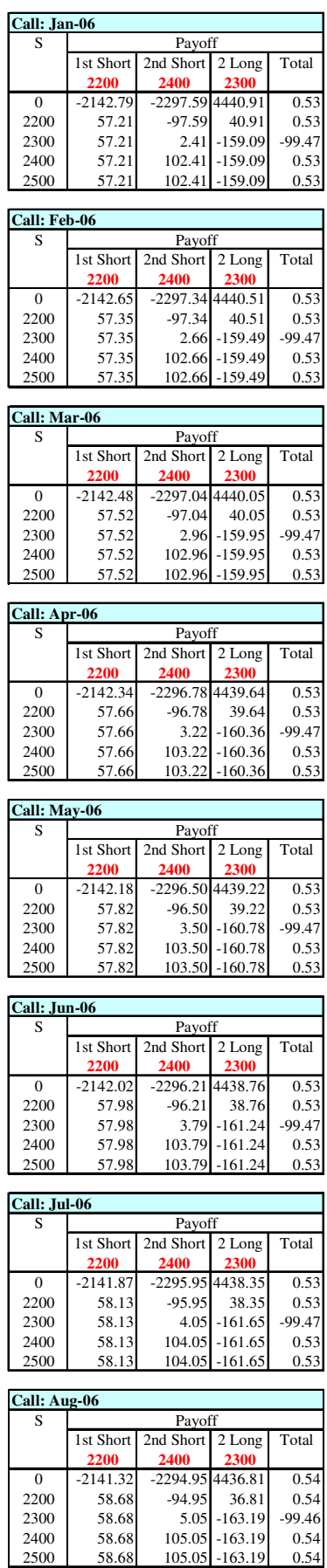
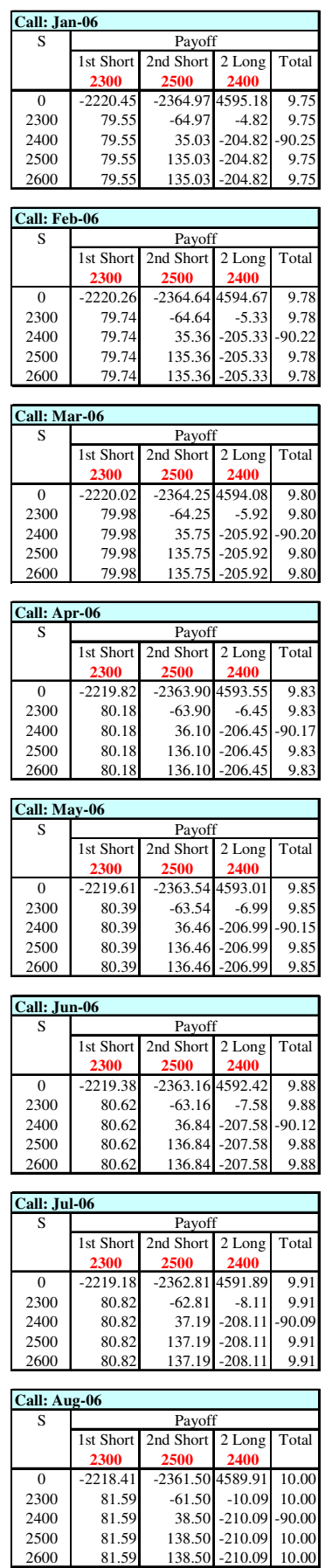
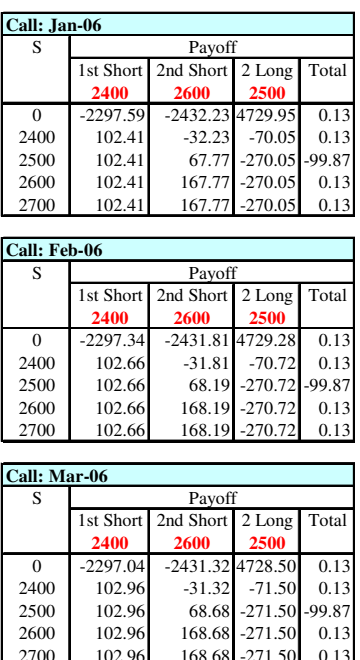

\begin{tabular}{|c|c|c|c|}
\hline & -271.50 & 0.13 \\
\hline
\end{tabular}
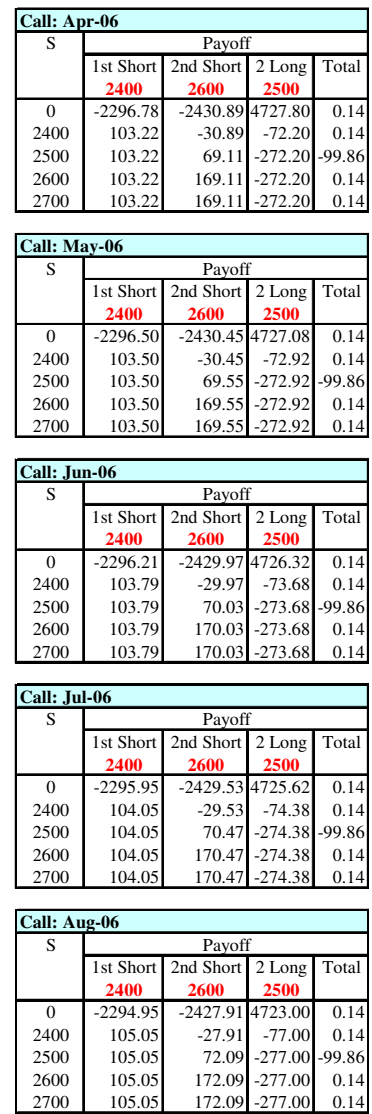
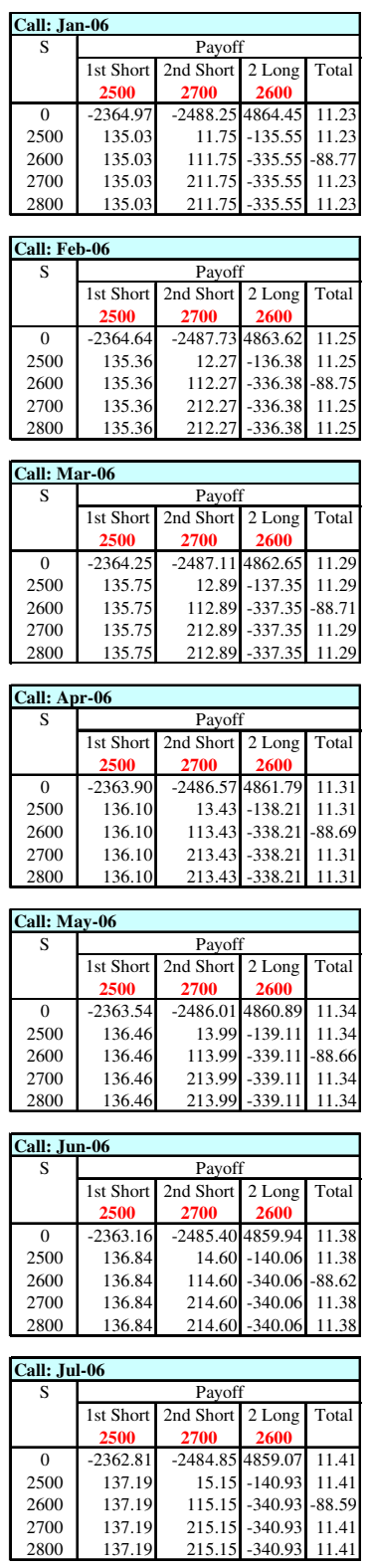

\begin{tabular}{|c|r|r|r|r|}
\hline \multicolumn{5}{|c|}{ Call: Aug-06 } \\
\hline S & \multicolumn{4}{|c|}{ Payoff } \\
\cline { 2 - 5 } & \multicolumn{1}{|c|}{$\mathbf{2 5 0 0}$} & \multicolumn{1}{|c|}{$\mathbf{2 7 0 0}$} & $\mathbf{2 6 0 0}$ & \\
\hline 0 & -2361.50 & -2482.80 & 4855.82 & 11.51 \\
2500 & 138.50 & 17.20 & -144.18 & 11.51 \\
2600 & 138.50 & 117.20 & -344.18 & -88.49 \\
2700 & 138.50 & 217.20 & -344.18 & 11.51 \\
2800 & 138.50 & 217.20 & -344.18 & 11.51 \\
\hline
\end{tabular}


Table 7.42 continued
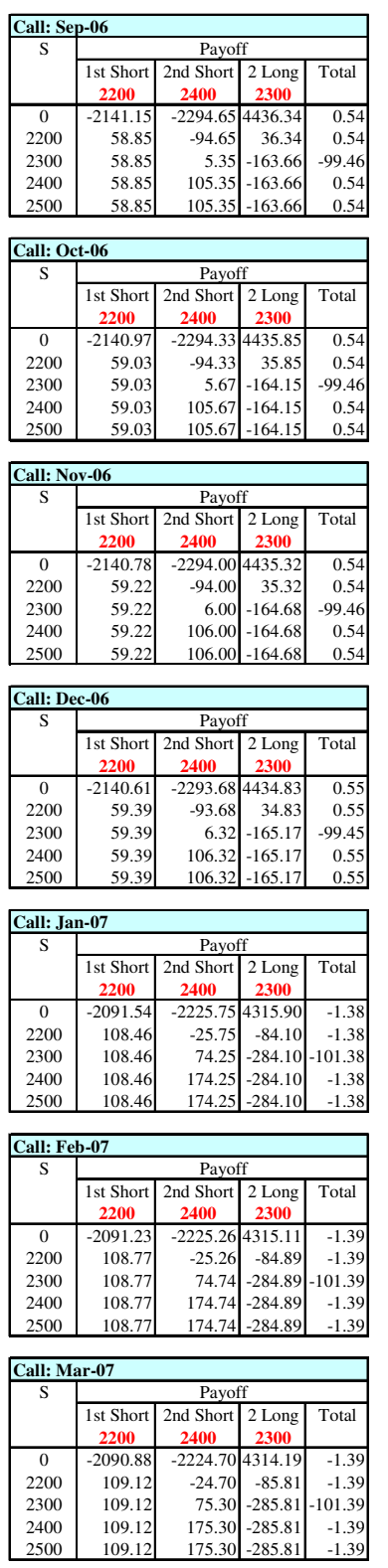

\begin{tabular}{|c|r|r|r|r|}
\hline \multicolumn{4}{|l|}{ Call: Apr-07 } \\
\hline \multirow{2}{*}{$\mathrm{S}$} & \multicolumn{4}{|c|}{ Payoff } \\
\cline { 2 - 5 } & \multicolumn{1}{|c|}{ st Short } & 2nd Short & 2 Long & \multicolumn{1}{|c|}{ Total } \\
& $\mathbf{2 4 0 0}$ & $\mathbf{2 3 0 0}$ & \\
\hline 0 & -2090.57 & -2224.20 & 4313.37 & -1.40 \\
2200 & 109.43 & -24.20 & -86.63 & -1.40 \\
2300 & 109.43 & 75.80 & -286.63 & -101.40 \\
2400 & 109.43 & 175.80 & -286.63 & -1.40 \\
2500 & 109.43 & 175.80 & -286.63 & -1.40 \\
\hline
\end{tabular}
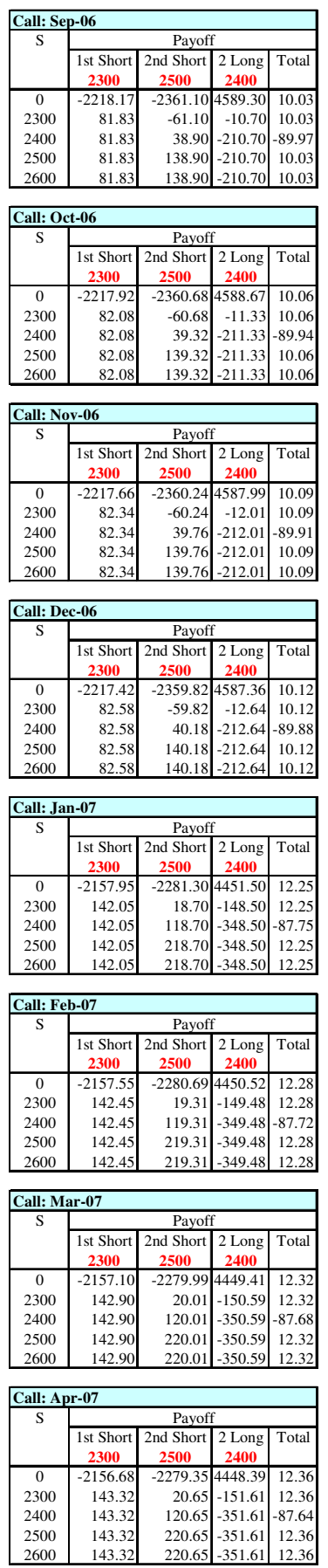
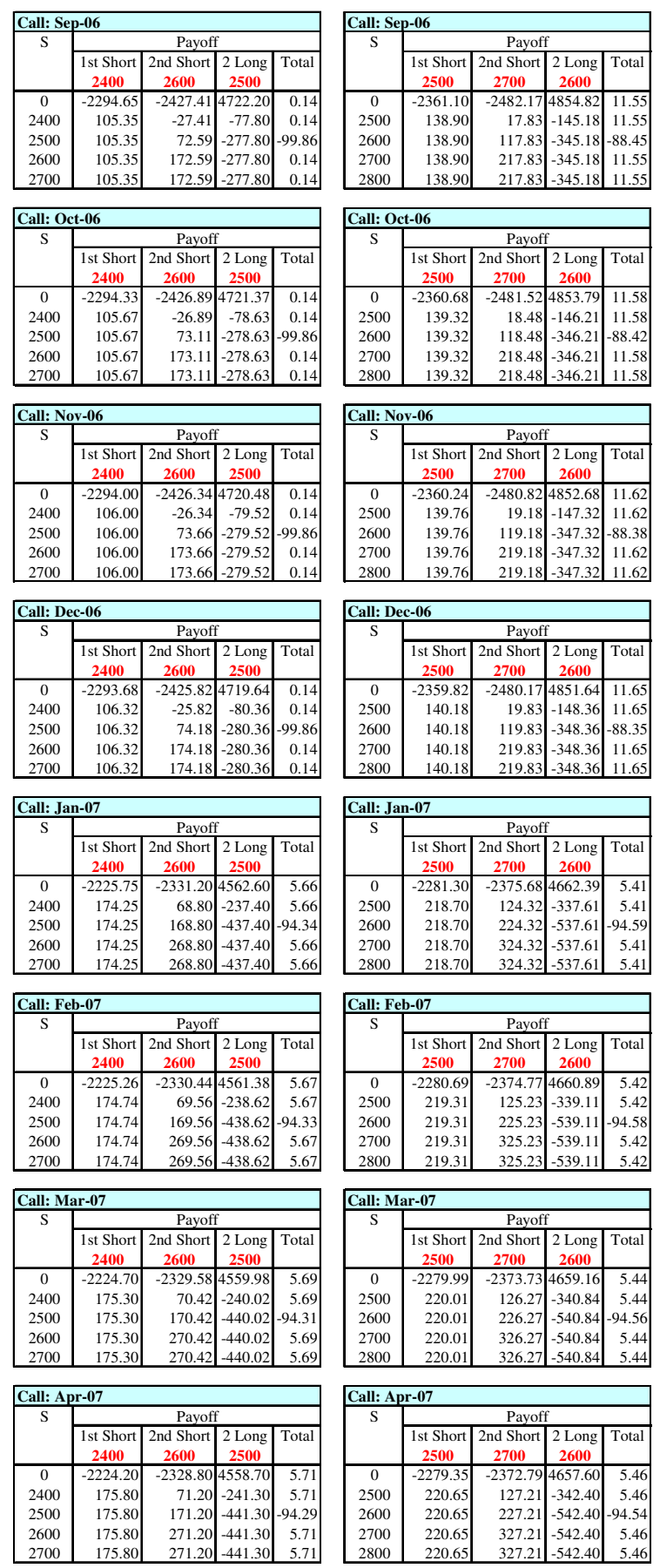
Table 7.42 continued
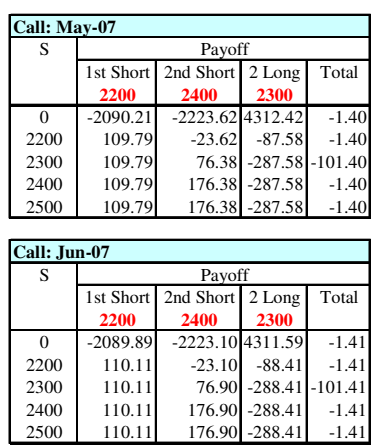

\begin{tabular}{|c|c|c|c|c|}
\hline \multicolumn{5}{|c|}{ all: Jul-07 } \\
\hline \multirow[t]{2}{*}{$\mathrm{S}$} & \multicolumn{4}{|c|}{ Payoff } \\
\hline & 1st Short & 2nd Short & 2 Long & Total \\
\hline 0 & -2089.56 & -2222.57 & 4310.73 & -1.41 \\
\hline 2200 & 110.44 & -22.57 & -89.27 & -1.41 \\
\hline 2300 & 110.44 & 77.43 & -289.27 & -101.41 \\
\hline 2400 & 110.44 & 177.43 & -289.27 & -1.41 \\
\hline 2500 & 110.44 & 177.43 & 289. & -1.41 \\
\hline
\end{tabular}

\begin{tabular}{|c|r|r|r|r|}
\hline \multicolumn{5}{|c|}{ Call: Aug-07 } \\
\hline $\mathrm{S}$ & \multicolumn{4}{|c|}{ Payoff } \\
\cline { 2 - 5 } & \multicolumn{1}{|c|}{ 1st Short } & 2nd Short & 2 Long & \multicolumn{1}{c|}{ Total } \\
& \multicolumn{2}{|c|}{$\mathbf{2 4 0 0}$} & $\mathbf{2 3 0 0}$ & \\
\hline 0 & -2088.49 & -2220.86 & 4307.93 & -1.42 \\
2200 & 111.51 & -20.86 & -92.07 & -1.42 \\
2300 & 111.51 & 79.14 & -292.07 & -101.42 \\
2400 & 111.51 & 179.14 & -292.07 & -1.42 \\
2500 & 111.51 & 179.14 & -292.07 & -1.42 \\
\hline
\end{tabular}

\begin{tabular}{|c|r|r|r|r|}
\hline \multicolumn{5}{|c|}{ Call: Sep-07 } \\
\hline $\mathrm{S}$ & \multicolumn{4}{|c|}{ Payoff } \\
\cline { 2 - 5 } & \multicolumn{1}{|c|}{$\mathbf{2 2 0 0}$} & \multicolumn{1}{|c|}{$\mathbf{2 4 0 0}$} & $\mathbf{2 3 0 0}$ & \multicolumn{1}{|c|}{ Total } \\
\hline 0 & -2088.16 & -2220.32 & $\mathbf{4 3 0 7 . 0 6}$ & -1.43 \\
2200 & 111.84 & -20.32 & -92.94 & -1.43 \\
2300 & 111.84 & 79.68 & -292.94 & -101.43 \\
2400 & 111.84 & 179.68 & -292.94 & -1.43 \\
2500 & 111.84 & 179.68 & -292.94 & -1.43 \\
\hline
\end{tabular}

\begin{tabular}{|c|c|c|c|c|}
\hline \multicolumn{5}{|c|}{\begin{tabular}{|l|} 
Call: Oct-07 \\
\end{tabular}} \\
\hline \multirow{2}{*}{ 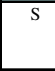 } & \multicolumn{4}{|c|}{ Payoff } \\
\hline & $\begin{array}{c}1 \text { 1st Short } \\
\mathbf{2 2 0 0}\end{array}$ & $\begin{array}{c}\text { 2nd Short } \\
2400\end{array}$ & $\begin{array}{c}2 \text { Long } \\
2300\end{array}$ & \begin{tabular}{|l|l|} 
Total \\
\end{tabular} \\
\hline 0 & -2087.79 & -2219.73 & 4306.09 & -1.43 \\
\hline 2200 & 112.21 & -19.73 & -93.91 & -1.43 \\
\hline 2300 & 112.21 & 80.27 & -293.91 & -101.43 \\
\hline 2400 & 112.21 & 180.27 & -293.91 & -1.43 \\
\hline 2500 & 112 & 180.2 & & -1.43 \\
\hline
\end{tabular}

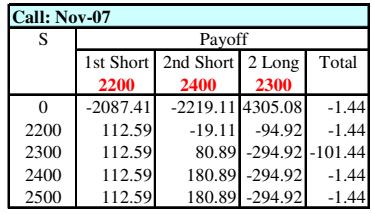

\begin{tabular}{|c|r|r|r|r|}
\hline \multicolumn{5}{|l|}{ Call: Dec-07 } \\
\hline \multirow{2}{*}{$\mathrm{S}$} & \multicolumn{4}{|c|}{ Payoff } \\
\cline { 2 - 5 } & \multicolumn{1}{|c|}{$\mathbf{2 2 0 0}$} & \multicolumn{1}{|c|}{$\mathbf{2 4 0 0}$} & $\mathbf{2 3 0 0}$ & \\
\hline 0 & -2087.05 & -2218.53 & 4304.14 & -1.44 \\
2200 & 112.95 & -18.53 & -95.86 & -1.44 \\
2300 & 112.95 & 81.47 & -295.86 & -101.44 \\
2400 & 112.95 & 181.47 & -295.86 & -1.44 \\
2500 & 112.95 & 181.47 & -295.86 & -1.44 \\
\hline
\end{tabular}
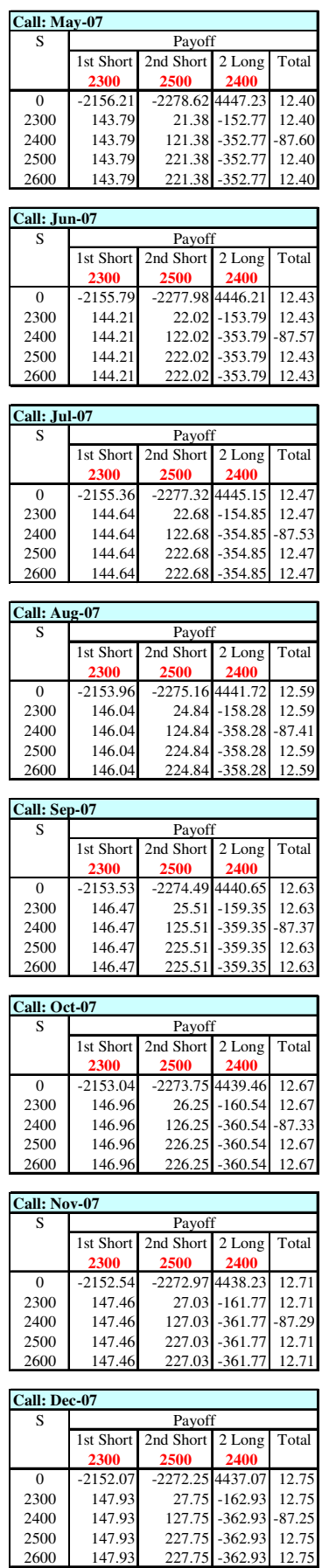
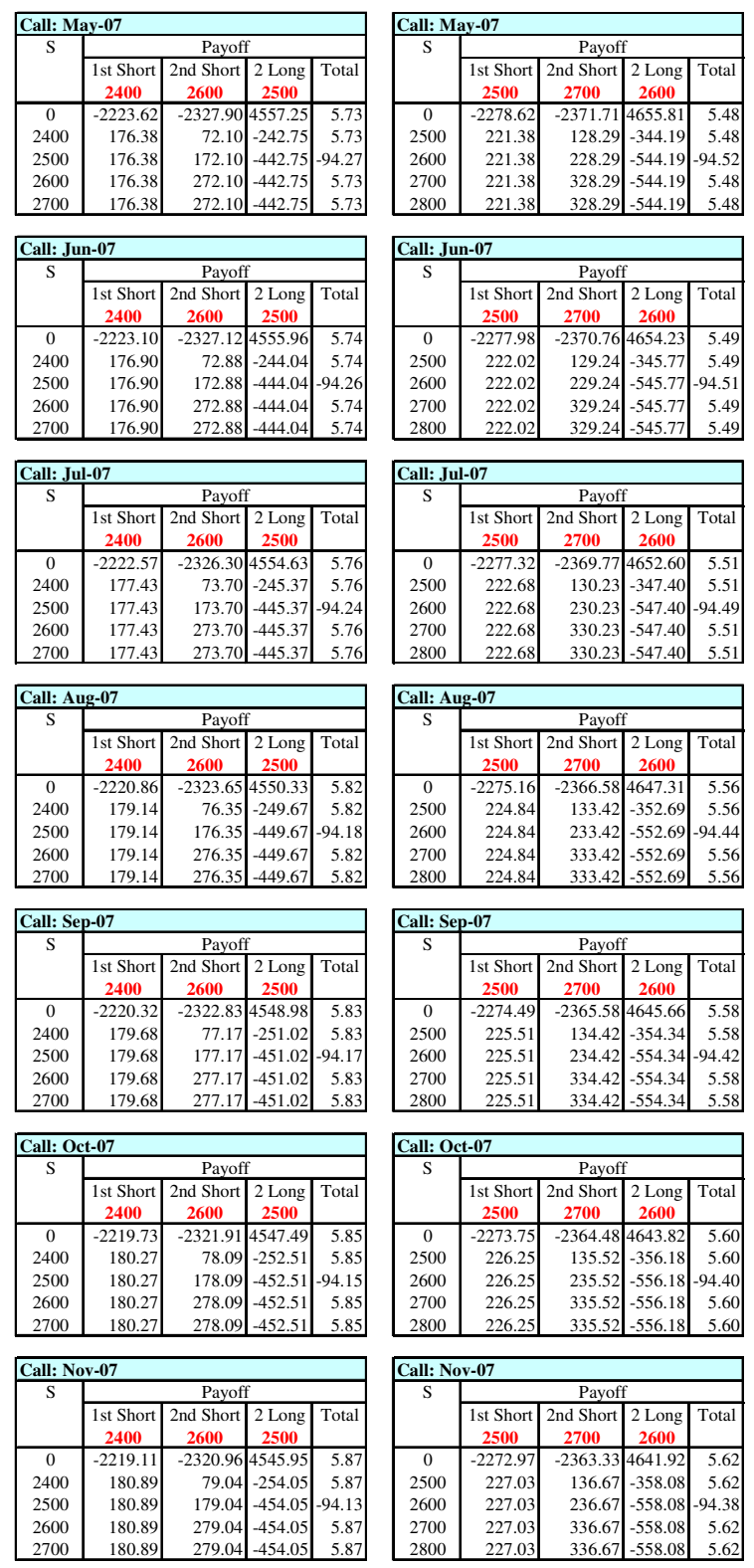

\begin{tabular}{|c|r|r|r|r|}
\hline \multicolumn{5}{|l|}{ Call: Dec-07 } \\
\hline S & \multicolumn{4}{|c|}{ Payoff } \\
\cline { 2 - 6 } & \multicolumn{1}{|c|}{ 1st Short } & 2nd Short & 2 Long & Total \\
& \multicolumn{2400}{|c|}{$\mathbf{2 6 0 0}$} & $\mathbf{2 5 0 0}$ & \\
\hline 0 & -2218.53 & -2320.07 & 4544.49 & 5.89 \\
2400 & 181.47 & 79.93 & -255.51 & 5.89 \\
2500 & 181.47 & 179.93 & -455.51 & -94.11 \\
2600 & 181.47 & 279.93 & -455.51 & 5.89 \\
2700 & 181.47 & 279.93 & -455.51 & 5.89 \\
\hline
\end{tabular}

\begin{tabular}{|c|r|r|r|r|}
\hline \multicolumn{5}{|c|}{ Call: Dec-07 } \\
\hline S & \multicolumn{4}{|c|}{ Payoff } \\
\cline { 2 - 5 } & \multicolumn{1}{|c|}{$\mathbf{2 5 0 0}$} & \multicolumn{1}{|c|}{$\mathbf{2 7 0 0}$} & $\mathbf{2 6 0 0}$ & Total \\
\hline 0 & -2272.25 & -2362.25 & 4640.13 & 5.63 \\
2500 & 227.75 & 137.75 & -359.87 & 5.63 \\
2600 & 227.75 & 237.75 & -559.87 & -94.37 \\
2700 & 227.75 & 337.75 & -559.87 & 5.63 \\
2800 & 227.75 & 337.75 & -559.87 & 5.63 \\
\hline
\end{tabular}


Three basic spreads involving option strategies have been analyzed using the monthly settled average price of call and put options until 2007. Combinations such as straddles, strips, straps and strangles are also useful option trading strategies. They involve taking a position in both call and put options on the underlying asset (Hull, 2002, p.211-214). Except for strangles, other combinations (straddles, strips and straps) are created by taking positions on a call and a put option with the same exercise price. The data quotes, which contain call and put options with the same strike price, were not available on 22 Feb-05, the day used in this analysis. Therefore, only strangles can be constructed using a call and a put option because an investor takes a long position on a call and a put with different strike prices and the same expiration date. However, it is appropriate to use strangles only if there is expected to be a large movement in prices. Hence, avoiding this risky strategy is better than expecting to bet that there will be a large price move that is uncertain whether it will increase or decrease. For example, let us consider a strangle strategy that is created by buying a put option at $\$ 2,700 / \mathrm{t}$ and buying a call option at $\$ 3,600 /$ for January 2007 options.

Strangle for January 2007 (minimum profit)

- Spot Price (22 Feb-05):

$\$ 3,367$

- Asian Call/Put Options:

January-2007

- Positions:

Long Call @ 3600: $\quad$ premium $\$ 49.74$

Long Put @ 2700: $\quad$ premium \$302.15

Table 7.43: Profit from a Strangle (Jan-07 Call/Put options, X: 3600/2700)

\begin{tabular}{cccc}
\hline $\begin{array}{c}\text { Copper price } \\
\text { range }\end{array}$ & $\begin{array}{c}\text { Profit from long } \\
\text { call @ 3600 }\end{array}$ & $\begin{array}{c}\text { Profit from long } \\
\text { put @ 2700 }\end{array}$ & $\begin{array}{c}\text { Total } \\
\text { profit }\end{array}$ \\
\hline $\mathrm{S}_{\mathrm{AVG}}>3600$ & $\mathrm{~S}_{\mathrm{AVG}}-3653.39$ & -324.32 & $\mathrm{~S}_{\mathrm{AVG}}-3977.71$ \\
$3600 \geq \mathrm{S}_{\mathrm{AVG}} \geq 2700$ & -53.39 & -324.32 & -327.71 \\
$\mathrm{~S}_{\mathrm{AVG}}<2700$ & -53.39 & $2375.68-\mathrm{S}_{\mathrm{AVG}}$ & $2322.29-\mathrm{S}_{\mathrm{AVG}}$ \\
\hline
\end{tabular}


In this example, profit is maid when $\mathrm{S}_{\mathrm{AVG}(\mathrm{Jan}-2007)}$ is less than $\$ 2,322.29$ or more than $\$ 3,977.71$. If the monthly average price of Jan-07 is between $\$ 3,600$ and $\$ 2,700$, the loss would be $\$ 327.71$. The figure below shows this payoff pattern.

Figure (7.36): Payoff Diagram of Strangle for Jan-07, X: 2700/3600

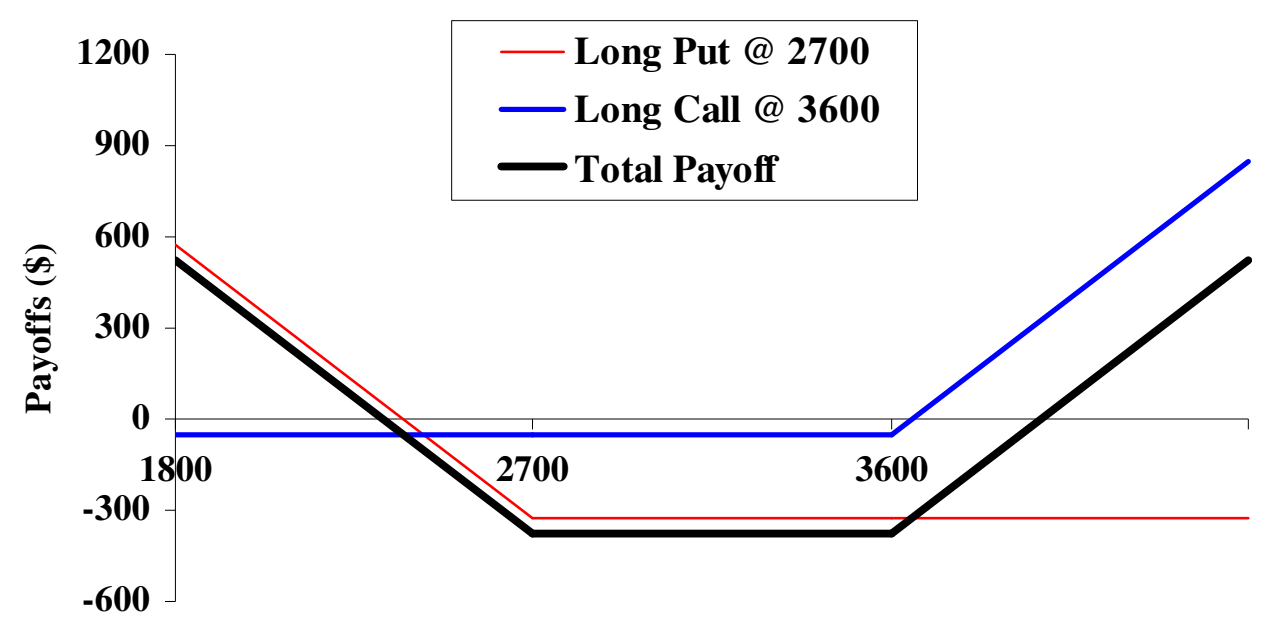

Copper Price $(\$ / t)$

\subsection{SINGLE POSITIONS IN OPTIONS}

In this part, single positions in call and put options will be evaluated based on 22 February 2005, the day of data.

\subsubsection{A PRODUCER'S STRATEGIES:}

A producer can buy a put option or sell a call option in order to insure against a fall in copper prices. If the producer takes a long position in a put option, he/she will pay the premium of the put option at time zero and receives the maximum of $\left(0, \mathrm{X}-\mathrm{S}_{\mathrm{AVG}}\right)$ at the option's maturity. If the producer takes a short position in a call option, he/she will 
receive the premium of the call option at time zero, but pays the maximum of $\left(\mathrm{X}-\mathrm{S}_{\mathrm{AVG}}\right.$, 0) at the option's maturity. Buying put options for 2006 and 2007 maturities was not optimal because the highest strike price was $\$ 2,700 / t$ on the day of data. Thus, taking a long position in put options would not be the useful strategy.

The following table demonstrates probabilities for short call option positions to be profitable based on the results in table (6.2).

Table 7.44: Probabilities for Written Call Options to be Profitable

\begin{tabular}{|c|c|c|c|c|}
\hline \multirow{3}{*}{ Year } & \multirow{2}{*}{$\begin{array}{c}\text { Strategy } \\
\text { Write a Call @ }\end{array}$} & \multicolumn{2}{|c|}{ Payoff $(\$)$} & Probability of \\
\cline { 2 - 4 } & 3100 & 159.99 & $3100-\mathrm{S}_{\mathrm{AVG}}$ & 0.30 \\
& 3200 & 133.05 & $3200-\mathrm{S}_{\mathrm{AVG}}$ & 0.42 \\
& 3300 & 110.09 & $3300-\mathrm{S}_{\mathrm{AVG}}$ & 0.55 \\
& 3400 & 90.67 & $3400-\mathrm{S}_{\mathrm{AVG}}$ & 0.66 \\
& 3500 & 74.35 & $3500-\mathrm{S}_{\mathrm{AVG}}$ & 0.76 \\
& 3600 & 60.72 & $3600-\mathrm{S}_{\mathrm{AVG}}$ & 0.83 \\
\hline \multirow{6}{*}{2007} & 3100 & 122.86 & $3100-\mathrm{S}_{\mathrm{AVG}}$ & 0.12 \\
& 3200 & 103.13 & $3200-\mathrm{S}_{\mathrm{AVG}}$ & 0.19 \\
& 3300 & 86.30 & $3300-\mathrm{S}_{\mathrm{AVG}}$ & 0.28 \\
& 3400 & 72.00 & $3400-\mathrm{S}_{\mathrm{AVG}}$ & 0.39 \\
& 3500 & 59.92 & $3500-\mathrm{S}_{\mathrm{AVG}}$ & 0.51 \\
& 3600 & 49.74 & $3600-\mathrm{S}_{\mathrm{AVG}}$ & 0.63 \\
\hline
\end{tabular}

As can be seen from this table, selling a call option at $\$ 3,500$ or $\$ 3,600$ is likely to be profitable and the rest of strategies may not be profitable. However, it may be optimal to producers not to implement risk management under the circumstance of a bullish trend in the copper market. The copper market seems to be bullish in 2006 and 2007; thus, copper producers may not suffer from a severe price risk during this period. 


\subsubsection{A CONSUMER'S STRATEGIES:}

Copper consumers can take a short position in a put option or a long position in a call option in order to protect against a rise in copper prices. Buying a call option would not be optimal because a copper consumer would pay a call premium initially and a payoff at the option's maturity may not fully cover this negative payout. Instead of buying a call option, selling a put option would be optimal to copper consumers.

Table 7.45: Probabilities for Written Put Options to be Profitable

\begin{tabular}{|c|c|c|c|c|}
\hline \multirow{3}{*}{ Year } & Strategy & \multicolumn{2}{|c|}{ Payoffs $(\$)$} & Probability of \\
\cline { 3 - 4 } & Write a Put @ & Time 0 & Time & being profitable \\
\cline { 2 - 4 } 2006 & 2200 & 55.50 & $\mathrm{~S}_{\mathrm{AVG}}-2200$ & 1.00 \\
& 2300 & 77.17 & $\mathrm{~S}_{\mathrm{AVG}}-2300$ & 1.00 \\
& 2400 & 99.35 & $\mathrm{~S}_{\mathrm{AVG}}-2400$ & 1.00 \\
& 2500 & 130.99 & $\mathrm{~S}_{\mathrm{AVG}}-2500$ & 1.00 \\
& 2600 & 162.76 & $\mathrm{~S}_{\mathrm{AVG}}-2600$ & 0.98 \\
& 2700 & 205.42 & $\mathrm{~S}_{\mathrm{AVG}}-2700$ & 0.97 \\
\hline \multirow{6}{*}{2007} & 2200 & 101.05 & $\mathrm{~S}_{\mathrm{AVG}}-2200$ & 1.00 \\
& 2300 & 132.34 & $\mathrm{~S}_{\mathrm{AVG}}-2300$ & 1.00 \\
& 2400 & 162.34 & $\mathrm{~S}_{\mathrm{AVG}}-2400$ & 1.00 \\
& 2500 & 203.75 & $\mathrm{~S}_{\mathrm{AVG}}-2500$ & 1.00 \\
& 2600 & 250.43 & $\mathrm{~S}_{\mathrm{AVG}}-2600$ & 1.00 \\
& 2700 & 302.15 & $\mathrm{~S}_{\mathrm{AVG}}-2700$ & 0.99 \\
\hline
\end{tabular}

In conclusion, taking a short position in a put option for 2006 and 2007 would be the best strategy for copper consumers. 


\subsection{FORWARD CONTRACTS AND METAL SWAPS}

The monthly average prices of copper on the LME will likely be greater than $\$ 3,000$ per tonne in 2006 and 2007, according to the simulation analysis in chapter 6. Therefore, it is not necessary to hedge and to implement a risk management strategy for copper producers during this period. By contrast, it is useful to implement a risk management strategy for a copper purchase for copper consumers because of the bullish trend in the copper market. Hence, it would be a profitable hedge for a copper consumer if he/she locked the purchasing price at $\$ 3,200$ or less in 2006 , and $\$ 3,400$ or less in 2007 .

The monthly SWAP prices that were offered to a copper producer on the day of data were $\$ 2,815$ in 2006 and $\$ 2,630$ in 2007. According to the simulation result in chapter 6, monthly average prices may be greater than $\$ 3,200$ in 2006 and $\$ 3,400$ in 2007; therefore, selling copper at the SWAP prices would not be an optimal risk management strategy to a copper producer on the day of data.

Finally, table (7.46) concludes evaluations of risk management strategies in 2006 and 2007 based on the day of data 22-February-2005. Columns 2-3 and 6-7 give the positions that could be profitable. The probabilities for risk management strategies to be profitable are given by table 4 and 8 and columns 5 and 9 show the total number of spreads for a specific position. 
Table 7.46: Evaluation of Risk Management Strategies in 2006-2007

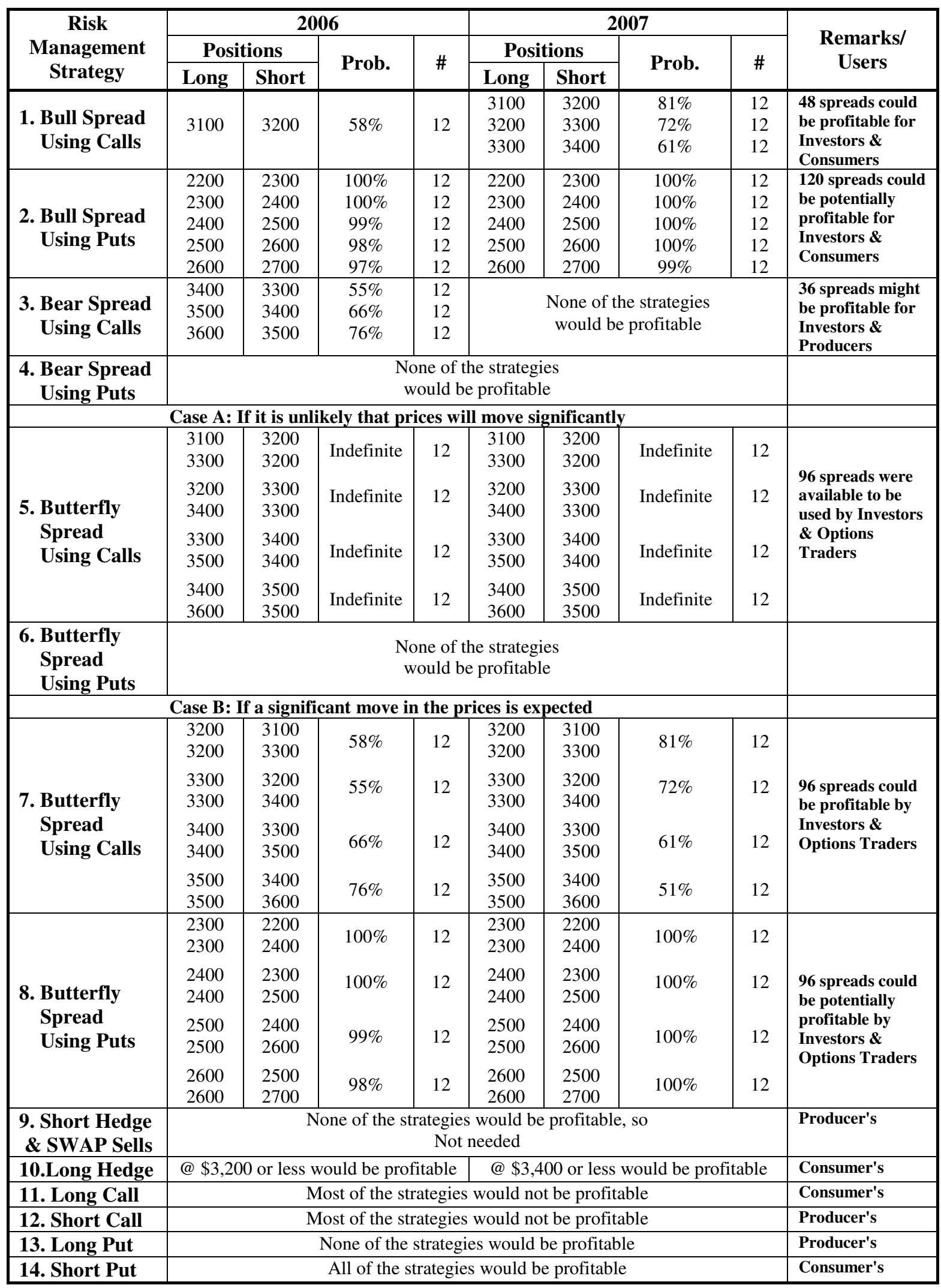




\section{CHAPTER VIII:}

\section{CONCLUSIONS AND RECOMMENDATIONS}

FOR FUTURE STUDIES

The main purpose of this research is the evaluation of risk management strategies for LME copper in the OTC market. The strategies include bull spreads, bear spreads, butterfly spreads involving both call and put options, average price call and put options (Asian), long and short hedges and metal SWAPS.

One of the sub-studies of this research is the valuation of Asian call and put options. This is because option traders, copper producers and consumers need to understand how theoretical options are valued and how much market observed option values are biased from their theoretically determined values. Asian options can be valued using the new MS Excel based software DerivaGem. This option pricing model is applied to 180 call and 180 put options for year of 2005, 2006 and 2007.

In order to evaluate risk management strategies, future price expectations are needed. Therefore, the following price prediction model for the LME copper is developed by a simultaneous econometric model using two-stage least squares (2SLS) regression.

$$
\left\{\begin{array}{l}
Q_{t}^{D}=e^{(-5.0485)} \cdot P_{(t-1)}^{(-0.1868)} \cdot G D P_{t}^{(1.7151)} \cdot e^{(0.0158) \cdot I P_{t}} \\
Q_{t}^{S}=e^{(-3.0785)} \cdot P_{(t-1)}^{(0.5960)} \cdot T_{t}^{(0.1408)} \cdot P_{O I L(t)}^{(-0.1559)} \cdot U S D I_{t}^{(1.2432)} \cdot \operatorname{LIBOR}_{(t-6)}^{(-0.0561)}
\end{array}\right.
$$

$\mathrm{P}_{(\mathrm{t}-1)}^{\mathrm{CU}}=$ Price of copper in $(\mathrm{t}-1)^{\text {th }}$ month $\quad \mathrm{e}=$ exponential constant 
$\mathrm{GDP}_{\mathrm{t}}=$ Gross domestic product in $\mathrm{t}^{\text {th }}$ month

$\mathrm{T}=$ Time variable to represent technological change

$\mathrm{USDI}_{\mathrm{t}}=\mathrm{U} . \mathrm{S}$. dollar index in $\mathrm{t}_{\mathrm{th}}$ month
$\mathrm{IP}_{\mathrm{t}}=$ Global IP growth in $\mathrm{t}^{\text {th }}$ month

$\mathrm{P}_{\mathrm{OIL}(\mathrm{t})}=$ Price of oil in $\mathrm{t}^{\text {th }}$ month

$\operatorname{LIBOR}_{(\mathrm{t}-6)}=\mathrm{LIBOR}-1 \mathrm{Y}$ in $(\mathrm{t}-6)^{\mathrm{th}}$ month

The econometric model specified and tested here is statistically significant and, in general, its results seem to be in accordance with underlying economic theories.

The relevant parameters obtained in the 2SLS regressions are significant by the t-test, and have the correct signs. The adjusted degrees of fit $\left(\bar{R}^{2}\right)$ are satisfactory, 0.64 and 0.91 for the structural equations of demand and supply respectively. Therefore, it is possible to explain the monthly copper prices on the LME by the following model:

$$
P_{(t-1)}^{C U}=e^{(-2.4455)} \cdot G D P_{t}^{(2.1178)} \cdot e^{(0.0194) \cdot I P_{t}} \cdot T_{t}^{(-0.1858)} \cdot P_{O I L(t)}^{(0.199)} \cdot U S D I_{t}^{(-1.4653)} \cdot \operatorname{LIBOR}_{t}^{(0.0537)}
$$

According to the results of Monte-Carlo Simulations on the LME copper prices, monthly average copper prices will likely be greater than $\$ 3,200$ in 2006 and $\$ 3,400$ in 2007. On the day of data (22-Feb-2005), 48 bull spreads involving call options, 120 bull spreads involving put options, 96 butterfly spreads involving call options and 96 butterfly spreads involving put options would be profitable and useful to investors, option traders, risk managers, producers and consumers for 2006 and 2007. Copper producers will benefit from high copper prices until the end of year 2007, but consumers will likely to face substantial price risks in the year of 2006 and 2007 due to the bullish trends in prices. On the day of data, taking a long position in the OTC market at $\$ 3,200$ or less in 2006 and $\$ 3,400$ or less in 2007 or writing a put option at any available strike price would be the most appropriate and optimal risk management strategy for copper 
consumers, according to the analysis result. Producers may not need to be hedged in these years because the expected prices have a bullish trend until the end of 2007.

In a bull market, consumers should implement risk management to insure against a rise in prices. In contrast, producers should implement risk management in a bear market to insure against a fall in prices. A bullish trend in the copper market is likely to continue until late 2007. Hence, copper consumers need to pay more attention for hedging and risk management until the market shifts to a bearish trend. However, at least 1.3 million tonnes of refined copper may be accumulated as stockpiled metal in China and this copper could be used to make good delivery against short positions, according to the Calyon's commodity research on 16 November 2005. In addition, analysts say that this can be a sign of bearish trend in the copper market and fall in copper prices. In this case, risk management will be very important to copper producers. As a result, when the company implements risk management, it is less risky than not being hedged and hedging may be better than no hedge in a potentially volatile copper market.

The following issues can be considered for future studies:

- In order for the model to be more accurate, the demand-side structural equation of the price prediction model may need to be improved, since omitted variables are likely.

- Risk management strategies can be extended by other OTC strategies such as producer MIN-MAX collars, structured put options, extra forward contracts and so forth.

- It will be an interesting and valuable research if the model and methodologies in this study are applied to other base metals market such as the aluminum market. 


\section{REFERENCES}

[1] AgOSTINI, Claudio [2002], "Estimating Market Power in the U.S. Copper Industry", University of Michigan, Ann Arbor, Working Paper, August 2002

[2] AHMED, Maqsood [2002], "LME Metals Weekly Update: 1, 8, 15, 22, 30 August \& 5, 12 September”, Credit Lyonnais Rouse Limited, London, United Kingdom; [2005], "LME Metals Weekly Update: 11 February and 01 March", Calyon Financial Metals Group, London, United Kingdom

[3] BLAKE, David [2000], “Financial Market Analysis”, $2^{\text {nd }}$ edition, John Wiley \& Sons Inc.

[4] BLOOMSBURY MINERALS ECONOMICS LIMITED [2003, 2004, 2005], “Copper Briefing Service", Monthly Bulletins

[5] CHANCE M. Don [2001], “An Introduction to Derivatives and Risk Management”, $5^{\text {th }}$ edition, Harcourt College Publishers

[6] CHR METALS LIMITED [Jan-2005], “CHR Economics: Global IP Watch”

[7] DAVIDSON, Russell and MACKINNON G. James [2004], "Econometric Theory and Methods”, Oxford University Press Inc.

[8] ENDERS, Walter [2004], “Applied Econometric Time Series”, Wiley series in probability and statistics, John Wiley \& Sons Inc.

[9] ERRERA, Steven and BROWN L. Stewart [2002], "Fundamentals of Trading Energy Futures and Options", $2^{\text {nd }}$ edition, PennWell Corporation

[10] GOSS B. A. [1972], “The Theory of Futures Trading”, Routledge and Kegan Paul

[11] GRANGER C. W. J. [1989], “Forecasting in Business and Economics”, $2^{\text {nd }}$ edition, Academic Press Inc.

[12] GREENE H. William [1990], “Econometric Analysis”, Macmillan Publishing Company, New York, Collier Macmillan Publishers, London

[13] HALL E. Robert and LIEBERMAN, Marc [2001], "Economics Principles and Applications", $2^{\text {nd }}$ edition, South-Western College Publishing

[14] HULL C. John [2002], “Fundamentals of Futures and Options Markets", $4^{\text {th }}$ edition, Pearson Education Inc.

[15] HULL C. John [2000], “Options, Futures, and Other Derivatives”, $4^{\text {th }}$ edition, Published by Prentice-Hall Inc. 
[16] HuSTED, Steven and MELVIN, Michael [2000], “International Economics", 5 edition, The Addison Wesley Series in Economics

[17] ICSG [2004], “Copper Bulletin”, Monthly Publication, Vol. 12, No. 4, April 2004

[18] JOHNSON, Dean [2004], “BA 4460: Derivatives Securities and Financial Engineering”, Class handout materials, School of Business and Economics, Michigan Technological University

[19] KOLB W. Robert [1993], “Financial Derivatives”, New York Institute of Finance

[20] LABYS C. Walter and GRANGER C. W. J. [1970], "Speculation, Hedging and Commodity Price Forecasts", Heath Lexington Books, D. C. Heath and Company, Lexington, Massachusetts

[21] LABYS C. Walter and POLLAK K. Peter [1984], "Commodity Models for Forecasting and Policy Analysis”, Nichols Publishing Company, New York

[22] MARTINOT, Natacha, LESOURD, Baptiste Jean and MORARD, Bernard [2000], “On the Information Content of Futures Prices: Application to LME non ferrous metal futures", Working Paper, June 2000

[23] MISHKIN S. Frederic and EAKINS G. Stanley [2000], "Financial Markets and Institutions", $3^{\text {rd }}$ edition, The Addison-Wesley Series in Finance

[24] OYEN B. Duane [1991], “Business Fluctuations \& Forecasting”, Dearborn Financial Publishing Inc.

[25] PHELPS DODGE CORP. [2002], “Technological issues in Morenci mine”, Presentation

[26] PINDYCK S. Robert and RUBINFELD L. Daniel [1981], "Econometric Models \& Economic Forecasts”, McGraw-Hill Book Company

[27] RITCHKEN, Peter [1996], “Derivative Markets: Theory, Strategy, and Applications", Harper Collins College Publishers

[28] ROBERTS C. Mark [1989], "Forecasting Mineral Consumption for Mine Project Evaluation”, Working paper, Multinational Conference On Mine Planning and Design, University of Kentucky, Lexington, Kentucky, May 23-26

[29] SANDAGDORJ, Bold [2004], "Application of Options Pricing in a Real World Situation”, Project, Advisor: Professor Dean L. Johnson, School of Business and Economics, Michigan Technological University, Houghton, Michigan, United States.

[30] SEIDEL D. Andrew and GINSBERG M. Philip [1983], "Commodities Trading: Foundations, Analysis, and Operations”, Published by Prentice-Hall Inc. 
[31] SIGMON, Hugh [1997], “Guide to Metal Trading”, Published by American Metal Market

[32] SLADE E. Margaret [1984], “ An Econometric Model of the U.S. Copper and Aluminum Industries: How Cost Changes Affect Substitution and Recycling”, Garland Publishing Inc., New York and London

[33] SOTOWICZ, Adam [2004], "LME Metal Price Model Tutorial”, Presentation, Bloomsbury Minerals Economics Ltd.

[34] StudEnMund A. H. [2001], "Using Econometrics: A Practical Guide”, 4 ${ }^{\text {th }}$ edition, Addison Wesley Longman Inc.

[35] TALEB, Nassim [1997], “Dynamic Hedging: Managing Vanilla and Exotic Options”, John Wiley \& Sons Inc.

[36] THE AMERICAN METAL MARKET CO., NEW YORK [1994, 1997, 2000, 2002], "Metal Statistics", The purchasing guide of the metal industries

[37] THE COMMODITY RESEARCH BUREAU [2004], "The CRB Commodity Yearbook”, John Wiley \& Sons Inc.

[38] THE LME [2001], "Introduction to Hedging with the LME and Introduction to LME Traded Options", Educational seminar, London, U.K.

[39] THE NYMEX [2003], “A Guide to Metals Hedging”, Study Material

[40] THE U.S. DEPARTMENT OF ENERGY [2004], "Kennecott Utah Copper Corporation: Facility Utilizes Energy Assessments to Identify \$930,000 in Potential Annual Savings”, Best Practices Plant-Wide Assessment Case Study, July 2004

[41] VERBEEK, Marno [2004], “A Guide to Modern Econometrics”, $2^{\text {nd }}$ edition, Erasmus University Rotterdam, John Wiley \& Sons Ltd.

[42] WilmotT, Paul, DeWYNNE, Jeff and HowISON, Sam [1993], "Option Pricing: Mathematical Models and Computation”, Published by Oxford Financial Press

[43] WILMOTT, Paul, HOWISON, Sam and DEWYNNE, Jeff [1995], "The Mathematics of Financial Derivatives: A Student Introduction”, Published by the Press Syndicate of the University of Cambridge 


\section{Web Pages:}

[1] Board of Governors of the Federal Reserve System http://federalreserve.gov/releases/H10/Summary/indexn_m.txt

[2] Economagic.com: Economic Time Series Page

http://www.economagician.com/em-cgi/data.exe/libor/day-us3m

[3] HSH Associates, Financial Publishers

http://www.hsh.com/indices/liborOOs.html

[4] Rates FX: Daily FOREX rates, Information about Currency Markets http://www.ratesfx.com/rates/rate-converter.html

[5] The International Monetary Fund:

http://www.imf.org/external/pubs/ft/weo/2002/02/data/index.htm\#changes

[6] The London Metal Exchange

http://www.lme.co.uk/

[7] The Metrod Corporation:

http://www.metrod.com/LMEAVG2004.htm

[8] The New York Mercantile Exchange

http://www.nymex.com/ 


\section{APPENDICES}

\section{Appendix 1: Implied Volatility (I.V.) Estimations}

Table A1-1: I.V. Estimation on 01.08.02

\begin{tabular}{|l|r|r|r|}
\hline $\begin{array}{l}\text { Date of Data } \\
\text { CU Price (\$/t) }\end{array}$ & $\begin{array}{r}\text { 01-Aug-02 } \\
\mathbf{1 4 7 8 . 0 0}\end{array}$ & \multicolumn{2}{|c|}{} \\
\hline \multicolumn{2}{|c|}{ T-Bill Discount Rates (\%) } & \multicolumn{2}{|c|}{ Risk-Free Interest Rate } \\
\hline 3-month & 1.68 & 0.017105 & $1.71 \%$ \\
6-month & 1.66 & 0.016972 & $1.70 \%$ \\
1-year & 1.75 & 0.018061 & $1.81 \%$ \\
\hline
\end{tabular}

\begin{tabular}{|c|c|c|c|c|c|c|c|c|}
\hline \multirow{3}{*}{$\begin{array}{c}\text { Strike } \\
\text { Price }\end{array}$} & \multicolumn{4}{|c|}{ 4Q-2002 CALL Options } & \multicolumn{4}{|c|}{ 2Q-2003 CALL Options } \\
\hline & \multirow{2}{*}{$\begin{array}{l}\text { Market } \\
\text { Value }\end{array}$} & \multicolumn{3}{|c|}{ Implied Volatilities } & \multirow{2}{*}{$\begin{array}{c}\text { Market } \\
\text { Value }\end{array}$} & \multicolumn{3}{|c|}{ Implied Volatilities } \\
\hline & & Oct- 02 & Nov-02 & Dec-02 & & Apr-03 & May-03 & Jun-03 \\
\hline 1525 & 43 & 112.10 & 85.32 & 68.16 & 94 & 59.66 & 54.34 & 50.43 \\
\hline 1575 & 26 & 100.60 & 76.73 & 61.46 & 72 & 56.10 & 51.16 & 47.54 \\
\hline 1600 & 20 & 96.01 & 73.37 & 58.87 & 63 & 54.72 & 49.95 & 46.44 \\
\hline Average & & 102.90 & 78.47 & 62.83 & & 56.83 & 51.82 & 48.14 \\
\hline
\end{tabular}

Table A1-2: I.V. Estimation on 08.08.02

\begin{tabular}{|l|r|r|r|}
\hline $\begin{array}{l}\text { Date of Data } \\
\text { CU Price (\$/t) }\end{array}$ & $\begin{array}{r}\mathbf{0 8 - A u g - 0 2} \\
\mathbf{1 4 6 7 . 0 0}\end{array}$ & \multicolumn{2}{|c|}{} \\
\hline \multicolumn{2}{|c|}{ T-Bill Discount Rates (\%) } & \multicolumn{2}{|c|}{ Risk-Free Interest Rate } \\
\hline 3-month & 1.62 & 0.016492 & $1.65 \%$ \\
6-month & 1.59 & 0.016250 & $1.63 \%$ \\
1-year & 1.68 & 0.017326 & $1.73 \%$ \\
\hline
\end{tabular}

\begin{tabular}{|c|c|c|c|c|c|c|c|c|}
\hline \multirow{3}{*}{$\begin{array}{c}\text { Strike } \\
\text { Price }\end{array}$} & \multicolumn{4}{|c|}{ 4Q-2002 CALL Options } & \multicolumn{4}{|c|}{ 2Q-2003 CALL Options } \\
\hline & \multirow{2}{*}{$\begin{array}{l}\text { Market } \\
\text { Value }\end{array}$} & \multicolumn{3}{|c|}{ Implied Volatilities } & \multirow{2}{*}{$\begin{array}{c}\text { Market } \\
\text { Value }\end{array}$} & \multicolumn{3}{|c|}{ Implied Volatilities } \\
\hline & & Oct-02 & Nov-02 & Dec-02 & & Apr-03 & May-03 & Jun-03 \\
\hline 1525 & 36 & 115.83 & 86.33 & 68.03 & 86 & 59.35 & 53.94 & 49.97 \\
\hline 1575 & 21 & 103.92 & 77.66 & 61.38 & 65 & 55.77 & 50.75 & 47.07 \\
\hline 1600 & 16 & 99.41 & 74.44 & 58.97 & 56 & 54.18 & 49.34 & 45.79 \\
\hline Average & & 106.39 & 79.48 & 62.79 & & 56.43 & 51.34 & 47.61 \\
\hline
\end{tabular}


Table A1-3: I.V. Estimation on 15.08.02

\begin{tabular}{|l|r|r|r|}
\hline $\begin{array}{l}\text { Date of Data } \\
\text { CU Price (\$/t) }\end{array}$ & $\begin{array}{r}\text { 15-Aug-02 } \\
\mathbf{1 4 7 4 . 0 0}\end{array}$ & \multicolumn{2}{c|}{} \\
\hline \multicolumn{2}{|c|}{ T-Bill Discount Rates (\%) } & \multicolumn{2}{c|}{ Risk-Free Interest Rate } \\
\hline 3-month & 1.62 & 0.0164919 & $1.65 \%$ \\
6-month & 1.63 & 0.0166626 & $1.67 \%$ \\
1-year & 1.78 & 0.0183763 & $1.84 \%$ \\
\hline
\end{tabular}

\begin{tabular}{|c|c|c|c|c|c|c|c|c|}
\hline \multirow{3}{*}{$\begin{array}{r}\text { Strike } \\
\text { Price }\end{array}$} & \multicolumn{4}{|c|}{ 4Q-2002 CALL Options } & \multicolumn{4}{|c|}{ 2Q-2003 CALL Options } \\
\hline & \multirow{2}{*}{$\begin{array}{l}\text { Market } \\
\text { Value }\end{array}$} & \multicolumn{3}{|c|}{ Implied Volatilities } & \multirow{2}{*}{$\begin{array}{l}\text { Market } \\
\text { Value }\end{array}$} & \multicolumn{3}{|c|}{ Implied Volatilities } \\
\hline & & Oct-02 & Nov-02 & Dec-02 & & Apr-03 & May-03 & Jun-03 \\
\hline 1525 & 36 & 125.07 & 90.90 & 70.45 & 87 & 60.14 & 54.43 & 50.28 \\
\hline 1575 & 20 & 110.67 & 80.58 & 62.60 & 66 & 56.58 & 51.28 & 47.44 \\
\hline 1600 & 15 & 105.53 & 77.00 & 59.96 & 57 & 55.00 & 49.89 & 46.18 \\
\hline Average & & 113.76 & 82.83 & 64.34 & & 57.24 & 51.87 & 47.97 \\
\hline
\end{tabular}

Table A1-4: I.V. Estimation on 22.08.02

\begin{tabular}{|l|r|r|r|}
\hline $\begin{array}{l}\text { Date of Data } \\
\text { CU Price (\$/t) }\end{array}$ & $\begin{array}{r}\text { 22-Aug-02 } \\
\mathbf{1 4 8 6 . 5 0}\end{array}$ & \multicolumn{2}{|c|}{} \\
\hline \multicolumn{2}{|c|}{ T-Bill Discount Rates (\%) } & \multicolumn{2}{|c|}{ Risk-Free Interest Rate } \\
\hline 3-month & 1.65 & 0.016799 & $1.68 \%$ \\
6-month & 1.68 & 0.017178 & $1.72 \%$ \\
1-year & 1.85 & 0.019113 & $1.91 \%$ \\
\hline
\end{tabular}

\begin{tabular}{|c|c|c|c|c|c|c|c|c|}
\hline \multirow{3}{*}{$\begin{array}{c}\text { Strike } \\
\text { Price }\end{array}$} & \multicolumn{4}{|c|}{ 4Q-2002 CALL Options } & \multicolumn{4}{|c|}{ 2Q-2003 CALL Options } \\
\hline & \multirow{2}{*}{$\begin{array}{l}\text { Market } \\
\text { Value }\end{array}$} & \multicolumn{3}{|c|}{ Implied Volatilities } & \multirow{2}{*}{$\begin{array}{c}\text { Market } \\
\text { Value }\end{array}$} & \multicolumn{3}{|c|}{ Implied Volatilities } \\
\hline & & Oct-02 & Nov-02 & Dec-02 & & Apr-03 & May-03 & Jun-03 \\
\hline 1525 & 37 & 136.54 & 96.30 & 73.18 & 90 & 61.05 & 55.01 & 50.66 \\
\hline 1575 & 21 & 121.29 & 85.71 & 65.32 & 68 & 57.20 & 51.62 & 47.60 \\
\hline 1600 & 15 & 114.11 & 80.74 & 61.63 & 59 & 55.68 & 50.29 & 46.42 \\
\hline Average & & 123.98 & 87.58 & 66.71 & & 57.98 & 52.31 & 48.23 \\
\hline
\end{tabular}

Table A1-5: I.V. Estimation on 30.08.02

\begin{tabular}{|l|r|r|r|}
\hline $\begin{array}{l}\text { Date of Data } \\
\text { CU Price (\$/t) }\end{array}$ & $\begin{array}{r}\text { 30-Aug-02 } \\
\mathbf{1 5 0 0 . 0 0}\end{array}$ & \multicolumn{2}{|c|}{} \\
\hline \multicolumn{2}{|c|}{ T-Bill Discount Rates (\%) } & \multicolumn{2}{l|}{ Risk-Free Interest Rate } \\
\hline 3-month & 1.69 & 0.0172075 & $1.72 \%$ \\
6-month & 1.67 & 0.0170749 & $1.71 \%$ \\
1-year & 1.74 & 0.0179559 & $1.80 \%$ \\
\hline
\end{tabular}

\begin{tabular}{|c|c|c|c|c|c|c|c|c|}
\hline \multirow{3}{*}{$\begin{array}{l}\text { Strike } \\
\text { Price }\end{array}$} & \multicolumn{4}{|c|}{ 4Q-2002 CALL Options } & \multicolumn{4}{|c|}{ 2Q-2003 CALL Options } \\
\hline & \multirow{2}{*}{$\begin{array}{l}\text { Market } \\
\text { Value }\end{array}$} & \multicolumn{3}{|c|}{ Implied Volatilities } & \multirow{2}{*}{$\begin{array}{c}\text { Market } \\
\text { Value }\end{array}$} & \multicolumn{3}{|c|}{ Implied Volatilities } \\
\hline & & Oct-02 & Nov-02 & Dec-02 & & Apr-03 & May-03 & Jun-03 \\
\hline 1525 & 40 & 155.61 & 105.58 & 78.37 & 97 & 63.72 & 57.29 & 52.57 \\
\hline 1575 & 22 & 136.39 & 92.59 & 68.84 & 74 & 59.66 & 53.72 & 49.36 \\
\hline 1600 & 16 & 128.69 & 87.49 & 65.17 & 64 & 57.83 & 52.12 & 47.92 \\
\hline Average & & 140.23 & 95.22 & 70.79 & & 60.40 & 54.38 & 49.95 \\
\hline
\end{tabular}


Table A1-6: I.V. Estimation on 21.02.05

\begin{tabular}{|l|r|r|r|}
\hline $\begin{array}{l}\text { Date of Data } \\
\text { CU Price (\$/t) }\end{array}$ & $\begin{array}{r}\text { 21-Feb-05 } \\
\mathbf{3 3 2 1 . 0 0}\end{array}$ & \multicolumn{2}{c|}{} \\
\hline \multicolumn{2}{|c|}{ T-Bill Discount Rates (\%) } & \multicolumn{2}{l|}{ Risk-Free Interest Rate } \\
\hline 6-month & 2.90 & 0.029618 & $2.96 \%$ \\
1-year & 3.09 & 0.031823 & $3.18 \%$ \\
2-year & 3.45 & 0.036245 & $3.62 \%$ \\
3-year & 3.62 & 0.038853 & $3.89 \%$ \\
\hline
\end{tabular}

\begin{tabular}{|c|c|c|c|c|c|c|c|}
\hline & \multicolumn{7}{|c|}{ 2005 CALL Options } \\
\cline { 2 - 8 } Strike & Market & \multicolumn{7}{|c|}{ Implied Volatilities } \\
\cline { 3 - 8 } Price & Value & July & August & September & October & November & December \\
\hline $\mathbf{3 1 0 0}$ & 192.56 & 68.95 & 56.55 & 47.43 & 41.20 & 35.95 & 31.45 \\
$\mathbf{3 2 0 0}$ & 154.87 & 66.82 & 55.21 & 46.69 & 40.88 & 35.99 & 31.81 \\
$\mathbf{3 3 0 0}$ & 123.27 & 65.04 & 54.11 & 46.09 & 40.62 & 36.02 & 32.09 \\
$\mathbf{3 4 0 0}$ & 97.17 & 63.54 & 53.20 & 45.60 & 40.41 & 36.05 & 32.33 \\
$\mathbf{3 5 0 0}$ & 75.89 & 62.28 & 52.43 & 45.19 & 40.24 & 36.08 & 32.52 \\
$\mathbf{3 6 0 0}$ & 58.77 & 61.21 & 51.80 & 44.86 & 40.11 & 36.12 & 32.69 \\
\hline Average & & 64.64 & 53.88 & 45.98 & 40.58 & 36.04 & 32.15 \\
\hline
\end{tabular}

\begin{tabular}{|c|c|c|c|c|c|c|c|}
\hline \multirow{3}{*}{$\begin{array}{c}\text { Strike } \\
\text { Price }\end{array}$} & Market & \multicolumn{7}{|c|}{ 2005 PUT Options } \\
\cline { 2 - 8 } & Value & July & August & September & October & November & December \\
\hline $\mathbf{2 2 0 0}$ & 12.46 & 43.30 & 42.79 & 41.83 & 40.82 & 39.85 & 38.72 \\
$\mathbf{2 3 0 0}$ & 20.52 & 42.12 & 42.05 & 41.37 & 40.54 & 39.71 & 38.68 \\
$\mathbf{2 4 0 0}$ & 30.19 & 39.96 & 40.43 & 40.11 & 39.51 & 38.86 & 37.98 \\
$\mathbf{2 5 0 0}$ & 45.75 & 38.46 & 39.55 & 39.60 & 39.23 & 38.76 & 38.01 \\
$\mathbf{2 6 0 0}$ & 63.55 & 35.85 & 37.73 & 38.27 & 38.19 & 37.96 & 37.39 \\
$\mathbf{2 7 0 0}$ & 89.82 & 33.73 & 36.58 & 37.67 & 37.91 & 37.91 & 37.52 \\
\hline Average & & 38.90 & 39.86 & 39.81 & 39.37 & 38.84 & 38.05 \\
\hline
\end{tabular}

\begin{tabular}{|c|c|c|c|c|c|c|c|}
\hline & \multicolumn{7}{|c|}{ 2006 CALL Options } \\
\cline { 3 - 8 } Strike & Market & \multicolumn{7}{|c|}{ Implied Volatilities } \\
\cline { 3 - 8 } Price & Value & January & February & March & April & May & June \\
\hline $\mathbf{3 1 0 0}$ & 151.14 & 22.10 & 19.64 & 17.14 & 15.20 & 13.39 & 11.62 \\
$\mathbf{3 2 0 0}$ & 125.34 & 24.43 & 22.16 & 19.87 & 18.13 & 16.54 & 15.03 \\
$\mathbf{3 3 0 0}$ & 103.42 & 26.21 & 24.04 & 21.86 & 20.21 & 18.71 & 17.30 \\
$\mathbf{3 4 0 0}$ & 84.95 & 27.63 & 25.53 & 23.42 & 21.82 & 20.37 & 19.01 \\
$\mathbf{3 5 0 0}$ & 69.47 & 28.79 & 26.74 & 24.68 & 23.12 & 21.70 & 20.37 \\
3600 & 56.59 & 29.77 & 27.76 & 25.73 & 24.19 & 22.80 & 21.48 \\
\hline Average & & 26.49 & 24.31 & 22.12 & 20.45 & 18.92 & 17.47 \\
\hline
\end{tabular}




\begin{tabular}{|c|c|c|c|c|c|c|c|}
\hline \multirow{3}{*}{$\begin{array}{c}\text { Strike } \\
\text { Price }\end{array}$} & \multicolumn{7}{|c|}{ 2006 CALL Options (continues) } \\
\cline { 2 - 8 } & Market & \multicolumn{7}{|c|}{ Implied Volatilities } \\
\cline { 3 - 8 } & Value & July & August & September & October & November & December \\
\hline $\mathbf{3 1 0 0}$ & 151.14 & 10.11 & 7.31 & 5.21 & - & - & - \\
$\mathbf{3 2 0 0}$ & 125.34 & 13.81 & 11.95 & 10.88 & 9.84 & 8.79 & 7.84 \\
$\mathbf{3 3 0 0}$ & 103.42 & 16.16 & 14.53 & 13.58 & 12.68 & 11.79 & 11.01 \\
$\mathbf{3 4 0 0}$ & 84.95 & 17.91 & 16.39 & 15.48 & 14.63 & 13.79 & 13.06 \\
$\mathbf{3 5 0 0}$ & 69.47 & 19.29 & 17.84 & 16.95 & 16.11 & 15.30 & 14.59 \\
$\mathbf{3 6 0 0}$ & 56.59 & 20.42 & 19.01 & 18.14 & 17.31 & 16.51 & 15.81 \\
\hline Average & & 16.28 & 14.51 & 13.37 & 14.11 & 13.24 & 12.46 \\
\hline
\end{tabular}

\begin{tabular}{|c|c|c|c|c|c|c|c|}
\hline \multirow{2}{*}{$\begin{array}{c}\text { Strike } \\
\text { Price }\end{array}$} & \multicolumn{7}{|c|}{ 2006 PUT Options } \\
\cline { 2 - 8 } & Market & \multicolumn{7}{|c|}{ Implied Volatilities } & April & May & June \\
\cline { 3 - 8 } & Value & January & February & March & Aprily \\
$\mathbf{2 2 0 0}$ & 58.87 & 56.26 & 54.83 & 53.27 & 52.00 & 50.79 & 49.59 \\
$\mathbf{2 3 0 0}$ & 81.54 & 57.07 & 55.67 & 54.14 & 52.89 & 51.68 & 50.49 \\
$\mathbf{2 4 0 0}$ & 104.74 & 56.93 & 55.59 & 54.12 & 52.91 & 51.74 & 50.58 \\
$\mathbf{2 5 0 0}$ & 137.61 & 57.94 & 56.63 & 55.18 & 53.98 & 52.83 & 51.68 \\
$\mathbf{2 6 0 0}$ & 170.62 & 58.03 & 56.78 & 55.39 & 54.25 & 53.13 & 52.01 \\
$\mathbf{2 7 0 0}$ & 214.64 & 59.25 & 58.04 & 56.67 & 55.54 & 54.43 & 53.32 \\
\hline Average & & 57.58 & 56.26 & 54.80 & 53.60 & 52.43 & 51.28 \\
\hline
\end{tabular}

\begin{tabular}{|c|c|c|c|c|c|c|c|}
\hline \multirow{2}{*}{$\begin{array}{c}\text { Strike } \\
\text { Price }\end{array}$} & Market & \multicolumn{7}{|c|}{ 2006 PUT Options (Continues) } \\
\cline { 2 - 7 } & Value & July & August & September & October & November & December \\
\cline { 2 - 8 } & 58.87 & 48.57 & 47.81 & 46.94 & 46.09 & 45.24 & 44.49 \\
$\mathbf{2 3 0 0}$ & 81.54 & 49.48 & 48.76 & 47.89 & 47.04 & 46.19 & 45.44 \\
$\mathbf{2 4 0 0}$ & 104.74 & 49.60 & 48.94 & 48.09 & 47.26 & 46.43 & 45.70 \\
$\mathbf{2 5 0 0}$ & 137.61 & 50.70 & 50.08 & 49.23 & 48.40 & 47.58 & 46.84 \\
$\mathbf{2 6 0 0}$ & 170.62 & 51.06 & 50.50 & 49.67 & 48.86 & 48.05 & 47.33 \\
$\mathbf{2 7 0 0}$ & 214.64 & 52.37 & 51.86 & 51.03 & 50.22 & 49.41 & 48.69 \\
\hline Average & & 50.30 & 49.66 & 48.81 & 47.98 & 47.15 & 46.42 \\
\hline
\end{tabular}

\begin{tabular}{|c|c|c|c|c|c|c|c|}
\hline \multirow{3}{*}{$\begin{array}{r}\text { Strike } \\
\text { Price }\end{array}$} & \multicolumn{7}{|c|}{2007 CALL Options } \\
\hline & \multirow{2}{*}{$\begin{array}{c}\text { Market } \\
\text { Value }\end{array}$} & \multicolumn{6}{|c|}{ Implied Volatilities } \\
\hline & & January & February & March & April & May & June \\
\hline 3100 & 117.13 & - & - & - & - & - & - \\
\hline 3200 & 98.12 & 2.99 & - & - & - & - & - \\
\hline 3300 & 81.95 & 8.14 & 7.53 & 6.88 & 6.30 & 5.66 & 5.10 \\
\hline 3400 & 68.24 & 10.65 & 10.11 & 9.52 & 9.02 & 8.49 & 8.04 \\
\hline 3500 & 56.68 & 12.50 & 11.94 & 11.40 & 10.91 & 10.40 & 9.97 \\
\hline 3600 & 46.96 & 13.96 & 13.44 & 12.87 & 12.39 & 11.88 & 11.46 \\
\hline Average & & 9.65 & 10.76 & 10.17 & 9.66 & 9.11 & 8.64 \\
\hline
\end{tabular}




\begin{tabular}{|c|c|c|c|c|c|c|c|}
\hline \multirow{3}{*}{$\begin{array}{r}\text { Strike } \\
\text { Price }\end{array}$} & \multicolumn{7}{|c|}{2007 CALL Options (continues) } \\
\hline & \multirow{2}{*}{$\begin{array}{c}\text { Market } \\
\text { Value }\end{array}$} & \multicolumn{6}{|c|}{ Implied Volatilities } \\
\hline & & July & August & September & October & November & December \\
\hline 3100 & 117.13 & - & - & - & - & - & - \\
\hline 3200 & 98.12 & - & - & - & - & - & - \\
\hline 3300 & 81.95 & 4.52 & 2.99 & 1.91 & - & - & - \\
\hline 3400 & 68.24 & 7.59 & 6.73 & 6.33 & 5.90 & 5.47 & 5.08 \\
\hline 3500 & 56.68 & 9.55 & 8.79 & 8.43 & 8.04 & 7.66 & 7.32 \\
\hline 3600 & 46.96 & 11.04 & 10.33 & 9.98 & 9.60 & 9.23 & 8.90 \\
\hline Average & & 8.18 & 7.21 & 6.66 & 7.85 & 7.45 & 7.10 \\
\hline
\end{tabular}

\begin{tabular}{|c|c|c|c|c|c|c|c|}
\hline \multirow{3}{*}{$\begin{array}{c}\text { Strike } \\
\text { Price }\end{array}$} & \multicolumn{7}{|c|}{ 2007 PUT Options } \\
\hline & \multirow{2}{*}{$\begin{array}{c}\text { Market } \\
\text { Value }\end{array}$} & \multicolumn{6}{|c|}{ Implied Volatilities } \\
\hline & & January & February & March & April & May & June \\
\hline 2200 & 105.27 & 52.99 & 52.21 & 51.37 & 50.65 & 49.87 & 49.23 \\
\hline 2300 & 137.48 & 54.29 & 53.51 & 52.66 & 51.94 & 51.15 & 50.50 \\
\hline 2400 & 168.40 & 54.73 & 53.95 & 53.12 & 52.40 & 51.62 & 50.98 \\
\hline 2500 & 210.81 & 56.20 & 55.42 & 54.58 & 53.85 & 53.07 & 52.42 \\
\hline 2600 & 258.49 & 57.75 & 56.96 & 56.11 & 55.37 & 54.58 & 53.92 \\
\hline 2700 & 311.20 & 59.36 & 58.56 & 57.70 & 56.95 & 56.16 & 55.49 \\
\hline Average & & 55.89 & 55.10 & 54.26 & 53.53 & 52.74 & 52.09 \\
\hline
\end{tabular}

\begin{tabular}{|c|c|c|c|c|c|c|c|}
\hline \multirow{2}{*}{$\begin{array}{c}\text { Strike } \\
\text { Price }\end{array}$} & Market & Implied Volatilities \\
\cline { 3 - 8 } & Value & July & August & September & October & November & December \\
\hline $\mathbf{2 2 0 0}$ & 105.27 & 48.59 & 48.21 & 47.67 & 47.11 & 46.55 & 46.05 \\
$\mathbf{2 3 0 0}$ & 137.48 & 49.86 & 49.50 & 48.96 & 48.39 & 47.83 & 47.32 \\
$\mathbf{2 4 0 0}$ & 168.40 & 50.34 & 50.02 & 49.49 & 48.92 & 48.37 & 47.87 \\
$\mathbf{2 5 0 0}$ & 210.81 & 51.78 & 51.48 & 50.94 & 50.37 & 49.81 & 49.30 \\
$\mathbf{2 6 0 0}$ & 258.49 & 53.27 & 53.00 & 52.46 & 51.88 & 51.32 & 50.81 \\
$\mathbf{2 7 0 0}$ & 311.20 & 54.83 & 54.59 & 54.04 & 53.46 & 52.88 & 52.37 \\
\hline Average & & 51.45 & 51.13 & 50.59 & 50.02 & 49.46 & 48.95 \\
\hline
\end{tabular}




\section{Appendix 2: The Results of Two-Stage Least Square Regression}

\section{Table A2-1: Summary Output of the Reduced Form Equation}

\begin{tabular}{lr}
\hline \multicolumn{2}{c}{ Regression Statistics } \\
\hline Multiple R & 0.882432794 \\
R Square & 0.778687637 \\
Adjusted R Square & 0.766936538 \\
Standard Error & 0.119314775 \\
Observations & 120 \\
\hline
\end{tabular}

\begin{tabular}{|c|c|c|c|c|c|c|c|c|}
\hline \multicolumn{9}{|l|}{ ANOVA } \\
\hline & $d f$ & $S S$ & $M S$ & $F$ & Significance $F$ & & & \\
\hline Regression & 6 & 5.66010515 & 0.943350858 & 66.26509066 & $1.01306 \mathrm{E}-34$ & & & \\
\hline Residual & 113 & 1.608669752 & 0.014236016 & & & & & \\
\hline \multirow[t]{2}{*}{ Total } & 119 & 7.268774902 & & & & & & \\
\hline & Coefficients & Standard Error & $t$ Stat & $P$-value & Lower 95\% & Upper 95\% & Lower $95.0 \%$ & Upper $95.0 \%$ \\
\hline Intercept & 6.145483052 & 6.289703456 & 0.977070397 & 0.330621428 & -6.315553503 & 18.60651961 & -6.315553503 & 18.60651961 \\
\hline LN[GDP] & 1.052093337 & 0.769288297 & 1.36761906 & 0.174145251 & -0.472005478 & 2.576192152 & -0.472005478 & 2.576192152 \\
\hline IP $\%$ & -0.00085807 & 0.006184824 & -0.13873839 & 0.889903832 & -0.013111323 & 0.011395178 & -0.013111323 & 0.011395178 \\
\hline $\mathrm{LN}[\mathrm{T}]$ & -0.12909579 & 0.028639815 & -4.50756368 & $1.60946 \mathrm{E}-05$ & -0.18583643 & -0.07235515 & -0.18583643 & -0.072355154 \\
\hline LN[P Oil] & 0.268063148 & 0.071967227 & 3.72479475 & 0.000306701 & 0.125483097 & 0.410643199 & 0.125483097 & 0.410643199 \\
\hline LN[USDI] & -1.60691204 & 0.227782929 & -7.05457622 & $1.47565 \mathrm{E}-10$ & -2.058191089 & -1.15563299 & -2.058191089 & -1.155632986 \\
\hline LN[Libor $1 Y(t-6)]$ & 0.094978381 & 0.045481106 & 2.088304098 & 0.039016835 & 0.004872109 & 0.185084653 & 0.004872109 & 0.185084653 \\
\hline
\end{tabular}

\section{Table A2-2: Summary Output of the Demand Equation}

\begin{tabular}{|c|c|c|c|c|c|c|c|c|}
\hline \multicolumn{2}{|c|}{ Regression Statistics } & & & & & & & \\
\hline $\begin{array}{l}\text { Multiple R } \\
\text {. }\end{array}$ & 0.803728263 & & & & & & & \\
\hline R Square & 0.645979121 & & & & & & & \\
\hline Adjusted R Square & 0.636823409 & & & & & & & \\
\hline Standard Error & 0.081998582 & & & & & & & \\
\hline Observations & 120 & & & & & & & \\
\hline \multicolumn{9}{|l|}{ ANOVA } \\
\hline & $d f$ & $S S$ & $M S$ & $F$ & Significance $F$ & & & \\
\hline Regression & 3 & 1.423181466 & 0.474393822 & 70.5547635 & $4.87246 \mathrm{E}-26$ & & & \\
\hline Residual & 116 & 0.779957024 & 0.006723767 & & & & & \\
\hline \multirow[t]{2}{*}{ Total } & 119 & 2.203138489 & & & & & & \\
\hline & Coefficients & Standard Error & $t$ Stat & $P$-value & Lower 95\% & Upper 95\% & Lower $95.0 \%$ & Upper $95.0 \%$ \\
\hline Intercept & -5.04850731 & 1.171494136 & -4.30946016 & $3.44789 \mathrm{E}-05$ & -7.368798978 & -2.72821564 & -7.368798978 & -2.728215637 \\
\hline $\mathrm{LN}[\mathrm{P}(\mathrm{t}-1)]$ & -0.18680493 & 0.043637288 & -4.28085569 & $3.85333 \mathrm{E}-05$ & -0.273234078 & -0.10037579 & -0.273234078 & -0.100375789 \\
\hline LN[GDP] & 1.71513405 & 0.137747297 & 12.4513082 & $4.07798 \mathrm{E}-23$ & 1.442308181 & 1.987959919 & 1.442308181 & 1.987959919 \\
\hline IP growth & 0.015779157 & 0.003954994 & 3.989678576 & 0.000116247 & 0.007945792 & 0.023612521 & 0.007945792 & 0.023612521 \\
\hline
\end{tabular}

\section{Table A2-3: Summary Output of the Supply Equation}

\begin{tabular}{|c|c|c|c|c|c|c|c|c|}
\hline \multicolumn{2}{|c|}{ Regression Statistics } & & & & & & & \\
\hline Multiple R & 0.954798344 & & & & & & & \\
\hline R Square & 0.911639878 & & & & & & & \\
\hline Adjusted R Square & 0.907764434 & & & & & & & \\
\hline Standard Error & 0.031964198 & & & & & & & \\
\hline Observations & 120 & & & & & & & \\
\hline \multicolumn{9}{|l|}{ ANOVA } \\
\hline & $d f$ & $S S$ & $M S$ & $F$ & Significance $F$ & & & \\
\hline Regression & 5 & 1.201709461 & 0.240341892 & 235.2349538 & $2.52191 \mathrm{E}-58$ & & & \\
\hline Residual & 114 & 0.116474934 & 0.00102171 & & & & & \\
\hline \multirow[t]{2}{*}{ Total } & 119 & 1.318184395 & & & & & & \\
\hline & Coefficients & Standard Error & $t$ Stat & $P$-value & Lower 95\% & Upper 95\% & Lower $95.0 \%$ & Upper $95.0 \%$ \\
\hline Intercept & -3.07851671 & 2.861267559 & -1.07592759 & 0.284231403 & -8.746665524 & 2.589632103 & -8.746665524 & 2.589632103 \\
\hline $\mathrm{LN}[\mathrm{P}(\mathrm{t}-1)]$ & 0.596017446 & 0.195177883 & 3.053714069 & 0.002813852 & 0.209371576 & 0.982663316 & 0.209371576 & 0.982663316 \\
\hline $\mathrm{LN}[\mathrm{T}]$ & 0.140832545 & 0.021434993 & 6.570216654 & $1.56535 \mathrm{E}-09$ & 0.098369992 & 0.183295099 & 0.098369992 & 0.183295099 \\
\hline LN[P Oil] & -0.15584734 & 0.065460607 & -2.38078057 & 0.018934024 & -0.285524297 & -0.02617039 & -0.285524297 & -0.026170387 \\
\hline LN[USDI] & 1.243162349 & 0.335793409 & 3.702164231 & 0.000330944 & 0.577958213 & 1.908366485 & 0.577958213 & 1.908366485 \\
\hline LN[Libor $1 Y(t-6)]$ & -0.05612547 & 0.012877799 & -4.35831206 & $2.88383 \mathrm{E}-05$ & -0.081636289 & -0.03061465 & -0.081636289 & -0.030614648 \\
\hline
\end{tabular}




\section{Appendix 3: The Results of Subsidiary Regressions}

\section{Table A3-1: Demand Equation: $\mathbf{L N}[\mathbf{P}(\mathrm{t}-1)]$ as dependent variable}

\begin{tabular}{|c|c|c|c|c|c|c|c|c|}
\hline \multicolumn{2}{|c|}{ Regression Statistics } & & & & & & & \\
\hline Multiple R & 0.613319472 & & & & & & & \\
\hline R Square & 0.376160775 & & & & & & & \\
\hline Adjusted R Square & 0.365496856 & & & & & & & \\
\hline Standard Error & 0.173722335 & & & & & & & \\
\hline Observations & 120 & & & & & & & \\
\hline \multicolumn{9}{|l|}{ ANOVA } \\
\hline & $d f$ & $S S$ & $M S$ & $F$ & Significance $F$ & & & \\
\hline Regression & 2 & 2.129109537 & 1.064554769 & 35.27416105 & $1.02743 \mathrm{E}-12$ & & & \\
\hline Residual & 117 & 3.530995613 & 0.03017945 & & & & & \\
\hline \multirow[t]{2}{*}{ Total } & 119 & 5.66010515 & & & & & & \\
\hline & Coefficients & Standard Error & $t$ Stat & $P$-value & Lower 95\% & Upper $95 \%$ & Lower $95.0 \%$ & Upper $95.0 \%$ \\
\hline Intercept & 10.993767 & 2.264278588 & 4.855306698 & $3.75434 \mathrm{E}-06$ & 6.509482054 & 15.4780519 & 6.509482054 & 15.47805194 \\
\hline LN[GDP] & -0.46039752 & 0.288711001 & -1.59466565 & 0.113484855 & -1.032174506 & 0.11137947 & -1.032174506 & 0.111379474 \\
\hline IP growth \% & 0.055587209 & 0.006618073 & 8.399304593 & $1.21654 \mathrm{E}-13$ & 0.042480463 & 0.06869396 & 0.042480463 & 0.068693955 \\
\hline
\end{tabular}

\section{Table A3-2: Demand Equation: IP growth \% as dependent variable}

\begin{tabular}{|c|c|c|c|c|c|c|c|c|}
\hline \multicolumn{2}{|c|}{ Regression Statistics } & & & & & & & \\
\hline Multiple R & 0.631431678 & & & & & & & \\
\hline R Square & 0.398705964 & & & & & & & \\
\hline Adjusted R Square & 0.388427434 & & & & & & & \\
\hline Standard Error & 1.916759087 & & & & & & & \\
\hline Observations & 120 & & & & & & & \\
\hline \multicolumn{9}{|l|}{ ANOVA } \\
\hline & $d f$ & $S S$ & $M S$ & $F$ & Significance $F$ & & & \\
\hline Regression & 2 & 285.0274972 & 142.5137486 & 38.7901717 & $1.19284 \mathrm{E}-13$ & & & \\
\hline Residual & 117 & 429.8539516 & 3.673965398 & & & & & \\
\hline \multirow[t]{2}{*}{ Total } & 119 & 714.8814488 & & & & & & \\
\hline & Coefficients & Standard Error & $t$ Stat & $P$-value & Lower 95\% & Upper 95\% & Lower $95.0 \%$ & Upper $95.0 \%$ \\
\hline Intercept & -112.700449 & 25.32468811 & -4.45022059 & $1.96873 \mathrm{E}-05$ & -162.8546646 & -62.5462325 & -162.8546646 & -62.54623249 \\
\hline $\mathrm{LN}[\mathrm{P}(\mathrm{t}-1)]$ & 6.767038031 & 0.805666464 & 8.399304593 & $1.21654 \mathrm{E}-13$ & 5.171457871 & 8.36261819 & 5.171457871 & 8.362618191 \\
\hline $\mathrm{LN}[\mathrm{GDP}]$ & 8.289155811 & 3.127391953 & 2.6505011 & 0.009149675 & 2.095520136 & 14.4827915 & 2.095520136 & 14.48279149 \\
\hline
\end{tabular}

\section{Table A3-3: Demand Equation: LN[GDP] as dependent variable}

\begin{tabular}{|c|c|c|c|c|c|c|c|c|}
\hline \multicolumn{2}{|c|}{ Regression Statistics } & & & & & & & \\
\hline Multiple R & 0.237997932 & & & & & & & \\
\hline R Square & 0.056643016 & & & & & & & \\
\hline Adjusted R Square & 0.040517255 & & & & & & & \\
\hline Standard Error & 0.055033904 & & & & & & & \\
\hline Observations & 120 & & & & & & & \\
\hline \multicolumn{9}{|l|}{ ANOVA } \\
\hline & $d f$ & $S S$ & $M S$ & $F$ & Significance $F$ & & & \\
\hline Regression & 2 & 0.021277314 & 0.010638657 & 3.512579516 & 0.033002744 & & & \\
\hline Residual & 117 & 0.354361485 & 0.003028731 & & & & & \\
\hline \multirow[t]{2}{*}{ Total } & 119 & 0.375638799 & & & & & & \\
\hline & Coefficients & Standard Error & $t$ Stat & $P$-value & Lower 95\% & Upper 95\% & Lower $95.0 \%$ & Upper $95.0 \%$ \\
\hline Intercept & 8.18320557 & 0.214125414 & 38.21688155 & $6.17365 \mathrm{E}-68$ & 7.759141423 & 8.60726972 & 7.759141423 & 8.607269717 \\
\hline IP growth \% & 0.006833385 & 0.002578148 & 2.6505011 & 0.009149675 & 0.001727497 & 0.01193927 & 0.001727497 & 0.011939273 \\
\hline $\mathrm{LN}[\mathrm{P}(\mathrm{t}-1)]$ & -0.04620429 & 0.028974281 & -1.59466565 & 0.113484855 & -0.103586334 & 0.01117775 & -0.103586334 & 0.011177753 \\
\hline
\end{tabular}




\section{Table A3-4: Supply Equation: $\mathrm{LN}[\mathrm{P}(\mathrm{t}-1)]$ as dependent variable}

\begin{tabular}{|c|c|c|c|c|c|c|c|c|}
\hline \multicolumn{2}{|c|}{ Regression Statistics } & & & & & & & \\
\hline $\begin{array}{l}\text { Multiple R } \\
\text {. }\end{array}$ & 0.997627931 & & & & & & & \\
\hline R Square & 0.995261489 & & & & & & & \\
\hline Adjusted R Square & 0.995096671 & & & & & & & \\
\hline Standard Error & 0.015271591 & & & & & & & \\
\hline Observations & 120 & & & & & & & \\
\hline \multicolumn{9}{|l|}{ ANOVA } \\
\hline & $d f$ & $S S$ & $M S$ & $F$ & Significance $F$ & & & \\
\hline Regression & 4 & 5.633284678 & 1.408321169 & 6038.556449 & $1.3018 \mathrm{E}-132$ & & & \\
\hline Residual & 115 & 0.026820472 & 0.000233221 & & & & & \\
\hline \multirow[t]{2}{*}{ Total } & 119 & 5.66010515 & & & & & & \\
\hline & Coefficients & Standard Error & $t$ Stat & $P$-value & Lower 95\% & Upper 95\% & Lower $95.0 \%$ & Upper $95.0 \%$ \\
\hline Intercept & 14.61466841 & 0.107178381 & 136.3583619 & $5.2446 \mathrm{E}-129$ & 14.40236867 & 14.82696815 & 14.40236867 & 14.82696815 \\
\hline $\mathrm{LN}[\mathrm{T}]$ & -0.10529938 & 0.002908908 & -36.1989403 & $1.06658 \mathrm{E}-64$ & -0.111061365 & -0.09953739 & -0.111061365 & -0.099537393 \\
\hline LN[P Oil] & 0.329079078 & 0.006038326 & 54.4983917 & $5.4463 \mathrm{E}-84$ & 0.317118316 & 0.34103984 & 0.317118316 & 0.34103984 \\
\hline LN[USDI] & -1.70019016 & 0.024547192 & -69.2621053 & $1.30995 \mathrm{E}-95$ & -1.748813425 & -1.6515669 & -1.748813425 & -1.651566904 \\
\hline LN[LIBOR 1Y(t-6)] & 0.050391111 & 0.003971716 & 12.6874897 & $1.33488 \mathrm{E}-23$ & 0.042523905 & 0.058258317 & 0.042523905 & 0.058258317 \\
\hline
\end{tabular}

\section{Table A3-5: Supply Equation: LN[T] as dependent variable}

\begin{tabular}{|c|c|c|c|c|c|c|c|c|}
\hline \multicolumn{2}{|c|}{ Regression Statistics } & & & & & & & \\
\hline Multiple R & 0.989279239 & & & & & & & \\
\hline R Square & 0.978673414 & & & & & & & \\
\hline Adjusted R Square & 0.977931619 & & & & & & & \\
\hline Standard Error & 0.139056583 & & & & & & & \\
\hline Observations & 120 & & & & & & & \\
\hline \multicolumn{9}{|l|}{ ANOVA } \\
\hline & $d f$ & $S S$ & $M S$ & $F$ & Significance $F$ & & & \\
\hline Regression & 4 & 102.0463297 & 25.51158243 & 1319.332597 & $4.68741 \mathrm{E}-95$ & & & \\
\hline Residual & 115 & 2.223724319 & 0.019336733 & & & & & \\
\hline \multirow[t]{2}{*}{ Total } & 119 & 104.270054 & & & & & & \\
\hline & Coefficients & Standard Error & t Stat & $P$-value & Lower 95\% & Upper 95\% & Lower $95.0 \%$ & Upper $95.0 \%$ \\
\hline Intercept & 125.4678539 & 4.24907326 & 29.52828681 & $1.6443 \mathrm{E}-55$ & 117.0512578 & 133.88445 & 117.0512578 & 133.88445 \\
\hline LN[P Oil] & 2.934786674 & 0.078762887 & 37.26103464 & $4.96837 \mathrm{E}-66$ & 2.778772558 & 3.09080079 & 2.778772558 & 3.09080079 \\
\hline LN[USDI] & -14.3309833 & 0.590032947 & -24.2884459 & $4.26953 \mathrm{E}-47$ & -15.49972499 & -13.1622416 & -15.49972499 & -13.16224165 \\
\hline LN[LIBOR 1Y(t-6)] & 0.37286533 & 0.043928083 & 8.488085703 & $8.41303 \mathrm{E}-14$ & 0.285852256 & 0.459878405 & 0.285852256 & 0.459878405 \\
\hline $\mathrm{LN}[\mathrm{P}(\mathrm{t}-1)]$ & -8.73052455 & 0.241181772 & -36.1989403 & $1.06658 \mathrm{E}-64$ & -9.208259211 & -8.2527899 & -9.208259211 & -8.252789898 \\
\hline
\end{tabular}

\section{Table A3-6: Supply Equation: LN $\left[P^{\mathrm{OIL}}\right]$ as dependent variable}

\begin{tabular}{|c|c|c|c|c|c|c|c|c|}
\hline \multicolumn{2}{|c|}{ Regression Statistics } & & & & & & & \\
\hline Multiple R & 0.990038533 & & & & & & & \\
\hline R Square & 0.980176297 & & & & & & & \\
\hline Adjusted R Square & 0.979486777 & & & & & & & \\
\hline Standard Error & 0.045533901 & & & & & & & \\
\hline Observations & 120 & & & & & & & \\
\hline \multicolumn{9}{|l|}{ ANOVA } \\
\hline & $d f$ & $S S$ & $M S$ & $F$ & Significance $F$ & & & \\
\hline Regression & 4 & 11.78927156 & 2.947317889 & 1421.534023 & $7.02644 \mathrm{E}-97$ & & & \\
\hline Residual & 115 & 0.238433658 & 0.002073336 & & & & & \\
\hline Total & 119 & 12.02770522 & & & & & & \\
\hline & Coefficients & Standard Error & $t$ Stat & $P$-value & Lower 95\% & Upper $95 \%$ & Lower $95.0 \%$ & Upper $95.0 \%$ \\
\hline Intercept & -42.4850181 & 0.958107401 & -44.3426468 & $3.7069 \mathrm{E}-74$ & -44.38284439 & -40.5871918 & -44.38284439 & -40.58719178 \\
\hline LN[USDI] & 4.936728188 & 0.129968478 & 37.9840424 & $6.42552 \mathrm{E}-67$ & 4.679285655 & 5.194170722 & 4.679285655 & 5.194170722 \\
\hline LN[LIBOR $1 Y(\mathrm{t}-6)]$ & -0.15309974 & 0.011519951 & -13.2899649 & $5.49552 \mathrm{E}-25$ & -0.175918542 & -0.13028093 & -0.175918542 & -0.130280934 \\
\hline $\mathrm{LN}[\mathrm{P}(\mathrm{t}-1)]$ & 2.925508851 & 0.053680646 & 54.4983917 & $5.4463 \mathrm{E}-84$ & 2.819177826 & 3.031839875 & 2.819177826 & 3.031839875 \\
\hline $\mathrm{LN}[\mathrm{T}]$ & 0.314675662 & 0.008445167 & 37.26103464 & 4.96837E-66 & 0.297947412 & 0.331403912 & 0.297947412 & 0.331403912 \\
\hline
\end{tabular}




\section{Table A3-7: Supply Equation: LN[USDI] as dependent variable}

\begin{tabular}{|c|c|c|c|c|c|c|c|c|}
\hline \multicolumn{2}{|c|}{ Regression Statistics } & & & & & & & \\
\hline Multiple R & 0.995047889 & & & & & & & \\
\hline R Square & 0.990120302 & & & & & & & \\
\hline Adjusted R Square & 0.989776661 & & & & & & & \\
\hline Standard Error & 0.00887652 & & & & & & & \\
\hline Observations & 120 & & & & & & & \\
\hline \multicolumn{9}{|l|}{ ANOVA } \\
\hline & $d f$ & $S S$ & $M S$ & $F$ & Significance $F$ & & & \\
\hline Regression & 4 & 0.90808728 & 0.22702182 & 2881.258046 & $2.8885 \mathrm{E}-114$ & & & \\
\hline Residual & 115 & 0.009061149 & $7.87926 \mathrm{E}-05$ & & & & & \\
\hline \multirow[t]{2}{*}{ Total } & 119 & 0.917148429 & & & & & & \\
\hline & Coefficients & Standard Error & $t$ Stat & $P$-value & Lower 95\% & Upper 95\% & Lower $95.0 \%$ & Upper $95.0 \%$ \\
\hline Intercept & 8.494864182 & 0.062086779 & 136.822433 & $3.5568 \mathrm{E}-129$ & 8.371882226 & 8.617846139 & 8.371882226 & 8.617846139 \\
\hline LN[LIBOR 1Y(t-6)] & 0.030981514 & 0.002107742 & 14.69891315 & $3.60576 \mathrm{E}-28$ & 0.026806483 & 0.035156545 & 0.026806483 & 0.035156545 \\
\hline $\mathrm{LN}[\mathrm{P}(\mathrm{t}-1)]$ & -0.57439992 & 0.008293134 & -69.2621053 & $1.30995 \mathrm{E}-95$ & -0.590827018 & -0.55797281 & -0.590827018 & -0.557972814 \\
\hline $\mathrm{LN}[\mathrm{T}]$ & -0.05839536 & 0.002404244 & -24.2884459 & $4.26953 \mathrm{E}-47$ & -0.063157704 & -0.05363301 & -0.063157704 & -0.053633014 \\
\hline LN[P Oil] & 0.187609549 & 0.004939168 & 37.9840424 & $6.42552 \mathrm{E}-67$ & 0.177826009 & 0.197393089 & 0.177826009 & 0.197393089 \\
\hline
\end{tabular}

\section{Table A3-8: Supply Equation: $\operatorname{LN}[\operatorname{LIBOR}(t-6)]$ as dependent variable}

\begin{tabular}{|c|c|c|c|c|c|c|c|c|}
\hline \multicolumn{2}{|c|}{ Regression Statistics } & & & & & & & \\
\hline Multiple R & 0.917551676 & & & & & & & \\
\hline R Square & 0.841901078 & & & & & & & \\
\hline Adjusted R Square & 0.836401985 & & & & & & & \\
\hline Standard Error & 0.231458554 & & & & & & & \\
\hline Observations & 120 & & & & & & & \\
\hline \multicolumn{9}{|l|}{ ANOVA } \\
\hline & $d f$ & $S S$ & $M S$ & $F$ & Significance $F$ & & & \\
\hline Regression & 4 & 32.80775164 & 8.20193791 & 153.098172 & $4.28767 \mathrm{E}-45$ & & & \\
\hline Residual & 115 & 6.160902166 & 0.053573062 & & & & & \\
\hline \multirow[t]{2}{*}{ Total } & 119 & 38.96865381 & & & & & & \\
\hline & Coefficients & Standard Error & $t$ Stat & $P$-value & Lower 95\% & Upper $95 \%$ & Lower $95.0 \%$ & Upper $95.0 \%$ \\
\hline Intercept & -173.704895 & 12.91889641 & -13.4457998 & $2.42012 \mathrm{E}-25$ & -199.2947414 & -148.115048 & -199.2947414 & -148.1150482 \\
\hline $\mathrm{LN}[\mathrm{P}(\mathrm{t}-1)]$ & 11.57528863 & 0.912338761 & 12.6874897 & $1.33488 \mathrm{E}-23$ & 9.768121191 & 13.38245607 & 9.768121191 & 13.38245607 \\
\hline $\mathrm{LN}[\mathrm{T}]$ & 1.033035796 & 0.121704214 & 8.488085703 & $8.41303 \mathrm{E}-14$ & 0.791963179 & 1.274108412 & 0.791963179 & 1.274108412 \\
\hline LN[P Oil] & -3.95595368 & 0.29766472 & -13.2899649 & $5.49552 \mathrm{E}-25$ & -4.545570184 & -3.36633718 & -4.545570184 & -3.366337177 \\
\hline LN[USDI] & 21.06510665 & 1.43310641 & 14.69891315 & $3.60576 \mathrm{E}-28$ & 18.22639876 & 23.90381454 & 18.22639876 & 23.90381454 \\
\hline
\end{tabular}




\section{Appendix 4: The Result Details of the Park Test}

Table A4-1: Demand Equation: $P_{(\mathrm{t}-1)}$ as $\mathrm{Z}$ variable

\begin{tabular}{|c|c|c|c|c|c|c|c|c|}
\hline \multicolumn{2}{|c|}{ Regression Statistics } & & & & & & & \\
\hline Multiple R & 0.079559404 & & & & & & & \\
\hline R Square & 0.006329699 & & & & & & & \\
\hline Adjusted R Square & -0.00209124 & & & & & & & \\
\hline Standard Error & 2.680448308 & & & & & & & \\
\hline Observations & 120 & & & & & & & \\
\hline \multicolumn{9}{|l|}{ ANOVA } \\
\hline & $d f$ & $S S$ & $M S$ & $F$ & Significance $F$ & & & \\
\hline Regression & 1 & 5.400545346 & 5.400545346 & 0.75166226 & 0.387710041 & & & \\
\hline Residual & 118 & 847.8067693 & 7.18480313 & & & & & \\
\hline \multirow[t]{2}{*}{ Total } & 119 & 853.2073147 & & & & & & \\
\hline & Coefficients & Standard Error & $t$ Stat & $P$-value & Lower 95\% & Upper 95\% & Lower $95.0 \%$ & Upper $95.0 \%$ \\
\hline Intercept & 8.3139705 & 17.41796095 & 0.477321687 & 0.63401597 & -26.1783338 & 42.8062748 & -26.1783338 & 42.8062748 \\
\hline $\mathrm{LN}\{\mathrm{LN}[\mathrm{P}(\mathrm{t}-1)]\}$ & -7.45364104 & 8.597201418 & -0.86698458 & 0.38771004 & -24.47844023 & 9.57115815 & -24.47844023 & 9.571158145 \\
\hline
\end{tabular}

Table A4-2: Demand Equation: GDP as $\mathrm{Z}$ variable

\begin{tabular}{|c|c|c|c|c|c|c|c|c|}
\hline \multicolumn{2}{|c|}{ Regression Statistics } & & & & & & & \\
\hline Multiple R & 0.013186753 & & & & & & & \\
\hline R Square & 0.00017389 & & & & & & & \\
\hline Adjusted R Square & -0.00829921 & & & & & & & \\
\hline Standard Error & 2.688738205 & & & & & & & \\
\hline Observations & 120 & & & & & & & \\
\hline \multicolumn{9}{|l|}{ ANOVA } \\
\hline & $d f$ & $S S$ & $M S$ & $F$ & Significance $F$ & & & \\
\hline Regression & 1 & 0.148364602 & 0.148364602 & 0.02052264 & 0.88633136 & & & \\
\hline Residual & 118 & 853.0589501 & 7.229313136 & & & & & \\
\hline \multirow[t]{2}{*}{ Total } & 119 & 853.2073147 & & & & & & \\
\hline & Coefficients & Standard Error & $t$ Stat & $P$-value & Lower 95\% & Upper 95\% & Lower $95.0 \%$ & Upper $95.0 \%$ \\
\hline Intercept & 3.429263628 & 71.30505812 & 0.048092852 & 0.96172355 & -137.7741625 & 144.63269 & -137.7741625 & 144.6326898 \\
\hline $\mathrm{LN}\{\mathrm{LN}[\mathrm{GDP}]\}$ & -4.95491707 & 34.58754691 & -0.14325726 & 0.88633136 & -73.44767558 & 63.5378414 & -73.44767558 & 63.53784143 \\
\hline
\end{tabular}

Table A4-3: Supply Equation: $P_{(t-1)}$ as $Z$ variable

\begin{tabular}{|c|c|c|c|c|c|c|c|c|}
\hline \multicolumn{2}{|c|}{ Regression Statistics } & & & & & & & \\
\hline Multiple R & 0.235168125 & & & & & & & \\
\hline R Square & 0.055304047 & & & & & & & \\
\hline Adjusted R Square & 0.047298149 & & & & & & & \\
\hline Standard Error & 2.374533244 & & & & & & & \\
\hline Observations & 120 & & & & & & & \\
\hline \multicolumn{9}{|l|}{ ANOVA } \\
\hline & $d f$ & $S S$ & $M S$ & $F$ & Significance $F$ & & & \\
\hline Regression & 1 & 38.94963348 & 38.9496335 & 6.90791312 & 0.009722629 & & & \\
\hline Residual & 118 & 665.3321587 & 5.63840812 & & & & & \\
\hline \multirow[t]{2}{*}{ Total } & 119 & 704.2817922 & & & & & & \\
\hline & Coefficients & Standard Error & $t$ Stat & $P$-value & Lower 95\% & Upper 95\% & Lower $95.0 \%$ & Upper $95.0 \%$ \\
\hline Intercept & -48.965805 & 15.43007832 & -3.17339964 & 0.0019214 & -79.52156052 & -18.4100494 & -79.52156052 & -18.41004945 \\
\hline $\mathrm{LN}\{\mathrm{LN}[\mathrm{P}(\mathrm{t}-1)]\}$ & 20.01710895 & 7.616017257 & 2.62829091 & 0.00972263 & 4.935321504 & 35.0988964 & 4.935321504 & 35.0988964 \\
\hline
\end{tabular}




\section{Table A4-4: Supply Equation: $P^{\mathrm{OIL}}$ as $\mathrm{Z}$ variable}

\begin{tabular}{lr}
\hline \multicolumn{2}{c}{ Regression Statistics } \\
\hline Multiple R & 0.087460725 \\
R Square & 0.007649378 \\
Adjusted R Square & -0.00076037 \\
Standard Error & 2.433687434 \\
Observations & 120 \\
\hline
\end{tabular}

ANOVA

\begin{tabular}{|c|c|c|c|c|c|c|c|c|}
\hline & $d f$ & $S S$ & $M S$ & $F$ & Significance $F$ & & & \\
\hline Regression & 1 & 5.387317976 & 5.38731798 & 0.90958441 & 0.34217434 & & & \\
\hline Residual & 118 & 698.8944743 & 5.92283453 & & & & & \\
\hline Total & 119 & 704.2817922 & & & & & & \\
\hline & Coefficients & Standard Error & $t$ Stat & $P$-value & Lower $95 \%$ & Upper $95 \%$ & Lower $95.0 \%$ & Upper $95.0 \%$ \\
\hline Intercept & -10.823743 & 2.535302418 & -4.2692118 & $3.9866 \mathrm{E}-05$ & -15.84433196 & -5.80315403 & -15.84433196 & -5.803154033 \\
\hline $\mathrm{LN}\{\mathrm{LN}[\mathrm{P}$ Oil $]\}$ & 2.098214192 & 2.200028541 & 0.95372135 & 0.34217434 & -2.258441225 & 6.454869608 & -2.258441225 & 6.454869608 \\
\hline
\end{tabular}

\section{Table A4-5: Supply Equation: USDI as Z variable}

\begin{tabular}{|c|c|c|c|c|c|c|c|c|}
\hline \multicolumn{2}{|c|}{ Regression Statistics } & & & & & & & \\
\hline Multiple R & 0.246695219 & & & & & & & \\
\hline R Square & 0.060858531 & & & & & & & \\
\hline Adjusted R Square & 0.052899705 & & & & & & & \\
\hline Standard Error & 2.367542237 & & & & & & & \\
\hline Observations & 120 & & & & & & & \\
\hline \multicolumn{9}{|l|}{ ANOVA } \\
\hline & $d f$ & $S S$ & $M S$ & $F$ & Significance $F$ & & & \\
\hline Regression & 1 & 42.86155537 & 42.8615554 & 7.64667189 & 0.006602815 & & & \\
\hline Residual & 118 & 661.4202369 & 5.60525624 & & & & & \\
\hline \multirow[t]{2}{*}{ Total } & 119 & 704.2817922 & & & & & & \\
\hline & Coefficients & Standard Error & $t$ Stat & $P$-value & Lower 95\% & Upper $95 \%$ & Lower $95.0 \%$ & Upper $95.0 \%$ \\
\hline Intercept & 38.73296868 & 17.05148914 & 2.27152997 & 0.02492712 & 4.966378324 & 72.49955904 & 4.966378324 & 72.49955904 \\
\hline $\mathrm{LN}\{\mathrm{LN}[\mathrm{USDI}]\}$ & -31.105033 & 11.24849548 & -2.76526163 & 0.00660282 & -53.38011616 & -8.82994979 & -53.38011616 & -8.829949785 \\
\hline
\end{tabular}

Table A4-6: Supply Equation: LIBOR 1Y(t-6) as Z variable

\begin{tabular}{|c|c|c|c|c|c|c|c|c|}
\hline \multicolumn{2}{|c|}{ Regression Statistics } & & & & & & & \\
\hline Multiple R & 0.119480219 & & & & & & & \\
\hline R Square & 0.014275523 & & & & & & & \\
\hline Adjusted R Square & 0.005921925 & & & & & & & \\
\hline Standard Error & 2.425548691 & & & & & & & \\
\hline Observations & 120 & & & & & & & \\
\hline \multicolumn{9}{|l|}{ ANOVA } \\
\hline & $d f$ & $S S$ & $M S$ & $F$ & Significance $F$ & & & \\
\hline Regression & 1 & 10.05399066 & 10.0539907 & 1.70890721 & 0.19366878 & & & \\
\hline Residual & 118 & 694.2278016 & 5.88328645 & & & & & \\
\hline Total & 119 & 704.2817922 & & & & & & \\
\hline & Coefficients & Standard Error & $t$ Stat & $P$-value & Lower 95\% & Upper 95\% & Lower $95.0 \%$ & Upper $95.0 \%$ \\
\hline Intercept & -8.31640089 & 0.233932946 & -35.5503618 & $6.8924 \mathrm{E}-65$ & -8.779651806 & -7.85314997 & -8.779651806 & -7.853149973 \\
\hline $\mathrm{LN}\{\mathrm{LN}[$ Libor $1 \mathrm{Y}(\mathrm{t}-6)]\}$ & -0.45940631 & 0.351429094 & -1.30725178 & 0.19366878 & -1.155331582 & 0.236518966 & -1.155331582 & 0.236518966 \\
\hline
\end{tabular}




\section{Appendix 5: The Result Details of the White Test}

\begin{tabular}{|c|c|c|c|c|c|c|c|c|}
\hline \multicolumn{2}{|c|}{ Regression Statistics } & & & & & & & \\
\hline Multiple R & 0.224551588 & & & & & & & \\
\hline R Square & 0.050423416 & & & & & & & \\
\hline Adjusted R Square & -0.02726921 & & & & & & & \\
\hline Standard Error & 0.010681671 & & & & & & & \\
\hline Observations & 120 & & & & & & & \\
\hline \multicolumn{9}{|l|}{ ANOVA } \\
\hline & $d f$ & $S S$ & $M S$ & $F$ & Significance $F$ & & & \\
\hline$\overline{\text { Regression }}$ & 9 & 0.000666459 & $7.4051 \mathrm{E}-05$ & 0.64901157 & 0.752841279 & & & \\
\hline Residual & 110 & 0.01255079 & 0.000114098 & & & & & \\
\hline \multirow[t]{2}{*}{ Total } & 119 & 0.013217249 & & & & & & \\
\hline & Coefficients & Standard Error & $t$ Stat & $P$-value & Lower 95\% & Upper 95\% & Lower $95.0 \%$ & Upper $95.0 \%$ \\
\hline Intercept & 35.14957703 & 40.80201687 & 0.861466656 & 0.39085508 & -45.71044097 & 116.009595 & -45.71044097 & 116.009595 \\
\hline $\mathrm{LN}[\mathrm{P}(\mathrm{t}-1)]$ & -0.27937567 & 1.374582703 & -0.20324399 & 0.83931983 & -3.003475864 & 2.44472453 & -3.003475864 & 2.444724527 \\
\hline LN[GDP] & -8.61534881 & 10.51945307 & -0.81899209 & 0.41456176 & -29.46243505 & 12.2317374 & -29.46243505 & 12.23173743 \\
\hline IP $\%$ & 0.009115653 & 0.140925821 & 0.06468405 & 0.94854297 & -0.270166239 & 0.28839754 & -0.270166239 & 0.288397545 \\
\hline $\mathrm{LN}[\mathrm{P}(\mathrm{t}-1)]^{2}$ & -0.00907891 & 0.043896933 & -0.20682329 & 0.83653023 & -0.096072323 & 0.07791451 & -0.096072323 & 0.077914507 \\
\hline $\mathrm{LN}[\mathrm{GDP}]^{2}$ & 0.518167671 & 0.689969051 & 0.751001324 & 0.45425547 & -0.849188998 & 1.88552434 & -0.849188998 & 1.885524341 \\
\hline$(\mathrm{IP} \%)^{2}$ & 0.000253163 & 0.000278148 & 0.91017365 & 0.36472103 & -0.000298061 & 0.00080439 & -0.000298061 & 0.000804388 \\
\hline $\mathrm{LN}[\mathrm{P}(\mathrm{t}-1)] * \mathrm{LN}[\mathrm{GDP}]$ & 0.053739956 & 0.138693189 & 0.387473651 & 0.6991543 & -0.221117382 & 0.32859729 & -0.221117382 & 0.328597294 \\
\hline $\mathrm{LN}[\mathrm{P}(\mathrm{t}-1)] * \mathrm{IP} \%$ & -0.00274255 & 0.006093909 & -0.4500471 & 0.65356215 & -0.014819242 & 0.00933415 & -0.014819242 & 0.00933415 \\
\hline LN[GDP]*IP \% & 0.001262394 & 0.015237565 & 0.082847519 & 0.93412336 & -0.028934882 & 0.03145967 & -0.028934882 & 0.031459671 \\
\hline
\end{tabular}




\section{Appendix 6: The Results of Two-Stage Least Square Regression}

\section{(Including $\mathrm{P}_{(\mathrm{t}-1)}^{\mathrm{AL}}$ as an exogenous variable in demand equation)}

\section{Table A6-1: Summary Output of the Demand Equation}

\begin{tabular}{|c|c|c|c|c|c|c|c|c|}
\hline \multicolumn{2}{|c|}{ Regression Statistics } & & & & & & & \\
\hline Multiple R & 0.813278905 & & & & & & & \\
\hline R Square & 0.661422578 & & & & & & & \\
\hline Adjusted R Square & 0.649645972 & & & & & & & \\
\hline Standard Error & 0.080538025 & & & & & & & \\
\hline Observations & 120 & & & & & & & \\
\hline \multicolumn{9}{|l|}{ ANOVA } \\
\hline & $d f$ & $S S$ & $M S$ & $F$ & Significance $F$ & & & \\
\hline Regression & 4 & 1.457205539 & 0.364301385 & 56.16410862 & $3.52171 \mathrm{E}-26$ & & & \\
\hline Residual & 115 & 0.74593295 & 0.006486373 & & & & & \\
\hline \multirow[t]{2}{*}{ Total } & 119 & 2.203138489 & & & & & & \\
\hline & Coefficients & Standard Error & $t$ Stat & $P$-value & Lower 95\% & Upper 95\% & Lower $95.0 \%$ & Upper $95.0 \%$ \\
\hline Intercept & -7.13123812 & 1.383192377 & -5.15563723 & $1.06284 \mathrm{E}-06$ & -9.871075924 & -4.39140032 & -9.871075924 & -4.39140032 \\
\hline $\mathrm{LN}[\mathrm{P}(\mathrm{t}-1)]$ & -0.34611209 & 0.085954327 & -4.02669773 & 0.000101781 & -0.516371065 & -0.17585312 & -0.516371065 & -0.175853119 \\
\hline $\mathrm{LN}[\mathrm{GDP}]$ & 1.743776284 & 0.135261572 & 12.89188241 & $4.50812 \mathrm{E}-24$ & 1.47584915 & 2.011703417 & 1.47584915 & 2.011703417 \\
\hline IP growth & 0.013645737 & 0.003762323 & 3.626944687 & 0.00042895 & 0.0061933 & 0.021098174 & 0.0061933 & 0.021098174 \\
\hline $\mathrm{LN}[\mathrm{P}(\mathrm{Al})(\mathrm{t}-1)]$ & 0.420229223 & 0.179859863 & 2.336425792 & 0.021200505 & 0.063961462 & 0.776496984 & 0.063961462 & 0.776496984 \\
\hline
\end{tabular}

$\overline{\operatorname{Ln}\left[Q_{t}^{D}\right]}=-7.1312-0.3461 \cdot \operatorname{Ln}\left[P_{(t-1)}\right]+1.7438 \cdot \operatorname{Ln}\left[G D P_{t}\right]+0.0137 \cdot I P_{t}+0.4202 \cdot \operatorname{Ln}\left[P_{(t-1)}^{A L}\right]$

Standard error: $\quad(0.0860) \quad(0.1353) \quad(0.0038)$

$(0.1799)$

T-statistics:

$$
\mathrm{R}^{2}=0.66, \quad \bar{R}^{2}=0.65, \quad \mathrm{~F}=56.16, \quad \mathrm{n}=120
$$

Table A6-2: Summary Output of the Supply Equation

\begin{tabular}{|c|c|c|c|c|c|c|c|c|}
\hline \multicolumn{2}{|c|}{ Regression Statistics } & & & & & & & \\
\hline Multiple R & 0.951623077 & & & & & & & \\
\hline R Square & 0.905586481 & & & & & & & \\
\hline Adjusted R Square & 0.901445537 & & & & & & & \\
\hline Standard Error & 0.033040967 & & & & & & & \\
\hline Observations & 120 & & & & & & & \\
\hline \multicolumn{9}{|l|}{ ANOVA } \\
\hline & $d f$ & $S S$ & $M S$ & $F$ & Significance $F$ & & & \\
\hline Regression & 5 & 1.193729967 & 0.238745993 & 218.6908388 & $1.09097 \mathrm{E}-56$ & & & \\
\hline Residual & 114 & 0.124454428 & 0.001091706 & & & & & \\
\hline \multirow[t]{2}{*}{ Total } & 119 & 1.318184395 & & & & & & \\
\hline & Coefficients & Standard Error & $t$ Stat & $P$-value & Lower 95\% & Upper $95 \%$ & Lower $95.0 \%$ & Upper $95.0 \%$ \\
\hline Intercept & 5.023697921 & 0.561051783 & 8.954071754 & $7.5153 \mathrm{E}-15$ & 3.912258696 & 6.135137146 & 3.912258696 & 6.135137146 \\
\hline $\mathrm{LN}[\mathrm{P}(\mathrm{t}-1)]$ & 0.041628225 & 0.034957285 & 1.190831171 & 0.236193512 & -0.027621886 & 0.110878336 & -0.027621886 & 0.110878336 \\
\hline $\mathrm{LN}[\mathrm{T}]$ & 0.082455705 & 0.007291014 & 11.30922308 & $2.42173 \mathrm{E}-20$ & 0.068012262 & 0.096899147 & 0.068012262 & 0.096899147 \\
\hline LN[P Oil] & 0.026590552 & 0.017407195 & 1.527560952 & 0.129391458 & -0.007892967 & 0.06107407 & -0.007892967 & 0.06107407 \\
\hline LN[USDI] & 0.300595248 & 0.079705697 & 3.77131449 & 0.00025911 & 0.142698882 & 0.458491614 & 0.142698882 & 0.458491614 \\
\hline LN[LIBOR 1Y(t-6)] & -0.02818918 & 0.008771733 & -3.21363858 & 0.001704615 & -0.045565914 & -0.01081244 & -0.045565914 & -0.010812445 \\
\hline
\end{tabular}




$$
\left.\overline{\operatorname{Ln}\left[Q_{t}^{S}\right.}\right]=5.0237+0.0416 \cdot \operatorname{Ln}\left[P_{(t-1)}\right]+0.0825 \cdot \operatorname{Ln}\left[T_{t}\right]+0.0266 \cdot \operatorname{Ln}\left[P_{t}^{O I L}\right]+
$$

Standard error: $\quad(0.0350) \quad(0.0073) \quad(0.0174)$

$\begin{array}{lll}\text { T-statistics: } & 1.1908 & 11.3092\end{array}$

$$
+0.3006 \cdot \operatorname{Ln}\left[U S D I_{t}\right]-0.0282 \cdot \operatorname{Ln}\left[\operatorname{LIBOR}(1 Y)_{(t-6)}\right]
$$

Standard error: $\quad(0.0797) \quad(0.0088)$

T-statistics: $\quad 3.7713 \quad-3.2136$

$$
\mathrm{R}^{2}=0.91, \quad \bar{R}^{2}=0.90, \quad \mathrm{~F}=218.69, \quad \mathrm{n}=120
$$




\section{Appendix 7: The Results of Two-Stage Least Square Regression}

\section{(Interest rate variable is lagged by 12 months)}

\section{Table A7-1: Summary Output of the Demand Equation}

\begin{tabular}{|c|c|c|c|c|c|c|c|c|}
\hline \multicolumn{2}{|c|}{ Regression Statistics } & & & & & & & \\
\hline Multiple R & 0.803904174 & & & & & & & \\
\hline R Square & 0.646261921 & & & & & & & \\
\hline Adjusted R Square & 0.637113522 & & & & & & & \\
\hline Standard Error & 0.081965824 & & & & & & & \\
\hline Observations & 120 & & & & & & & \\
\hline \multicolumn{9}{|l|}{ ANOVA } \\
\hline & $d f$ & $S S$ & $M S$ & $F$ & Significance $F$ & & & \\
\hline Regression & 3 & 1.423804512 & 0.474601504 & 70.64208173 & $4.65277 \mathrm{E}-26$ & & & \\
\hline Residual & 116 & 0.779333977 & 0.006718396 & & & & & \\
\hline \multirow[t]{2}{*}{ Total } & 119 & 2.203138489 & & & & & & \\
\hline & Coefficients & Standard Error & $t$ Stat & P-value & Lower 95\% & Upper 95\% & Lower $95.0 \%$ & Upper $95.0 \%$ \\
\hline Intercept & -5.02804808 & 1.172487445 & -4.28835984 & $3.74274 \mathrm{E}-05$ & -7.350307122 & -2.70578903 & -7.350307122 & -2.705789032 \\
\hline $\mathrm{LN}[\mathrm{P}(\mathrm{t}-1)]$ & -0.18866592 & 0.043943445 & -4.29338021 & $3.67046 \mathrm{E}-05$ & -0.275701445 & -0.10163039 & -0.275701445 & -0.101630391 \\
\hline LN[GDP] & 1.714277257 & 0.137714076 & 12.44809033 & $4.14896 \mathrm{E}-23$ & 1.441517186 & 1.987037328 & 1.441517186 & 1.987037328 \\
\hline IP growth & 0.015882604 & 0.003964472 & 4.006234287 & 0.000109323 & 0.008030468 & 0.02373474 & 0.008030468 & 0.02373474 \\
\hline
\end{tabular}

Table A7-2: Summary Output of the Supply Equation

\begin{tabular}{|c|c|c|c|c|c|c|c|c|}
\hline \multicolumn{2}{|c|}{ Regression Statistics } & & & & & & & \\
\hline Multiple R & 0.955703493 & & & & & & & \\
\hline R Square & 0.913369167 & & & & & & & \\
\hline Adjusted R Square & 0.909569569 & & & & & & & \\
\hline Standard Error & 0.031649868 & & & & & & & \\
\hline Observations & 120 & & & & & & & \\
\hline \multicolumn{9}{|l|}{ ANOVA } \\
\hline & $d f$ & $S S$ & $M S$ & $F$ & Significance $F$ & & & \\
\hline Regression & 5 & 1.203988982 & 0.240797796 & 240.38574 & $8.19717 \mathrm{E}-59$ & & & \\
\hline Residual & 114 & 0.114195413 & 0.001001714 & & & & & \\
\hline \multirow[t]{2}{*}{ Total } & 119 & 1.318184395 & & & & & & \\
\hline & Coefficients & Standard Error & $t$ Stat & $P$-value & Lower 95\% & Upper 95\% & Lower $95.0 \%$ & Upper $95.0 \%$ \\
\hline Intercept & 4.11618332 & 5.608534466 & 0.733914242 & 0.46450775 & -6.994279901 & 15.22664654 & -6.994279901 & 15.22664654 \\
\hline $\mathrm{LN}[\mathrm{P}(\mathrm{t}-1)]$ & 0.081144383 & 0.389228383 & 0.208474989 & 0.83523013 & -0.689914021 & 0.852202787 & -0.689914021 & 0.852202787 \\
\hline $\mathrm{LN}[\mathrm{T}]$ & 0.082627036 & 0.046717605 & 1.768648824 & 0.07962711 & -0.009920175 & 0.175174248 & -0.009920175 & 0.175174248 \\
\hline LN[P Oil] & -0.00115352 & 0.126813878 & -0.00909613 & 0.99275834 & -0.252370823 & 0.250063792 & -0.252370823 & 0.250063792 \\
\hline LN[USDI] & 0.46050006 & 0.63686041 & 0.723078484 & 0.47111261 & -0.801115466 & 1.722115585 & -0.801115466 & 1.722115585 \\
\hline LN[Libor 1Y (t-12)] & -0.04954203 & 0.014028317 & -3.53157302 & 0.00059728 & -0.077332015 & -0.02175204 & -0.077332015 & -0.021752039 \\
\hline
\end{tabular}


Appendix 8: Fluctuations of the LME CU Prices and Major Influential Economic and Market Events (Source: AIG)

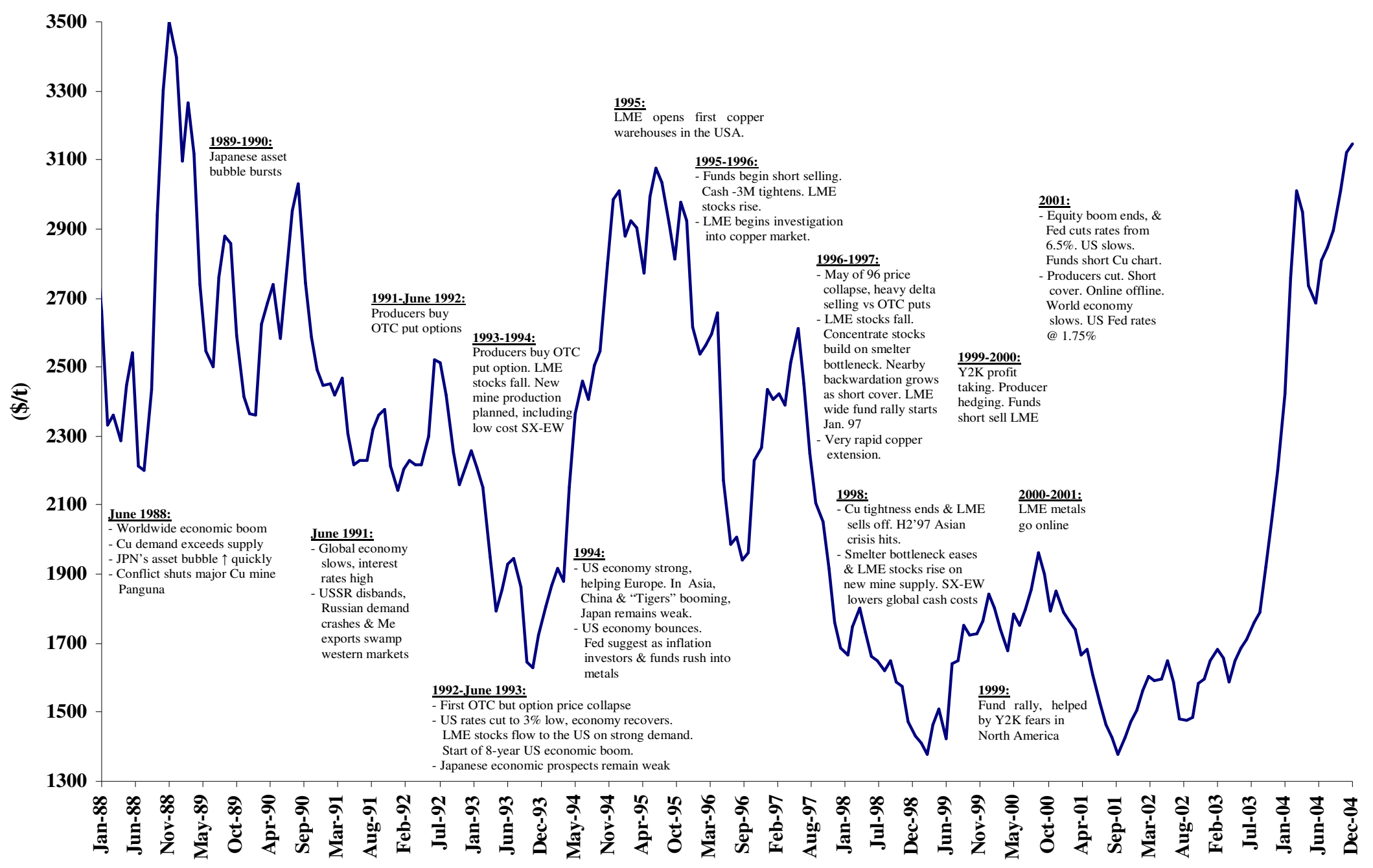

